\title{
TRANSJUGULAR INTRAHEPATIC PORTOSYSTEMIC STENT-SHUNT
}

by

\author{
Dr Rajiv Jalan
}

Presented for the degree of Doctor of Medicine at the University of Edinburgh

2000 


\section{Declaration of Originality}

I declare that the work presented herein and the composition of this thesis is my own. 


\section{ACKNOWLEDGMENTS}

My special thanks go to my supervisors, $\operatorname{Dr}$ Peter $C$ Hayes and $D r$ Doris $N$ Redhead for their advice, unwavering support, technical help and considerable on-going interest, encouragement and guidance for the performance of these studies. I would like to thank $\operatorname{Dr} N D C$ Finlayson and $D r$ AJ MacGilchrist for continued support and unlimited access to their patients. I would like to thank $\operatorname{Dr} K J$ Simpson and $\operatorname{Dr} N$ Chalmers who performed the early pilot studies which were the basis of this thesis.

I want to acknowledge the help of my collaborators without whom this work would be incomplete. The specific people who helped with the various parts of different studies are acknowledged below. Dr DN Redhead was instrumental in the performance of all the Transjugular intrahepatic portosystemic stent-shunts and the placement of catheters for the various studies. Dr EH Forrest provided considerable help for the completion of the studies requiring reverse thermodilution and also with the follow up for the secondary prophylaxis study. $\operatorname{Dr}$ AJ Stanley also helped with both patient recruitment and follow up patients in the secondary prophylaxis study. $\operatorname{Dr} R$ O'Carrol and M/s R Gooday were instrumental in the performance of the neuropsychological function tests. DrRA Elton provided the statistical support for the development of the prognostic models. $\operatorname{Dr} D J$ Harrison provided access to the post-mortem livers and also helped with immunohistochemical staining for defining the pathological changes after TIPSS. Mr TG John and 
Professor OJ Garden provided access to the surgical database which allowed successful comparison of TIPSS with transection for uncontrolled variceal haemorrhage. $\operatorname{Dr} P L$ Allan and $\mathrm{Dr}$ JM Ferguson helped with Doppler examinations of the shunts and therefore for the successful completion of the study comparing portography with Doppler examinations for the follow up of shunt function. Dr J Forbes performed most of the cost analysis for TIPSS and variceal band ligation in the secondary prophylaxis of variceal haemorrhage in patients with cirrhosis. Mr JJ Morton, Dr H Thomas and Dr N Henderson helped with the assay for the neurohumoral factors. I am grateful for Dr AD Cumming, Professor M Lee and Professor IAD Bouchier for helpful advice.

I would like to thank all the consultant physicians, anaesthetists and surgeons in the Royal Infirmary for allowing access to their patients. I would like to acknowledge gratefully the help from Sister $M$ Castle and the staff in the Dept of Medicine, Sister G Wilkie, Sister D Russell and her endoscopy staff for their help. Finally, I want to thank all the patients who took part in these studies without whose help these studies would not have been completed successfully.

I would like to dedicate this thesis to the memory of my grandfather who has been the source of my inspiration. 
The pathogenesis of portal hypertension in patients with cirrhosis is poorly understood and current treatment modalities are unsatisfactory. Transjugular intrahepatic portosystemic stent-shunt (TIPSS) is an interventional radiological technique involving the creation of a communication between the hepatic and the portal veins that is kept open using an expandable metal stent. Its introduction reduces the portal pressure which (a) helps in the management of portal hypertension and (b) allows us to study the contribution of portal pressure to the syndrome of cirrhosis.

The purpose of this thesis was to evaluate

(i) the effect of TIPSS on the natural history of cirrhosis

(ii) the changes in shunt function following its insertion and study the potential pathogenic mechanisms

(iii) the place of TIPSS in the management of variceal haemorrhage in patients with cirrhosis and

(iv) the use of TIPSS as a 'model' to study the pathogenesis of sodium retention in patients with cirrhosis.

The thesis aims to answer these questions by studying defined cohorts of patients with cirrhosis and portal hypertension undergoing TIPSS insertion for a variety of indications. The thesis is divided into various sections which deal with the above questions. The first section deals with the outcome of patients with cirrhosis treated with TIPSS and assesses the factors predicting mortality, rebleeding, encephalopathy and shunt insufficiency. It also prospectively 
assesses changes in liver function, neuropsychological profile, haematological profile and appraises their relationship with changes in the portal pressure. Studies in the next section were designed to evaluate the methods for assessment of shunt function and to study the pathogenesis of shunt insufficiency. The place of TIPSS in the management of variceal haemorrhage in patients with cirrhosis is evaluated in a randomised and controlled study in comparison with variceal band ligation. The last section looks at the mechanisms of sodium retention in cirrhosis particularly with reference to the 'hepatorenal reflex'. The final chapter is dedicated to discussing the findings of this study in the light of the available literature and define the areas of deficiency in our understanding and to outline future perspectives.

In conclusion, TIPSS is an exciting innovation which is relatively safe and can be performed in the vast majority of patients with cirrhosis with a very low procedure related mortality. Although its place in the management of uncontrolled variceal haemorrhage appears certain, further controlled studies are needed to assess its role in the secondary prophylaxis of variceal haemorrhage and in refractory ascites. 


\section{LEGENDS TO FIGURES}

Figure 1.1: Schematic representation of factors involved in the initiation and maintenance of sodium retention in patients with cirrhosis.

Figure 1.2: An approach to the management of ascites in patients with cirrhosis. OLT-Orthotopic Liver Transplantation, TIPSS-Transjugular intrahepatic portosystemic stent-shunt.

Figure 1.3: Schematic representation of the various factors involved in the pathogenesis of chronic hepatic encephalopathy.

Figure 1.3 (inset): Proposed mechanisms of neurotoxicity of $\mathrm{NH}_{3}$. Ammonia inhibits the cellular chloride channels which contribute to the depression of the CNS. Ammonia was thought to combine with $\alpha$-ketoglutarate, thereby depleting the CNS of intermediates of the citric acid cycle. This is unlikely because ammonia is metabolised in the brain by combination with glutamate to form glutamine but may affect energy metabolism by disturbing the malateaspartate shuttle. Ammonia facilitates the cerebral uptake of tryptophan which is a substrate for the generation of various neuroactive metabolites including serotonin. Ammonia reduces glutamatergic neurotransmission, causing neurodepression. 
Figure 2.1. This figure shows the steps involved in the insertion of TIPSS. (a) the guide wire is advanced into the middle hepatic vein (b) the stylet is advanced over the transjugular needle to puncture a branch of the portal vein and a guide wire is introduced into the splenic vein (c) an angioplasty balloon is used to dilate the track, the two waists represent the junction of the hepatic parenchyma and the hepatic and portal veins respectively, (d) a Wallstent has been deployed.

Figure 2.2. Kaplan Meier analysis of rebleeding in patients having TIPSS with the Palmaz stent or the Wallstent.

Figure 2.3. A dislocated Palmaz stent.

Figure 2.4. A Palmaz stent that has migrated from the parenchymal track into the trunk of the portal vein.

Figure 2.5. Hepatic vein stenosis producing recurrence of varices.

Figure 2.6. Intimal hyperplasia within a Wallstent.

Figure 2.7. A broken fragment of an angioplasty balloon stuck within the frame work of the Palmaz stents. This has been secured to the wall by insertion of a Wallstent within the Palmaz stents. 
Figure 2.8. Kaplan Meier analysis of mortality according to severity of liver disease.

Figure 2.9. Kaplan Meier analysis of shunt dysfunction according to the initial pressure gradient $(p<0.01)$.

Figure 2.10. Kaplan Meier analysis of shunt dysfunction according to the type of stent used.

Figure 2.11. Changes in the portal pressure gradient in the patients with TIPSS enrolled in the 'neuropsychology study.

Figure 2.12. Changes in alanine transaminase after TIPSS.

Figure 2.13. Changes in bilirubin after TIPSS.

Figure 2.14. Changes in Indocyanine clearance after TIPSS.

Figure 2.15. Changes in haemoglobin after TIPSS

Figure 2.16. Changes in reticulocytes in three patients with significant hemolysis 
Figure 2.17. Change in the portal pressure gradient and spleen size after TIPSS

Figure 2.18. Change in platelet count after TIPSS

Figure 3.1. This figure depicts the relationship between the portal pressure gradient and peak systolic velocity of blood in the portal vein.

Figure 3.2. Radiograph showing endocopy biopsy forceps introduced into the shunt through the sheath for obtaining biopsies of the occluding material.

Figure 3.3. Changes in portal pressure gradient in the group A patients.

Figure 3.4. Changes in the portal pressure in the group B patients.

Figure 3.5. Radiograph showing occluded Palmaz stents and a parallel Wallstent inserted through another hepatic vein.

Figure 3.6. Changes in portal pressure gradient in the Group C patients who were managed initially managed by balloon angioplasty and then by insertion of a parallel stent. Long term patency was obtained after insertion of the parallel stent. 
Figure 3.7. Changes in the portal pressure gradient in the Group $\mathrm{C}$ patients who were managed solely by balloon angioplasty. Note that there is predictable rise in the portal pressure gradient at subsequent assessment of shunt function.

Figure 3.8. Radiograph of an occluded stent where contrast fills the biliary system on forcible injection of contrast.

Figure 3.9. Cholangiogram of an explant liver demonstrating cicatrisation of the bile ducts in the vicinity of the shunt.

Figure 3.10. Photomicrograph of the histopathological appearance of a biopsy specimen obtained from an occluded shunt demonstrating biliary epithelium (positive staining for cytokeratin).

Figure 3.11. An endoscopic view of the shunt obtained from an explanted liver showing the continuous pseudo-intima.

Figure 3.12. Photomicrograph of the lining of the shunt showing the pseudointima.

Figure 3.13. Photograph of a shunt that has been dissected out of the liver showing that a major bile duct has been transected. 
Figure 3.14. Photograph of a shunt which has been dissected out and opened to demonstrate the occluding material. The arrow represents the primary thrombus that overlies the transected bile duct.

Figure 3.15 and 3.16. Photomicrograph of the histopathological appearance of the thrombus which overlies the transected bile duct demonstrating granulomatous inflammation and bile impregnation.

Figure 4.1. The outcome of patients with uncontrolled variceal haemorrhage in the TIPSS and oesophageal transection groups. Numbers within brackets refer to the number of patients.

Figure 4.2. Kaplan Meier analysis of survival in the TIPSS and transection groups. $p<0.05$.

Figure 4.3. Kaplan Meier analysis showing significantly less rebleeding $(p<0.0006)$ in the TIPSS group compared with the variceal band ligation group.

Figure 4.4. Kaplan Meier analysis of survival in the variceal band ligation and TIPSS groups $(p=n s)$.

Figure 4.5. Kaplan Meier analysis of shunt insufficiency after TIPSS 
Figure 5.1. Changes in urinary sodium following TIPSS.

Figure 5.2. Changes in creatinine clearance following TIPSS.

Figure 5.3. Changes in plasma renin activity following TIPSS.

Figure 5.4. Changes in Angiotensin II following TIPSS.

Figure 5.5. Changes in cyclic Guanosine mono phosphate (cGMP) following TIPSS

Figure 5.6. Changes in Atrial natriuretic peptide (ANP) following TIPSS.

Figure 5.7. Changes in Lithium Clearance following TIPSS.

Figure 5.8. The computer set-up and the thermodilution system that was used to measure renal and portal blood flow.

Figure 5.9. X-ray showing an inflated angioplasty balloon in the shunt inserted through the internal jugular vein and a reverse thermodilution catheter in the right renal vein inserted through the femoral vein.

Figure 5.10. Graph showing the changes in the renal blood flow prior to and following shunt occlusion. 
Figure 5.11. Graph showing the changes in cardiac output following shunt occlusion.

Figure 5.12. Relationship between portal blood flow and renal blood flow.

Figure 5.13. Relationship between changes in portal pressure gradient and changes in renal blood flow. 


\section{THE INDEX}

1.3. Pathophysiology of Portal Hypertension

1.31. Portal vascular resistance 27

1.311. Static phenomenon 27

1.312. Dynamic phenomenon 27

1.32. Hyperdynamic circulation 29

1.321. Increased vasodilatation 30

1.322. Vascular hyporeactivity 32

1.4. Complications of Portal Hypertension and their Management
1.41. Variceal haemorrhage 35
1.411. Development of varices 35
1.412. Risk factors for first variceal haemorrhage 35
1.413. Risk of first variceal haemorrhage 37
1.414. Prophylaxis from first variceal haemorrhage 40
1.415. Prognosis of acute variceal haemorrhage 41
1.416. Management of acute variceal haemorrhage 46
1.417. Risk of recurrent variceal haemorrhage 51
1.418. Secondary prophylaxis of variceal haemorrhage
1.419. Pitfalls in the assessment of prognosis 52 55 
1.42. Sodium retention, ascites and hepatorenal failure $\mathbf{5 6}$ 1.421. Pathogenesis 57

1.4211. Retention of Sodium 58

1.4212. Retention of water 60

1.4213. Hepatorenal syndrome 61

1.422. Principles of Management 62

1.423. Natural history and prognosis 65

1.43. Hepatic Encephalopathy (HE) 66

1.431. Pathogenesis of $\mathrm{HE} \quad 67$

1.4311. Blood brain barrier $\quad 68$

1.4312. Cerebral energy metabolism 68

1.4313. Effect of gut derived toxins $\quad 69$

1.4314. Cerebral neurotransmission $\quad 71$

1.4312. Principles of Management 73

1.44. Hypersplenism 78

1.5. Historical aspects of Transjugular intrahepatic portosystemic stent-shunt

Chapter 2 THE NATURAL HISTORY, MANIFESTATIONS AND PROGNOSIS OF TIPSS $\quad 85$

2.1. Aims 86

$\begin{array}{ll}\text { 2.2. Methods } & 87\end{array}$

2.21. TIPSS insertion, assessment of shunt function $\quad 87$

2.22. Patients and their follow up and evaluation 92

2.23. Statistical methods in the assessment of outcome and prognosis. $\quad 95$

2.24. Mental state and Neuropsychological function $\quad 97$

2.25. Haematological assessment 104 
2.31. TIPSS insertion 106

2.32. Results of patient follow up and evaluation 109

2.32. Results of analysis of prognostic variables in the prediction of variceal rebleeding, mortality, encephalopathy and shunt insufficiency following TIPSS. 109

2.32. Relationship between PPG and variceal haemorrhage 114

2.32. Increase in intracranial pressure after TIPSS 117

2.33. Changes in Neuropsychological and Liver function. 122

2.34. Results of Haematological assessment 129

2.4. Summary

132

Chapter 3 SHUNT DYSFUNCTION: METHODS OF FOLLOW UP, PROGNOSTIC FACTORS AND MECHANISMS OF SHUNT STENOSIS AND $\begin{array}{ll}\text { OCCLUSION } & 135\end{array}$

3.1. Comparison of Doppler ultrasonography and portography in $\begin{array}{ll}\text { the assessment of shunt dysfunction } & 136\end{array}$

3.11. Introduction and Aims 136

3.12. Methods 137

3.121. Study design 137

3.122. Portography and measurement of portal pressure

3.123. Doppler ultrasonography. 138

3.13. Results 140

3.14. Summary 141

3.2 Investigation into the pathogenesis of shunt insufficiency after TIPSS 
3.21 Introduction and Aims 143

3.22. Methods 144

3.221. Clinical follow up 144

3.222. Pathology 145

3.223. Radiology 146

3.23. Results 147

3.231. Clinical 147

3.233. Radiology 148

3.232. Pathology 148

3.24. Summary 151

Chapter 4 EVALUATION OF TIPSS FOR VARICEAL HAEMORRHAGE IN PATIENTS WITH CIRRHOSIS

152

4.1 Comparison of TIPSS with oesophageal transection in the management of uncontrolled variceal haemorrhage in cirrhosis

153

4.11. Introduction and Aims 153

4.12. Methods 154

4.121. Study Design 154

4.122. Patient population 154

4.123. TIPSS 158

4.125. Oesophageal transection 158

4.125. Definitions and Statistical analysis 158

4.13. Results 160

4.131. Mortality 160

4.132. Rebleeding 161

4.133. Encephalopathy 161

4.134. Others 162

4.14. Summary 164 
4.2 TIPSS Vs VBL in the secondary prophylaxis of variceal bleeding in patients with cirrhosis. A randomised and controlled study

4.21. Introduction and Aims 165

\subsection{Methods}

4.221. Patient Selection

4.222. Informed consent, Ethical approval, randomisation and study design

4.223. TIPSS insertion and follow up 168

4.224. Variceal band ligation and follow up 168

4.225. Follow up and treatment 169

4.226. Definitions and management of complications 169

4.227. Estimated cost of procedures 171

4.228. End points, Determination of sample size, study termination and statistical analysis

4.23. Results 174

4.231. Patients 174

4.232. Procedures 174

4.233. Rebleeding 177

4.234. Mortality 178

4.235. Encephalopathy 179

4.236. Shunt insufficiency 181

4.237. Sepsis 181

4.238. Cost of procedures 182

4.24. Summary 184

Chapter 5 TIPSS AS A TOOL TO STUDY THE RENAL DYSFUNCTION OF CIRRHOSIS 185

5.1 Mechanism of changes in renal function after TIPSS 186 
5.121. Patients 187

5.122. TIPSS procedure 187

5.123. Measurement of renal function 188

5.124. Measurement of changes in the Hormonal profile 188

5.125. Assessment of tubular function using Lithium Clearance 190

5.126. Analysis 191

5.13. Results 192

5.131. Patients 192

5.132. Changes in the renal function 192

5.133. Changes in hormonal profile 193

5.134. Changes in Lithium Clearance 193

5.135. Relationship between changes in renal function and liver function and the degree of portal hypertension 194

5.14. Summary 195

5.2 Use of TIPSS in the evaluation of the 'hepatorenal reflex' 196

5.21. Introduction 196

$\begin{array}{ll}\text { 5.22. Methods } & 198\end{array}$

5.221. Patients 198

5.222. Portography 200

5.223. Measurement of Portal blood flow 202

5.224. Measurement of changes in renal blood flow 202

5.225. Measurement of changes in cardiac output 203

5.226. Measurement of neurohumoral factors 204

5.227. Analysis 205

5.23. Results 206 
5.231. Changes in portal blood flow and its relationship with renal blood flow

5.232. Changes in renal blood flow and its relationship with the portal pressure gradient 206

5.233. Changes in Cardiac output 209

5.234. Changes in Neurohumoral factors

6.1. Shunt Dysfunction $\quad 215$

6.2. Mortality 221

6.3.Liver function and Encephalopathy $\quad 224$

6.4. Variceal Bleeding $\quad 228$

6.5. Sodium Handling 245

$\begin{array}{ll}\text { 6.6. Hepatorenal reflex } & 248\end{array}$

$\begin{array}{lr}\text { REFERENCES } & 255\end{array}$

PEER REVIEWED PUBLICATIONS RESULTING FROM WORK DONE FOR THIS THESIS 


\section{CHAPTER 1}

\section{INTRODUCTION}




\subsection{HISTORY OF PORTAL HYPERTENSION}

Stahl (Stahl et.al. 1748) was the first to describe a syndrome where the portal vein was diseased, the spleen was enlarged and there were large haemorrhoids. The association between bleeding from oesophageal varices and cirrhosis was first described by Fauvel (Fauvel et.al. 1858). Power (1840, quoted by Preble 1900) had however described bleeding from oesophageal varices before Fauvel, but he failed to recognise its association with liver disease. Preble (Preble et.al. 1900) reviewed 60 cases of fatal gastrointestinal haemorrhage due to cirrhosis and constructed the hypothesis that the fibrotic liver was the major cause of obstruction of portal circulation and that this is what led to increased pressure in the collateral circulation. $\mathrm{He}$ also made the fundamental observation that a third of these patients died of the first episode of variceal haemorrhage and that bleeding in the patients who survived was usually recurrent.

Herrick (Herrick et.al. 1907) studied the relationship between the hepatic arterial pressure and the portal pressure in animal models with normal and cirrhotic livers. He observed that portal pressure became elevated only when the arterial pressure was increased to $100 \mathrm{mmHg}$ in the animals with normal liver. On the other hand in animals with cirrhotic livers, a similar increase in portal pressure was observed when the arterial pressure was increased to $30 \mathrm{mmHg}$. He concluded that the high pressure in the hepatic artery was responsible for changes in the portal pressure. 
Mclndoe (Mclndoe et.al. 1928) first coined the term portal hypertension. Using techniques involving the injection of gelatin and Indian ink into the portal system he concluded that there was a progressive dissociation between the portal venous and hepatic arterial systems which resulted in the diversion of portal blood flow into the collateral channels. The hepatic artery therefore remained as the major blood supply of the hepatocytes which was progressively divorced from the normal portal blood supply. From these studies he concluded that the portal hypertension could be explained entirely on a physical basis due to a porto-hepatic venous outflow obstruction. Rousselot (Rousselot et.al. 1936) first described the existence of portal hypertension as a definite clinical entity by measuring the splenic venous pressure in a patient with Banti's disease.

\subsection{ANATOMY OF THE PORTAL VENOUS SYSTEM}

The portal system comprises of the veins that collect blood from the intra-abdominal part of the gastrointestinal tract, the spleen, the pancreas and the gallbladder. The portal vein is formed by the union of the splenic and the superior mesenteric veins, is approximately $6-8 \mathrm{~cm}$ long and enters the liver at the porta-hepatis as two main branches supplying the right and the left lobes. Inside the liver the portal veins are distributed segmentally.

The splenic vein is formed by the union of numerous splenic veins and the short gastric veins. This is then joined by the left gastro-epiploeic vein behind the body of the pancreas and then by numerous tributories draining 
the pancreas. The inferior mesenteric vein carries blood from the left part of the colon and the rectum and usually drains into the middle third of the splenic vein. The left gastric vein usually joins the main portal vein at its origin but may occasionally enter the splenic vein. The superior mesenteric vein is derived from numerous veins which drain the right side of the colon, the small intestine and the head of the pancreas.

Portal blood flow in man averages about 1 litre per minute and supplies about $60 \%$ of the oxygen demand of the liver (Tygstrup et.al. 1962). The rest of the liver blood flow is from the hepatic artery with a rate of flow of about $350 \mathrm{ml}$ per minute. The normal pressure in the portal venous system is about $5-7 \mathrm{mmHg}$. The splenic pulp pressure, which is an indirect measure of the pressure in the portal venous system is about $7-13 \mathrm{mmHg}$ and that in the hepatic veins is between 4 and $6 \mathrm{mmHg}$ (Richard PDI and Withrington PG, 1981b).

\section{The collateral circulation of portal hypertension}

When the pressure rises above $7 \mathrm{mmHg}$, the collateral circulation starts to develop between the high pressure portal system and the neighboring veins which are at a lower pressure, thereby permitting a diversion of portal blood into the systemic circulation. The actual site for the development of portal collateral vessels are those areas where veins draining into the portal system are in immediate juxtaposition to veins draining into the superior and inferior vena cava. These areas are the submucosa of the oesophagus and stomach, the submucosa of the rectum, the anterior abdominal wall and the left renal vein. Studies using cast 
erosion and radiological techniques have provided evidence that the collateral circulation that develops is due to the opening of pre-existing vasculature rather than a consequence of angiogenesis (Kitano et.al 1986, Vianna et.al. 1987).

\subsection{THE PATHOPHYSIOLOGY OF PORTAL HYPERTENSION}

Portal pressure is dependent upon the portal venous blood flow and the resistance offered by the hepatic sinusoids. Therefore, alteration to either the portal venous flow and/or the intrahepatic resistance would produce portal hypertension. The former is regulated by the splanchnic blood flow and the latter by neurohumoral mechanisms (Granger et.al. 1980). In the normal situation, physiological stimuli such as ingestion of food, exercise or Valsalva maneuver may alter splanchnic blood flow and therefore influence portal blood flow (Chou CC and Kvietys PR, 1981, Richard PDI and Withrington PG, 1981a). Sudden changes in portal pressure due to an increase in portal blood flow are prevented by homeostatic mechanisms that involve neurohumoral factors which serve to maintain the portal pressure within the normal range by modulating the portal vascular resistance (Richard PDI and Withrington PG, 1981b).

When these homeostatic mechanisms are disturbed by changes either in the portal venous inflow (forward hypothesis) or in the resistance (backward hypothesis), portal hypertension results (Witte CL and Witte MM, 1983). In order to decompress the portal system, portosystemic collaterals develop. Despite this, portal pressure remains elevated due to the 
development of a hyperdynamic splanchnic and systemic circulation that serves to maintain the enhanced portal venous inflow. It can therefore be appreciated that the forward and backward hypotheses are somewhat simplistic and that portal hypertension is the result of complex interplay between increased portal vascular resistance and blood flow. Factors contributing to changes in resistance and the hyperdynamic circulation of cirrhosis are described below.

\subsection{Portal Vascular Resistance}

This has a static and a dynamic component.

\subsection{Static Phenomenon}

The proposed pathogenic mechanisms involved in the development of the static or the fixed component of portal hypertension has evolved over the years. Mclndoe et.al. (Mclndoe et.al. 1928) suggested that portal hypertension was due to diffuse hepatic fibrosis. It was then hypothesised that it resulted from the displacement of intrahepatic vasculature by cirrhotic nodules (Hart GF and Lisa GR, 1937, Kelty et.al. 1950, Popper et.al. 1952) and finally fibrosis in the space of Disse was observed (Popper et.al. 1961). In alcoholic subjects the degree of fibrosis of the space of Disse has been shown to correlate directly with the severity of portal hypertension (Orrego et.al. 1979, Orrego et.al. 1981). Another theory proposes that hepatocyte enlargement as a result of excessive intracellular water and fat accumulation may be responsible for increased intrahepatic sinusoidal pressure (Blendis et.al. 1982, Crossley et.al. 1985). This hypothesis is supported by the 
observation that portal pressure fluctuates widely with alcohol intake or abstinence (Leevy et.al 1970, Reynolds et.al. 1960).

When the hepatocyte enlargement theory and the collagenisation of the space of Disse theory, were put to test in alcoholic and non alcoholic subjects the results favored the hepatocyte enlargement theory as the primary factor (Blendis et.al. 1982), although the interaction between these two mechanisms are almost inseparable as an elevation in intrahepatic pressure is associated with cell ballooning which may in fact stimulate fibrogenesis (Crossley et.al. 1985).

\subsection{Dynamic Phenomena}

The demonstration that there is a dynamic component of intrahepatic portal hypertension opened up exciting prospects for treatment. The hepatic vasculature is richly endowed with adrenergic receptors and this is subject to complex neurohumoral control (McClain et.al. 1989, MacMathuna et.al. 1990a, Biolac-Sage et.al. 1990). Studies using calcium channel antagonists (Reichen $\mathrm{J}$ and Le $\mathrm{M}, 1986$ ) and clonidine ( $\alpha_{2}$ agonist) (Willet et.al. 1986) in isolated rat liver and in cirrhosis showed that intrahepatic resistance was amenable to pharmacological manipulation. The action of these neurohumoral substances may be exerted at the level of the myo-fibroblasts present within the capillarised sinusoids, the receptors on the blood vessels or even on the sinusoid cells (Bhathal PS and Groszmann RJ, 1985). The response to neurohumoral substances in health is very different from that seen in liver disease primarily because the disease tends to alter receptor function and modifies the response to vasoactive agents (Ballet et.al. 1988, 
Marteau et.al. 1989). This observation is further supported by the relatively contradictory finding of the opposing actions of noradrenaline on the portal system (predominantly vasoconstrictive), and upon the systemic circulation where there is a markedly reduced sensitivity to the circulating noradrenaline (Kiel et.al. 1985).

The initial finding of alteration in intrahepatic vascular resistance with the use of calcium channel antagonists was not confirmed in later studies (Marteau et.al. 1989). This discrepancy may in part be explained by a compensatory mechanism whereby any reduction in intrahepatic vascular resistance due to verapamil is neutralised by an increase in portal venous flow as a result of systemic vasodilatation. Nitrates have been shown to reduce intrahepatic vascular resistance in cirrhotic rat models (Blei et.al. 1988) and this has been confirmed by Navasa and his colleagues using isosorbide mononitrate in patients with cirrhosis (Navasa et.al. 1988). This action of nitrates is probably mediated by nitric oxide which acts via stimulation of cyclic GMP (Murad et.al. 1986). The role for other important vasoconstrictors such as platelet activating factor (Richardson PDI and Withrington PG, 1976) and endothelin (Benoit et.al. 1986, Lee et.al. 1988, Pizcueta et.al. 1990) has been observed both in animal models and in the cirrhotic patient.

\subsection{Hyperdynamic Circulation}

The systemic circulation in patients with cirrhosis is characterised by peripheral vasodilatation occurring as a result of a reduction in splanchnic vascular resistance and an increase in the cardiac output which contributes 
to increase in the portal venous inflow. This increase in the portal venous inflow may therefore contribute to the portal hypertension (forward hypothesis).

This hyperdynamic state is probably due to a combination of increased levels of circulating endogenous vasodilators and a reduced vascular sensitivity to vasoconstrictor compounds.

\subsection{Vasodilatation}

Glucagon concentration is elevated in the circulation of patients with cirrhosis and reduces vascular sensitivity to vasopressor agents and increased splanchnic blood flow. It does this by activating adenylate cyclase (Richard PDI and Withrington PG, 1976, Lee et.al. 1988, Pizcueta et.al. 1990). Inability to reduce portal blood flow using glucagon antiserum suggests that glucagon is not the only factor which is responsible for the circulatory abnormalities (Benoit et.al. 1986).

Portosystemic shunting induces endotoxaemia in patients with cirrhosis and this could induce nitric oxide synthase activity in the endothelium and vascular smooth muscle cells. Nitric oxide is formed from catabolism of L-arginine which acts by stimulation of cyclic guanosine monophosphate (cGMP) (Vallance P and Moncada S, 1991, Moncada S and Higgs $A, 1993)$. In addition to its constitutive activity in the endothelium which is involved in the regulation of normal vascular tone, in vitro studies have implicated nitric oxide in the vascular hyporeactivity of portal hypertension (Seiber CC and Groszmann RJ, 1992, Seiber et.al. 1993, Castro et.al. 1993) and studies in animal models have shown that portal 
blood flow and pressure are reduced following inhibition of nitric oxide synthase activity (Pizcueta et.al. 1992a, Pizcueta et.al. 1992b). Experiments in patients with cirrhosis and controls using measurement of forearm blood flow after inhibition of nitric oxide synthase showed no significant difference in vascular responses (Calver et.al. 1994). These observations suggest that nitric oxide mediated tone is not solely responsible for the changes in the portal circulation.

Adenosine has been implicated in the circulatory disturbances of cirrhosis and is formed by dephosphorylation of adenosine triphosphate (ATP) which is often co-released with noradrenaline from the nerve endings. It acts via the adenosine II receptor. An increase in cyclic GMP activity in the vascular smooth muscle cells and the endothelial cells initiates nitric oxide production (Moritoki et.al. 1990, Rose'Meyer RB and Hope W, 1990). Blockade of adenosine receptors in animal models of cirrhosis reduces portal blood flow but does not produce any change in the portal pressure (Lee et.al. 1992). It appears unlikely that adenosine is the sole determinant of the initiation of the vasodilatation in cirrhosis although it may play a role in its maintenance (MacMathuna et al 1990b).

Atrial natriuretic peptide is produced in response to atrial stretch as a result of increasing blood volume and vasodilates through its action on guanylate cyclase leading to increased formation of cGMP (Epstein et.al. 1988). Changes in the levels of cGMP measured in patients with cirrhosis is inconsistent (Hayes et.al. 1992, Burghardt et.al. 1986). A recent study has 
however shown a good correlation between the haemodynamic changes of cirrhosis and levels of measured cGMP (Burghardt et.al. 1986).

Prostacyclin is a potent dilator of the peripheral vasculature and has been shown to be elevated in patients with cirrhosis (Guarner et.al. 1992). It is produced by the action of cyclooxygenase on arachidonic acid. It is released from the endothelium in response to portal vein distension, endotoxin and vasopressors (Wu et.al. 1991, Guarner et.al. 1992). Inhibition of cyclooxygenase activity reduces portal blood flow and pressure although do not reach normal levels (Wu et.al. 1993, Oberti et.al. 1993). Vasoactive intestinal peptide and substance $P$ have been shown to be elevated in various studies, but their role in the pathogenesis of the hyperdynamic circulation of cirrhosis is not clear (Hunt et.al. 1979, Ring-Larsen et.al. 1986). Bile acids have vasodilatory properties and are elevated in patients with cirrhosis and seem to play an important role in the development of the hyperdynamic circulation, although no correlation has been observed between its concentration and the severity of circulatory derangement (Ohkibuda et.al. 1984). Similarly, tumour necrosis factor has been thought to be an important mediator of the vascular changes but its inhibition did not shown any significant change in portal pressure (Soupison et.al. 1994). Both gamma-aminobutyric acid and opioids are known to be potent vasodilators and their concentrations have been found to be elevated in cirrhosis. Their role in the vasodilatation of portal hypertension remains unclear because of variable effects from their inhibition (Lee et.al. 1987, Minuk et.al. 1988).

\subsection{Vascular hyporeactivity}


In vivo and in vitro studies have shown that the vascular smooth muscle in cirrhosis is hyporeactive to noradrenaline and angiotensin II, implying that the defect lies either at the receptor level or at the post receptor level in the smooth muscles (Keil et.al. 1985, MacGilchrist et.al. 1991).

Measurement of noradrenaline in the serum reflects sympathetic nerve activity (Henriksen et.al. 1984). Patients with cirrhosis clear noradrenaline from their plasma normally, therefore the elevated levels reflect over-production and enhanced sympathetic activity (Henriksen et.al. 1984). The action of noradrenaline on the smooth muscles is through the alpha-1 and alpha- 2 receptors. These are more important in the veins than in the arteries. The number of alpha- 2 receptors in patients with cirrhosis is normal. Therefore it is difficult to explain why there is an inhibition of the action of noradrenaline despite increased levels of noradrenaline with normal numbers of receptors.

Angiotensin II is a potent vasoconstrictor, and acts on the angiotensin I receptors on the smooth muscles. Its concentration in cirrhosis is known to be elevated and the use of its antagonists produces dramatic reduction in blood pressure. Very little is known about the status of its receptors in cirrhosis.

Endothelin, is also a potent vasoconstrictor and is produced by the endothelium of the blood vessels in response a number of stimuli. Its concentration is elevated in patients with cirrhosis and ascites and may also be elevated in patients without ascites (Asbert et.al.1993, Moller et.al.1993). It acts through its receptors (endothelin receptor $A$ and $B, E T_{A}, E T_{B}$ ) and the 
status of these receptors in patients with cirrhosis is unknown. Use of endothelin antagonists has been shown to reduce portal pressure probably by reducing intrahepatic resistance (Asbert et.al.1993, Moller et.al.1993).

Serotonin is an arterial vasodilator and acts through the 5 hydroxy tryptamine- 1 receptor on the endothelial cells. Its actions on the veins is quite different because it acts through the 5 hydroxy tryptamine- 2 receptor and produces venous constriction (Peroutka et.al. 1990, Vanhoutte et.al. 1990). Administration of ketanserin (a $5 \mathrm{H} 2$ antagonist) causes reduction in the portal pressure gradient and reduction in collateral blood flow (Hadengue et.al. 1987). Chronic administration of this drug produces reduction in portal pressure gradient but does not return it to normal levels (Vorobioff et.al. 1989).

Vasopressin is released non osmotically from the hypothalamus and acts upon the vascular smooth muscles via its receptors to cause vasoconstriction. It has been shown to be elevated in patients with cirrhosis probably as a compensatory mechanism for the reduced systemic vascular resistance (Claria et.al. 1991).

Platelet activating factor increases hepatic vascular resistance and may contribute to portal hypertension. Its effects have not been fully studied in patients with cirrhosis (Lapointe et.al.1989).

In summary, despite marked activation of the vasopressor system the peripheral circulation remains vasodilated and this has been thought either to be related to a defect at the receptor level which seems unlikely or to an 
alteration in the mechanisms of signal transduction following the interaction of vasopressor with its receptor. The major control of signal transduction following receptor binding is through cyclic nucleotides such as cyclic adenosine monophosphate (cAMP) and cGMP or through phosphatidyl inositol turnover. A study of cyclic nucleotides in the platelets (reflects vascular smooth muscle activity) from cirrhotic patients indicated an excess of CAMP and of cGMP with impairment of phosphatidyl inositol degradation (Laffi et.al. 1993). Further studies are required to characterise the nature of these defects.

\subsection{COMPLICATIONS OF PORTAL HYPERTENSION}

The most important complications of portal hypertension in patients with cirrhosis are

1. Variceal haemorrhage

2. Sodium retention, ascites and hepatorenal failure

3. Chronic hepatic encephalopathy

4. Hypersplenism

The following section deals with the pathogenesis, natural history, prognosis and the presently available modalities of treatment that are available to manage this condition at this present time.

\subsection{VARICEAL BLEEDING}

\subsection{Development of varices}


The rise in portal pressure is associated with the development of collateral circulation which allows the portal blood to be diverted into the systemic circulation in an attempt to decompress the portal system. These spontaneous shunts occur a) at the cardia through the intrinsic and extrinsic gastro-oesophageal veins b) in the anal canal where the superior haemorrhoidal vein belonging to the portal system anastomoses with the middle and inferior haemorrhoidal veins which belong to the system circulation $\mathrm{c}$ ) in the falciform ligament of the liver through the paraumbilical veins which are the remains of the umbilical circulation of the foetus, d) in the abdominal wall and the retroperitoneal tissues, where veins and abdominal organs are in juxtaposition including veins from the liver to the diaphragm, veins in the splenorenal ligament, in the omentum and lumbar veins, and e) blood diverted from diaphragm, gastric, pancreatic, splenic, and adrenal may drain into the left renal vein (Mikkelson et.al. 1965).

There are considerable data in the literature which suggest that varices develop and enlarge with time. Christensen (Christensen et.al. 1981) followed a cohort of 532 patients with cirrhosis over 12 years and showed that the cumulative percentage of patients with varices over this period increased from $12 \%-90 \%$. In a study involving 80 patients followed for 16 months Cales (Cales et.al. 1989) showed that $20 \%$ patients who did not have varices developed new varices and $42 \%$ patients with small varices showed definite enlargement. Czaja (Czaja et.al. 1979) on the other hand showed that the prevalence of varices increased from $8 \%-13 \%$ over 5 years in a cohort of patients with chronic active hepatitis treated with prednisolone. 
The two factors that appear to determine the development of varices are continued hepatic injury and the degree of portosystemic shunting. Evidence for the former is derived from studies in which varices were shown to regress with time. Baker (Baker et.al. 1959) followed a cohort of 112 patients with oesophageal varices and showed that varices had disappeared in 9 patients, regressed in 7 and remained unchanged in 96 . He concluded that the disappearance and regression of varices may be related to abstinence from alcohol. This observation was confirmed in a study by Dagradi (Dagradi et.al. 1972) who followed a cohort of patients with alcoholic cirrhosis over 3 years and showed a reduction in variceal size in 12 of the 15 patients with alcoholic cirrhosis who stopped drinking and an enlargement in variceal size in 17 patients who continued to drink. Cales (Cales et.al. 1989) showed that $16 \%$ of patients with alcoholic cirrhosis who continued to imbibe alcohol showed regression in varices.

\subsection{Risk factors for first variceal bleeding}

The factors that predispose to and precipitate variceal haemorrhage are still not clear, although the venous anatomy, portal pressure, intravariceal pressure, variceal size, endoscopic appearances of the varices (red spot, weals), tension on the variceal wall and severity of the underlying liver disease have been blamed. The most important factors that are thought to increase the risk of variceal bleeding are (i) pressure within the varix (ii) variceal size (iii) tension on the variceal wall and (iv) severity of the liver disease.

Portal Pressure 
In most cases, the portal pressure reflects the intravariceal pressure (Dawson et.al. 1985) and a hepatic venous pressure gradient greater than 12 $\mathrm{mmHg}$ is necessary for the development of and bleeding from oesophageal varices although no linear relationship exists between the severity of portal hypertension and the risk of variceal haemorrhage (Lebrec et.al. 1980, Garcia-Tsao et.al. 1985). However, the HVPG tends to be higher in the bleeders as well as in patients with larger varices. In a prospective study comparing propranolol with placebo for the prevention of first variceal haemorrhage, Grosszmann (Grosszmann et.al. 1990) showed that bleeding from varices did not occur if the portal pressure gradient could be reduced to less than $12 \mathrm{mmHg}$. This pressure has since been accepted as the aim of pharmacological therapy of portal hypertension.

\section{Variceal Size}

This is best assessed endoscopically and variable results in the literature are because of the lack of definition regarding the distinction between large and small varices. Numerous studies (Lebrec et.al. 1980, Vinel et.al. 1986) have shown that the risk of variceal haemorrhage increases with the increase in the size of varices (Palmer and Brick 1956).

\section{Varix Wall Structure and Tension}

Polio and Grosszmann (Polio J and Grosszmann RJ, 1986) using an in vitro model showed that rupture of varices was related to the tension on the variceal wall. The tension depends upon the radius of the varix. In this model, increasing the size of the varix and decreasing the thickness of the variceal wall caused variceal rupture. Therefore the tension on the variceal 
wall was increased by variceal pressure, size of the varix and a decrease in wall thickness.

Endoscopic features such as "red spots" and "weal" markings were first described by Dagradi (Dagradi et.al. 1972). They have been described as being important in the prediction of variceal haemorrhage. These features represent changes in variceal wall structure and tension associated with the development of microtelangiectasias. In a retrospective study by the Japanese Research Society for Portal Hypertension Beppu et.al. (Beppu et.al.1981) showed that $80 \%$ patients who had blue varices or cherry red spots bled from varices suggesting that this was an important predictor of variceal haemorrhage in cirrhosis.

Severity of liver Disease and Bleeding indices

Two independent groups assessed factors predicting first variceal haemorrhage in cirrhosis prospectively. First, the North Italian Endoscopic Club (NIEC) (NIEC 1988) reported their findings in 1988 and was followed in 1990 by data from the Japanese (Inokuchi, 1990). Both these studies showed that bleeding risk was based upon 3 factors. These were severity of liver disease as gauged by Child class, variceal size and red weal markings. NIEC study showed a very wide range in the risk of bleeding from $6 \%-76 \%$ depending upon the presence or the absence of the different factors. This index was prospectively validated in a study by Prada (Prada et.al. 1994). Using the same variables the NIEC index was simplified by DeFranchis (DeFranchis et.al. 1991) and shown to correlate with the original index. Further studies showed that the HVPG and intravariceal pressure were also 
independent predictors of first variceal haemorrhage when analysed in conjunction with the NIEC index (Merkel et.al. 1992).

In summary, the risk of first bleed from varices in cirrhosis is dependent predominantly upon the portal pressure, tension on the variceal wall, presence or the absence of the red sign and the severity of liver disease. Unfortunately, these data have been derived from studies that assess changes at one given time point only. There are very few studies on the evolution with time. It has been suggested from looking back at the old shunt trials that ascites increases the risk of bleeding.. Continued alcohol abuse and the rate of deterioration in the severity of liver disease also increases the risk of bleeding (Burroughs et.al. 1989, Cales et.al. 1989, Dagradi et.al. 1972).

\subsection{Risk of first variceal bleed}

Data describing the overall risk of bleeding from varices must be viewed with caution and has some pitfalls in interpretation. The natural history of patients who have varices diagnosed as a part of their work up is different from patients who have complications of liver disease. Patients in trials may represent a different population from the patients who have had documented varices and not bled during follow up. Most studies do not comment upon either the severity of liver disease or upon whether patients with alcoholic cirrhosis are continuing to drink or not as both these factors have a significant effect upon the risk of variceal haemorrhage.

Most studies report bleeding from varices in about $20-50 \%$ during the period of follow up. Baker (Baker et.al. 1959) reported variceal bleeding in 33 
of the 115 patients that he followed for a mean of 3.3 years with a mortality of $48 \%$ from first variceal haemorrhage. These data were confirmed by Christensen (Christensen 1981). About $70 \%$ episodes of bleeding occur within 2 years of diagnosis.

Prophylactic propranolol trials also show results similar to those of the shunt trials with most of the episodes of bleeding occurring within the first 2 years of follow up. In these studies the rate of first variceal haemorrhage varied from $22 \%$ to $61 \%$ (Pascal et.al. 1987, Ideo et.al. 1988, Lebrec et.al. 1988, IMPP 1989, Conn 1991). This vast difference in the rate of first bleed relates almost certainly to the number of patients with severe liver disease included in the study (Pascal, Child C-46\%, bleeding-61\%; IMPP, Child C$6 \%$, bleeding-32\%; Conn, Child C-6\%, bleeding-22\%). Mortality varied from $24 \%$ to $49 \%$ over 2 years (Pascal, mortality-49\%; IMPP, mortality-24\%; Conn, bleeding-24\%). This was also almost certainly related to the severity of liver disease.

\subsection{Prophylaxis from variceal haemorrhage}

As stated earlier 30 to $50 \%$ of patients with portal hypertension will bleed from varices and about $50 \%$ will die from the effects of the first bleed and it seems rational to develop prophylactic regimes to prevent the development of and bleeding from these varices. One factor that emerges very clearly from looking at the trials of prophylaxis of variceal haemorrhage in cirrhosis is that most of the published trials do not have sufficient power to identify favorable treatment effects. Based upon the expected bleeding and death rates in the control group the minimum number of patients needed to detect a 
$50 \%$ reduction in bleeding would be 270 patients, and 850 patients to detect the same reduction in mortality.

Surgery

Portacaval shunts: There are 4 trials in the literature which have randomised a total of 302 patients (Conn et.al. 1965, Jackson et.al. 1968, Resnick et.al. 1969, Conn et.al. 1965) either to prophylactic shunt surgery or to non-active treatment. A meta-analysis of these studies showed a significant benefit in the reduction of variceal bleeding (odds ratio (OR) 0.31 , $95 \%$ confidence intervals $(\mathrm{Cl}) 0.17-0.56)$, significantly greater risk of hepatic encephalopathy $(\mathrm{HE})(\mathrm{OR} 2,95 \% \mathrm{Cl} 1.2-3.1)$ and of mortality $(\mathrm{OR} 1.6,95 \% \mathrm{Cl}$ 1.02-2.57) in patients treated with shunt surgery (D'Amico et.al. 1995).

Devascularisation procedures: Inokuchi (Inokuchi et.al. 1990) showed that there was a significant reduction in variceal bleeding and in mortality in patients treated with a variety of devascularisation procedures. There are however, numerous problems with the interpretation of this study because of the use of different procedures in each of the 22 centres. These results need confirmation in a further study.

\section{Pharmacologic therapy}

The mainstay of pharmacologic approach to the primary prophylaxis of variceal haemorrhage has been propranolol which reduces the portal pressure gradient (Reichen J, 1990), reduces azygos blood flow and also variceal pressure (Bosch et.al. 1984). It achieves this by causing splanchnic vasoconstriction, and reducing cardiac output. 
There are 9 randomised trials assessing its effectiveness; 7 are fully published papers and 2 are in the abstract form (Pascal et.al.1987, Ideo et.al. 1988, Lebrec et.al. 1988, IMPP 1989, Andreani et.al. 1990, Conn et.al. 1991, PROVA 1991, Strauss et.al. 1988, Colman et.al. 1990). The risk of variceal bleeding was lower in 7 studies (Pascal et.al.1987, Ideo et.al. 1988, Lebrec et.al. 1988, IMPP 1989, Andreani et.al. 1990, Conn et.al. 1991, Strauss et.al. 1988), significantly lower in 4 studies (Ideo et.al. 1988, IMPP 1989, Andreani et.al. 1990, Conn et.al. 1991) and unchanged in 1 study (PROVA 1991). There was a higher incidence of bleeding in the propranolol group in 1 study. This was a small study and unbalanced randomisation is likely because of a very low bleeding rate in the control group (Colman et.al. 1990). Mortality was reduced in 7 trials (Pascal et.al.1987, Ideo et.al. 1988, IMPP 1989, Andreani et.al. 1990, Conn et.al. 1991, PROVA 1991, Strauss et.al. 1988) significantly in 1 (Pascal et.al.1987) and unchanged in 2 (Lebrec et.al. 1988, IMPP 1989). A meta-analysis showed that the risk of rebleeding was significantly lower (OR $0.54,95 \% \mathrm{Cl} 0.39-0.74)$ although no significance was detected in differences in mortality (OR $0.75,95 \% \mathrm{Cl} 0.57-1.06$ ) (D'Amico et.al. 1995).

Interest in the use of vasodilators such as iso-sorbide mononitrate has grown since the demonstration that it reduces portal pressure as effectively (Navasa et.al. 1989) as propranolol. A trial comparing iso-sorbide mononitrate with propranolol showed no significant difference between these agents (Angelico et.al. 1993).

Endoscopic therapy 
Sclerotherapy: There are 19 trials comparing endoscopic variceal sclerotherapy with no treatment of which 4 are in the abstract form (Andreani et.al. 1990, PROVA. 1991, Strauss et.al. 1988, Paquet et.al. 1982, Witzel et.al. 1985, Koch et.al. 1986, Kobe et.al. 1990, Wordehoff et.al. 1987, Santangelo et.al. 1988, Sauerbruch et.al. 1988, Piai et.al. 1988, Potzi et.al. 1989, Russo et.al. 1989, Triger et.al. 1991, VACVSG 1991, De Franchis et.al. 1991, Saggioro et.al. 1986, Fleig et.al. 1987, Planas et.al.). These trials include 1630 patients and these studies are significantly heterogeneous. Ten of the trials included only patients with large varices; the other 9 include patients with varices of any size. Various sclerosants were used at different doses and injected intra or para variceally. The results of these trials are very variable with 2 studies showing reduction in both bleeding and mortality (Paquet et.al. 1982, Witzel et.al. 1985), 1 study showing a reduction in mortality but no difference in rebleeding (Potzi et.al. 1989), I study showing a significant increase in the risk of bleeding (Santangelo et.al. 1988) and another significantly greater mortality (VACVSG 1991). Due to marked heterogeneity between these studies a meta-analysis is clinically useless. At the present time sclerotherapy cannot be recommended for prophylaxis of variceal haemorrhage in patients with cirrhosis.

Variceal band ligation: Sarin et.al. (Sarin et.al. 1996) compared variceal band ligation with no active treatment in a randomised study and showed that there was a significant reduction in variceal bleeding in patients treated with band ligation. No significant effect on mortality was found. This 
treatment needs to be compared with the present gold standard, i.e. propranolol.

\subsection{Prognosis of acute variceal haemorrhage}

The average mortality of the first episode of variceal bleeding in most studies is $50 \%$. Mortality from variceal haemorrhage is related closely to the severity of liver disease (Lebrec et.al. 1984, Burroughs et.al. 1983, Villeneuve et.al. 1986, Colombo et.al. 1989, Garden et.al. 1990). Over a mean follow up of 1 year, the average mortality for subsequent variceal haemorrhage is $5 \%$ in Child class A patients, $25 \%$ in Child class B patients and $50 \%$ in Child class $\mathrm{C}$ patients. Although serum creatinine has been shown in some studies to predict overall survival (Garden et.al. 1985, Christensen et.al. 1989), Child class is superior to any other predictive factor in determining mortality within 6 weeks or 30 days of the initial haemorrhage.

Vinel et.al. (Vinel et.al. 1986) showed that HVPG was predictive of survival when this was measured at 2 weeks after the acute bleed. However, it is unclear whether this was independent of the severity of liver disease. Whether active bleeding at the time of endoscopy predicts mortality is not clear. Although Cardin et.al. (Cardin et.al. 1990) found that this was an important factor, Balanzo et.al. (Balanzo et.al. 1991) could not confirm this finding. Active bleeding at the time of endoscopy does however predict early rebleeding (Siringo et.al. 1991). Risk of death decreases quickly after admission such that the risk of death becomes virtually constant about 6 weeks after bleeding (Graham DY and Smith JL, 1981, Burroughs et.al. 1989). 


\subsection{Management of acute variceal bleeding}

The most important step in the management of acute variceal haemorrhage is the initial resuscitation and the protection of the airway to prevent aspiration. Early endoscopy allows examination of the upper gastrointestinal tract enabling accurate diagnosis of the bleeding site and decision regarding the management. The following measures of controlling the bleeding are presently available.

\section{Pharmacological Therapy}

The two major classes of drugs that have been used in the control of acute variceal bleeding are vasopressin or its analogues, either alone or in combination with nitroglycerine, and somatostatin or its analogues.

Vasopressin reduces portal blood flow, portal systemic collateral blood flow and variceal pressure (Reichen 1990). It does however have significant systemic side effects such as increase in peripheral resistance, reduction in cardiac output, heart rate and coronary blood flow. In comparison with no active treatment, the pooled results of 4 randomised trials (Merigan et.al. 1962, Conn et.al. 1975, Mallory et.al. 1980, Fogel et.al. 1982) showed that it reduced failure to control variceal bleeding although mortality was unaffected. Five trials have compared sclerotherapy with vasopressin and although 1 study showed significant effect upon reduction in the failure to control the variceal bleed, no significant differences between these groups were found in the overall analysis. However, rebleeding was significantly lower in patients treated with sclerotherapy. 
The addition of nitroglycerin enhances its effects on the portal pressure and reduces the cardiovascular side effects (Grosszman et al 1982). Three randomised trials compared vasopressin alone against vasopressin and nitroglycerine (Tsai et.al. 1986, Gimson et.al. 1986, Bosch et.al. 1989) and the pooled data from these showed that the combination was associated with a significant reduction in the failure to control bleeding although no survival benefit was demonstrated.

Glypressin which is a synthetic analogue of vasopressin has an immediate vasoconstrictor action followed by haemodynamic effects due to slow conversion to vasopressin. Its efficacy has been found in 3 placebo controlled trials to significantly reduce the failure to control bleeding significantly and also to improve survival (Freeman et al 1989 and Soderlund et al 1990, Levacher et.al. 1994). Three randomised trials compared its efficacy against vasopressin alone in 3 (Freeman et.al. 1989, Desaint et.al. 1987, Chiu et.al. 1990) and 2 compared it with vasopressin in combination with nitroglycerine (Lee et.al. 1988, D'Amico et.al. 1994). Glypressin reduced failure to control bleeding significantly when compared with vasopressin alone and was as good as the combination of vasopressin and nitroglycerine. It was however shown to be safer to use. Three trials compared it with somatostatin and found it to be equally effective (Silvain et.al. 1993, Walker et.al. 1992, VBSG 1993). Two trials compared its efficacy against balloon tamponade and found it to be equally effective (Colin et.al. 1987, Fort et.al. 1990). 
Somatostatin causes selective splanchnic vasoconstriction and reduces portal pressure and portal blood flow (Bosch et al 1981). It was shown to significantly reduce the failure to control bleeding in one trial (Burroughs et.al. 1990) and did not show any significant differences against placebo in another (Valenzuela et.al. 1989). Seven trials (Kravetz et.al. 1984, Jenkins et.al. 1985, Bagarani et.al. 1987, Cardona et.al. 1989, Hsia et.al. 1990, Saari et.al. 1990, Rodriguez-Moreno et.al. 1991) compared its efficacy with vasopressin and showed that somatostatin reduced the failure to control rebleeding and was associated with significantly fewer side effects.

Three trials compared somatostatin with balloon tamponade and showed that these were equally effective in reducing the failure to control variceal bleeding (McKee 1990, Jaramillo et.al. 1991, Avgerinos 1991). Five trials have compared somatostatin or its analogue, octreotide with sclerotherapy (DeFebo et.al. 1990, Jenkins et.al. 1992, Sheilds et.al. 1992, Planas et.al. 1994, Sung et.al. 1993) and have shown no significant differences in either the failure to control bleeding, rebleeding and mortality.

Data from a large randomised trial comparing octreotide with placebo was presented at a recent meeting and showed conclusively that there was no significant difference between the two groups either in terms of reduction in the failure to control bleeding, amount of blood transfused and mortality at 42 days or at 90 days (Burroughs AK, 1996).

\section{Endoscopic Therapy}

Sclerotherapy: Endoscopic variceal sclerotherapy using a flexible endoscope with sclerosants is based upon the concept that bleeding from varices is 
stopped by thrombosis of the bleeding varix secondary to either an intravariceal or a paravariceal injection of a sclerosant which is an oily ester. When assessing trials of sclerotherapy in acute bleeding there is enormous variation in the type of sclerosant used, the experience of the operator, whether intravariceal or paravariceal injections was used and what the schedule of follow up was. Furthermore, the interpretation of the results of trials comparing injection sclerotherapy with non invasive therapy is complicated by the inclusion of patients who were not actively bleeding at the time of randomisation (Soderlund et al 1985, Larson et al 1986). Four trials have compared sclerotherapy with balloon tamponade (Barsoum et.al. 1982, CEVSP 1984, Paquet KJ and Feusener F, 1985 and Moreto et al 1988) and 2 of these showed a significantly higher control of bleeding in patients treated with sclerotherapy (Paquet KJ and Feusener F, 1985 and Moreto et al 1988). The results of control of bleeding in the sclerotherapy patients are unrealistic at $95 \%$ and $100 \%$ respectively.

Sclerotherapy has been compared with oesophageal transection in 4 randomised trials (Cello et.al. 1982, Huizinga et.al. 1985, Teres et.al. 1987b, Burroughs et.al. 1989) and with the portacaval shunt in 1 (Cello et.al. 1987) in patients who had variceal haemorrhage that was uncontrolled. Although all the studies showed a reduction in failure to control bleeding in patients treated with surgical therapy, this reached statistical significance in one (Burroughs et.al. 1989). Rebleeding was also significantly higher in the sclerotherapy group. Although there were no differences in mortality, the rate 
of development of encephalopathy was significantly higher in patients treated with the surgical shunt.

\section{Variceal Band Ligation}

This technique is a modification of that used for the elastic band ligation of internal haemorrhoids. Its use in humans was first described in 1988 (Stiegmann GV and Goff JS, 1988) and a subsequent randomised clinical trial comparing banding with sclerotherapy showed a significant reduction in the rate of complications and improvement in survival (Stiegmann GV and Goff JS, 1992). Another trial compared these two forms of treatment and confirmed that banding controls active variceal haemorrhage and no significant differences were found in the control of active bleeding in the 2 groups. This trial showed a reduction in the rates of rebleeding in the band ligation group. There was however no survival benefit (Gimson et al 1993).

\section{Other Endoscopic Measures}

Control of bleeding using tissue adhesives has been reported in about $90 \%$ of cases (Ramond et al 1986, Soehendra et al 1987). There were however similar rates of re-bleeding as with sclerotherapy and there were significant complications in the form of cerebrovascular accidents, related to the injection of the tissue adhesives, and the risk of damage to the instrument.

Uncontrolled studies have shown that thrombin is effective in controlling acute haemorrhage, but its role would probably be in the treatment of bleeding gastric varices for which the conventional sclerosants 
are inappropriate (Williams et.al. 1994). Larger studies are needed to establish its role.

\section{Balloon Tamponade}

This form of treatment is highly effective and controls acute bleeding in up to $90 \%$ patients, although about $50 \%$ of patients re-bleed when the balloon Is deflated (Panes et al 1988). It is, however, associated with serious complications such as oesophageal ulceration and aspiration pneumonia in up to $15-20 \%$ patients. Despite this, it is a life saving treatment in cases of massive uncontrolled variceal haemorrhage pending other forms of treatment.

\section{Liver Transplantation}

This is probably only appropriate for the patients who bleed while awaiting liver transplantation. Studies comparing variceal band ligation or transjugular intrahepatic portal-systemic shunt with liver transplantation is difficult to compare because it is an exceedingly uncommon option for the vast majority of patients, both because it is not commonly available and due to shortages and delays in organ procurement. No controlled trials of liver transplantation in uncontrolled/active bleeding are available.

\subsection{Risk of recurrent bleeding}

Rates of rebleeding vary from $60 \%-90 \%$ depending firstly, upon the number of patients in these studies with Child class $\mathrm{C}$ disease and secondly, upon the duration of follow up. Looking at the control groups of the propranolol trials for the secondary prevention (Lebrec et.al. 1984, Burroughs et.al. 1983, Villeneuve et.al. 1986, Colombo et.al. 1989, Garden et.al. 1990) of

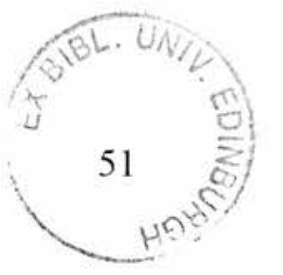


variceal rebleeding the highest risk of rebleeding was found by Garden et.al. and Villeneuve et.al. who found rebleeding in $70 \%$ and $75 \%$ patients respectively. They had included $46 \%$ and $30 \%$ patients with Child class C liver disease respectively. In contrast, rebleeding was reported in only $51 \%$ and $48 \%$ in studies by Quieniet et.al (Quieniet et.al. 1987) and Colombo et.al. (Colombo et.al. 1989) who studied only patients with Child class A and B disease or $96 \%$ were Child class A or B patients respectively.

\subsection{Secondary prophylaxis of variceal haemorrhage}

This form of treatment is aimed to prevent recurrence of variceal bleeding.

Surgery

Portacaval shunts may either be non-selective or selective. Nonselective shunts (eg portacaval shunt, PCS) refer to virtually total diversion of portal blood flow into the systemic circulation, thereby reducing liver blood flow. Selective shunts (eg distal spleno renal shunt, DSRS) refer to drainage of the varices into the systemic circulation without affecting the portal blood flow.

Four trials have compared non-selective shunts with no active treatment (Jackson et.al. 1968, Resnick et.al. 1974, Rueff et.al. 1976, Reynolds et.al. 1981) and all of these have shown a significant reduction in rebleeding, and mortality was reduced in 3 (Jackson et.al. 1968, Resnick et.al. 1974, 1976, Reynolds et.al. 1981) and increased in 1 (Rueff et.al. 1976). This difference was thought to be related to the randomisation procedure. 
Six trials compared non-selective shunts with the distal splenorenal shunt (DSRS) (Reichle et.al. 1979, Fischer et.al. 1981, Langer et.al. 1985, Millikan et.al. 1985, Harley et.al. 1986, Grace et.al. 1988) and included a total of 336 patients. No difference in rebleeding or encephalopathy was observed in any of the studies. Mortality was not significantly different in 5 of the trials, although it was significantly reduced in 1 trial (Fischer et.al. 1981).

DSRS has been compared with sclerotherapy in 4 randomised trials (Rikkers et.al. 1987, Teres et.al. 1987a, Henderson et.al. 1990, Spina et.al. 1990) and with PCS (Cello et.al. 1987, Korula et.al. 1988, Planas et.al. 1991) in 3 trials. Shunt surgery was associated with a significantly lower rebleeding in 5 of the trials (Rikkers et.al. 1987, Teres et.al. 1987a, Henderson et.al. 1990, Spina et.al. 1990, Planas et.al. 1991) and also in a meta-analysis (OR $0.18,95 \% \mathrm{Cl} 0.12-0.28)$. Incidence of $\mathrm{HE}$ after shunt surgery was also significantly greater than sclerotherapy in 4 studies (Rikkers et.al. 1987, Teres et.al. 1987a, Henderson et.al. 1990, Planas et.al. 1991) and remained unchanged in two (Cello et.al. 1987, Spina et.al. 1990). Mortality was increased significantly in the PCS group in 1 study but overall there were no significant differences.

$\beta$ blockers

A total of 755 patients were randomised in 11 trials comparing propranolol or nadolol (Gatta et.al. 1987) with no active treatment (Burroughs et.al. 1983, Lebrec et.al. 1984, Villeneuve et.al. 1986, Queuniet et.al. 1987, Gatta et.al. 1987, Colombo et.al. 1989, Sheen et.al. 1989, Garden et.al. 1990, Rossi et.al. 1991, Cerbelaud et.al. 1986, Colman et.al. 1990). 
Significant reduction in rebleeding was seen in 4 trials and a meta-analysis showed significant overall reduction ( $\mathrm{OR} 0.495 \% \mathrm{Cl} 0.3-0.54)$. Eight trials showed significant reduction in mortality, as did the overall analysis.

\section{Sclerotherapy}

One thousand one hundred and eleven patients have been randomised to either sclerotherapy or no active treatment in 8 trials (Rossi et.al. 1991, Terblanche et.al. 1983, CEVSP 1984, Westaby et.al. 1984, Soderlund et.al. 1985, Korula et.al. 1985, Burroughs et.al. 1989, Gregory et.al. 1991). Rebleeding was significantly reduced in 2 studies (Westaby et.al. 1984, Korula et.al. 1985). Overall there was also a significant reduction in rebleeding (OR $0.6395 \% \mathrm{Cl} 0.49-0.79)$. Mortality was significantly reduced in 1 study (Westaby et.al. 1985). Overall there was a significant reduction in mortality (OR $0.7795 \% \mathrm{Cl} 0.61-0.98)$.

Sclerotherapy has been compared with $\beta$ blockers in 9 trials which have randomised a total of 787 patients (Rossi et.al. 1991, Alexandrino et.al. 1988, Dollet et.al. 1988, Westaby et.al. 1990, Martin et.al. 1991, Dasarathy et.al. 1992, Fleig et.al. 1987, Liu et.al. 1990, Teres et.al. 1993). Significant reduction in rebleeding was found in the sclerotherapy group in 2 studies (Dasarathy et.al. Liu et.al. 1990) and increase was noted in 3 studies (Rossi et.al. 1991, Dollet et.al. 1988, Martin et.al. 1991) which was not statistically significant. The rest of the studies showed a reduction in rebleeding which was not statistically significant. The overall results were significantly heterogeneous and therefore a meta-analysis is not strictly valid which 
showed significantly reduced rebleeding with sclerotherapy. No significant differences were detected in mortality.

No significant differences were detected in a meta-analysis of 10 trials comparing sclerotherapy with sclerotherapy and $\beta$-blockers (D'Amico et.al. 1995). Two trials comparing sclerotherapy and $\beta$-blockers with $\beta$-blockers showed that the combined therapy reduced rebleeding and mortality significantly.

There are now 7 fully published trials in the literature comparing sclerotherapy with variceal band ligation (VBL) which were combined in a meta-analysis (Steigmann and Goff 1992, Gimson et.al. 1993, Laine et.al. 1993, Lo et.al. 1995, Hou et.al. 1995, Hashizume et.al. 1993, Laine et.al. 1996) This included 547 patients and concluded that variceal band ligation carried a significantly lower rate of rebleeding (OR $0.5295 \% \mathrm{Cl} 0.37-0.74)$, mortality (OR $0.6795 \% \mathrm{Cl} \quad 0.46-, 0.98$ ) and complications such as oesophageal stricture (OR $0.1095 \% \mathrm{Cl}$ 0.03-0.29).

These studies comparing the two best available modalities of treatment have shown that VBL reduces rebleeding, mortality and local complications. VBL should now considered as the endoscopic treatment of choice for patients with oesophageal variceal bleeding.

\subsection{Pitfalls in the assessment of prognosis}

There is inherent bias in assessing the natural history and the risk of first variceal bleed when exploring the relationship between the time of development of varices, the time of diagnosis and the time when the patient is recruited into the study. It is therefore logical that any assessment must be 
fundamentally flawed because of inclusion into these studies of patients who have been known to have varices for different lengths of time prior to inclusion into the study. These patients may have different natural histories, i.e. 'bleeders' or 'not bleeders'.

Smith and Graham (1981) showed that the time interval between admission to the hospital and the entry into clinical studies of variceal bleeding could influence the results of the study quite dramatically. This observation has been confirmed in a study by Burroughs et.al. (Burroughs et.al. 1989) who showed that when assessing rebleeding, if the starting point was moved from 0-5 days the proportion free of rebleeding at 25 days changed dramatically $(25-41 \%, p<0.001)$. Mortality is similarly affected. This is significantly more marked when assessing 30 day or 6 week mortality than when assessing 1 year mortality. Delaying the starting point of analysis tends to select out patients who are poor-risk.

\subsection{SODIUM RETENTION, ASCITES AND THE HEPATORENAL}

\section{FAILURE}

Ascites in patients with cirrhosis is a major cause of morbidity and a serious prognostic development. The kidney is central to the development of ascites and the purpose of this section is to discuss the pathogenesis and the principles of management of the renal dysfunction of cirrhosis. There is a spectrum of renal abnormalities in cirrhosis. In its mildest form, there is retention of sodium and the development of diuretic responsive ascites, 
which may progress to diuretic refractory ascites. The most severe form is manifested as the hepatorenal syndrome (HRS).

Refractory ascites is defined as ascites that cannot be mobilised or its early recurrence (after therapeutic paracentesis) cannot be prevented by medical therapy. The term includes diuretic-resistant ascites in which there is a lack of response to dietary sodium restriction and intensive diuretic treatment, and diuretic-intractable ascites where there is development of diuretic-induced complications that precludes the use of an effective diuretic regimen (Arroyo et.al. 1996). The HRS can be classified into type 1, which is characterised by rapidly progressive reduction of renal function defined by a doubling of the initial serum creatinine to a level greater than $2.5 \mathrm{mg} / \mathrm{dl}(250 \mu \mathrm{mol} / \mathrm{l})$ or a $50 \%$ reduction of the initial 24-hour creatinine clearance to a level lower than $20 \mathrm{ml} / \mathrm{min}$ in less than 2 weeks, and type 2 in which the renal failure does not have such a rapidly progressive course (Arroyo et.al. 1996). The kidneys are histologically normal in HRS.

\subsection{PATHOGENESIS}

The pathogenesis of renal dysfunction in cirrhosis is controversial and incompletely understood but can be thought of as having an afferent and an efferent component (Figure 1.1). Patients with cirrhosis exhibit characteristic circulatory abnormalities which are manifested by an increase in cardiac output, arterial hypotension, reduced peripheral vascular resistance and splanchnic vasodilatation. The pathogenesis of these circulatory 
Fig I.I Schematic representation of factors involved in the initiation and maintenance of sodium retention in patients with cirrhosis

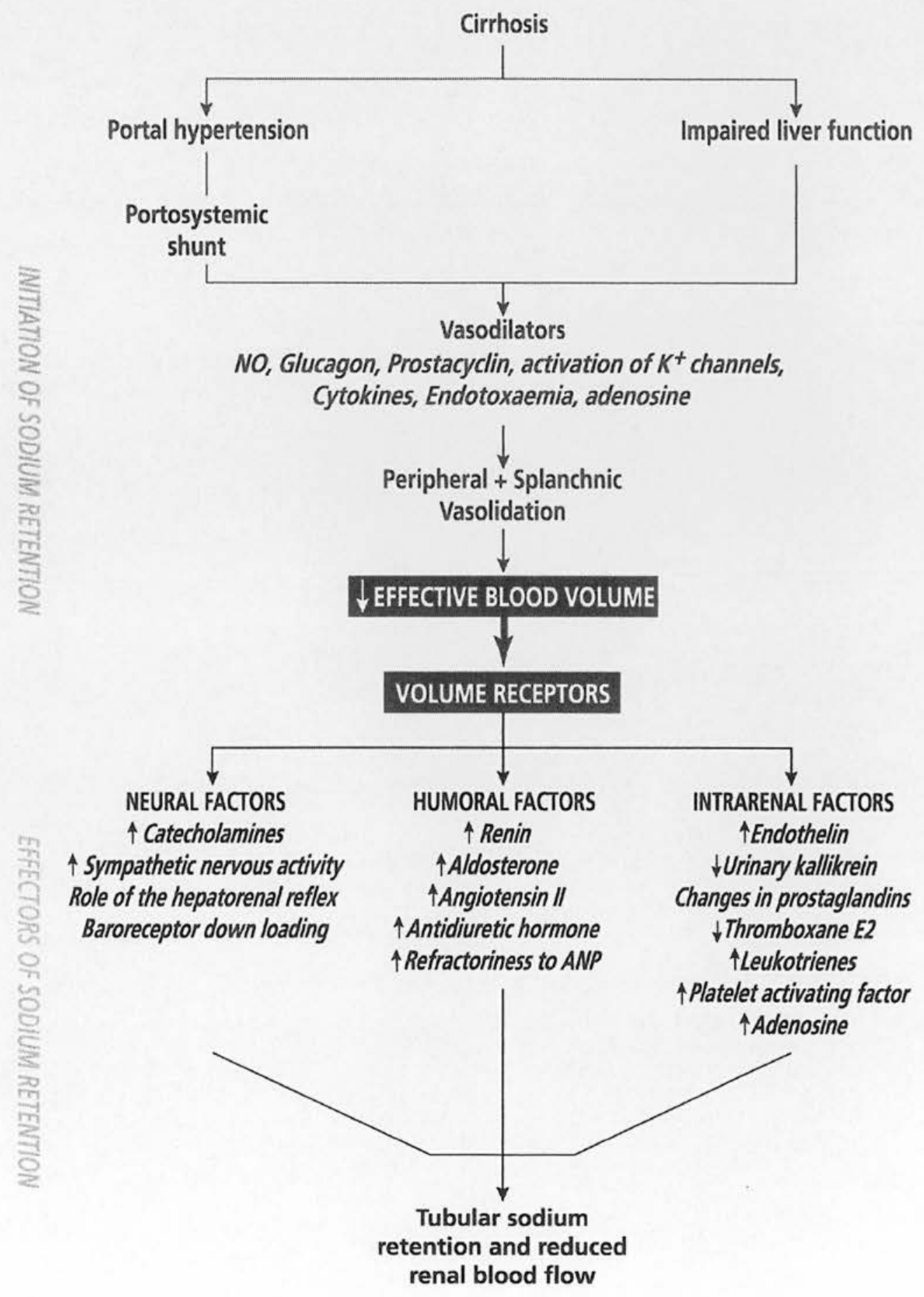


abnormalities are not clear but seems to be due either to portosystemic shunting and/or impaired clearance of vasodilators such as nitric oxide, endotoxins, prostacyclin, glucagon and adenosine. These vasodilators cause peripheral and splanchnic vasodilatation, which is perceived as a reduction in 'effective' plasma volume. This leads to the activation of neurohumoral factors which attempt to maintain the arterial pressure. These changes in the neurohumoral systems and activation of intrarenal factors is responsible for the renal dysfunction of cirrhosis (Schrier et.al. 1988). In addition, there appears to be a direct relationship between the severity of hepatic dysfunction and the onset of renal manifestations which argues for an interaction between the liver and the kidneys which has been postulated to be neural or biochemical in nature (Wong et.al. 1997, Jalan et.al. 1997). Localisation of fluid to the abdomen is the result of the abnormal Starling's forces in this area.

Patients with cirrhosis and portal hypertension manifest renal dysfunction with sodium retention, water retention and HRS. These manifestations may either present de novo or appear step wise. The following section deals with the pathogenic mechanisms associated with these syndromes.

\subsection{Retention of Sodium}

There is considerable evidence for increased activity of the reninangiotensin-aldosterone system (RAAS) (Bosch et.al. 1980, Hayes et.al. 1992). High levels of aldosterone, plasma renin activity and angiotensin II 
have been demonstrated in patients with cirrhosis which is probably due to increased production rather than reduced metabolism of these substances by the liver. Plasma renin activity is inversely related to sodium excretion and to renal blood flow. Concentrations of angiotensin II are also significantly higher in cirrhotics with ascites than those without (Tobe et.al. 1993). Atrial natriuretic factor is a powerful vasodilator and its role in sodium retention remains controversial. Concentrations of the hormone are raised in patients with cirrhosis but its effects are not pronounced because of marked renal refractoriness. This refractoriness may enhance the action of the vasopressors (Gines et.al. 1988a). Several studies have shown that plasma norepinephrine levels are elevated in patients with cirrhosis who have ascites and this is because of increased production rather than reduced clearance (Ring-Larsen 1982). Renal blood flow has been shown to be inversely related to norepinephrine levels. These data suggest that the activity of the sympathetic nervous system is enhanced and plays a significant role in sodium retention in cirrhosis (Henriksen et.al. 1984). Urinary kallikrein excretion in patients with cirrhosis and ascites deteriorates in parallel with the glomerular filtration rate. Infusion of aprotinin (kallikrein inhibitor) produces marked improvement in renal blood flow, glomerular filtration rate and sodium excretion (MacGilchrist et.al. 1994). The role of prostaglandins in the renal dysfunction of cirrhosis is not clear. Urinary excretion of its metabolites is increased when ascites is present but reduced in some patients with HRS (Moore et.al. 1990). Cyclo-oxygenase inhibitors such as non-steroidal antinflammatory drugs increase renal vascular resistance and reduce 
glomerular filtration rate in patients without ascites. These agents can produce oliguric renal failure in patients with ascites (Gines et.al. 1986). Endothelin is the most potent naturally occurring vasoconstrictor substance known. Elevated levels of endothelin-1 have been found in patients with ascites. This does not change either with volume expansion or tilting, and no correlation has been observed with endotoxaemia. The exact physiological role of this elevation is still not clear (Gerbes et.al. 1995, Rockey D 1997).

\subsection{Retention of Water}

Hyponatremia is the biochemical expression of reduced water excretion. In patients with cirrhosis it may occur either due to diuretics or spontaneously. The former is diagnosed by low central filling pressures. Patients with cirrhosis exhibit an inverse relationship between the levels of antidiuretic hormone $(\mathrm{ADH})$ and glomerular filtration rate. The use of a Kappa opioid receptor agonist, RU51599, which inhibits anti diuretic hormone (ADH) secretion enhances free water clearance (Moreau et.al. 1996). Similarly, demeclocycline, which is an $\mathrm{ADH}$ antagonist has been shown to improve free water clearance (De Troyer et.al. 1976). Patients with ascites and cirrhosis have diminished glomerular filtration rate which may reduce the delivery of filtrate to the diluting segments thereby reducing water clearance (Schedl HP and Bartter FC 1960). Increasing the distal delivery of filtrate using plasma volume expansion improves free water generation. Renal prostaglandins increase free water generation by numerous mechanisms. Although the data do not clearly establish a role for the renal prostaglandins, the relative 
deficiency of renal prostaglandins may contribute to the pathogenesis of water retention (Epstein M 1985a). Data regarding the role of the sympathetic system in causing water retention is not entirely clear. Studies have shown an inverse relationship between the plasma concentrations of norepinephrine and free water clearance but maneuvers which reduce sympathetic stimulation demonstrate no relationship between changes in free water clearance and plasma norepinephrine (Epstein et.al. 1985b).

\subsection{Hepatorenal syndrome}

The HRS is characterised by severe renal hypoperfusion due to an increase in renal vascular resistance. It develops in the setting of decompensated liver disease, poorly controlled ascites, and marked alterations in splanchnic and systemic haemodynamics. In some patients, HRS may be precipitated by gastrointestinal bleeding, sepsis, use of non-steroidal or nephrotoxic agents, hypovolemia, progressive liver failure or excessive use of diuretics (Epstein et.al. 1985b). The pathogenesis of the HRS is multifactorial. The sympathetic nervous system is highly activated and this produces renal vasoconstriction and reduction in renal plasma flow (Henriksen $\mathrm{JH}$ and RingLarsen $H$, 1994). Poor liver function and altered splanchnic haemodynamics further contribute to this renal vasoconstriction through the hepatorenal axis. RAAS is stimulated in cirrhosis and produces afferent arteriolar vasoconstriction (Jalan et.al. 1997, Lang et.al. 1991). Further renal vasoconstriction is produced by increased endothelin-1, the concentration of which correlates with the severity of renal dysfunction. Renal prostaglandins 
usually compensate for the vasoconstriction induced by the various vasopressors (Moore et.al. 1991). In HRS, this compensatory mechanism fails to maintain renal perfusion. Patients with HRS demonstrate a decrease in the mean arterial pressure which is a risk factor for its development (Gines et.al. 1993). In normal circumstances, the renal blood flow is autoregulated but in patients with HRS who have an activated neurohumoral system the autoregulatory curve (renal blood flow/renal perfusion pressure) is shifted to the right (Persson et.al. 1990). This means that a small reduction in the arterial pressure produces pronounced reduction in renal blood flow.

\subsection{PRINCIPLES OF MANAGEMENT}

Ascites is a frequent complication of cirrhosis and about $50 \%$ patients develop this complication within 10 years of the diagnosis of cirrhosis. Of the patients who have ascites, $18 \%$ develop HRS at 1 year and $39 \%$ at 5 years (Gines et.al. 1993). A patient with ascites may present with moderate ascites, tense ascites, refractory ascites, hyponatremia or with HRS.

The principles of management are summarised in Figure 1.2. Restriction of dietary sodium (to $80 \mathrm{mmol} / \mathrm{day}$ ) and use of diuretics are the most important modalities of treating ascites. When ascites is diagnosed, it is important to admit the patients to the hospital to establish the underlying diagnosis, exclude spontaneous bacterial peritonitis and educate the patient regarding their diet which will remain the corner stone of treatment. In patients with moderate ascites, the use of a combination of spironolactone and frusemide 
and dietary sodium restriction is the most effective regimen for controlling ascites, reducing hospitalisation and hypokalaemia. Patients should be monitored using their body weight, and the dose of the diuretics adjusted to produce a weight loss of $1 \mathrm{Kg}$ per day in patients with ascites and peripheral oedema and $500 \mathrm{gm}$ in patients with ascites alone. Total paracentesis with infusion of human albumin solution ( 8 gram per litre of ascites drained) is recommended for patients who present with tense ascites. When they are discharged from the hospital they should remain on dietary sodium restriction and diuretic therapy as discussed above.

The options for the management of refractory ascites are total paracentesis, peritoneovenous shunt, TIPSS and orthotopic liver transplantation. The most important advance in the management of refractory ascites has been the reintroduction of large volume paracentesis in the management of ascites. This method of treatment is both safe and effective as long as adequate plasma volume expansion with human albumin solution is provided (Gines et.al. 1988). A cheaper alternative is to use Dextran 70 and other synthetic gelatins which is effective but may be followed by subtle circulatory disturbances (Planas et.al. 1990). Insertion of TIPSS reduces portal pressure and has been suggested as a treatment for refractory ascites. Although some authorities suggest that this is useful in the treatment of refractory ascites (Ochs et.al. 1995) a randomised trial comparing TIPSS with paracentesis showed reduced survival in the patients treated with TIPSS (Lebrec et.al. 1996). At present, TIPSS should be used for refractory ascites 
as a part of randomised trials only. Peritoneovenous shunts are based on the principle of a pressure difference between the intraabdominal pressure and the superior vena cava in the thorax. A randomised study comparing the LeVeen shunt with paracentesis showed that paracentesis was as effective as LeVeen shunt in relieving refractory ascites, but the shunts were associated with better long-term control of ascites, shunt occlusion was common (50\%) and there were no significant differences in survival between the groups (50\%, at 1 year in both groups) (Gines et.al. 1991). Patients with refractory ascites who are otherwise good candidates for transplantation should undergo liver transplantation as this treatment improves survival significantly.

The presence of hyponatremia in a patient with ascites denotes a dilutional state and an impaired capacity to excrete water. Therefore, the most important modality to treat this complication is adequate water restriction. Although demeclocyline has been suggested by some investigators, this form of treatment should be avoided in patients with cirrhosis for the fear of precipitating renal failure (De Troyer et.al. 1976). Some patients may benefit from plasma volume expansion which acts by increasing the delivery of fluid to the diluting segments of the kidney (McCormick et.al. 1990). The effect of over-diuresis must be considered when ascites and oedema is absent and ruled out by the measurement of central venous filling pressure. The effect of nephrotoxic drugs is excluded on the history. 
The mortality in patients with the HRS and end stage cirrhosis approaches $100 \%$ (Gines et.al. 1993). Management of HRS is largely supportive and care must be taken to remove potential precipitating factors such as hypovolemia, drugs and sepsis. Although TIPSS may improve renal function (Brensing et.al. 1997) it has not been shown to reduce mortality and can at present be regarded as no more than a bridge to orthotopic liver transplantation which is the only form of treatment that reduces mortality in patients with refractory ascites and HRS (Moore KP, 1997, Forrest et.al. 1996).

The first abnormality leading to sodium and water retention in cirrhosis is the renal tubular defect that is related to the deteriorating liver function and hyperaldosteronism. With progression of liver disease and portal hypertension renal blood flow falls because of the hepatorenal reflex, and is then maintained by the vasoactive hormonal systems. With increasing peripheral vasodilatation, intrarenal factors that attempt to maintain renal perfusion cause intense cortical vasoconstriction. The systemic vasoactive factors are predominantly compensatory; any attempts to counteract their action risk circulatory collapse. Future studies should be directed at intrarenal factors. The ideal drug for the treatment of portal hypertension would reduce portal pressure, increase renal blood flow and produce insignificant changes in arterial pressure.

\subsection{Natural History And Prognosis Of Patients With Ascites}




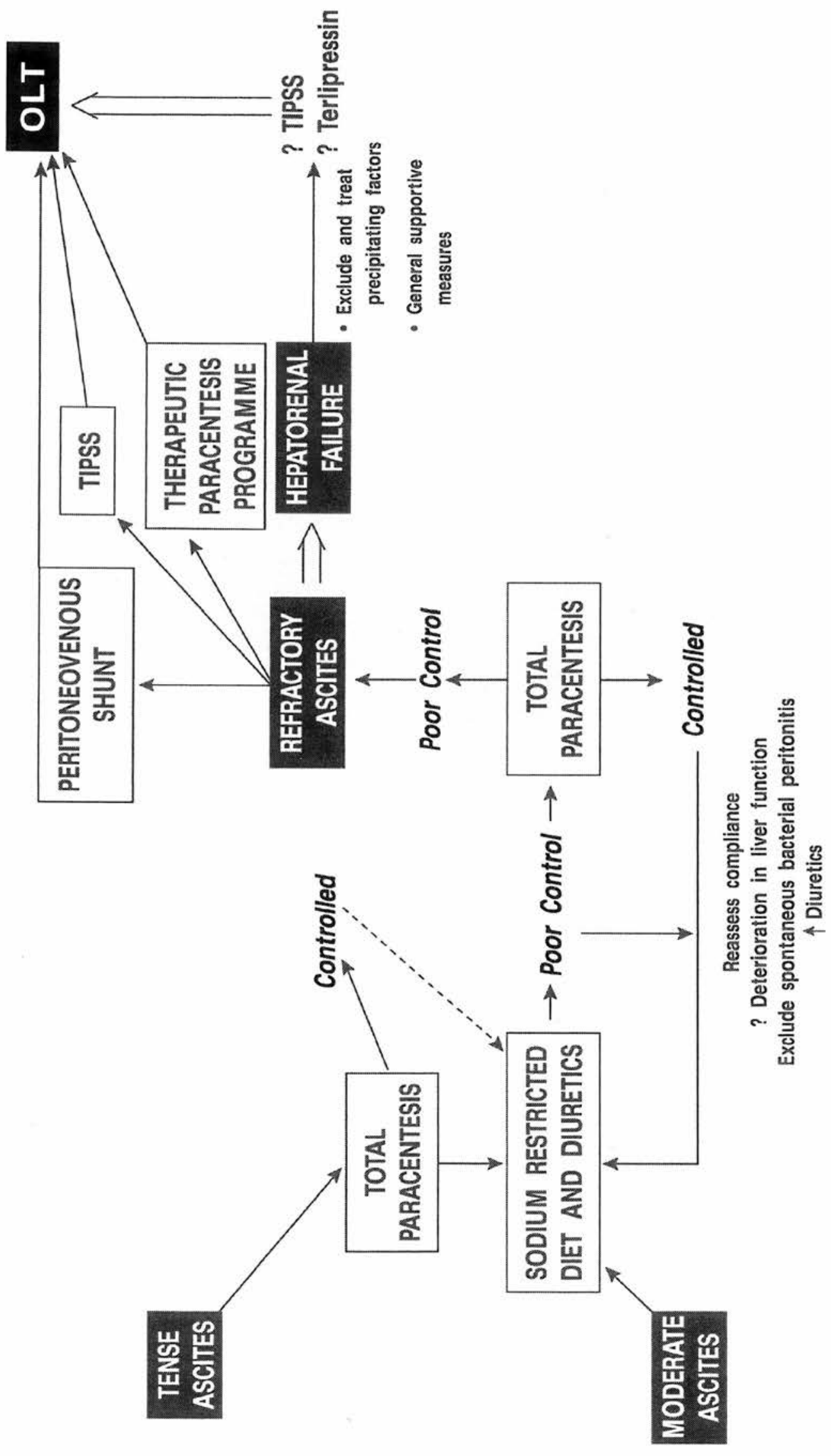


The appearance of ascites in cirrhosis carries a poor prognosis and the probability of one and five years survival after the first episode of ascites has been estimated at 15 and $20 \%$ respectively (Arroyo et al 1996). Arroyo also showed that patients with cirrhosis and renal failure die within weeks or months independent of the degree of hepatic insufficiency. Arroyo et al (1996) and Genoud et al (1986), performed two large multivariate analyses and assessed prognostic factors responsible for death in patients with cirrhosis. Llach et al (1988) studied 38 variables in 139 patients suffering from ascites in cirrhosis and showed that mean arterial pressure, plasma noradrenaline concentration, glomerular filtration rate, urinary sodium excretion, nutritional status, hepatomegaly and serum albumin concentration independently predicted mortality. Tage-Jensen et al (1988) showed in a multivariate analysis in 81 alcoholic cirrhotics that the presence of ascites, plasma noradrenaline concentration, portal pressure and serum bilirubin concentration independently predicted mortality. Both these studies indicate that the parameters such as systemic and portal haemodynamics, and renal function predicts survival better than the estimated hepatic function in patients with cirrhosis and ascites.

\subsection{HEPATIC ENCEPHALOPATHY}

Hepatic Encephalopathy (HE) is a neuropsychiatric syndrome with potential for full reversibility which occurs in patients with significant liver dysfunction and cannot be attributable to other causes. Two distinct forms can be identified in patients with cirrhosis. The 'overt' form which is easy to diagnose 
and the 'subclinical' form (SHE). The prevalence of SHE in cirrhosis varies from $30-84 \%$, and this wide variation is due to differences in definition, diagnostic methods and patients studied. It is defined as abnormalities in electrophysiological and psychometric tests and the most clinically useful tests for its diagnosis are the number connection test, digit symbol test and the digit copying test. The role of electroencephalography is diagnostically limited. Because the level of consciousness is not disturbed, its clinical impact is difficult to judge. Its presence has, however, been reported to be linked with a reduced ability to drive and with poorer quality of life (Quero JC, Schalm SW, 1996). Overt HE is associated with asterixis, hyper-reflexia, and with an electroencephalography showing slowing of the dominant rhythm. Lateralising neurological signs should not be attributed to HE without further investigations such as CT scan of the brain. Various methods have been described for estimating the severity of $\mathrm{HE}$ but the one that is clinically most useful is the West-Haven criteria (Conn HO and Leiberthal MM, 1979). However, this method has limitations because it is subjective, and has little relationship with prognosis. A more objective system of classification is being currently developed. This section will focus upon recent developments in the pathogenesis and the principles of management of hepatic encephalopathy that occurs in cirrhosis.

\subsection{PATHOGENESIS}




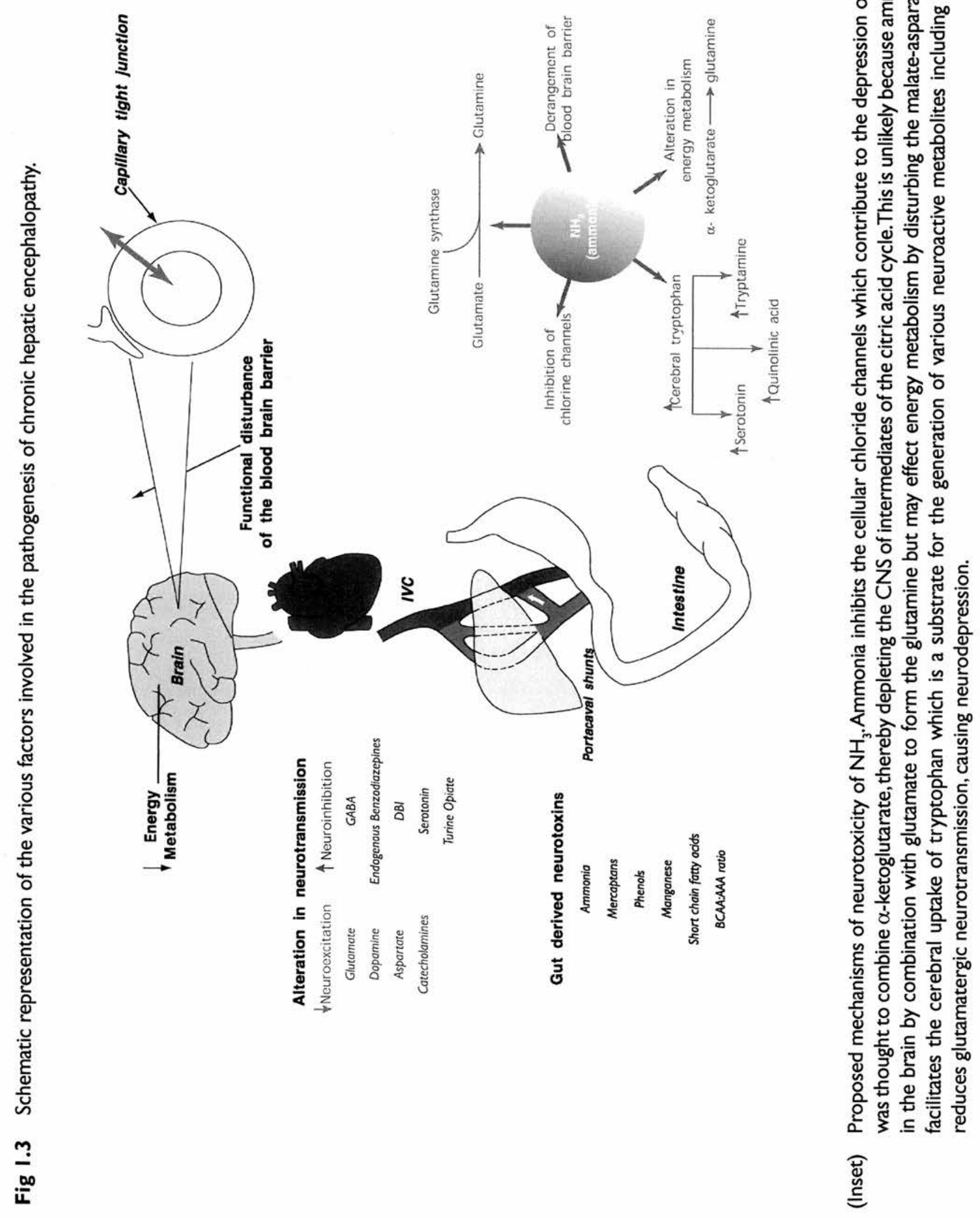


The pathogenesis of $\mathrm{HE}$ remains unclear and many different mechanisms have enjoyed periods of popularity. The most important factors are summarised in Figure 3.3.

\subsection{The role of alterations in the blood brain barrier (BBB)}

The BBB ensures protection of the biochemical environment of the brain. Patients with $\mathrm{HE}$ exhibit a functional derangement in the BBB which results in increased transport of neutral amino acids and reduced transport of basic amino acids (Mans et.al. 1982). The mechanism of this functional disturbance is not clear although increased levels of ammonia and methanthiols have been implicated. Subtle indicators of disturbances in the BBB stem from studies using in vivo 31-phosphorus magnetic resonance spectroscopy (MRS, an in vivo method that can be used to quantify the concentration of brain metabolites) which show a reduction in choline in the brain in patients with $\mathrm{HE}$. It is likely that this disturbance in the BBB provides the mechanism whereby other factors in HE may operate (Kreis et.al. 1991).

\subsection{The role of changes in energy metabolism}

Patients with HE have reduced cerebral blood flow and glucose and oxygen consumption (Lockwood et.al. 1991). Using positron emission tomography scanning, alterations in cerebral blood flow have been shown to correlate with the severity of neuropsychological function (O'Carroll et.al. 1991). In animal models, abnormalities in cerebral energy metabolism are delayed until after the onset of coma (Hindfelt et.al. 1977). It is not easy to extrapolate this 
model to the human situation, but it is likely that changes in cerebral blood flow and glucose metabolism are an epiphenomenon, secondary to the global depression in CNS function, rather than the cause of $\mathrm{HE}$. Longitudinal studies are required for further clarification.

\subsection{The role of gut derived factors}

Any putative toxin that may be of pathogenic importance in HE should demonstrate the following characteristics. It should be (i) nitrogenous, (ii) originate in the gut, (iii) be produced by the action of gut bacteria or be present in the diet, (iv) be found in the portal circulation, (v) be metabolised in the liver and (vi) be able to cross the blood brain barrier (Basile et.al. 1963).

Ammonia: Historically, ammonia has been considered the most important factor in the genesis of $\mathrm{HE}$. In $\mathrm{HE}$, the concentrations of ammonia are elevated both in the systemic circulation and the cerebrospinal fluid. The proposed mechanisms of ammonia induced neurotoxicity are outlined in Figure 1. Factors supporting the importance of ammonia include hyperammonaemia caused by factors unassociated with cirrhosis or portosystemic shunting such as urea cycle enzyme deficiencies, which produce a syndrome resembling $\mathrm{HE}$, reduction of circulating ammonia levels by treatment with lactulose and antibiotics improving $\mathrm{HE}$, and the feeding of ammoniagenic substances precipitating encephalopathy in patients with cirrhosis. Observations which are against ammonia being the sole factor in the development of $\mathrm{HE}$ are the poor correlation between blood and 
cerebrospinal fluid ammonia concentration and the severity of $\mathrm{HE}$, the occurrence of HE despite normal levels of ammonia, and the seemingly contradictory effect of ammonia upon the CNS function with low ammonia concentrations producing CNS excitation rather than neurodepression (Basile et.al. 1991, Stahl J 1963). Recent studies focus upon the role of ammonia in the modulation of other neurotransmitter systems such as $\gamma$-aminobutyric acid, benzodiazepines and serotonin (Basile AS and Jones EA, 1997).

Other metabolites: Although derangements in mercaptans, phenols and fatty-acids have been described in $\mathrm{HE}$, there is no consistent evidence that they are of major importance in $\mathrm{HE}$ and possibly exert synergistic effects with ammonia. Proton $\left({ }^{1} \mathrm{H}\right)$ MRS studies of the brain in patients with $\mathrm{HE}$ show a reduction in the brain myo-inositol and an increase in brain glutamine (Lauenberger et.al 1996). In patients with HE, there is a good correlation between reduced myo-inositol and increased glutamine, with the severity of $\mathrm{HE}$. Derangements in plasma amino acid concentration in patients with $\mathrm{HE}$ include typically a pattern of increased aromatic amino acids and decreased or normal branched-chain amino acids (BCAA) (Basile et.al. 1991, Soeters et.al. 1977).

Manganese. Patients with cirrhosis have a characteristic abnormality in the $\mathrm{T}_{1}$ weighted magnetic resonance image (MRI) of brain which is manifested by hyperintensity of the globus pallidus (Mans et.al. 1982, Pomier-Layrargeus et.al. 1995). HE and manganese toxicity produce similar changes in MRI 
appearances of the basal ganglia. Increased manganese concentrations have been found in the basal ganglia of patients with $\mathrm{HE}$ and good correlation occurs between the MRI abnormalities and the blood manganese levels. Manganese levels may be elevated in patients with cirrhosis because of impaired excretion in bile but the significance of these findings are uncertain (Spahr et.al.1996).

\subsection{Changes in cerebral neurotransmission}

The normal state of wakefulness is determined by a fine balance between the state of excitatory and inhibitory neurotransmission. Changes in cerebral neurotransmission in $\mathrm{HE}$ are discussed below.

Glutamate is the most important cerebral excitatory neurotransmitter in the brain and its concentration at the synaptic level is dependent upon the glutamate-glutamine cycle whereby glutamate is released by the presynaptic neurons, reacts with the postsynaptic neurons, and is inactivated to glutamine in the astrocyte. In patients with $\mathrm{HE}$, cerebral glutamate is reduced (Butterworth RF 1994, Michalak et.al. 1996), reuptake mechanisms for glutamate are impaired and there is down regulation of glutamate binding sites on the post-synaptic neurons (Michalak et.al. 1996). All of these contribute to reduced neuroexcitation. This change is specific and correlates with the severity of $\mathrm{HE}$. 
inhibitors (DBI): GABA is an important neuroinhibitory neurotransmitter and although it does not cross the BBB, GABAergic tone is increased in $\mathrm{HE}$ (Basile et.al. 1991). Endogenous benzodiazepines are elevated in the plasma and CSF of patients with HE (Jones et.al. 1988). They may be present in the diet or may be metabolic products of the microbial flora of the gut (Mullen KD and Jones EA, 1996). Recent PET studies suggest a diffuse increase in the benzodiazepine receptor binding in the brain following administration of ${ }^{11}$ C-Flumazenil (Jalan et.al. 1996). DBI modulates the activity of GABAergic and glutamatergic tone by inducing the glial cells to produce neurosteroids which have been implicated in the pathogenesis of $\mathrm{HE}$ (Itzhak et.al. 1995). Its significance is unclear but all the above evidence suggests that an increased GABAergic tone may contribute to the neurodepression observed in $\mathrm{HE}$ (Lauenberger et.al. 1996, PomierLayrargeus et.al. 1995).

Catecholamines, dopamine, serotonin, 'false neurotransmission' and other metabolites Brain concentrations of catechecholamines are reduced in animal models of hepatic encephalopathy but this has not been substantiated in the humans. Dopamine ${ }_{2}$ receptors have been shown to be downregulated in patients with severe $\mathrm{HE}$ and may be the cause of the derangements in psychomotor function (Baraldi et.al. 1983). Serotonin concentrations are increased in animal models of $\mathrm{HE}$ but there is controversy regarding the concentration of cerebral serotonin receptors. The increased 
cerebral concentrations of aromatic amino acids inhibit tyrosine hydroxylase thereby generating 'false neurotransmitters' such as octopamine, tyramine and phenyl ethanolamine which are believed to compete with both catecholamine and dopaminergic neurotransmission. Although their concentration is increased in $\mathrm{HE}$, direct injection of octopamine into the brain of animals did not produce any significant abnormality (Fischer JE and Baldessarini RJ, 1971). Reduced aspartate (neuroexcitatory) has been found in comatose rats, levels of taurine (neuroinhibitory) are increased in animal models and opiate (neuroinhibitory) receptor density is increased in dogs with portacaval shunts (Basile et.al. 1991, Butterworth RF 1994).

$\mathrm{HE}$ appears to be a disorder of multiple neurotransmitter systems and ammonia plays a central role in its pathogenesis.

\subsection{PRINCIPLES OF MANAGEMENT}

The question regarding treatment of subclinical encephalopathy is unclear and this discussion will be limited to the principles of management of 'overt' HE. Patients with HE fall into two groups. First, are those patients who have episodes of encephalopathy and are relatively well between attacks. These patients usually have a precipitating event such as dietary protein loading, gastrointestinal bleeding, exacerbation of the underlying liver disease, sepsis, dehydration, hypokalemia, hypoxia, use of sedatives, and constipation. Second, are patients with spontaneous encephalopathy. Management of HE therefore involves the detection and treatment of the precipitating events, the 
treatment of $\mathrm{HE}$ itself and the treatment of the underlying liver disease. In patients with spontaneous $\mathrm{HE}$, measures should be introduced to prevent the occurrence of encephalopathy. Treatment efficacy is difficult to judge as about half of the patients with HE recover spontaneously. Therefore, large numbers of patients are required to assess the beneficial effect of a new treatment. Furthermore, many trials compare newer drugs against 'established treatments' which themselves have not been rigorously tested. A summary of different modalities of treatment are described in Table 1.1.

\section{Treatment aimed at reducing ammonia toxicity}

Management of the precipitating event, dietary protein restriction, avoidance of constipation and manipulation of the bowel flora remain the mainstays of therapy (Conn HO and Leiberthal MM, 1979, Ferenci et.al. 1996, Simmons et.al. 1970, Morgan MY and Hawkey KE 1987). Dietary protein restriction is effective but should only be used short-term to avoid deleterious nutritional consequences. Protein intake in individual patients requires careful titration. In three studies, vegetable diet was shown to be better tolerated than diets containing animal protein (Ferenci et.al. 1996). This was not confirmed in a further study, although the fiber content of the diet seems to have a favorable effect. In practice lactulose and lactitol are of greatest value in the short and in the long-term and they probably act by ensuring bowel movement, by affecting bacterial metabolism including ammonia production and absorption of ammonia. Antibiotics are rarely used nowadays. Neomycin is effective in modifying the bacterial flora but it has serious ototoxicity and renal toxicity, 
and should not be used long-term or where there is renal dysfunction. Alternative antibiotics, such as tetracyclin, metronidazole and vancomycin also have significant long term toxicity (Ferenci et.al. 1996). Newer agents such as sodium benzoate (Sushma et.al. 1992) and L-ornithine L-aspartate (Kircheis et.al. 1997) are aimed at metabolic removal of ammonia. Although both these agents have been studied in controlled clinical trials and shown to have significant beneficial effects in patients, they clearly require further studies before their role in clinical practice becomes defined.

\section{Treatment based on cerebral neurotransmission}

Five controlled trials of Flumazenil have been performed in patients with cirrhosis and varying severity of encephalopathy (Ferenci et.al 1996). Flumazenil was shown to be superior to placebo in 3 of the studies. In two studies, which included patients with mild HE or SHE, no significant improvement was demonstrated with Flumazenil. Overall, the use of Flumazenil in patients with HE cannot be routinely recommended apart from patients who have been administered benzodiazepines, and as a part of clinical studies.

\section{Other approaches}

Supplementation with BCAA was aimed at the 'false neurotransmitter hypothesis'. Of the nine controlled trials performed, only 3 have shown a beneficial effect of BCAA administration. This effect seems to be most beneficial in patients with $\mathrm{HE}$ who are severely intolerant of protein and no 
beneficial effects were observed in patients who were protein tolerant (Ferenci et.al. 1996, Fischer JE 1990). Orthotopic liver transplantation is the only treatment which reverses the mental disorder permanently by correcting both the poor liver function and the portacaval shunting. Other treatments under current investigation include drugs aimed at serotonin, opiates, melatonin and zinc metabolism.

$\mathrm{HE}$ is a complex metabolic disorder which may result from regional rather than global effects in the brain. No single mediator has been found to account for the syndrome of $\mathrm{HE}$, and $\mathrm{HE}$ may result from the effect of several interacting mediators. Further research should be directed at developing newer radioligands for PET and increasing the sensitivity of the MR spectroscopy which will provide a better understanding of the biochemical and metabolic changes directly. Effective therapy will only emerge once the pathogenesis of this syndrome is fully understood. 


\section{Studies}

Controlled

\begin{tabular}{lll}
\hline Aim of treatment & $\begin{array}{l}\text { vs } \\
\text { lactulose }\end{array}$ & $\begin{array}{l}\text { vs } \\
\text { placebo }\end{array}$ \\
\hline AMMONIA HYPOTHESIS & & \\
$\begin{array}{l}\text { Reduce ammoniagenic substrate } \\
\text { Vegetable protein diet } \\
\text { Lactulose enema }\end{array}$ & \pm \\
$\begin{array}{l}\text { Inhibit production of ammonia } \\
\text { Neomycin } \\
\text { Vancomycin } \\
\text { Metronidazole } \\
\text { Lactobacillus }\end{array}$ & + \\
$\begin{array}{l}\text { Reduce absorption and production of ammonia } \\
\text { Lactulose }\end{array}$ & & $\mathrm{NA}$ \\
Lactitol & $=/+$ & $\mathrm{NA}$ \\
$\begin{array}{l}\text { Increase removal of ammonia } \\
\text { Ornithine-Aspartate } \\
\text { Sodium Benzoate }\end{array}$ & $=$ & NA \\
\end{tabular}

\section{GABA HYPOTHESIS}

Flumazenil

$\pm$

FALSE NEUROTRANSMITTER HYPOTHESIS
BCAA
L-DOPA, Bromocriptine
OTHERS
Zinc
Modified from Ferenci et.al.(1996)+ significantly better than control Rx, = as good as control
Rx, - no effect, \pm unclear data, NA-data not available.




\subsection{HYPERSPLENISM}

The cardinal features of hypersplenism are splenomegaly, reduction in one or more cellular elements of the blood and the presence of normal hypercellular bone marrow, and correction of blood cytopenias by splenectomy. This is not usually recommended as treatment in patients with cirrhosis. The incidence of hypersplenism in patients with cirrhosis varies between $36-90 \%$ in different series (Mutchnik et.al. 1980). The mechanism of splenomegaly in patients with cirrhosis which produces the congestive form of splenomegaly, is partly due to hyperplasia of the white pulp and fibrosis superimposed upon congestion of the red pulp (Michael et.al. 1934). There is a poor correlation between the splenic size and the portal pressure which suggests that passive congestion is not the sole mechanism involved in its development (Witte et.al. 1971). Another factor which may be important in its causation is lymphoid hyperplasia due to persistent stimulation induced by gut derived antigens which bypass the hepatic Kupffer cells (Rozga et.al. 1985). Nishida et.al. (Nishida et.al. 1990) have suggested that there may be a relationship between splenic and intestinal circulation with reciprocal changes in flow occurring in the two circulations to maintain portal venous pressure and flow. This suggests that the splenic circulation may play an important role in the maintenance of portal hypertension.

\subsection{HISTORICAL ASPECTS OF TRANSJUGULAR INTRAHEPATIC PORTOSYSTEMIC STENT-SHUNT (TIPSS)}


The idea of TIPSS emerged in 1969 when Rosch and Hanafee (1969) used the inadvertant punctures of the portal vein during transjugular cholangiography to develop the technique of transjugular portography. Following successful use of this technique for portography in experimental animals the next obvious logical step was to develop transjugular intrahepatic portosystemic shunt. Using a modified Ross needle they created a communication between the right hepatic vein and the right portal vein, dilated it with coaxial catheters and kept it open with non-expandable plastic or coilspring tubing of 4-6 mm in diameter (Rosch $\mathrm{J}$ and Hanfee WN, 1969, Rosch $\mathrm{J}$ and Hanafee WN, 1971). These shunts functioned for about 2 weeks before clotting. They concluded from these studies that TIPSS was a feasible technique but the technology available at the time prevented its application in humans.

In the 1970's further experiments were performed in animals using various methods for the creation an intrahepatic shunt. Various techniques, such as cutting, drilling and freezing using cryoprobes were used, each of which created a successful shunt but patency of the shunt was limited (Koch et.al. 1973, Reich et.al. 1977, Dotter et.al. 1981). The introduction of angioplasty balloons were instrumental in developing this technique further. Burgener and Gutierrez (Burgener FA and Gutierrez OH, 1979, Burgener FA and Gutierrez $\mathrm{OH}, 1988$ ) used balloon angioplasty in animals and demonstrated that they could keep the shunts patent for over a year with repeat balloon angioplasty. 
This was the impetus for the first clinical application of TIPSS which was performed by Colapinto (Colapinto et.al. 1982). The shunt was created using a $9 \mathrm{~mm}$ balloon which was kept inflated in the intraparenchymal tract for 12 hours. This resulted in a reduction in the portal pressure by $20 \mathrm{mmHg}$ and stopped variceal bleeding. They then expanded their experience and reported the use of TIPSS in 15 patients with cirrhosis and uncontrolled variceal haemorrhage. The mean reduction in the portal pressure following a successful TIPSS was $5.9 \mathrm{mmHg}$. This was also associated with immediate arrest of variceal haemorrhage (Gordon et.al.1987).

Palmaz was instrumental in the introduction of the expandable metal stent to 'cover' the intraparenchymal track (Palmaz et.al. 1985, Palmaz et.al. 1986). Their detailed experimental work on dogs showed clearly that a 10 $\mathrm{mm}$ diameter shunt normalised the portal pressure. Shunt function in dogs with portal hypertension remained normal for the full duration of the study which was 48 weeks. At autopsy they demonstrated that the shunt was endothelialised. Rosch et.al. (Rosch et.al. 1987) demonstrated the suitability of modified Gianturco stents for TIPSS in portal hypertensive pigs.

These experiments were the basis for the movement of TIPSS from the bench to clinical practice. The first clinical TIPSS, as we understand it today, i.e., using a metal stent was performed by Richter. He demonstrated that the portal pressure was reduced by $20 \mathrm{mmHg}$ and the patient improved clinically. This patient died 12 days after TIPSS due to adult respiratory distress syndrome and disseminated intravascular coagulation. The shunt was patent at autopsy. Since then numerous groups have reported their 
experience with the use of this technique in large numbers of patients. The groups which have provided the largest initial experience have been the Freiburg groups (Rossle et. al. 1994) in Europe and University of California, San Francisco in the United states (LaBerge et.al. 1993).

Although these studied have confirmed that TIPSS can be performed in large numbers of patients with high degree of success, numerous questions remain unanswered. The 'aims of this thesis' outline some of the current controversies and devises various experiments to try to answer them. 
AIMS OF THE THESIS 
The advent of TIPSS has provided a unique opportunity to access the portal venous system repeatedly, in patients with cirrhosis. This allows firstly, to study the role of portal pressure in the manifestations of portal hypertension in cirrhosis and provides an exciting opportunity to treat portal hypertension. Data about long-term follow up, its effects upon haemodynamics, liver function, neuropsychological function, haematology and its place in the management of complications of cirrhosis are still unknown. The purpose of this thesis was to answer four basic questions.

\section{What does TIPSS do to alter the natural history of cirrhosis?}

- Long term follow up

- Assess prognostic variables to predict mortality, rebleeding, encephalopathy and shunt insufficiency after TIPSS

- Study changes in Liver function after TIPSS

- Study changes in neuropsychological function after TIPSS

- Study haematological changes after TIPSS

2. What happens to shunt function after insertion and why does it occlude?

- Compare Doppler ultrasonography with portography in the assessment of shunt function after TIPSS

- Clinical, radiological and pathological study to define the pathogenic mechanisms in the development of shunt insufficiency

\section{What is the place of TIPSS in the management of portal hypertension} of cirrhosis?

- A retrospective study comparing TIPSS with oesophageal transection in the management of uncontrolled variceal haemorrhage 
- A randomised and controlled study comparing TIPSS with Variceal band ligation in the secondary prophylaxis of variceal bleeding

4. Can patients who have TIPSS be studied to understand the pathophysiology of portal hypertension better?

- Mechanism of changes in renal function after TIPSS

- Use of TIPSS in the evaluation of the 'hepatorenal reflex' 


\section{CHAPTER 2}

The Natural History, Manifestations and Prognosis Of TIPSS 
2.1 AIMS

This section was designed to answer the following questions

1. Prospectively evaluate the long term effects of insertion of Transjugular intrahepatic stent-shunt (TIPSS) to assess the natural history of the patient after TIPSS insertion and the effect upon shunt function during follow up.

2. To determine the prognostic indicators for mortality, variceal rebleeding shunt insufficiency and encephalopathy following TIPSS.

3. To prospectively assess changes in the neuropsychological state, measures of memory, quality of life and scores for anxiety and depression in patients without prior encephalopathy and their relationship with the change in portal pressure gradient.

4. To prospectively assess changes in biochemical tests of liver function and also in ICG clearance and their relationship with the changes in the portal pressure gradient.

5. To assess the changes in the red cell profile following the insertion of TIPSS with particular reference to haemolysis and secondly, to study changes in the spleen size and platelet counts and their relationship with changes in the portal pressure gradient. 


\subsection{METHODS}

\subsection{TIPSS insertion, assessment of shunt function}

\section{TIPSS Insertion}

All the TIPSS procedures were performed by a single interventional radiologist together with a hepatologist.

Operating environment and the operator TIPSS was performed in an angiography laboratory with facilities for high resolution C arm fluoroscopy, digital subtraction angiography and Doppler ultrasonography. Electrocardiogram, pulse rate, blood pressure and oxygen saturation were continuously monitored throughout the procedure. Access to resuscitative equipment was available throughout. The technique is essentially interventional radiological but close cooperation between a hepatologist and radiologist appears ideal. Other assistants who were available for the TIPSS procedure included a trained nurse and a radiographer.

Preparation for TIPSS Informed consent was obtained from all patients prior to TIPSS which when electively performed was usually the morning after an overnight fast. Prothrombin time was corrected to within 6 seconds of the normal range and platelet count maintained above 50,000/litre. We administered cefotaxime and augmentin routinely starting 1 hour prior to the procedure and continuing up to 48 hours afterwards because of the risk of sepsis. Midazolam and pethidine were used for sedation and analgesia 
during TIPSS. General anaesthetic was reserved for the severely agitated, actively bleeding patients (to protect the airway from the risk of aspiration) and those who could not tolerate sedation. No anticoagulants were used as a prophylactic measure against shunt thrombosis.

Pre procedure imaging Over the years our practice evolved from the routine transhepatic placement of a catheter into the portal vein to serve as a guide, to a superior mesenteric and coeliac angiogram (venous phase) to visualise the portal venous anatomy (first 32 patients), and to the use of duplex Doppler ultrasonography to mark the site of portal vein bifurcation (next 27 patients). In the rest of the patients, Doppler ultrasonography was performed prior to TIPSS only to rule out the possibility of portal vein thrombosis.

Type of stent There are a number of stents available but the two in common usage are the Palmaz (Johnson and Johnson, Interventional systems co., New Jersey, USA) and the Wallstents (Schneider, Ackerstrasse, Bulach, Switzerland). Both of these stents have theoretical advantages and disadvantages. Palmaz stents are short, rigid and more than one stent may be required for adequate shunt coverage. They do have the advantage of allowing graduated expansion to attain the portal pressure desired. The Wallstent is longer, more flexible, easier to deploy and more than 1 stent is seldom required. However, the stent diameter is fixed. The first 24 patients had Palmaz stents and all the rest have had Wallstents. 
TIPSS procedure The method of TIPSS insertion used was based upon the method described by Richter (1988) and modified by Rossle et.al. (1994). The right internal jugular vein was the common site of entry although the left internal jugular vein was used in about $10 \%$ patients. After sterile draping and dressing, the site of entry was infiltrated with $5-10 \mathrm{mls}$. of lignocaine. The internal jugular vein was punctured and, a $41 \mathrm{~cm}(10 \mathrm{~F})$ sheath (William-Cook, Sandet 6, Bjaeverskov, Denmark) was introduced over a guide wire into the inferior vena cava. The right or the middle hepatic vein was selected using a stiff hydrophilic guide wire (Terumo Corp. Hatagaya, Tokyo, Japan) and a 16 gauge, curved transjugular needle (William-Cook, Sandet 6, Bjaeverskov, Denmark) was introduced over the guide wire into the hepatic vein and used to guide a fine stylet anteriorly towards a branch of the portal vein. Several attempts were needed to puncture the portal vein and this was generally the most time consuming part of the procedure. Entry into the portal vein was associated with a marked resistance followed by a characteristic "give". Aspiration of blood followed by delineation of the portal system by injection of contrast medium indicated satisfactory entry. The hydrophilic guide wire was exchanged for an Amplatz wire (Medi-Tech, Boston scientific corporation, Watertown, Massachusetts, USA) and an angioplasty balloon catheter was used to dilate the parenchymal tract to an appropriate size to accommodate the stent to be used. This was determined by the age of the patient, the severity of portal hypertension, the indication for TIPSS and the presence or absence of encephalopathy. The aim was to reduce the portal pressure gradient to below $12 \mathrm{~mm} \mathrm{Hg}$ below which variceal bleeding. The dilatation of 
the tract was associated with appearance of 2 characteristic waists representing the hepatic and the portal veins respectively. This was usually the most painful part of the procedure and we used extra analgesia at this point. The balloon was then removed and either 1 or 2 Wallstents or 2-4 Palmaz stents inserted to support the tract open. This is depicted pictorially in Figure 2.1.

Pressure measurements and portography were performed at the time of portal vein catheterisation and after shunt creation to ensure adequate shunting. In occasional patients with uncontrolled variceal haemorrhage the varices were embolised using either steel coils or particulate matter such as spongostan.

\section{Evaluation Of Shunt Function During Follow Up}

Definition of shunt insufficiency The aim of TIPSS is to control variceal bleeding or prevent rebleeding by reducing the portal pressure gradient (PPG $=$ portal pressure - inferior vena cava pressure) to $<12 \mathrm{mmHg}$. This is considered by most authorities to be the critical figure below which variceal haemorrhage does not occur (Garcia-Tsao et.al. 1985). We therefore defined shunt insufficiency as an increase in the PPG to $>12 \mathrm{mmHg}$ in patients in whom the PPG was greater then $12 \mathrm{mmHg}$ at the time of insertion of TIPSS.

Timing and methods of evaluation of shunt function The timing of assessment of shunt function has varied some what during the study period. Initially, shunt function was assessed using Doppler ultrasonography prior to 

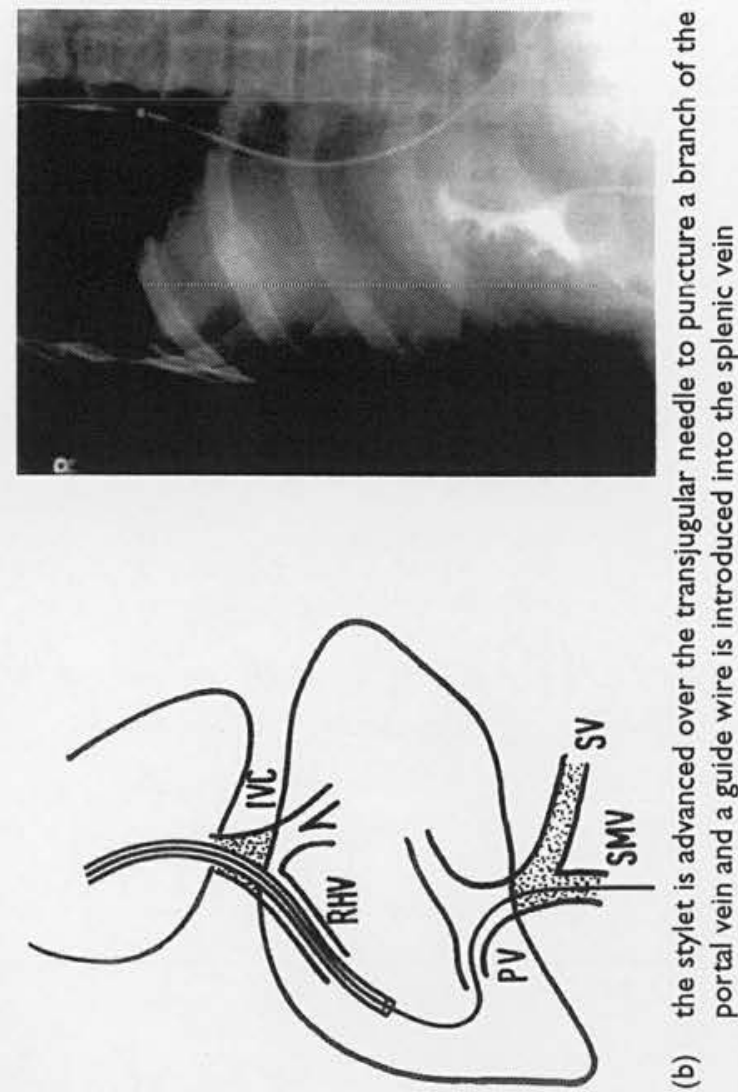

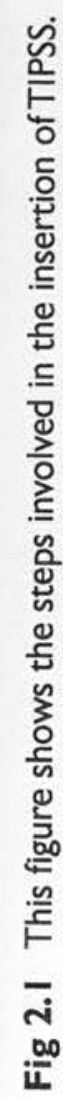

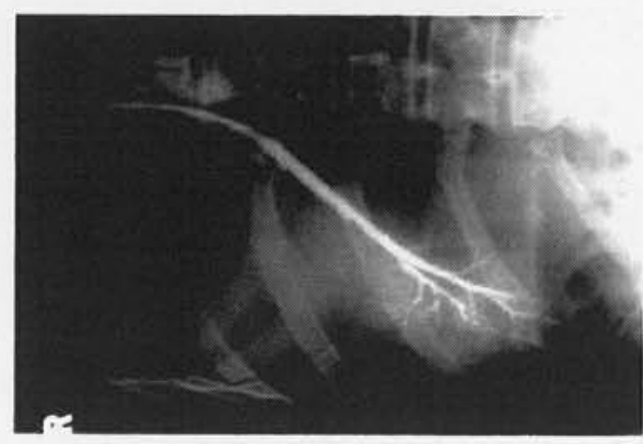

등
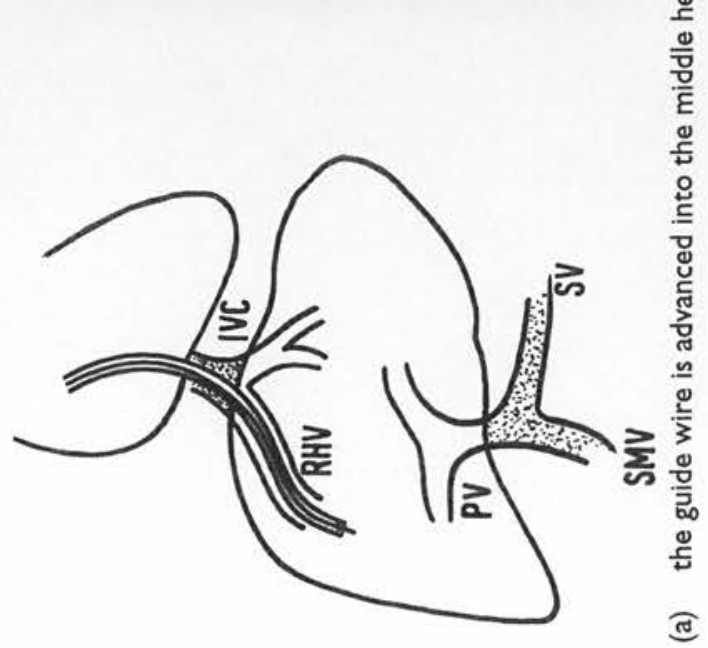
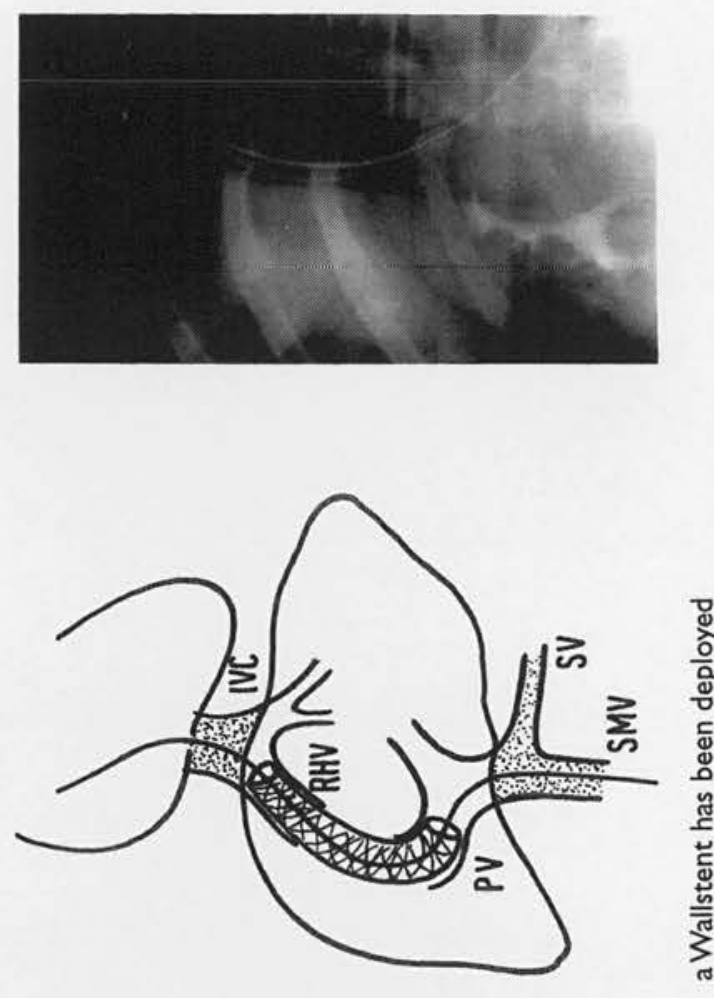

จ
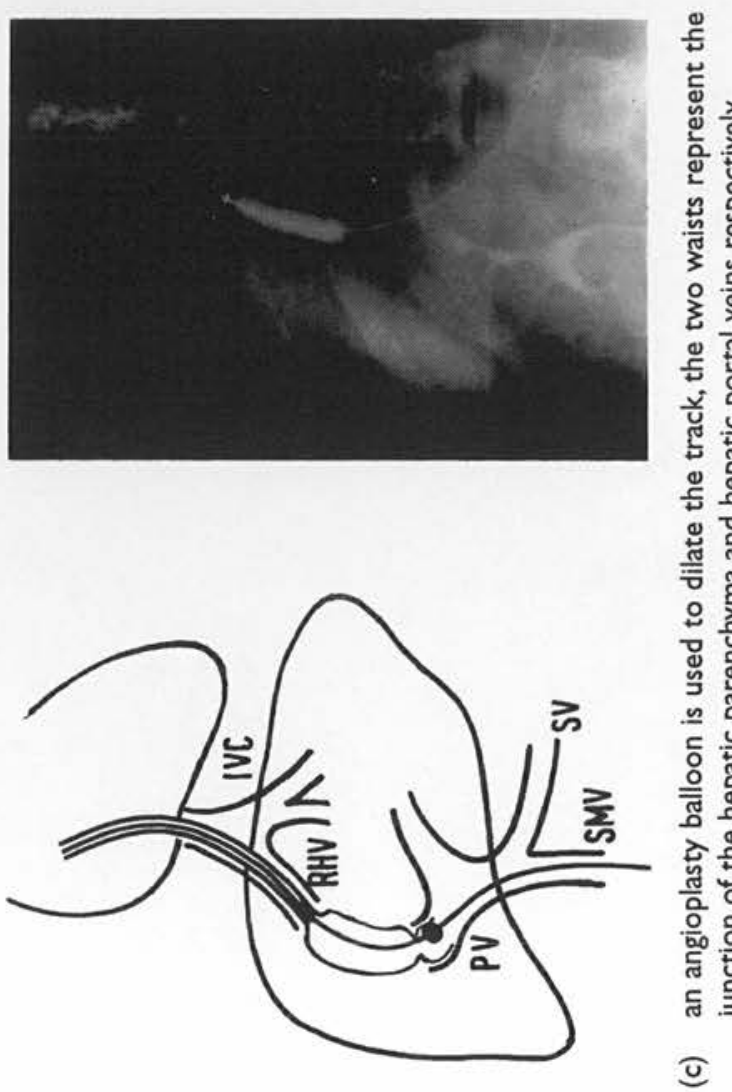
discharge from the hospital and using portography 3 months after TIPSS had been inserted. After the first fifteen patients schedule of follow up was regularised with shunt function being assessed using Doppler the day after TIPSS and using portography at between 6 and 12 weeks and then at 6 and 12 months and then yearly thereafter.

Doppler ultrasound has been used for the assessment of shunt function and a flow in the shunt of less than $1000 \mathrm{mls} . / \mathrm{min}$ and a portal flow velocity of less than $10 \mathrm{~cm} / \mathrm{sec}$ has been suggested as indicating shunt insufficiency (Rossle et.al. 1994). The use of this technique as the principal means of follow up has however, been unsatisfactory in our hands. Portography with direct pressure measurements was considered the gold standard for assessing shunt function. The preparation was exactly the same as the original TIPSS procedure although antibiotics were not routinely prescribed. Following cannulation of the right internal jugular vein the shunt was catherised using the Terumo wire as described above. Portal pressure and inferior vena caval pressures were determined directly. Following this a portography was performed. 


\subsection{Patient details, Follow up and evaluation}

\section{Patients}

The population of patients included into this evaluation were consecutive patients who underwent the TIPSS procedure between mid 1991 and end 1993. Details of the patients included in this study are summarised in Table 2.1. Seventy six cirrhotic patients were referred for TIPSS for variceal haemorrhage. Sixty eight patients $(89.5 \%)$ who had TIPSS inserted successfully were the subjects of this study. Mean age was $54.8 \mathrm{yr}( \pm 10.4)$; 41 were males and 27 females. TIPSS was performed for variceal haemorrhage; from oesophageal varices in 51 and oesophagogastric varices in 17. Forty one patients had associated ascites and 14 had clinically detectable hepatic encephalopathy (Parson-Smith et.al. 1957) prior to TIPSS. Ten patients were ventilated mechanically (6 for hypoxia from aspiration pneumonia and 4 for severe hepatic encephalopathy), 10 had active infection and 15 patients were haemodynamically unstable (pulse $>110 / \mathrm{min}$. or systolic blood pressure $<90 \mathrm{mmHg}$ ) at the time of TIPSS.

Mean haemoglobin was $9.4 \mathrm{~g} / \mathrm{dl}( \pm 1.2)$, prothrombin time was 19.4 $\sec ( \pm 2.4)$ (control $12 \mathrm{sec}$ ), bilirubin was 96.4 umol/l $( \pm 4.3)$ and serum albumin was $29.8 \mathrm{~g} / \mathrm{l}( \pm 3.4)$. Seventeen patients were Child Class A, 30 were Class B and 21 were Class C. Cirrhosis was due to alcohol in 47, primary biliary cirrhosis in 7, cryptogenic cirrhosis in 7 , hepatitis $B$ or $C$ virus infection in 4 , autoimmune chronic active hepatitis in 1, and cystic fibrosis in 2. 
Table 2.1

Patient Characteristics ( $n=68)$

\begin{tabular}{|c|c|}
\hline Age (years) & $56.3( \pm 10.4)$ \\
\hline $\operatorname{Sex}(M / F)$ & $41 / 27$ \\
\hline \multicolumn{2}{|l|}{ Aetiology of Liver Disease } \\
\hline $\begin{array}{l}\text { Alcoholic cirrhosis } \\
\text { Primary Biliary Cirrhosis } \\
\text { Cryptogenic Cirrhosis } \\
\text { Hepatitis B/C } \\
\text { Other }\end{array}$ & $\begin{array}{l}47 \\
07 \\
07 \\
04 \\
03\end{array}$ \\
\hline Pugh Score & $9.7( \pm 2.7)$ \\
\hline \multicolumn{2}{|l|}{ Child class } \\
\hline $\begin{array}{l}\text { A } \\
\text { B } \\
\text { C }\end{array}$ & $\begin{array}{l}17 \\
30 \\
21\end{array}$ \\
\hline \multicolumn{2}{|l|}{ Indication } \\
\hline $\begin{array}{l}\text { Variceal bleeding (uncontrolled) } \\
\text { Variceal bleeding (recurrent) } \\
\text { Refractory ascites } \\
\text { Portal hypertensive gastropathy } \\
\text { Others }\end{array}$ & $\begin{array}{l}18 \\
34 \\
06 \\
06 \\
03\end{array}$ \\
\hline \multicolumn{2}{|l|}{ Clinical Features } \\
\hline $\begin{array}{l}\text { Mechanical ventilation } \\
\text { Haemodynamic stability } \\
\text { Ascites } \\
\text { Hepatic encephalopathy } \\
\text { Sepsis }\end{array}$ & $\begin{array}{l}05 \\
08 \\
41 \\
14 \\
07\end{array}$ \\
\hline \multicolumn{2}{|l|}{ Laboratory data } \\
\hline $\begin{array}{l}\text { Haemoglobin }(\mathrm{g} / \mathrm{l}) \\
\text { Prothrombin time }(\mathrm{s}) \\
\text { Bilirubin }(\mu \mathrm{mol} / \mathrm{l}) \\
\text { Albumin }(\mathrm{g} / \mathrm{l})\end{array}$ & $\begin{array}{c}9.4( \pm 1.2) \\
19.6( \pm) \\
96.4( \pm 5.4) \\
26.8( \pm 3.4)\end{array}$ \\
\hline
\end{tabular}

Results expressed as mean and SD

2 patients were not cirrhotics (amyloidosis and non-cirrhotic portal fibrosis) 


\section{Follow Up And Evaluation}

The patients were discharged 1 week after the procedure if there were no complications and followed up clinically, biochemically and radiologically as described. Patients were followed up for a mean of 10.8 months $( \pm 3.1)$ with a range of 0.1 to 40 months.

In the absence of clinical encephalopathy the patients were allowed a normal diet and lactulose was not used prophylactically. At every visit they had complete neurological examination and encephalopathy was diagnosed according to Parson-Smith et.al. (1957). Psychometric tests were not routinely used. Episodes of encephalopathy were managed with protein restriction and lactulose, reduction in the size of the shunt was required in 2 patients.

\section{Definitions}

Rebleeding Subsequent upper gastrointestinal bleeding manifested by haematemesis and/or malena, with a reduction in haemoglobin by $20 \mathrm{~g} / \mathrm{l}$ and requiring an unscheduled endoscopy. It was classified as originating from oesophageal varices if the patient was bleeding from varices at the time of endoscopy had large oesophageal varices with blood in the stomach and no other recognisable cause of bleeding. Alternatively, the site of rebleeding was noted.

Uncontrolled variceal bleeding Continued bleeding despite 2 separate sessions of banding/sclerotherapy within five days of the first treatment. 
Early mortality Death within 6 weeks of the initial episode of bleeding.

Hepatic encephalopathy Detection of, or deterioration in the grade of encephalopathy (Parson-Smith et.al. 1957).

\subsection{Statistical methods in the assessment of outcome and prognosis.}

Thirty variables related to clinical or biological data or to the importance of haemorrhage were collected on the day of the TIPSS procedure. Sixteen were quantitative: age (yr), Child Class, haemoglobin $(\mathrm{g} / \mathrm{dl})$, leucocyte count (II), platelet count (II) prothrombin time (sec, control 12), serum alanine aminotransaminase (IU/l), bilirubin (umol/l), alkaline phosphatase (IU/l), albumin $(\mathrm{g} / \mathrm{l})$, sodium $(\mathrm{mmol} / \mathrm{l})$, urea $(\mathrm{mmol} / \mathrm{l})$ and creatinine (umol$/ \mathrm{l})$, size of the shunt used $(\mathrm{mm})$ and the initial and final portal pressure gradients $(\mathrm{mmHg})$. These were expressed as means and standard deviations. The qualitative variables studied were, sex, aetiology of underlying liver disease, indication for TIPSS, active alcoholism, emergency or elective TIPSS, need for mechanical ventilation, haemodynamic stability (systolic blood pressure $<90 \mathrm{mmHg}$, or resting pulse rate $>110$ beats/min), active infection, ascites (none, mild-moderate or severe), clinically detectable encephalopathy (Parson-Smith criteria), nutritional status, presence of associated diseases and the type of stent used.

The univariate association between individual prognostic factors and post treatment encephalopathy was tested by Chi squared or Wilcoxon rank 
sum tests as appropriate and multiple logistic regression was used to test the significance of factors adjusted for one another. Cox's proportional hazard regression was used to test the univariate and multivariate significance of prognostic factors in relation to the other 3 outcomes. All 3 survival outcomes were censored when the patients underwent liver transplantation; shunt failure and rebleeding were also censored at death. 


\subsection{Mental state and neuropsychological tests}

\section{Study population}

The population studied comprised of 3 groups.

Group I Patients undergoing TIPSS for recurrent variceal haemorrhage that was difficult to control with variceal band ligation.

Group II Patients presenting with variceal haemorrhage and enrolled into a programme of band ligation and

Group III Age and sex matched healthy volunteers acted as controls.

Characteristics of patients in the TIPSS and band ligation groups are summarised in Table 2.2 .

\section{Patients treated with TIPSS}

Twenty nine patients undergoing TIPSS for prevention of recurrent variceal haemorrhage were included in this study. The mean number of sclerotherapy sessions they had was $2.1( \pm 1.2)$. Patient characteristics are summarised on Table 2.2. Patients were only included in the study if they showed no evidence of clinically detectable encephalopathy (Parson-Smith et.al. 1957). All patients at the time of assessment were haemodynamically stable, had not bled during the week leading up to TIPSS, were on no neurotropic or vasoactive medications, had no renal failure and had not had any sedation of any kind within 3 days of the TIPSS. They were enrolled between 4 and 26 days after the last variceal bleed (mean $8.6( \pm 2.7)$ days). Prophylactic lactulose or protein restriction was not used. Fifteen patients were recruited into the study prior to TIPSS, 14 others were assessed at 1 month, 26 of 
these patients were assessed at 3 months, 24 at 9 months and 23 at 15 months

\section{CONTROLS}

Normal healthy volunteers

Sixteen healthy volunteers with a mean age of $46( \pm 3.1)$ on no medications acted as controls.

\section{Cirrhotic controls}

Twelve patients with cirrhosis and variceal haemorrhage being treated with variceal band ligation for prevention of recurrent variceal haemorrhage were recruited as controls. Mean number of sclerotherapy sessions they had prior to entry into the study was $1.6( \pm 2.2)$. They were enrolled into a programme of variceal band ligation following the index haemorrhage. Mean time from their last bleed to the time of entry into the study was 5.1 days $( \pm 2.7)$. These patients had no clinically detectable encephalopathy (Parson-Smith et.al. 1957), were on no vasoactive or neurotropic medications, were haemodynamically stable and had no evidence of renal failure. 


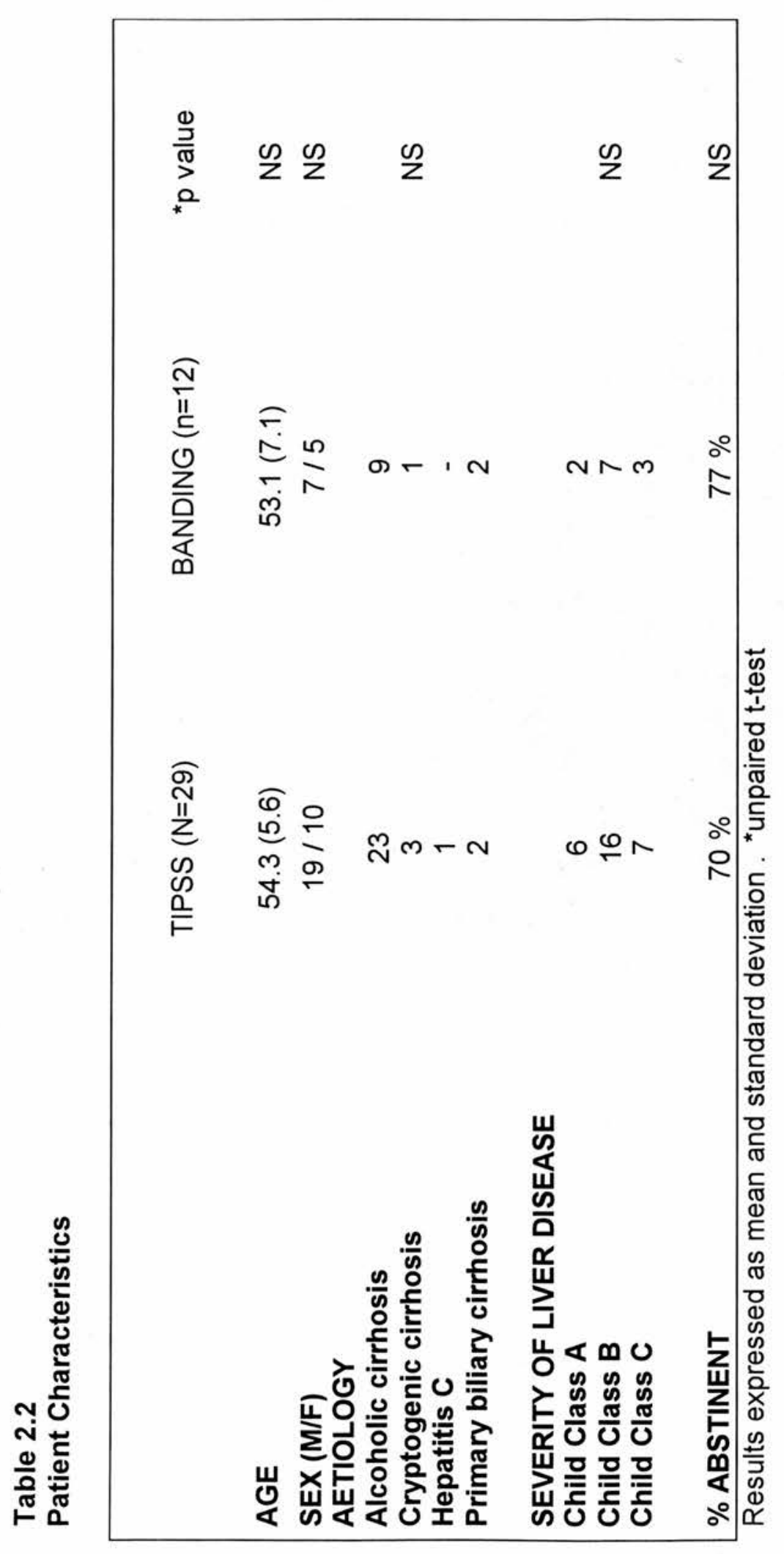




\section{Methods Of Treatment}

TIPSS

TIPSS was performed according to the method outlined in section 3.21. Shunt function was assessed at 1, 3 and 9 months and yearly thereafter.

\section{Variceal band ligation}

Variceal band ligation was performed using the Bard ligator at 1-2 week intervals until variceal eradication and then at 3 months and 6 monthly thereafter.

\section{Assessment Of Neuropsychological Function}

The following battery of tests was used in the assessment of neuropsychological function and quality of life. They were performed at the time of enrollment of the patients into the study and then at 1,3 9 and 15 months.

Cambridge Automated Neuropsychological Assessment Battery (CANTAB) This is a sensitive instrument for carrying out detailed neuropsychological assessment. It incorporates measures, amongst others, of delayed matching to sample memory test, and simple and complex reaction times. A touch sensitive VDU screen is used for accurate recording of responses and reaction times. It has been shown to be a valid, reliable and sensitive measure of neuropsychological function (Owen et.al. 1991). It has the advantage of having 4 parallel forms which allows repetitive measurements 
and has been shown to be useful in a large study assessing the efficacy of cognitive enhancers in Alzheimer's disease.

Rivermead Behavioral Memory Test (RBMT)

This 'ecologically valid' measure assesses the memory problems encountered by patients in their everyday lives and consists of a number of sub-tests each of which provides a measure of everyday problems such as remembering an appointment, remembering a short route, remembering to deliver a message, etc. This also has 4 parallel forms which serves to eliminate the effects of learning (Wilson et.al. 1989).

Hospital Anxiety and Depression Scale (HAD)

This is a well validated measure of anxiety and depression which was designed for use in medically ill patients. Despite the use of the word 'hospital' in the title, it is valid even for community work. It assesses anxiety and depression separately and consists of 7 depression items and 7 anxiety items (Zigmond et. al. 1983). Care has been taken to eliminate symptoms which can be present in both anxiety and in depression. The validity of this test has been corroborated by other workers (Moorey et.al. 1991).

Quality of Life (QOL)

This was measured using a modified version of Rotterdam Symptom Checklist (Trew M and Maguire $P, 1984$ ) which assesses physical and psychological symptoms separately. It was developed to assess symptoms in 
cancer patients and was constructed on the basis of analysis of data from 3 different studies done with 3 different checklists (Pruyn JFA 1980, Linssen et.al 1979, McCorkle R and Young $\mathrm{K}$ 1978). Its validity and stability in the assessment of both psychological and physical dimensions has been confirmed in an independent study (de Haes et.al. 1990).

\section{Assessment Of Liver Function}

Routine tests of liver function and hepatocellular injury

Changes in serum albumin, prothrombin time, alanine transaminase (ALT) and bilirubin were measured at enrollment, after 7 days, 1 and 3 months and 6 monthly thereafter in patients in both the banding and the TIPSS groups.

Measurement of Indocyanine green (ICG) clearance ICG clearance was measured using the method described previously (Jalan et.al. 1994) prior to TIPSS, 1 week and 3 months after TIPSS in the 15 patients who were recruited into the study prior to TIPSS. Briefly, a bolus injection of $0.5 \mathrm{mg}$ per $\mathrm{kg}$ was used for measuring clearance. Venous blood samples were collected from an indwelling cannula inserted into a peripheral vein at 5 minute intervals for 20 minutes, concentration was measured spectrophotometrically and transformed logarithmically. Volume of distribution (VD) was calculated as the ratio of the dose to the concentration at time 0 (extrapolated from the graph). The elimination rate constant $(K)$ was derived from the half life time and the slope of the curve. Clearance was 
measured as the product of the volume of distribution and the elimination rate $($ clearance $=\mathrm{VD} \times \mathrm{K})$.

\section{Follow Up And Analysis}

All the patients enrolled into the study were assessed routinely by a designated team. The patients have been followed up with regular portography to assess shunt patency, assessment of neuropsychological function and quality of life and measure of liver function tests. Results were expressed as mean and standard error. Differences within groups were calculated using Wilcoxon signed-ranks comparing values at baseline with the values at subsequent time points and between groups using unpaired ttests. Correlation between changes in different parameters was calculated using linear regression. Subclinical encephalopathy was operationally defined in a patient if the value for the subtests of CANTAB (delayed matching to sample memory test, and simple or complex reaction times) and/or RBMT fell outside 2 standard deviations of the control mean. 


\subsection{Haematological assessment}

\section{Patients}

Twenty three patients with TIPSS with a minimum duration of follow up of 3 months were included in this study; 13 of the 23 were males, mean age was 53.6 (se 1.7). Eight were Child grade A, 7 were grade $B$ and 8 were grade $C$. Eighteen patients had alcoholic cirrhosis, cryptogenic cirrhosis (3), Hepatitis B (1) and Hepatitis C (1). Indications for TIPSS were prevention of recurrent variceal haemorrhage in 18 , refractory ascites in 2 , hypersplenism in 1 and portal hypertensive gastropathy in 2 . All the patients were stable without any evidence of continued haemorrhage at the time of the procedure, had no clinically apparent infection or renal failure and were on no drugs known to cause haemolysis. No patients required transfusion of blood or blood products for at least 48 hours prior to or for seven days following TIPSS.

\section{TIPSS}

TIPSS was performed according to the method outlined in section 3.21. Shunt function was assessed at 1, 3 and 6 months and yearly thereafter.

\section{Haematological Assessment}

Complete haematological assessment was made prior to, at 3,7 and 30 days following TIPSS and 3 monthly thereafter.

Full blood count Red and white cell counts, platelet count, mean cell volume, mean corpuscular haemoglobin and erythrocyte count were measured using 
routine methods. Red cell morphology was assessed to look for evidence of haemolysis.

Assessment of haemolysis Reticulocyte count (measured automatically), serum haptoglobin (measured electrophoretically), urinary haemosiderin (early morning sample), red cell morphology and Coombs test were used to assess haemolysis.

Spleen size was measured using ultrasonography and the maximum length was taken as the representative value.

\section{Follow Up And Analysis}

The patients were routinely followed up clinically at 1,3 and 6 months and 3 monthly thereafter. Complete clinical, haematological and biochemical examination was performed at this stage. Doppler ultrasonography and routine portogram with the measurement of portal pressure gradient was performed at 3 and 6 months and 6 monthly thereafter. Results were expressed as means and standard error. Differences between groups were tested using ANOVA and correlations sought using linear regression. A p value of less than 0.05 was considered as being significant. 


\subsection{RESULTS}

\subsection{TIPSS insertion}

These are summarised in Table 2.3. The TIPSS procedure was unsuccessful in 8 patients because of failure to puncture the portal vein branch satisfactorily. At the time of TIPSS 8 patients were still haemodynamically unstable (Palmaz stent (PS)-3, Wallstent (WS) $-5, p=N S$ ) and 5 required mechanical ventilation (PS-2, WS-3, P=NS). Twelve of the 16 patients in whom the shunt was successfully inserted as an emergency procedure had WS $(P<0.01)$.

The mean portal pressure gradient before TIPSS was similar in both groups (PS-22 and WS-19, $\mathrm{p}=\mathrm{NS}$ ). The mean size of the stents required to produce adequate reduction in the portal pressure gradients in the 2 groups were similar. With similar sized shunts the reduction in the portal pressure gradient in the WS group was significantly greater than in the PS group $(p<0.01)$. The mean number of stents required for shunt coverage was significantly lower in the WS group $(p<0.001)$ (Table 2.3).

Two patients died of procedure related complications from intraperitoneal bleeding. One in the PS group was related to bleeding from extrahepatic puncture of the portal vein bifurcation. The other in the WS group was related to bleeding from the liver after breach of the liver capsule.

Clinically significant complications either in the form of variceal rebleeding or reaccumulation of ascites were significantly higher in the PS group $(p<0.01)$ (Figure 2.2). Similarly, asymptomatic complications were also 
significantly greater $(p<0.01)$. Frequent problems were encountered with the PS at the time of shunt deployment or shortly thereafter with significantly higher rates of portal vein thrombosis, dislocated stent (Fig 2.3) and stent migration (Fig 2.4). The overall incidence of hepatic vein stenosis (Fig 2.5) was similar in both groups but the rate of shunt stenosis due to intimal hyperplasia (Fig 2.6) was significantly higher in the PS group $(p<0.001)$.

Problems in the management of stent complications occurred primarily in the PS group. This was manifested by frequent rupture of the angioplasty balloons by the frayed ends of the wire mesh. On 2 occasions the angioplasty balloon ruptured and was caught on the wire mesh. This resulted in major problems; in 1 case insertion of a large caliber sheath over the balloon allowed withdrawal, in the other manipulation of the balloon caused it to break. Most but not all of the catheter was recovered with the use of a goose neck snare and WS inserted through the shunt to trap the remaining fragment (Fig 2.7). 
Kaplan Meier Analysis Of Variceal Rebleeding Following TIPSS

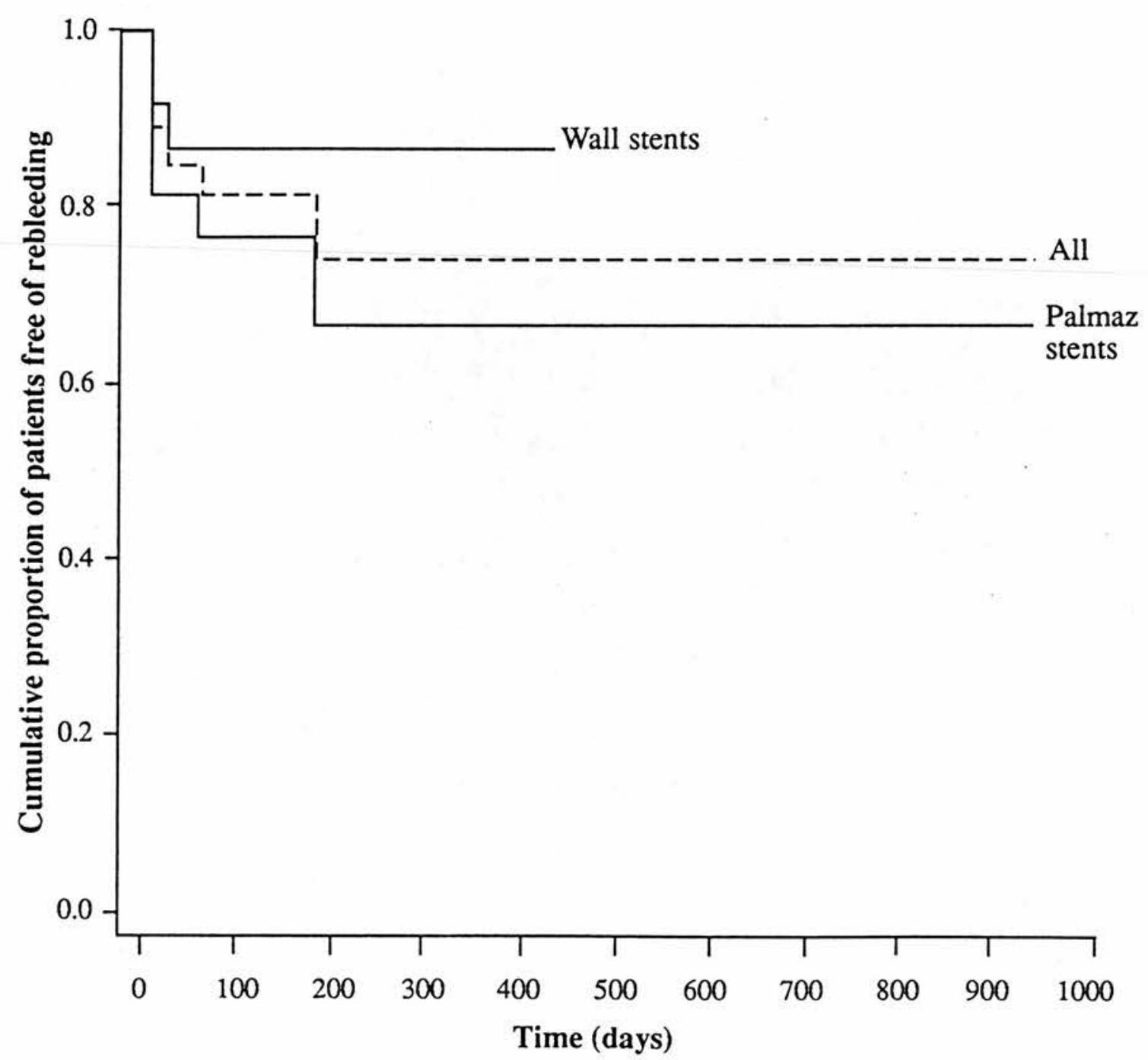

Figure 2.2 


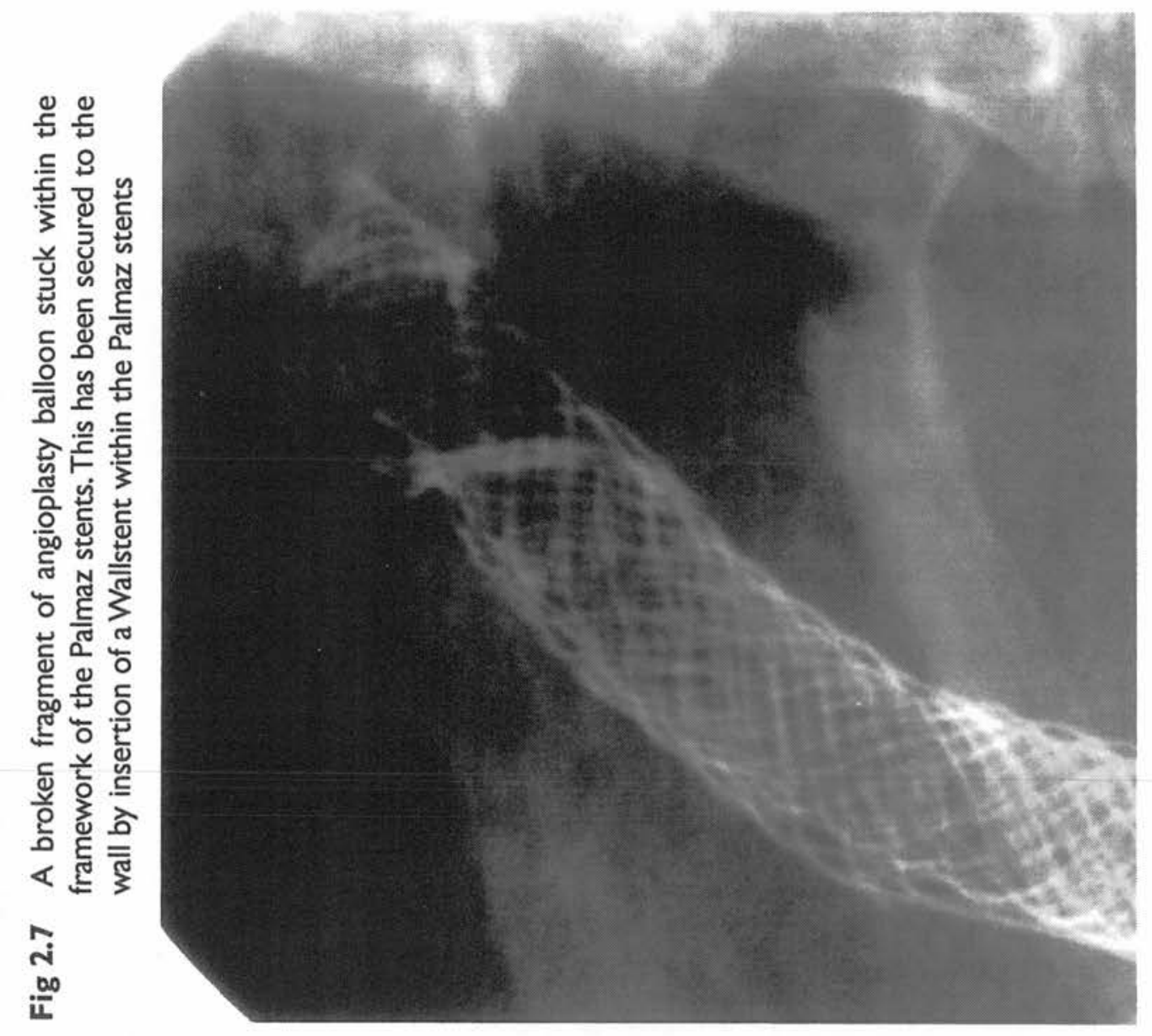

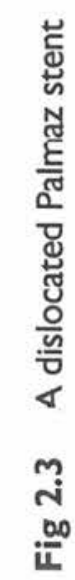

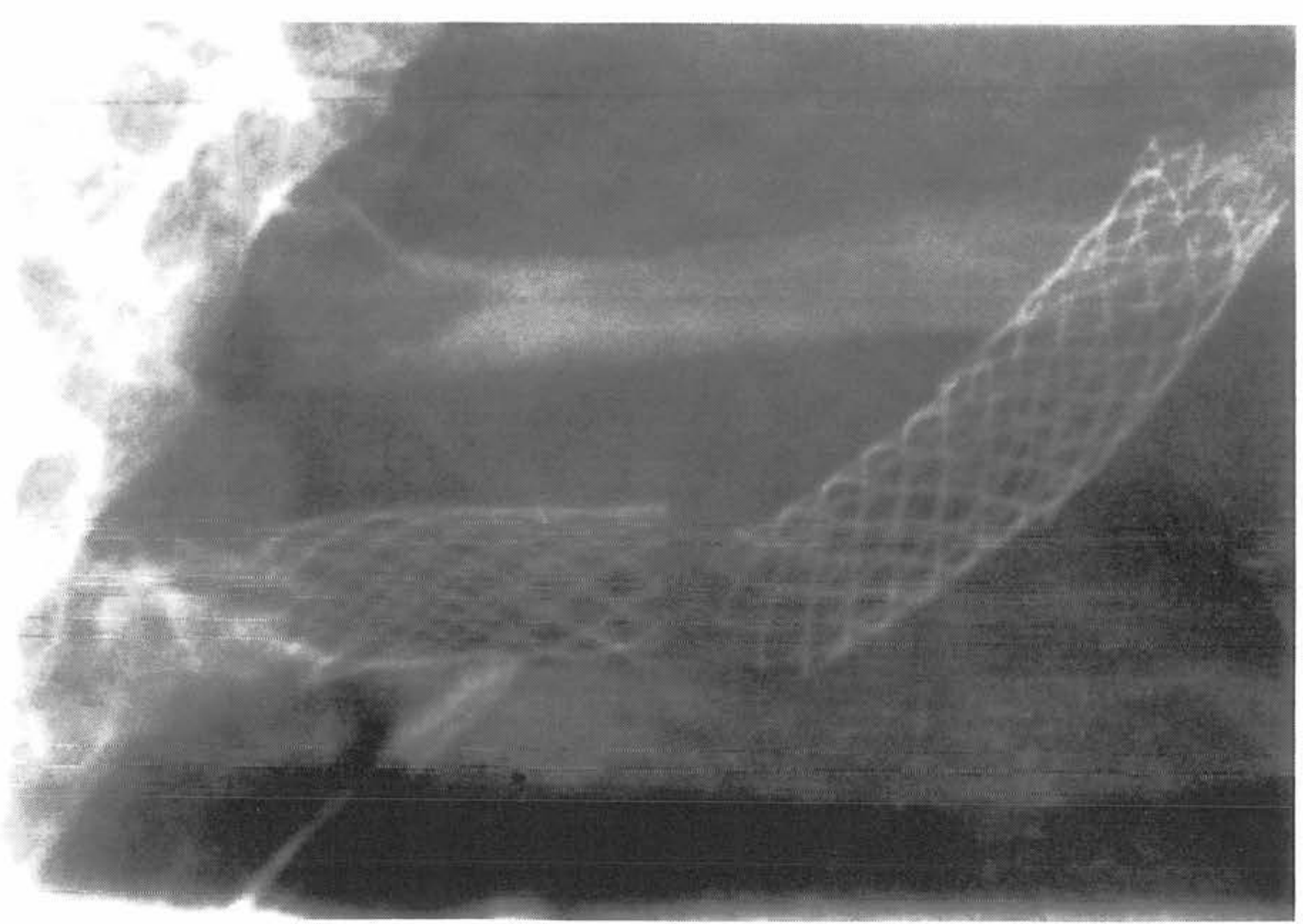


Fig 2.4 A Palmaz stent that has migrated from the parenchymal track into the trunk of the portal vein.

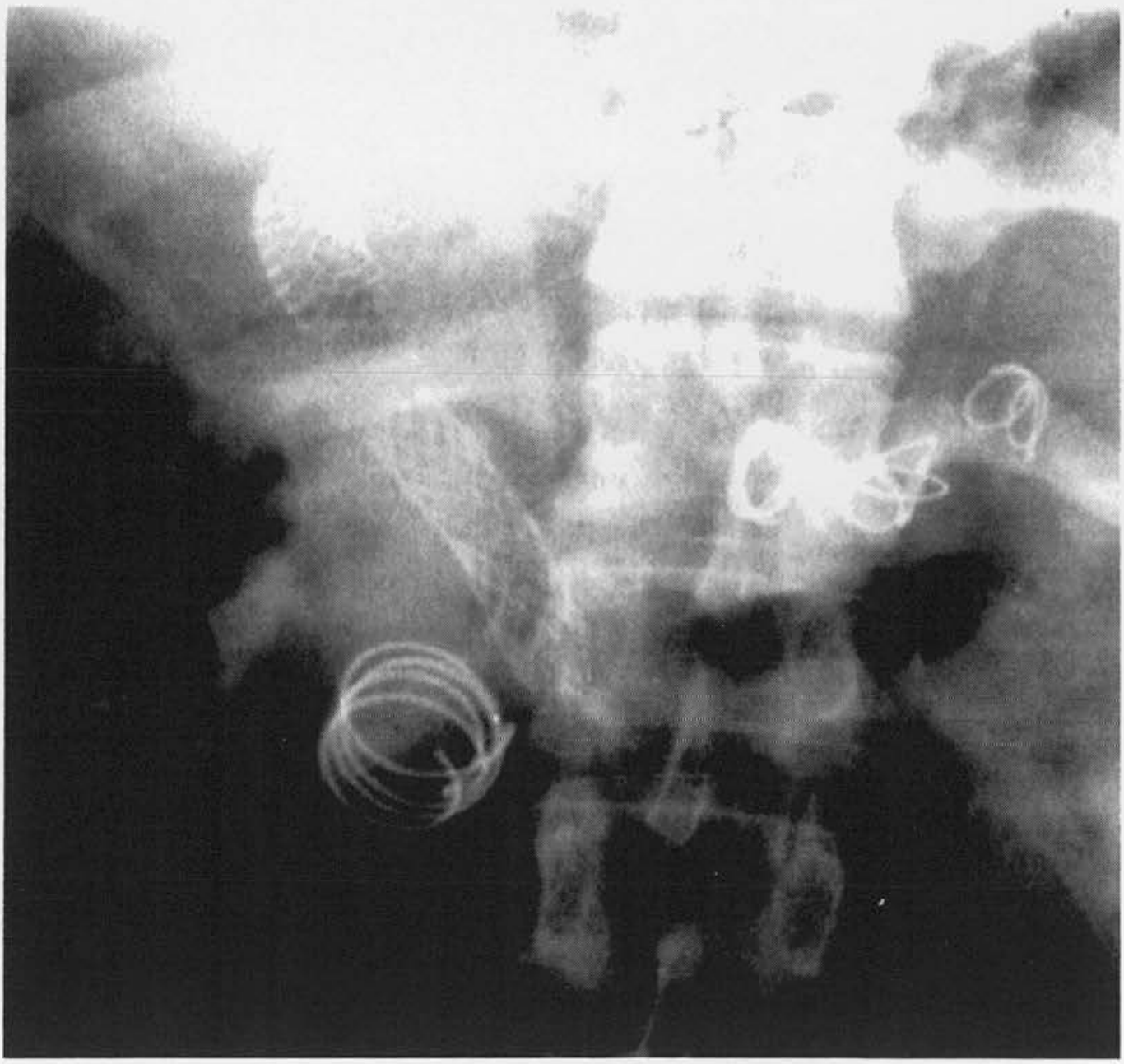


Fig 2.5 Hepatic vein stenosis producing recurrence of varices.

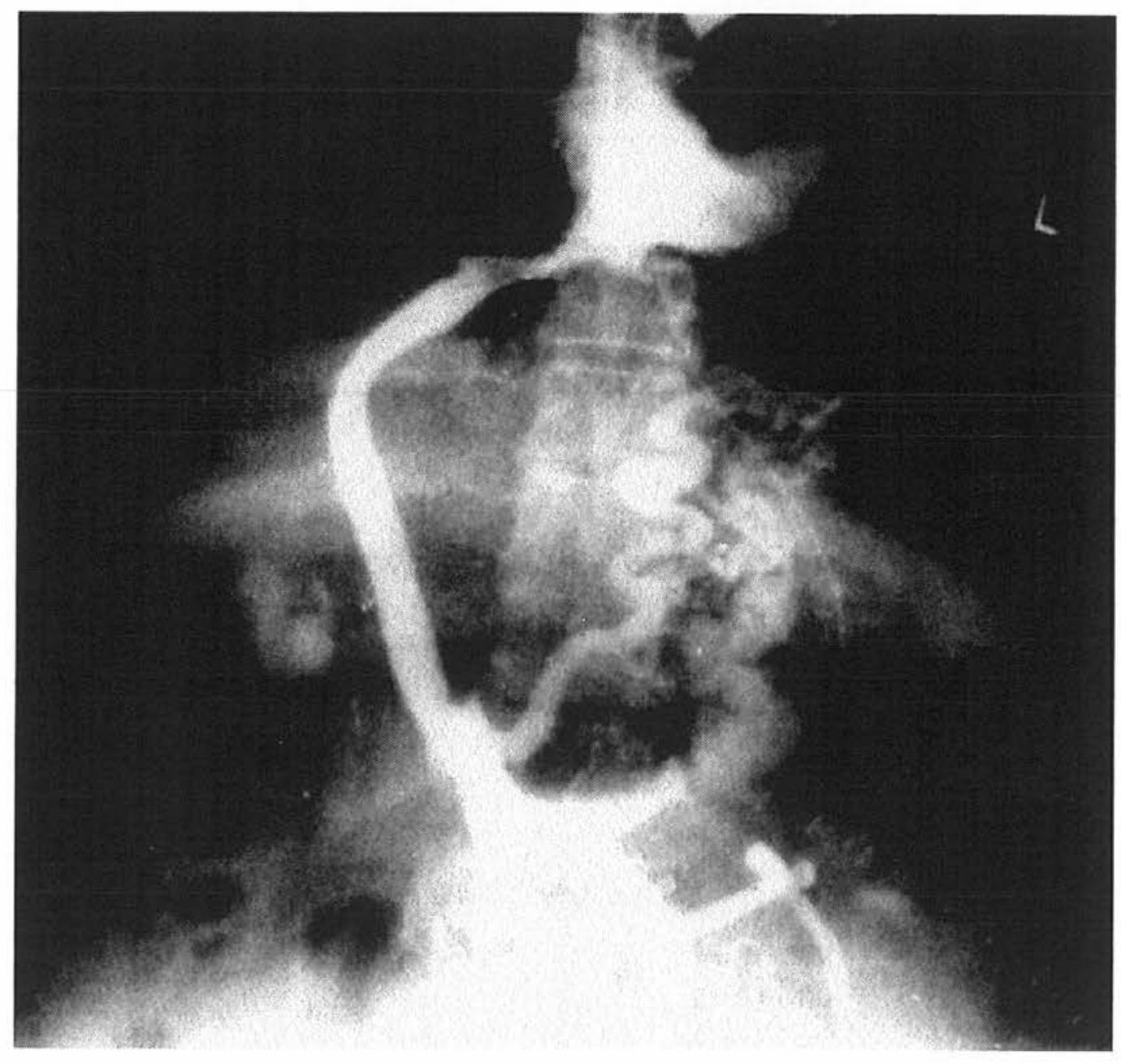


Fig 2.6 Intimal hyperplasia within a Wallstent.

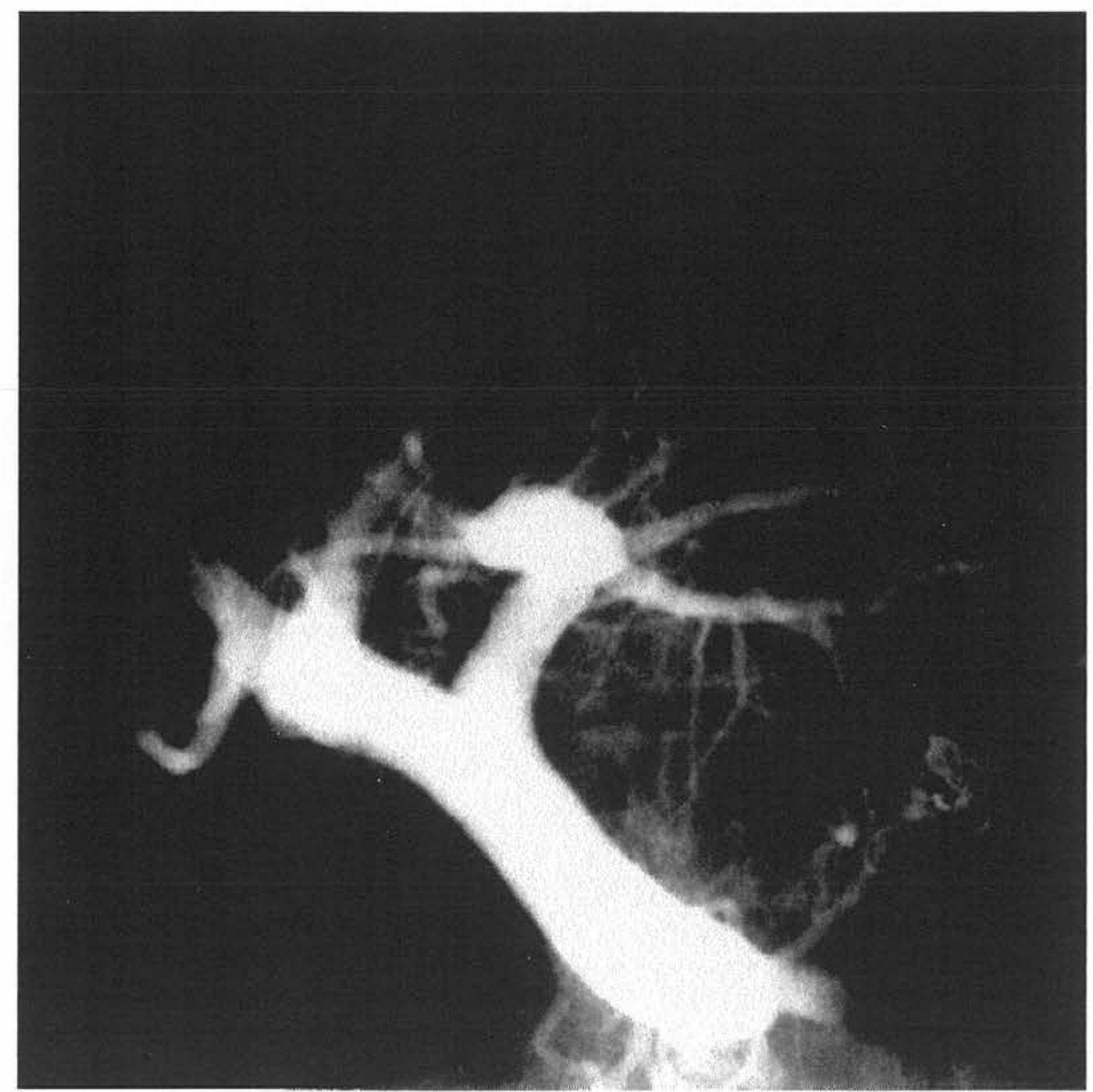


Kaplan Meier Analysis For Survival

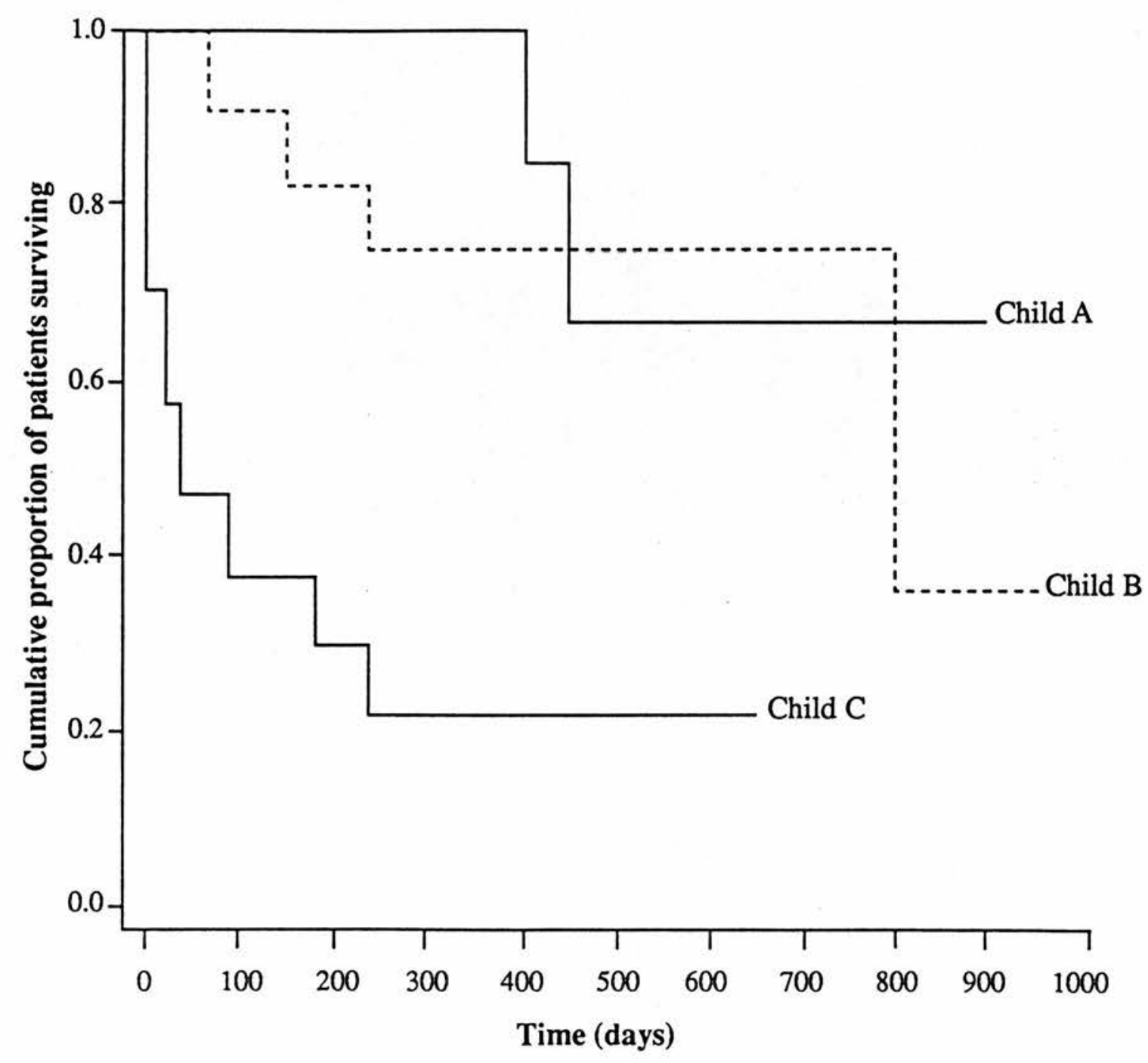

$\begin{array}{ll}\text { Figure } \quad 2.8 & \end{array}$ 


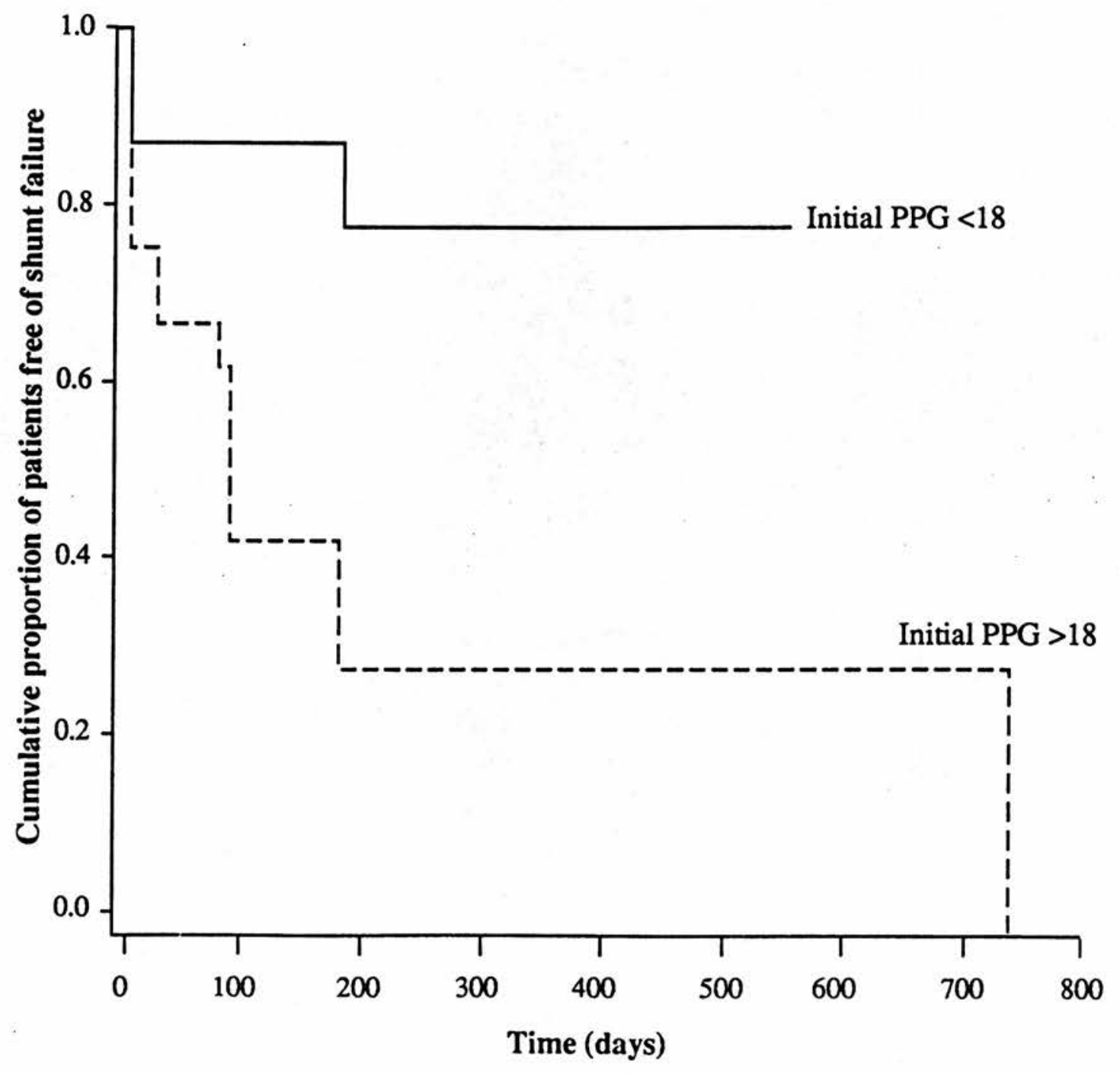

Figure 2.9 
Kaplan Meier Analysis For Shunt Failure

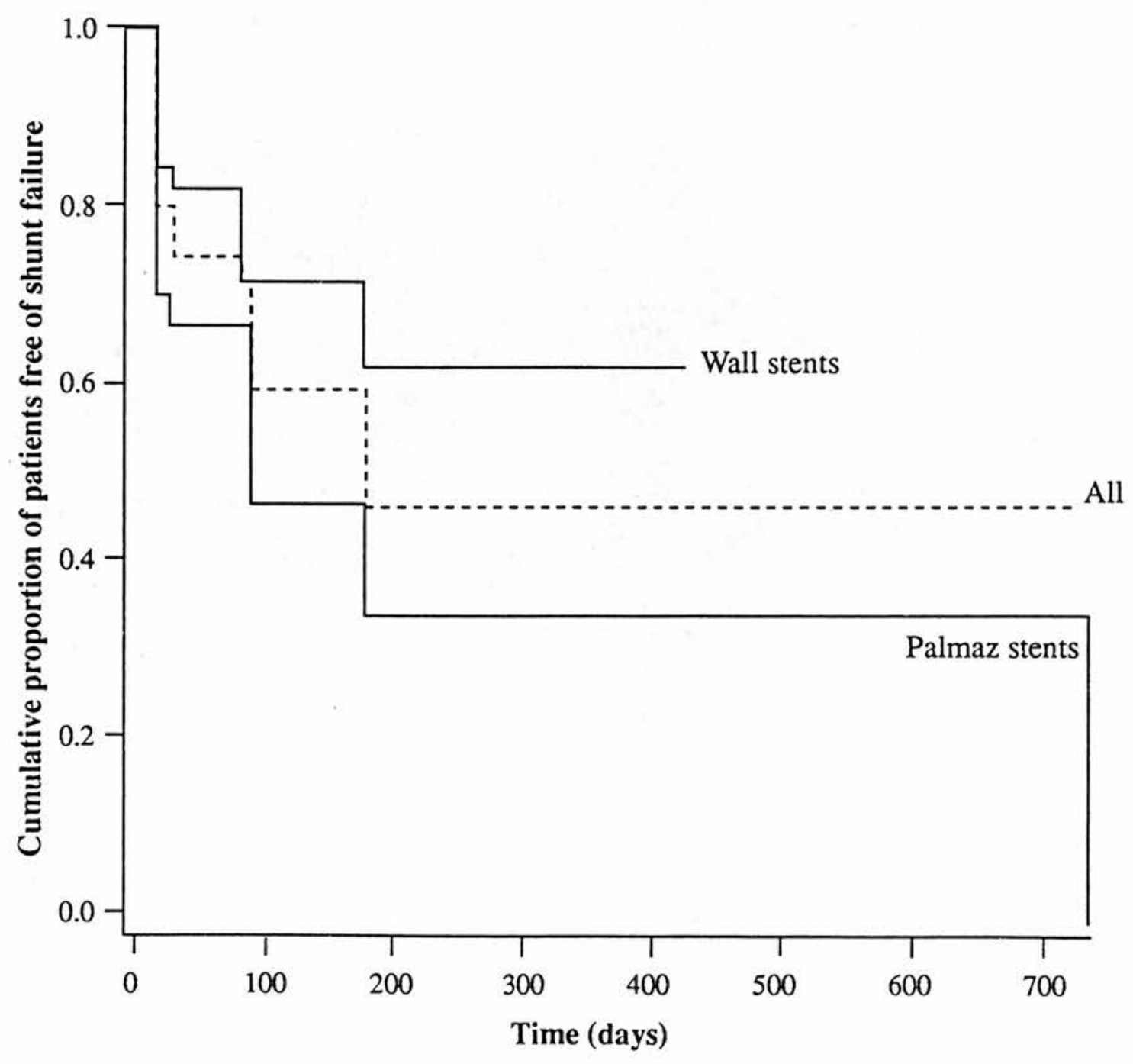

Figure 2.10 
Table 2.3

Results of TIPSS procedures in patients with Palmaz and Wallstents

\begin{tabular}{|c|c|c|c|}
\hline PATIENTS $(n=64)$ & $\begin{array}{l}\text { PALMAZ } \\
\text { STENT }\end{array}$ & $\begin{array}{l}\text { WALL } \\
\text { STENT }\end{array}$ & $p^{*}$ value \\
\hline Successful TIPSS & $24 / 28(85.7 \%)$ & $32 / 36(88 \%)$ & NS \\
\hline Initial PPG (mmHg) & $22( \pm 6)$ & $19( \pm 5.4)$ & NS \\
\hline Final PPG $(\mathrm{mmHg})$ & $12.4( \pm 2.9)$ & $8.6( \pm 3.6)$ & $<0.01$ \\
\hline Size of stents (mm) & $12( \pm 1.5)$ & $12.5( \pm 1.1)$ & NS \\
\hline Number of stents & $2.8( \pm 0.6)$ & $1.3( \pm 0.4)$ & $<0.001$ \\
\hline Procedure related death & 1 & 1 & NS \\
\hline $\begin{array}{l}\text { Clinically significant } \\
\text { complications }\end{array}$ & $10(41.6 \%)$ & $5(15.6 \%)$ & $<0.01$ \\
\hline $\begin{array}{l}\text { Diagnosed on surveillance } \\
\text { (shunt events) }\end{array}$ & $16(66.6 \%)$ & $5(15.6 \%)$ & $<0.01$ \\
\hline Overall (patients) & $20(83.2 \%)$ & $10(31.2 \%)$ & $<0.01$ \\
\hline Type of complication: & & & \\
\hline $\begin{array}{l}\text { Portal vein/ shunt } \\
\text { thrombosis } \\
\text { Intimal Hyperplasia } \\
\text { Hepatic vein stenosis } \\
\text { Other }\end{array}$ & $\begin{array}{c}3 \\
13 \\
5 \\
5\end{array}$ & $\begin{array}{l}1 \\
3 \\
5 \\
1\end{array}$ & $\begin{array}{c}\text { NS } \\
<0.001 \\
N S \\
<0.01\end{array}$ \\
\hline
\end{tabular}

* - two sample t-test, results expressed as mean and SD PPG - Portal pressure gradient 
2.32. Results of patient follow up and evaluation and 2.33 Results of analysis of prognostic variables in the prediction of variceal rebleeding, mortality, encephalopathy and shunt insufficiency following TIPSS

\section{Mortality}

During the period of follow up 22 patients with TIPSS died (14 within 30 days) and 8 underwent orthotopic liver transplantation. Early mortality was related to the procedure in 1 (intraperitoneal bleeding from injury to the portal vein at its bifurcation), acute-on-chronic liver failure in 3 , hepatorenal failure in 2 , end-stage liver disease in 6 and sepsis in 2 . Deaths during follow-up were related to end stage liver disease in 5 , variceal haemorrhage in 1 , bronchopneumonia in 1 and cerebrovascular accident in 1. Kaplan Meier analysis of mortality is depicted in Figure 2.8 .

Prognostic variables predicting early death in the univariate analysis were the severity of liver disease (Child Class), hyponatremia, presence of encephalopathy, bilirubin concentration and serum alanine aminotransaminase (Table 2.4). Only 2 variables retained independent predictive value at multivariate analysis and these were the Child Class and hyponatremia. The risk of early mortality in a Child C cirrhotic who is hyponatremic is about $80 \%$.

Mortality in the long term was predicted in the univariate analysis by Child Class and hyponatremia. Hyponatremia and the presence of encephalopathy prior to TIPSS retained independent predictive value for death in the long term. 


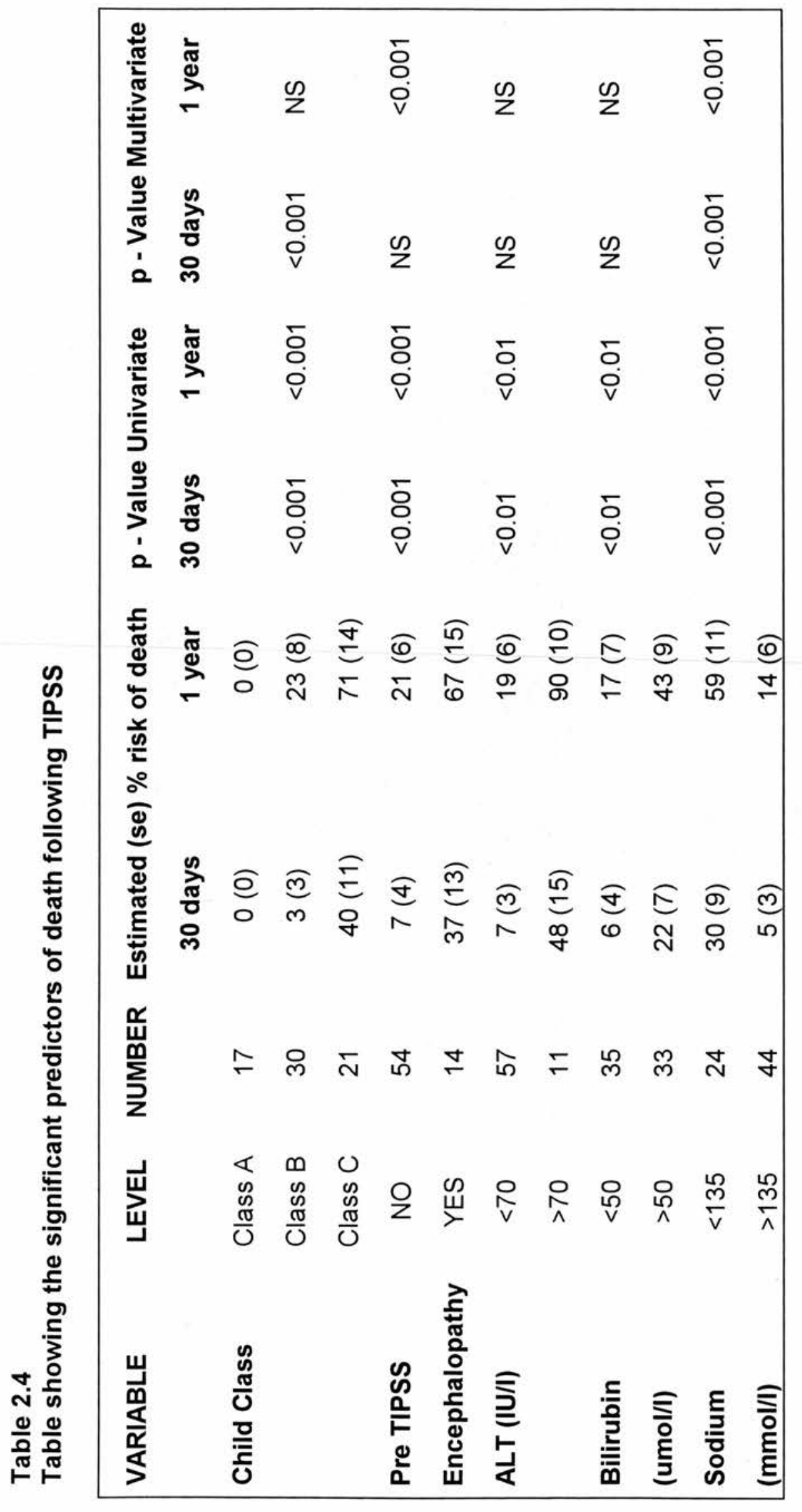




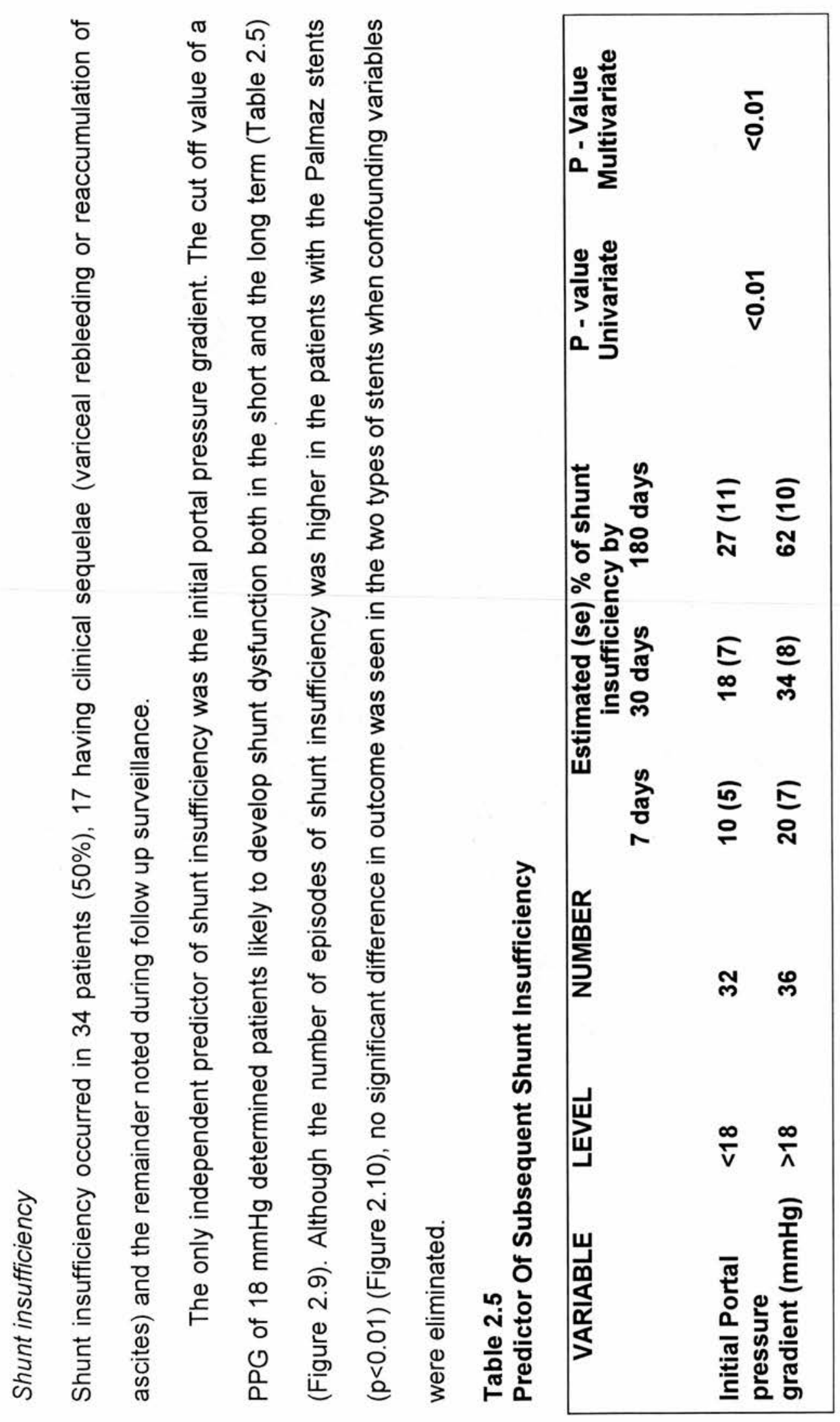




\section{Variceal rebleeding}

Rebleeding was of variceal origin in 14 of the 19 patients who rebled. The 5 episodes of non variceal haemorrhage were from post sclerotherapy oesophageal ulcers in 3 and duodenal ulcers in 2. Variceal rebleeding occurred within the first week of TIPSS in 5 patients, in 9 within 30 days, in 12 within 3 months and in all 14 within 1 year. No variable significantly predicted subsequent variceal haemorrhage. Rebleeding rate was higher in patients with the Palmaz compared with the patients with the Wallstents, although the difference failed to reach statistical significance $(p=0.052)$. All the episodes of variceal haemorrhage were however, associated with portographic and manometric (PPG $>12 \mathrm{mmHg}$ ) evidence of shunt insufficiency.

\section{Encephalopathy}

Twenty one patients (30.9\%) were encephalopathic following TIPSS. In 7 patients $(12.8 \%)$ this was shunt induced (these patients did not have any evidence of hepatic encephalopathy before TIPSS insertion). Statistical significance for encephalopathy following TIPSS on univariate analyses was found with Child Class, the presence of encephalopathy prior to TIPSS, serum albumin, active infection and serum creatinine. When adjustment was made for the pretreatment status, only the presence of encephalopathy prior to TIPSS remained significant (Table 2.6). 


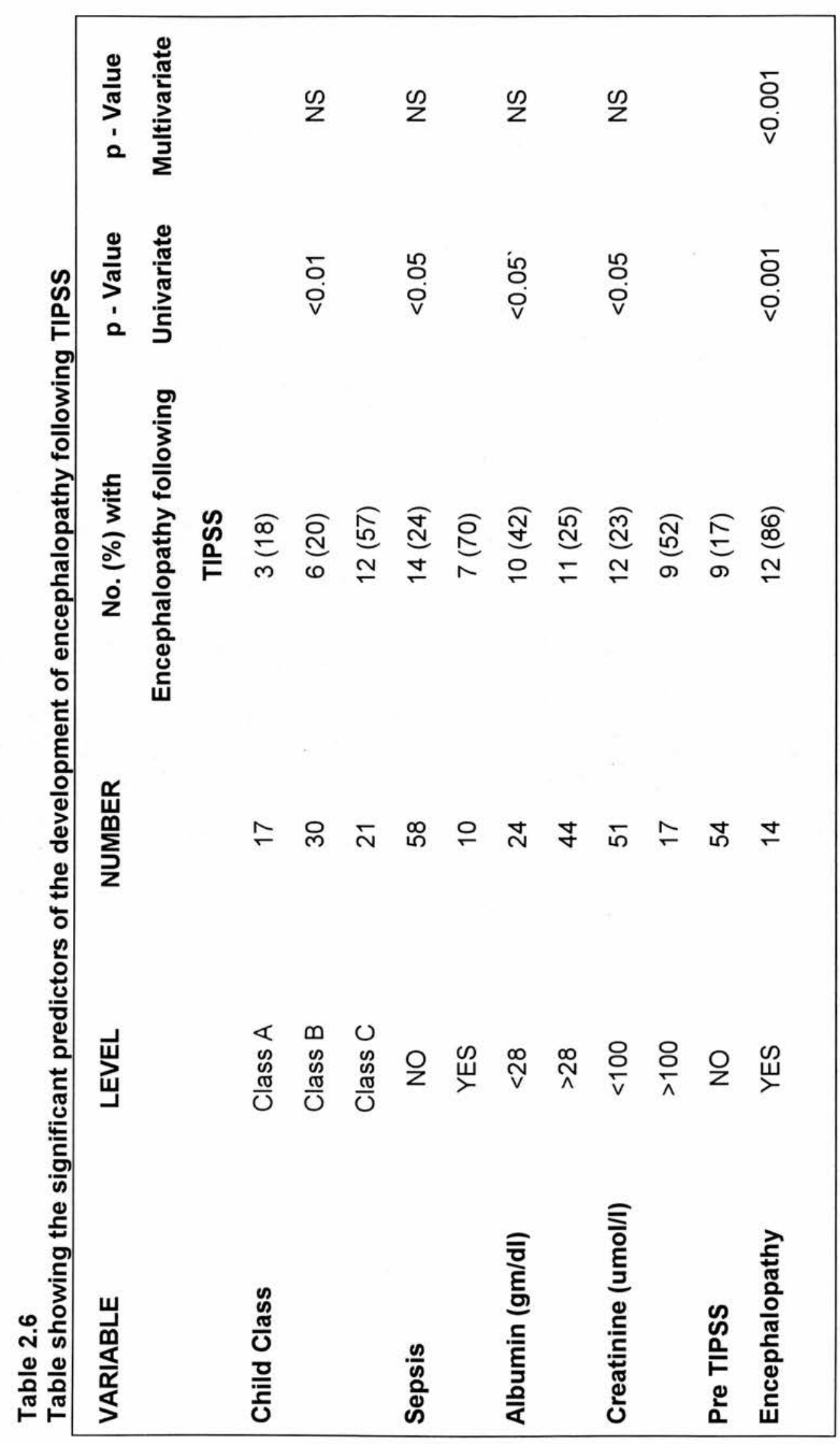




\subsection{Relationship between PPG and variceal haemorrhage}

It is an accepted notion that a portal pressure gradient (PPG) of $>12 \mathrm{mmHg}$ is necessary for the development of and bleeding from oesophagogastric varices although no linear correlation exists between the severity of portal hypertension and the risk of variceal haemorrhage (Garcia-Tsao et.al. 1985, Lebrec et.al. 1980). In a prospective study comparing propranolol with placebo for prevention of first variceal haemorrhage Groszmann et.al. (1990) showed that bleeding from varices did not occur if the hepatic venous pressure gradient (HVPG) could be reduced to less than $12 \mathrm{mmHg}$. He concluded that reducing the portal pressure gradient to $<12 \mathrm{mmHg}$ protects from variceal bleeding, prolongs survival and should be the aim of pharmacological therapy of portal hypertension.

The introduction of transjugular intrahepatic portosystemic stent shunts (TIPSS) in the treatment of variceal haemorrhage allows direct measurement of the portal pressure while performing this technique (Jalan et.al. 1994). The aim of this study was to assess the frequency of variceal haemorrhage in patients with a baseline PPG of $<12 \mathrm{mmHg}$.

Between June 1991 and June 1994, 48 patients who underwent TIPSS for recurrent variceal haemorrhage which was poorly controlled by injection sclerotherapy and/or band ligation were studied. Mean number of sessions of sclerotherapy / band ligation was 3.2 (range 2-5) and the mean duration from the last bleed to the time of TIPSS was 3.9 (range 2-9) days.

The method of insertion of TIPSS has been described in detail elsewhere (Section 3.21). The portal vein was punctured using a thin needle 
(5 Fr.) passed through a transjugular needle and the pressure was measured from the main portal vein following placement of a $5 \mathrm{Fr}$. pigtail catheter into the portal vein. Right atrial, inferior vena caval and portal pressure were recorded and the gradient calculated (portal pressure - inferior vena caval pressure).

PPG was reduced from a mean of $21.4( \pm 6.4)$ prior to TIPSS to 10.6 $( \pm 3.1) \mathrm{mm} \mathrm{Hg}$ following the procedure. Seven patients had a baseline portal pressure gradient of $<12 \mathrm{~mm} \mathrm{Hg}$ (Table 2.7). At the time of the TIPSS all 7 patients were haemodynamically stable (systolic blood pressure $>100 \mathrm{mmHg}$ and heart rate $<100 / \mathrm{min}$ ) and had normal right atrial pressures $(>4 \mathrm{mmHg}$ ). None of them was receiving any vasoactive medications.

This study clearly demonstrates that in $14.7 \%$ patients variceal haemorrhage from oesophagogastric varices occurred despite a PPG of $<12$ $\mathrm{mmHg}$. It is unlikely that this was due to hypovolemia because of the haemodynamic stability and normal central filling pressures.

In the earlier studies the HVPG was used to define the threshold for variceal haemorrhage. We used direct pressure measurements in this study which has been shown to closely reflect the HVPG in alcoholic cirrhosis (Valla et.al. 1984, Boyer et.al. 1977). In patients with cirrhosis and portal hypertension with a significant pre-sinusoidal component the directly measured PPG tends to be higher than the HVPG (Boyer et.al. 1977) and would therefore not explain the present observation. 


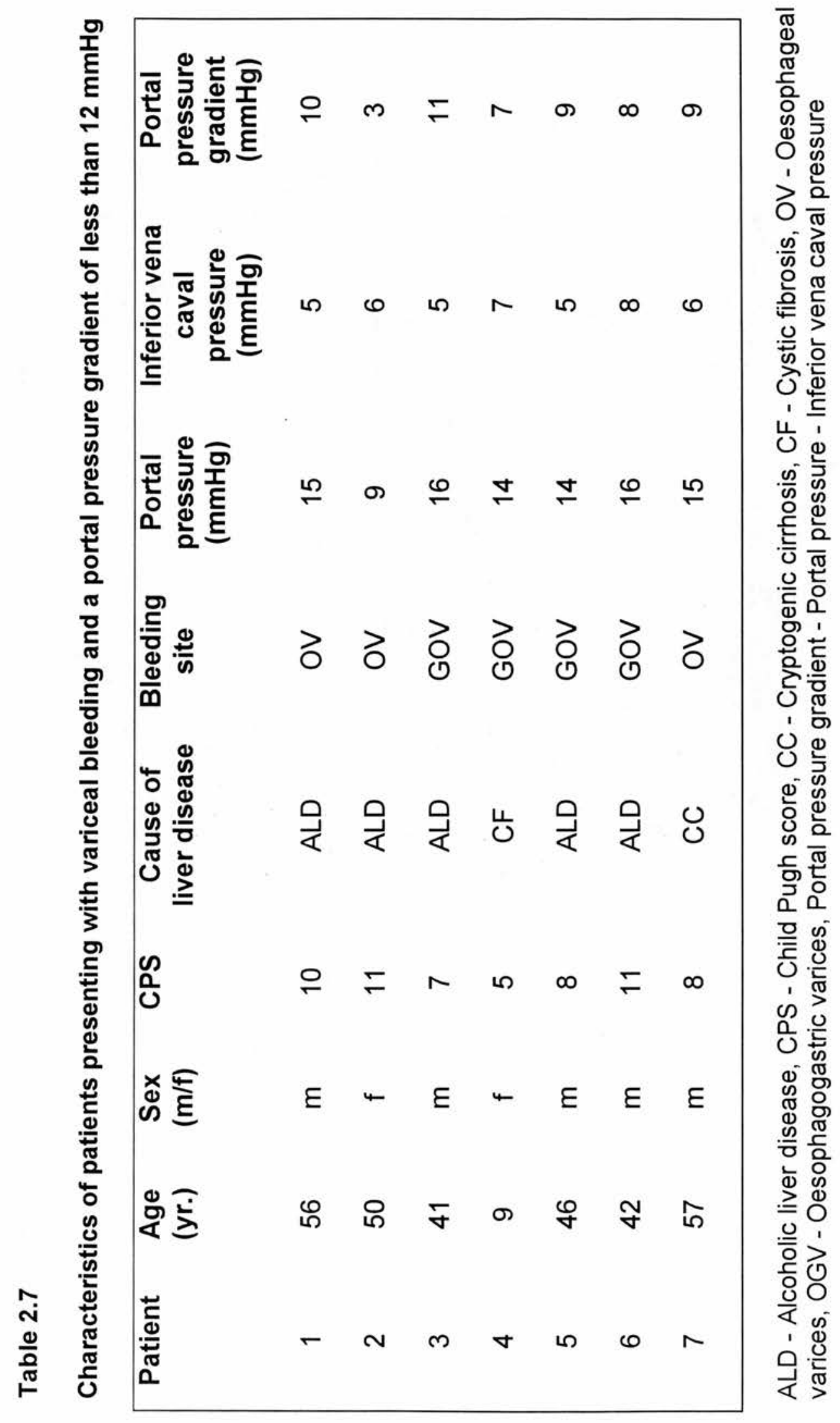




\subsection{Increase in intracranial pressure after TIPSS}

Acute liver failure (ALF) is defined by a rapidly developing hepatic encephalopathy in the presence of acute liver injury of less than 8 weeks duration. This is often progressive over a period of days or even hours and is characterised neuropathologically by cerebral oedema. There is a high associated mortality if left untreated, with up to $40 \%$ of deaths in patients with ALF, resulting from the effects of increased intracranial pressure and reduced cerebral perfusion pressure (Jones EA and Schafer DF, 1990). Chronic hepatic encephalopathy (HE) is a complex neuropsychiatric syndrome, typified by global depression of the central nervous system (CNS) with varying degrees of severity. However, it is a reversible metabolic abnormality, the manifestations of which are widely variable and involve a spectrum from mild subclinical disturbance to deep coma.

About $15-20 \%$ patients become encephalopathic after TIPSS. Although numerous studies have shown a transient deterioration in liver function after TIPSS, features of ALF are not a well recognised complication following TIPSS placement. Four patients developed features of 'acute liver failure' with hypoglycemia and elevation in the intracranial pressure. Patient characteristics are summarised in Table 2.8.

\section{Case 1}

This 55 year old man with alcoholic cirrhosis and Child class C (Pugh 12) disease presented with severe ascites, grade II HE and oesophageal variceal haemorrhage which was controlled after two sessions of sclerotherapy. He required mechanical ventilation for aspiration pneumonia. Five days later he 
rebled and TIPSS was performed as an emergency. He developed epileptic seizures after TIPSS and became hypoglycaemic. A CT scan showed cerebral oedema. Mannitol infusion was started and an intracranial pressure (ICP) monitor inserted. This showed an initial ICP of $13 \mathrm{mmHg}$ which rapidly increased to $26 \mathrm{mmHg}$ and then to $37 \mathrm{mmHg}$. Thiopental infusion was started with some initial improvement but neurological deterioration continued and the patient died 3 days later with cerebral oedema. Changes in liver function tests are illustrated in Table 2.9.

\section{Case 2}

This 69 year old lady with primary biliary cirrhosis and Child class C (Pugh 10) disease presented with grade $1 \mathrm{HE}$, mild ascites and variceal bleeding. After resuscitation she was endoscoped which showed bleeding from gastric varices. Bleeding was controlled using a Sengstaken tube and TIPSS was performed as an emergency. The following day she became profoundly encephalopathic and had epileptic seizures. She was mechanically ventilated and CT scan of brain showed diffuse brain oedema. Mannitol was infused and an intracranial pressure monitor inserted which showed an initial ICP of $16 \mathrm{mmHg}$ which rose to $22 \mathrm{mmHg}$ and finally to $34 \mathrm{mmHg}$. The jugular bulb oxygen saturation was measured at $65 \%$. ICP was controlled initially using mannitol and thiopentone infusion but she died of progressive liver failure and cerebral oedema 3 days after insertion of TIPSS. Changes in her liver function tests are illustrated in Table 2.9.

Case 3 
This 74 year old lady had cryptogenic cirrhosis and Child class B (Pugh 8) presented with mild ascites, grade $1 \mathrm{HE}$ and bleeding oesophageal varices. Bleeding was controlled with endoscopic variceal sclerotherapy. The following day she rebled and became profoundly hypotensive from which she was resuscitated successfully. She was mechanically ventilated and an endoscopy showed bleeding varices which continued despite sclerotherapy. Bleeding was controlled with Sengstaken tube and TIPSS was performed as an emergency. The following day the pupils were poorly reactive and an ICP monitor was inserted which showed an ICP of $24 \mathrm{mmHg}$. This was poorly controlled despite mannitol and thiopentone infusion. Subsequently, she developed hypoglycaemia, epileptic seizures and acidosis. Her ICP rose to $34 \mathrm{mmHg}$ and then to $41 \mathrm{mmHg}$. She died the day after the TIPSS procedure. Changes in her liver function tests are illustrated in Table 2.9.

\section{Case 4}

This 42 year old man with alcoholic cirrhosis and Child class C (Pugh 10) disease presented with mild ascites, grade $1 \mathrm{HE}$ and variceal haemorrhage. Following resuscitation, an endoscopy confirmed bleeding from oesophageal varices which was not controlled with sclerotherapy. A Sengstaken tube was inserted and TIPSS performed. Two hours after insertion of TIPSS the patient developed seizures and was electively ventilated. Mannitol infusion was started and an ICP monitored inserted. ICP was $27 \mathrm{mmHg}$ which rose to 30. The ICP was successfully controlled with Mannitol and thiopentone infusion. Despite an ICP of $3 \mathrm{mmHg}$ the patient developed fixed and dilated pupils and died. Changes in his liver function tests are illustrated in Table 2.9. 
The mechanism of development of cerebral oedema in these patients can be explained on the basis of some innovative studies by Cordoba et.al. (1995). They showed in a rat model that there was a significant and progressive reduction in brain myoinositol when they were subjected to portacaval anastomosis. When ammonia was infused in large doses there was a significant increase in brain water even when accompanied by reduced myoinositol. The introduction of TIPSS is like producing a side-to-side portacaval shunt which may provide a large dose of ammonia particularly if the patients were actually bleeding at the time of TIPSS. Furthermore, in a recent study, they also showed that the reduction in myoinositol does not offer protection against a new osmotic stimulus, i.e ammonia or hyponatremia. All 4 of these patients exhibited hyponatremia at the time of insertion of TIPSS and this may have sensitised them to the development of increased ICP. Hyponatremia is an independent marker of poor outcome in patients undergoing TIPSS for variceal haemorrhage (Jalan et al. 1995).

These cases suggest that cerebral oedema and an increase in ICP may occur in patients with cirrhosis having TIPSS for uncontrolled variceal haemorrhage. 


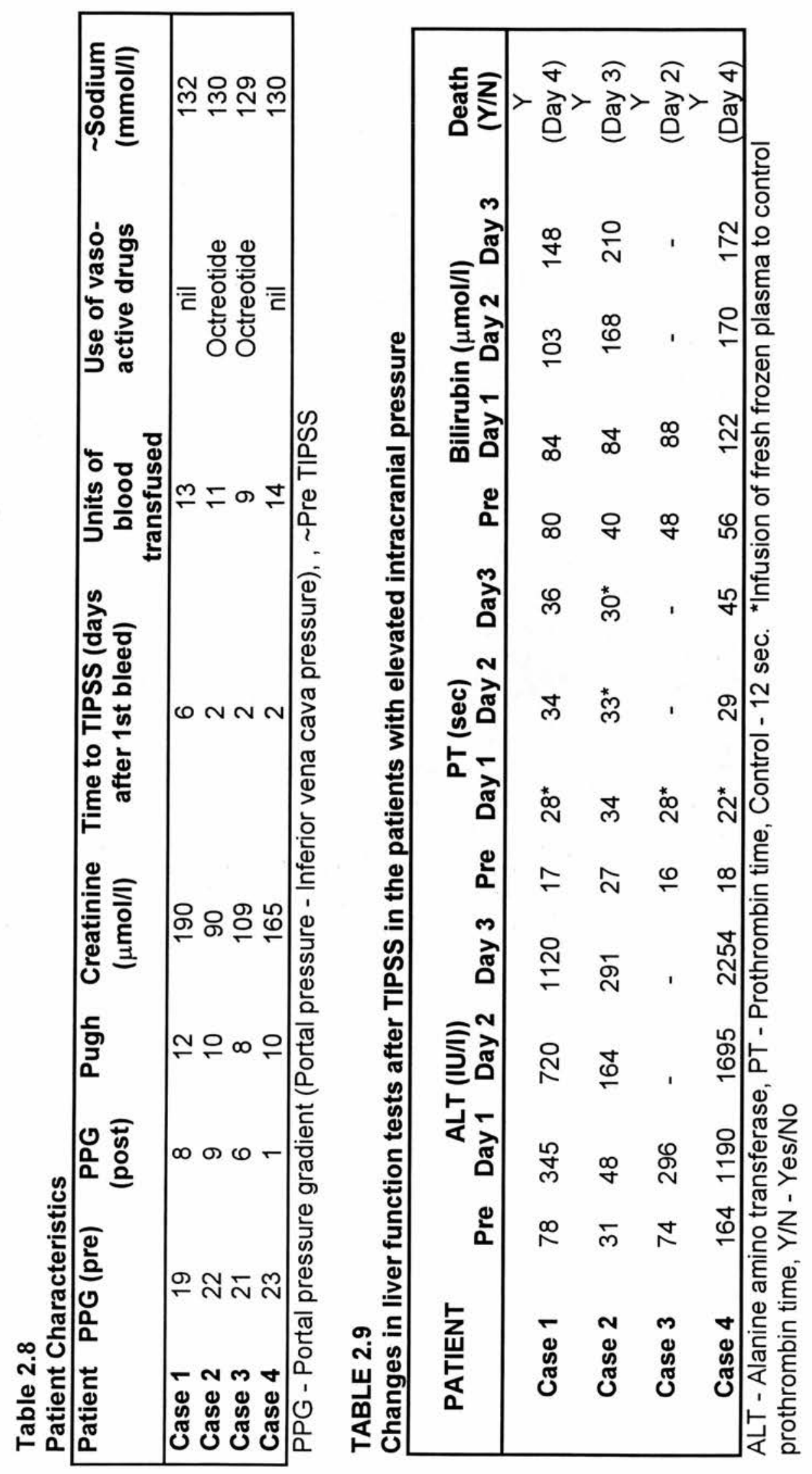




\subsection{Changes in Neuropsychological and Liver function}

\section{Clinical}

No significant complications of the procedure were encountered during or immediately after TIPSS. During follow up, 4 patients developed significant shunt dysfunction (PPG $>12 \mathrm{mmHg}$ or an increase in the PPG by more than $20 \%$ over the value attained following TIPSS). Two presented with variceal haemorrhage within 7 days of TIPSS and one with re accumulation of ascites about 3 weeks after TIPSS. The other patient was diagnosed on routine screening at 1 month. They were all managed successfully with balloon angioplasty or insertion of a parallel shunt. Overall, the PPG (Figure 2.11) did not change significantly during follow up.

Overt encephalopathy was precipitated immediately following TIPSS in one patient. This failed to respond to medical therapy and was only poorly controlled following reduction in the diameter of the shunt. This patient underwent orthotopic liver transplantation (OLT). Four other patients died during follow up from complications of end stage liver failure and an additional patient underwent liver transplantation.

Neuropsychological function, $H A D$ and $Q O L$

Patients with cirrhosis both in the TIPSS and the banding groups performed significantly worse on all three of the subtests of CANTAB and RBMT. Ten of the 15 patients in the TIPSS group who were recruited into the study prior to TIPSS $(67.6 \%)$ had values of simple and complex reaction times that fell outside 2 standard deviations of the normal. Delayed matching to sample 


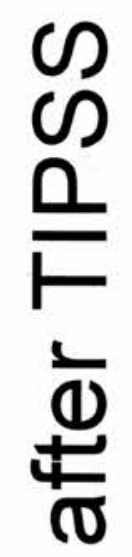

(1)

믐

.

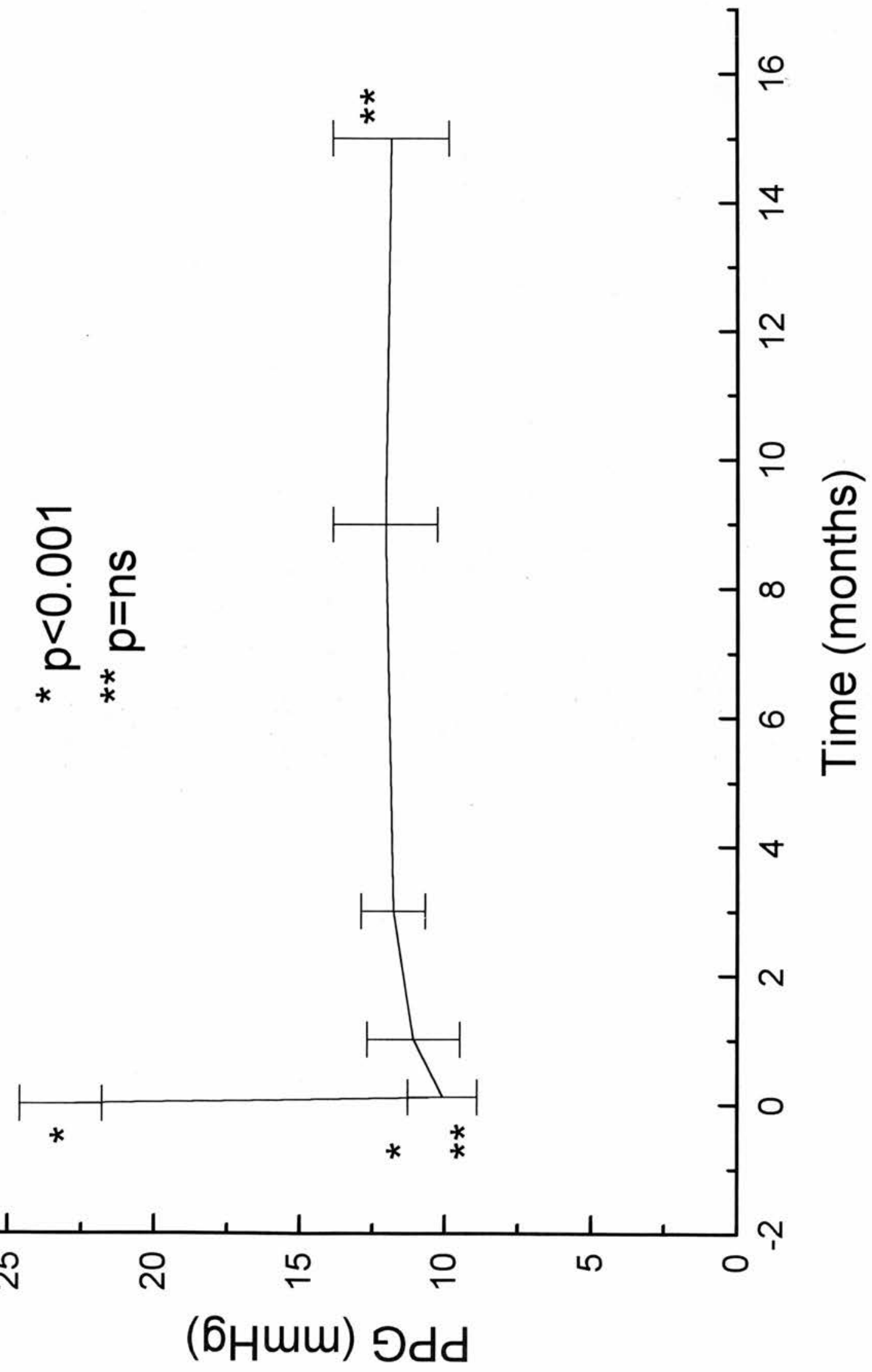

$\frac{\text { O }}{\frac{1}{\sigma}}$ 
W

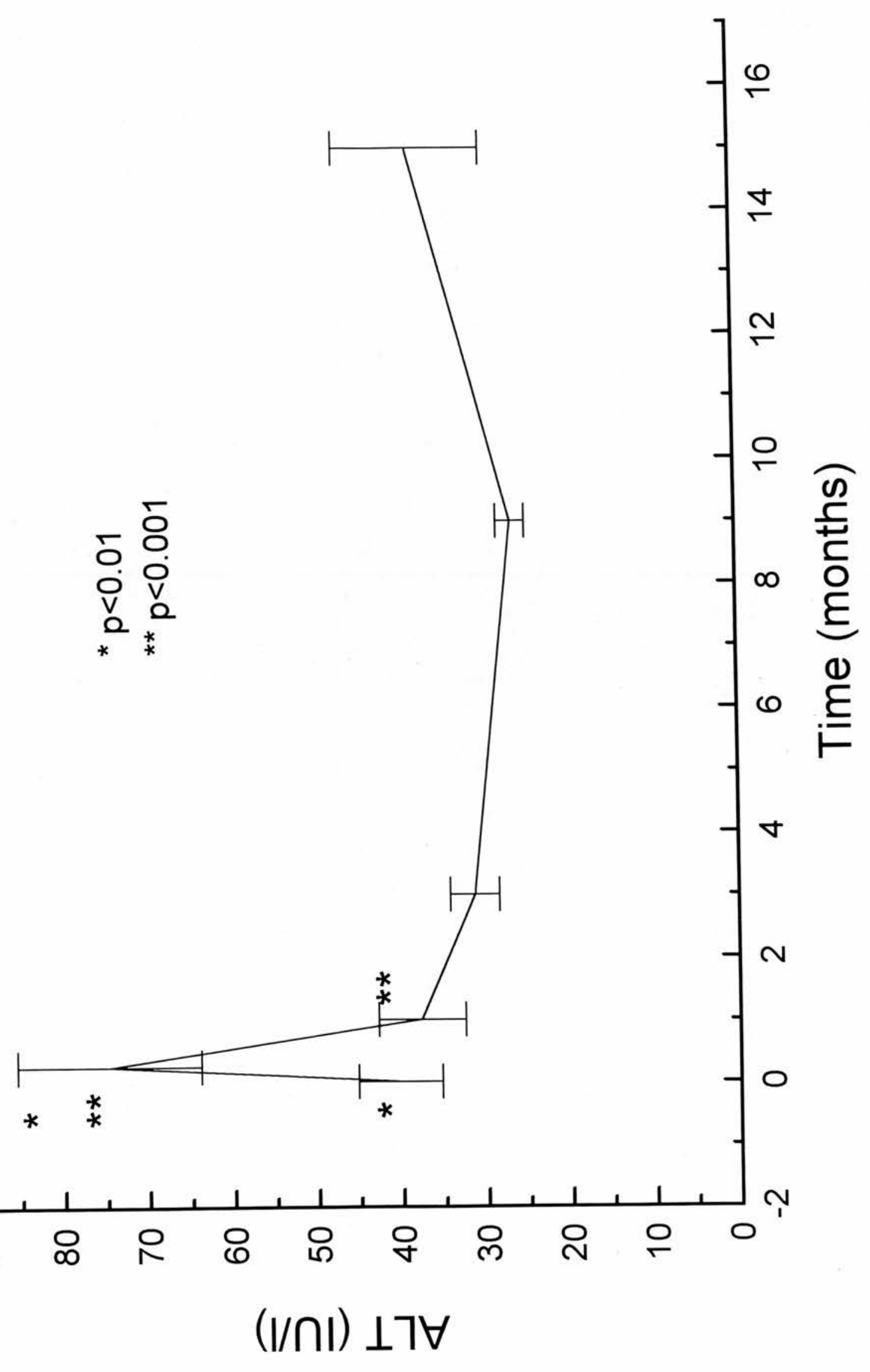


(S)

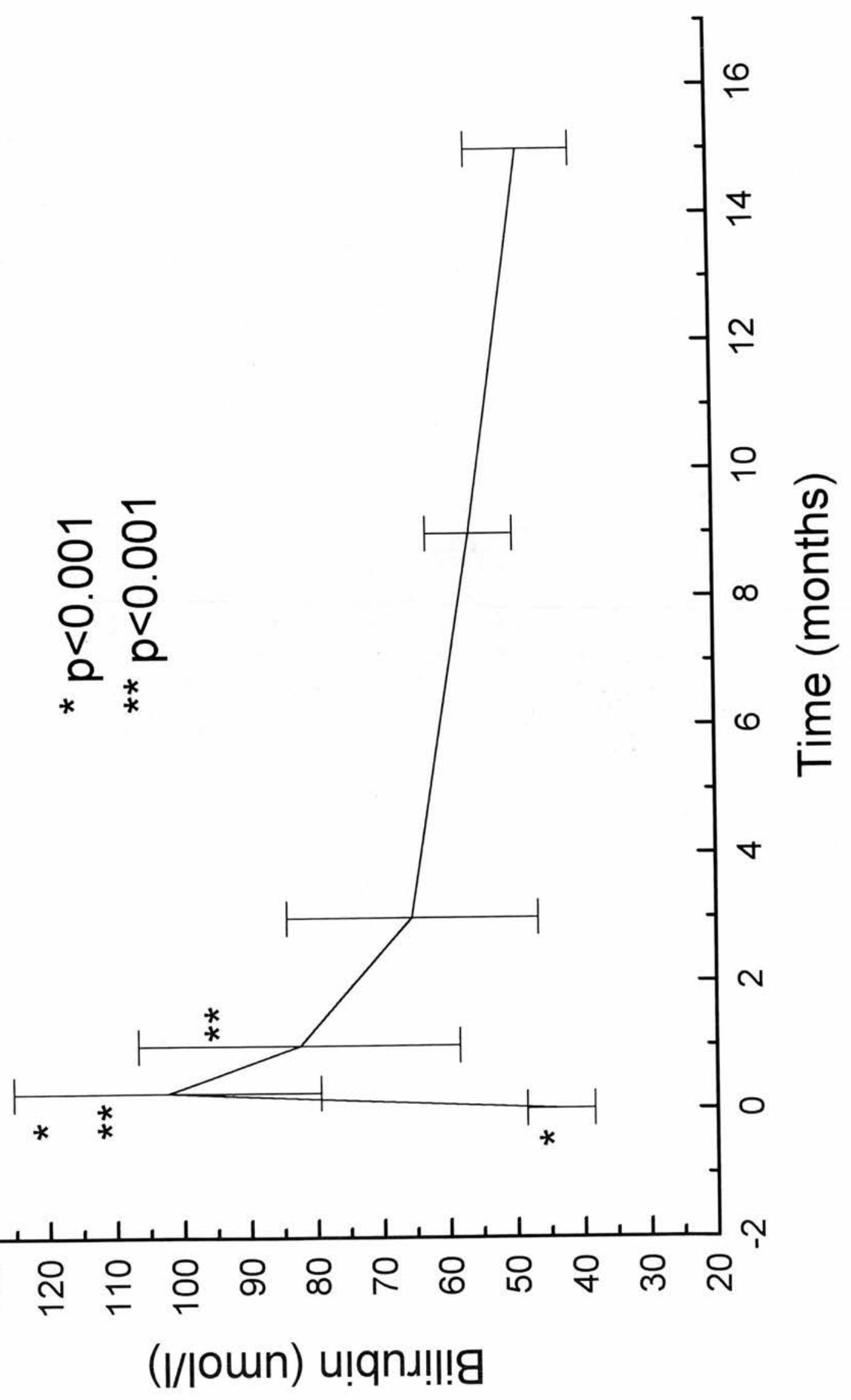




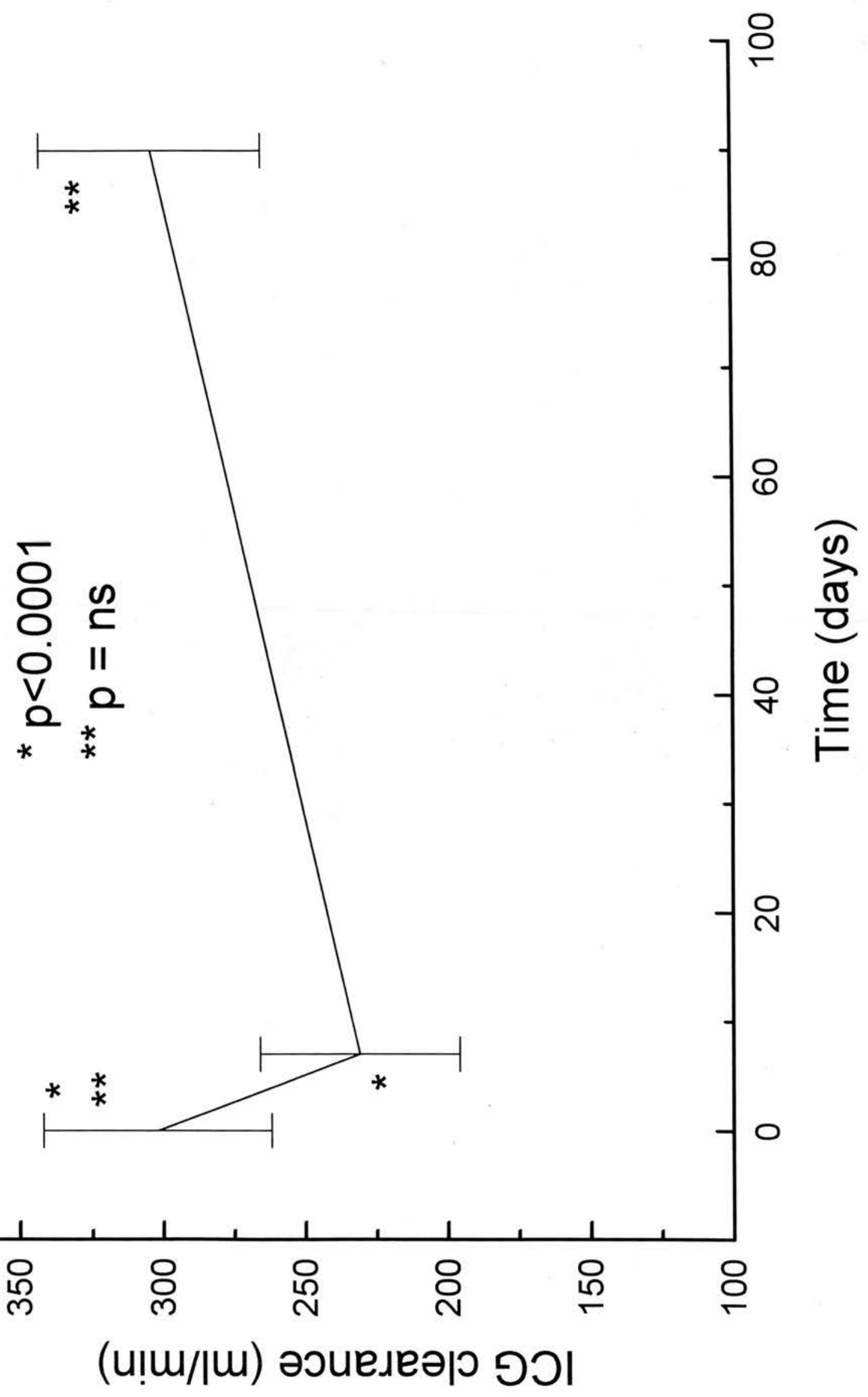




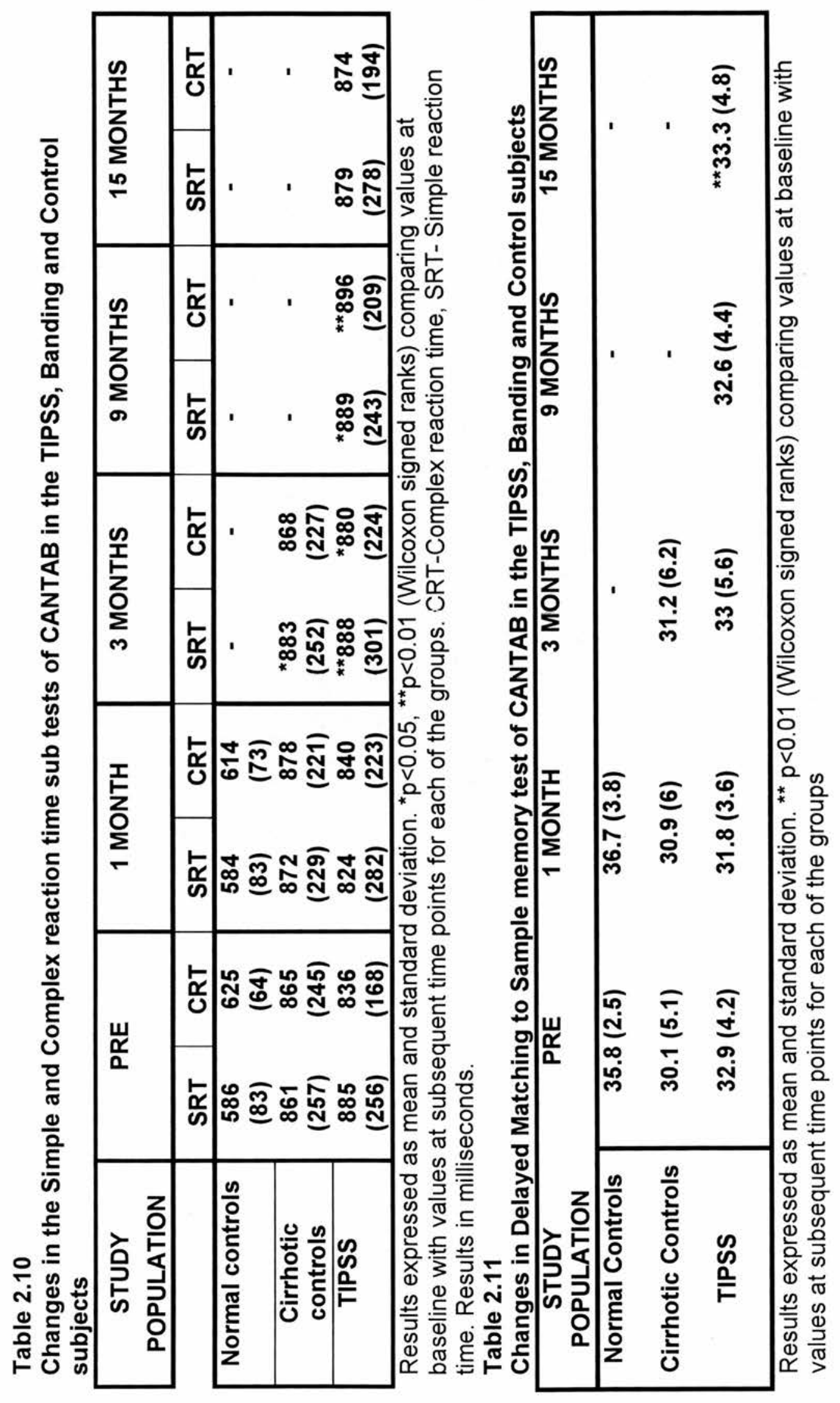




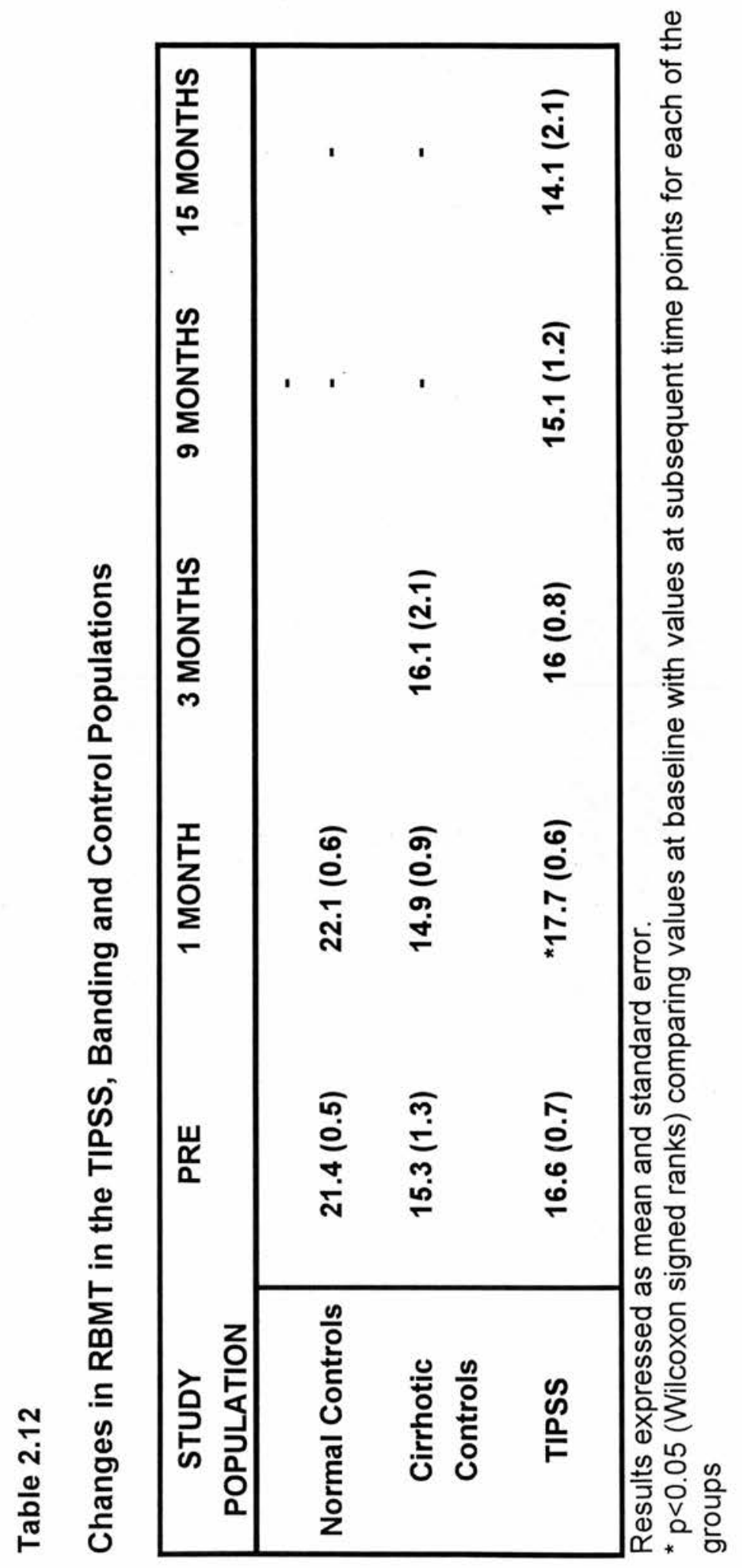


Patients in both the TIPSS and banding groups had significantly higher HAD scores $(p<0.05$ and $p<0.01$ respectively) compared with the control group. There were no significant differences in the HAD score between the TIPSS and banding groups. Levels of anxiety following TIPSS improved significantly at 1 month but deteriorated subsequently. Levels of anxiety were significantly lower in the normal volunteers at 1 month. No significant change was seen in the patients in the banding group (Table 2.13).

Patients in the TIPSS group had significantly worse quality of life on both physical $(p<0.001)$ and psychological $(p<0.01)$ measures when compared with normal volunteers. Similarly, patients in the banding group had significantly poorer scores for $Q O L(p<0.01$ and $p<0.05$ respectively for physical and psychological components). There was no significant difference in the quality of life between patients in the TIPSS and the banding groups. Psychological component of the quality of life deteriorated significantly in the patients in the TIPSS group. No significant change was noted in the patients in the banding group or normal controls (Table 2.14). 


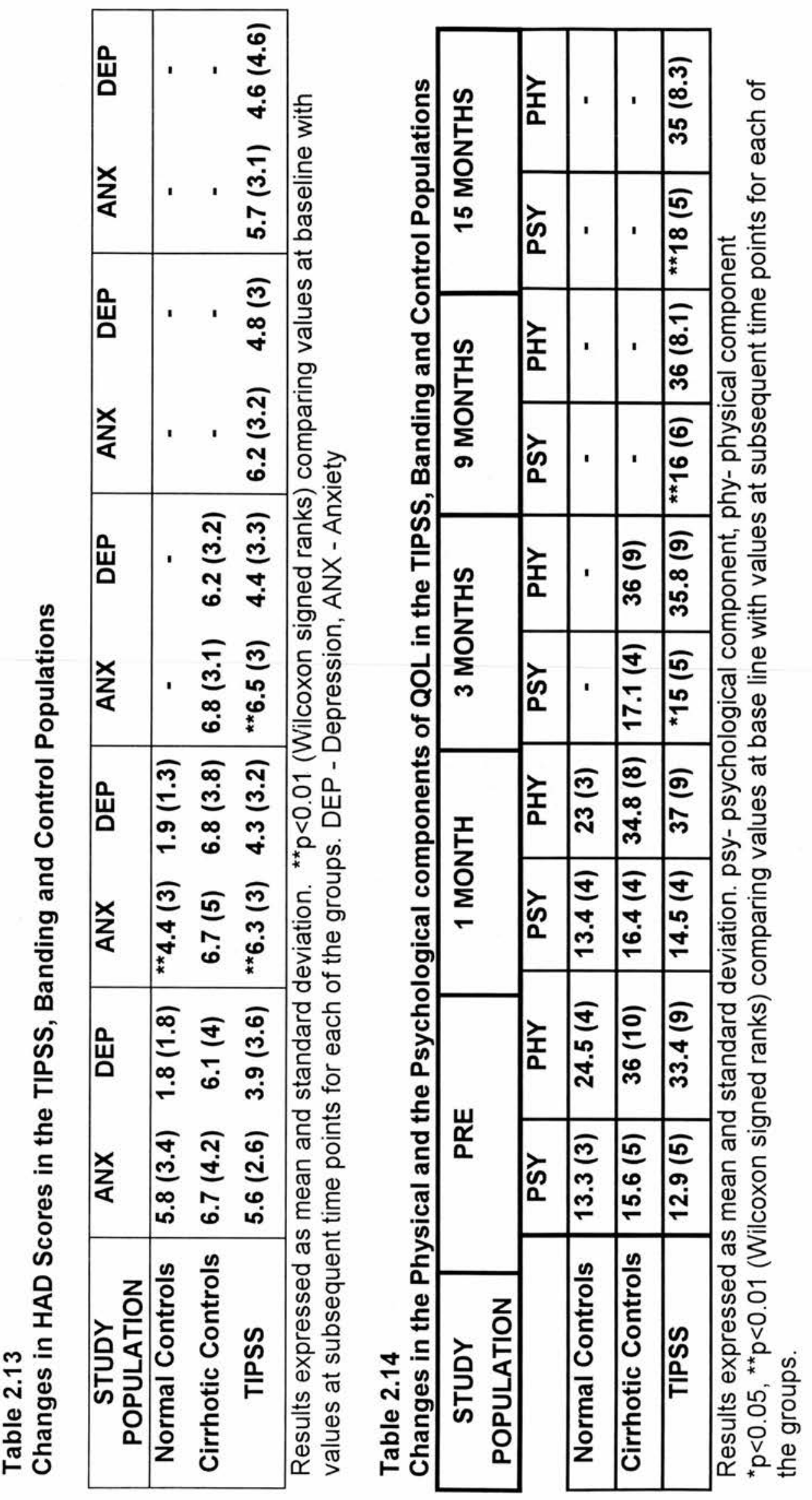




\section{Liver function tests}

Serum alanine transaminase and bilirubin deteriorated significantly following TIPSS (Figure $2.12,2.13$ ) but both returned to the pre-TIPSS value during follow up. There was no significant change in serum albumin or prothrombin time following TIPSS. No significant change was noted in the above parameters during follow up in the patients in the banding group. There was dramatic reduction in ICG clearance following TIPSS which returned to pre TIPSS values during follow up (Fig. 2.14).

Good correlation was observed between the changes in ICG clearance and the change in serum bilirubin and ALT $(p<0.01 r=0.71$, and $p<0.01, r=.68$ respectively) when measured 7 days following TIPSS Although, the changes in ICG clearance seemed to parallel the changes in PPG no significant correlation was observed between these parameters $(r=$ $0.3)$

Relationship between changes in the neuropsychological tests and liver function tests and $P P G$

Changes in the neuropsychological tests were completely independent of changes in the conventional liver function tests. There was however, good correlation between changes in both the complex and simple reaction times (sub tests of CANTAB) and the changes in the ICG clearance ( $r=0.6, p, 0.05$, and $r=0.66, p<0.05$, respectively) measured at 3 months. No correlation was observed between changes in the PPG and the changes in neuropsychological function. 


\subsection{Results of Haematological assessment}

\section{Clinical}

TIPSS was performed successfully with an immediate reduction in the portal pressure gradient from a mean of $21.9( \pm 1.6)$ to $9.5( \pm 1.0)(p<0.01)$. There was no significant change in the pressure thereafter. Three patients did however, develop significant shunt insufficiency which was manifested by variceal rebleeding in 2 and reaccumulation of ascites in 1 . Three patients died during follow up from their underlying, end stage chronic liver disease and 1 underwent successful orthotopic liver transplantation after 4 months. The patients have been followed up for a mean of 8.0 months $( \pm 1.2)$ (longest 19 months).

Haematology

Haemoglobin concentration improved significantly from 89.9 (se 1.6) g/l to 119 (1.9) $\mathrm{g} / \mathrm{l}$ after TIPSS ( $<<0.05)$ (Fig 2.16). No evidence of haemolysis was detected prior to TIPSS with negative or normal Coombs test, reticulocyte counts, serum haptoglobin , undetectable urinary haemosiderin and absence of fragmented red cells in the peripheral blood. There was no significant change in the mean reticulocyte count, mean cell volume or serum haptoglobin level (Table 2.15). During the first week following TIPSS 3 patients (13\%) showed evidence of haemolysis manifested by increase in the reticulocyte count to 7,8 . and $10 \%$ respectively with a simultaneous reduction in the haemoglobin concentration requiring at least 2 units of blood transfusion, without any signs of bleeding (Fig 2.17). This was also associated with reduction in serum haptoglobin to undetectable levels and 
the appearance of haemosiderin in the urine. Fragmented red cells suggestive of traumatic haemolysis were also present on the peripheral blood film. These features of haemolysis disappeared within 2 weeks of the TIPSS and there was no evidence of haemolysis at 3 months. Four other patients developed transient haemolysis, which was not accompanied by a change in the haemoglobin concentration and associated with the appearance of fragmented red cells and the disappearance of measurable haptoglobin from the serum.

There was a significant reduction in the size of the spleen from a mean of $16.9( \pm 1.1)$ to $13.7( \pm 2.4) \mathrm{cm}(\mathrm{p}<0.01)$ (Figure 2.18$)$ at 3 months. No significant change occurred thereafter. There was neither any significant correlation between changes in the portal pressure gradient and the change in spleen size $(r=0.3)$ nor was there a correlation between these parameters prior to TIPSS $(r=0.12)$.

The platelet count changed significantly following TIPSS $(p<0.01)$ (Figure 2.19) but no significant correlation was observed between changes in the portal pressure gradient and platelet count $(r=0.38)$. No significant correlation existed between them prior to TIPSS $(r=0.15)$. There was no correlation between the change in the size of the spleen and change in the platelet count $(r=0.29)$. White cell count did not change significantly (3.6 to $\left.4.1 \times 10^{9} / l\right)$. 


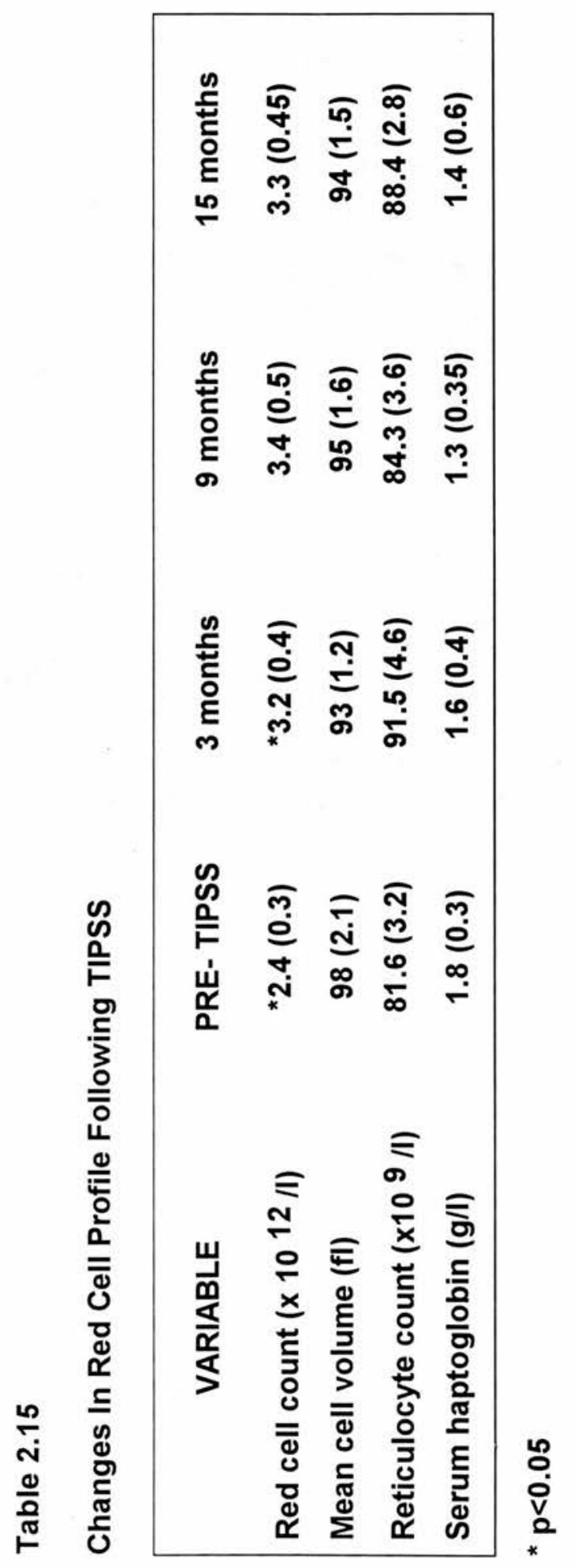




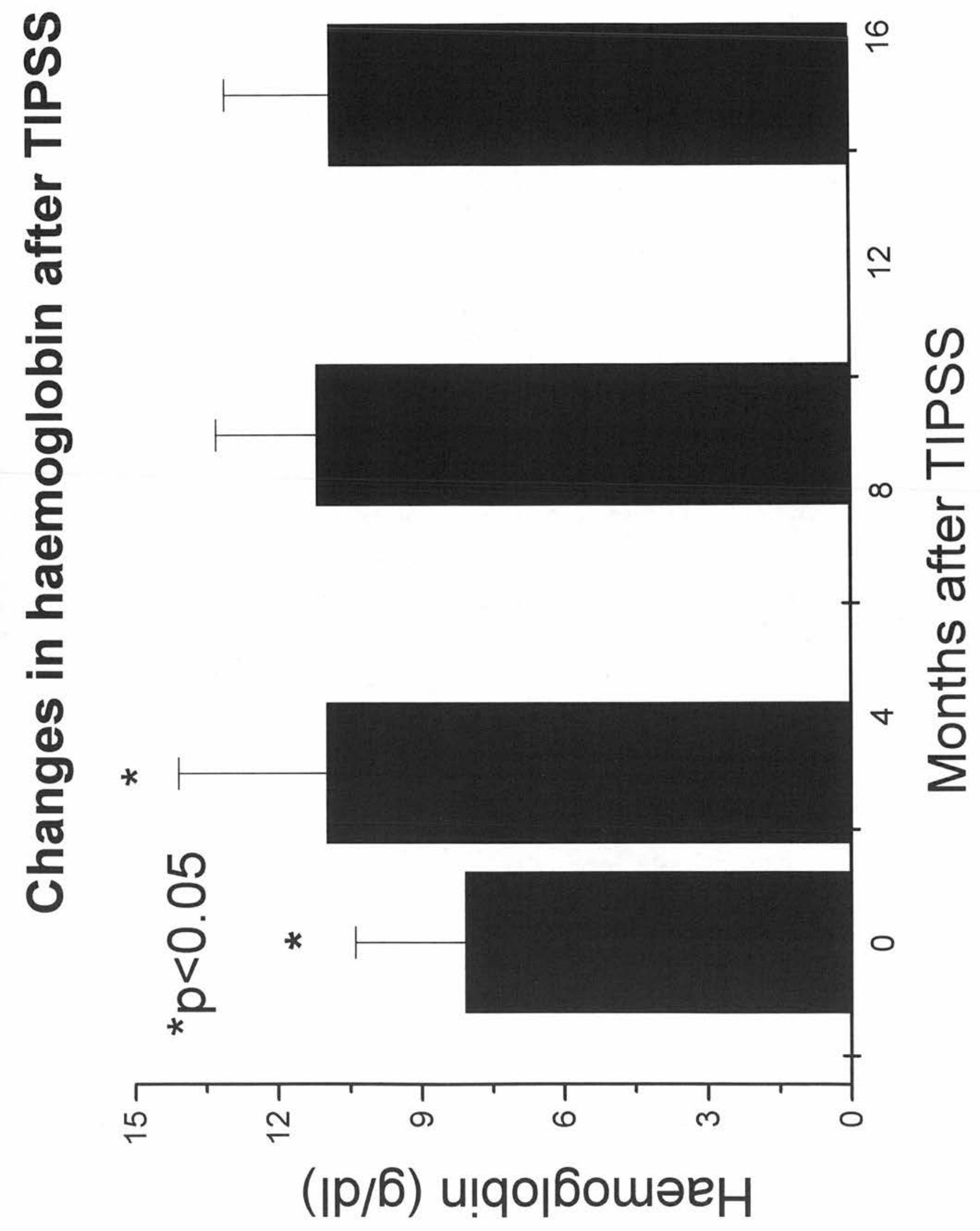




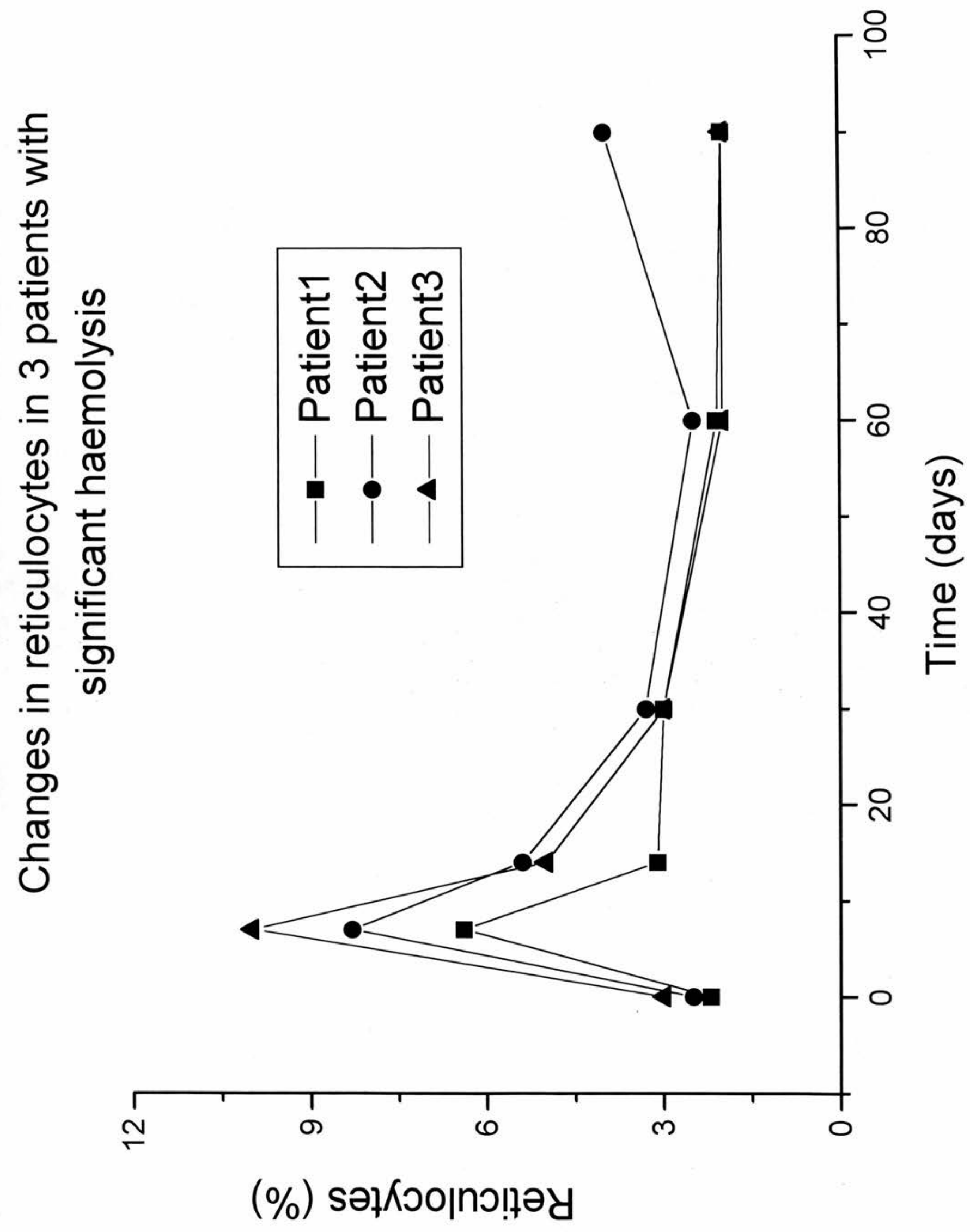


Spleen size $(\mathrm{cm})$

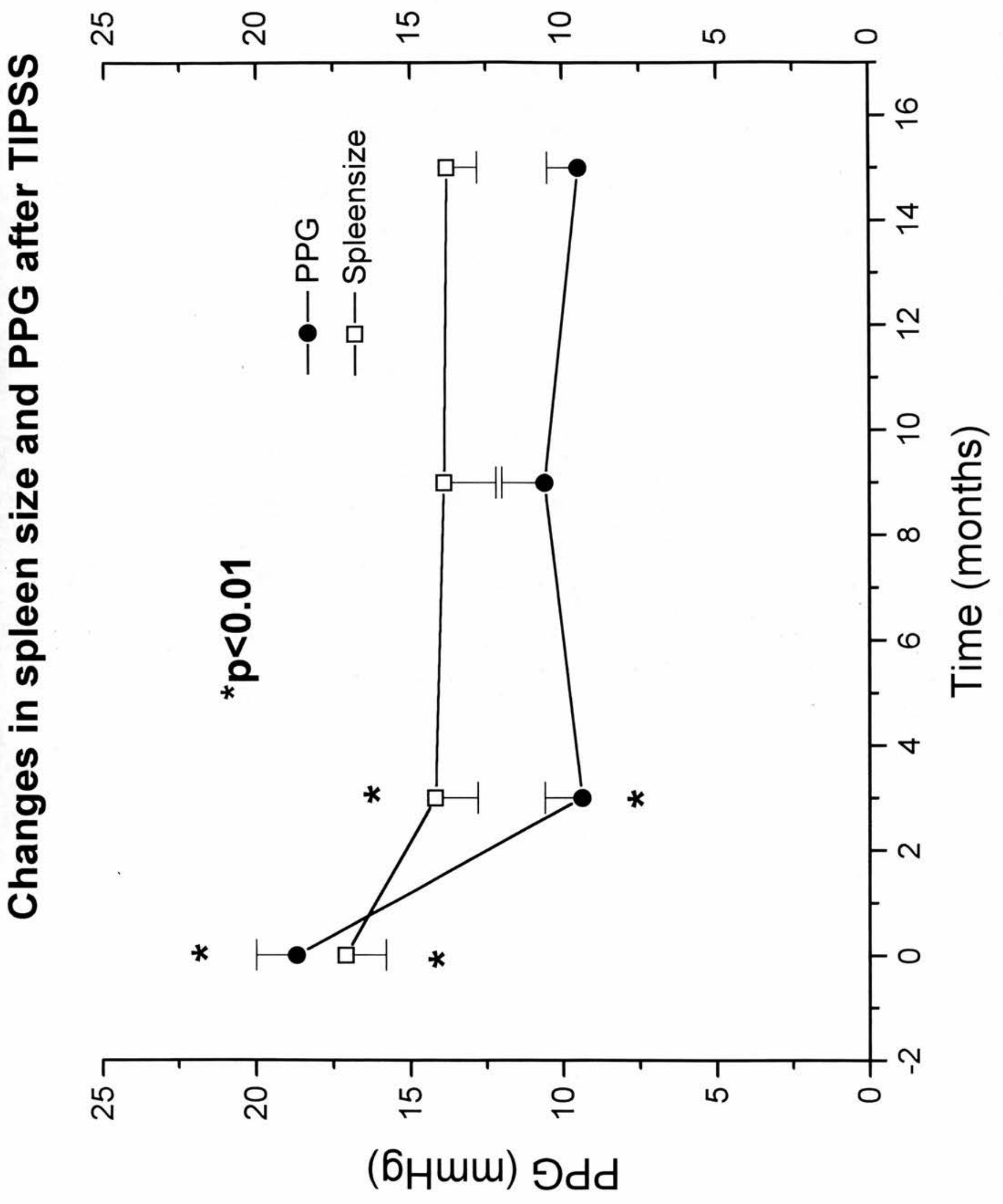




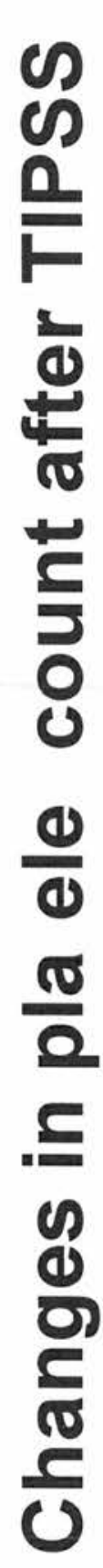

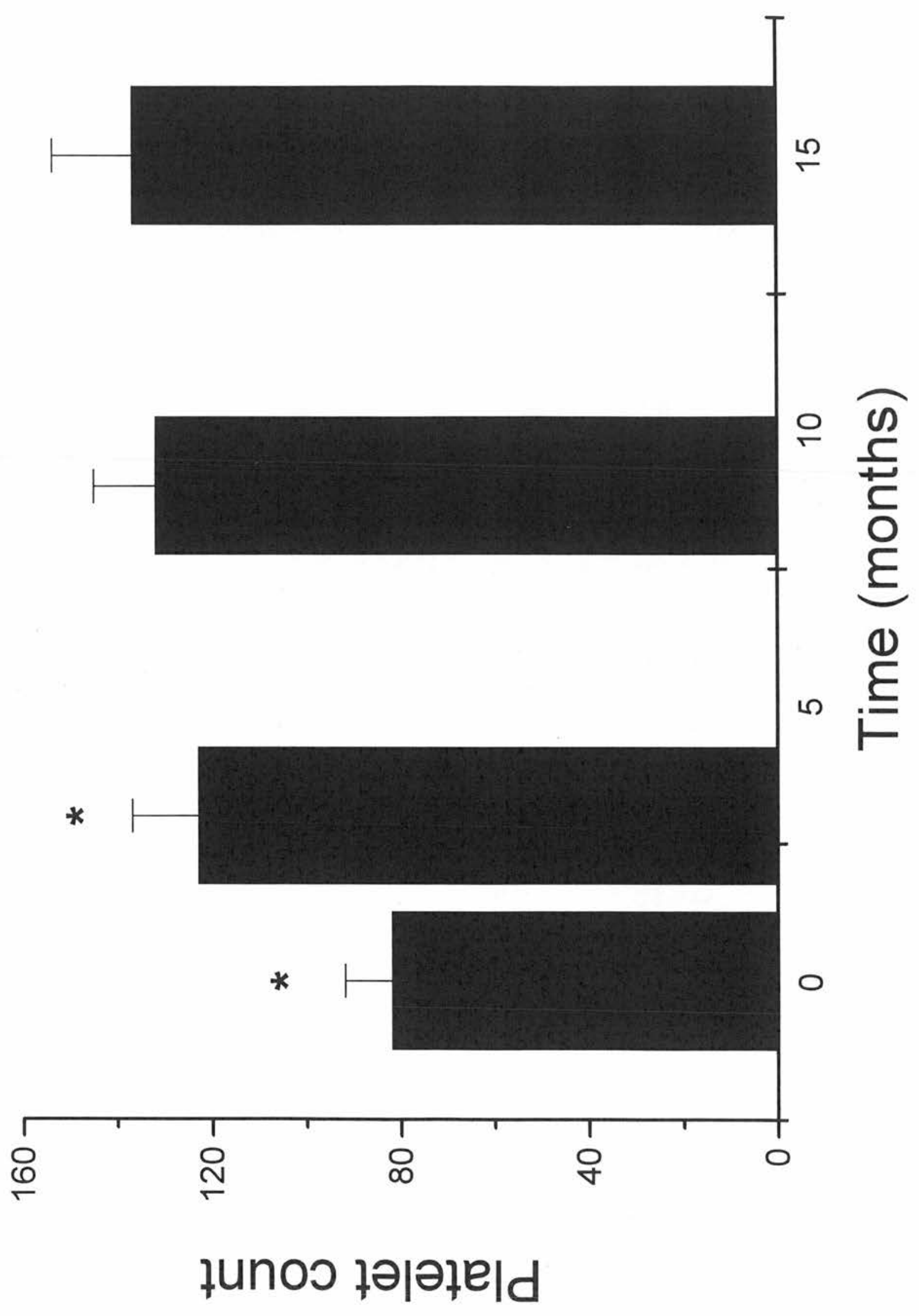


1. TIPSS is relatively safe and can be performed on very ill patients with a high degree of success in the management of bleeding varices. Numerous questions remain unanswered such as which patients should be offered this treatment, what is the complication rate and how best to monitor these patients. It is, however, clear that complications are frequent and usually associated with shunt insufficiency and regular and detailed follow up is required to identify and address these at an early stage.

2. We have found that severe liver disease and hyponatremia indicate a poor outlook after TIPSS and a high portal pressure predicts shunt failure. The precipitation of encephalopathy following TIPSS was predicted independently only by its presence prior to TIPSS. These predictors should lead to consideration of the value of the procedure, the need for anticipation of shunt failure and rebleeding and perhaps the use of prophylactic measures such as lactulose and smaller shunts in patients likely to develop encephalopathy.

3. The commonly accepted dictum that a PPG of $>12 \mathrm{mmHg}$ is required for variceal haemorrhage requires revision. The relationship between variceal rupture, its relationship to portal pressure and splanchnic blood flow clearly require further study. 
4. The development of cerebral oedema and an increase in ICP after TIPSS for uncontrolled variceal haemorrhage is of importance both from the point of view of clinical practice and from the point of view of understanding the pathogenesis of cerebral oedema in patients with liver disease. All 4 of our patients exhibited hyponatremia at the time of insertion of TIPSS and this may have sensitised them to the development of increased ICP.

5. (a) Clinical encephalopathy in this selected group of patients with moderately severe liver disease occurred in 3\% patients following TIPSS. Neuropsychological tests were significantly worse in cirrhotic patients compared with controls, $67 \%$ showing evidence of subclinical encephalopathy. In this group of patients without previous encephalopathy there was a temporary deterioration in the neuropsychological function both in patients having TIPSS and the cirrhotic controls.

(b) Although temporary deterioration occurred in the levels of anxiety and the psychological component of the quality of life in patients with TIPSS, measures of memory improved. Significant deterioration occurred in ALT, bilirubin and ICG clearance.

(c) Changes in ICG clearance following TIPSS correlated significantly with the changes in the neuropsychological tests. Further studies should address the mechanism of these changes and the effect of different prophylactic measures on their prevention. 
6. Clinically significant haemolysis occurs in a minority of patients and is mild and transient. TIPSS is associated with reduction in splenomegaly and improves red cell and platelet counts and may therefore be useful for troublesome hypersplenism. Further studies should address specific changes in the functional aspects of the various haematological parameters following TIPSS. 


\section{CHAPTER 3}

\section{SHUNT DYSFUNCTION}

- Methods Of Follow Up

- Mechanisms Of Shunt Stenosis and Occlusion 


\subsection{BACKGROUND AND AIMS}

The aim of follow-up is to identify the TIPSS patients with shunt insufficiency and re-intervene before variceal bleeding recurs. The "gold standard" is venous portography via the shunt with measurement of the PPG. This is invasive and costly compared with clinical assessment or ultrasound examination. Clinical assessment and endoscopic findings are poor predictors of shunt dysfunction. Ideally non-invasive ultrasound screening for shunt dysfunction would allow regular follow-up. Portography and re-intervention would then only be required when shunt stenosis or occlusion was suspected or identified.

The aims of this prospective study was to assess Duplex and Colour Doppler ultrasonography in the assessment of shunt function and compare the results with portography and PPG measurement in patients following successful TIPSS. 


\subsection{METHODS}

\subsection{Study Design}

Shunt function was assessed routinely 3 months after insertion. None of the patients showed clinical evidence of shunt dysfunction such as acute or chronic gastro-intestinal blood loss or ascites at the time of follow-up. Shunt function was assessed using Duplex and Colour flow Doppler within 24 hours of portography and measurement of PPG. The investigators performing the portographic examination and the ultrasonography were blinded to one another's results and also to the clinical details.

\section{Patients}

Twenty three patients ( 15 male, 8 female) with a mean age of 53 years were assessed with both Duplex and Colour Doppler and portography in the followup of TIPSS. The indication for TIPSS was variceal haemorrhage in all patients. Fifteen patients had alcoholic cirrhosis, 4 patients had cryptogenic cirrhosis, 2 patients had Hepatitis B associated cirrhosis and 1 patient each had autoimmune chronic active hepatitis and Hepatitis $C$ virus infection each. Ten patients were Child-Pugh class A, 8 class $B$, and 7 class $C$ at presentation. TIPSS were created using the Wallstent (Schneider (Europe) AG, Switzerland) in a similar manner to that previously reported (Jalan et.al. 1994).

\subsection{Portography And Measurement Of Portal Pressure}

Details of the method used for portography are described in section 3.21. 


\subsection{Doppler Ultrasonography}

Duplex and colour Doppler sonography were carried out by a single experienced operator (Dr PL Allan) using an Acuson 128/XP (Acuson Corp., Mountain View, Calif., U.S.A.) with a $3.5 \mathrm{MHz}$ OR $4 \mathrm{MHz}$ phased-array transducers. Values in triplicate were obtained for peak velocity, time averaged velocity, cross-sectional area and from this blood flow and congestive index were calculated (Moriyasu et.al. 1986) for both the main portal vein and the shunt.

\section{Analysis}

Adequate Doppler examination was not possible in 6 patients due to their large body habitus. Mean Doppler values for the previously defined variables were calculated on each of the remaining 17 patients and correlated with the PPG using linear regression. To demonstrate the range of results these have been expressed as a mean and standard error for each variable (Table 3.1). 
Table 3.1

\begin{tabular}{|ll|}
\hline Measured Variable & Result \\
Shunt peak velocity $(\mathrm{cm} / \mathrm{sec})$ & $90.6( \pm 12)$ \\
Shunt time averaged velocity $(\mathrm{cm} / \mathrm{sec})$ & $40.6( \pm 7)$ \\
Shunt cross sectional area $\left(\mathrm{cm}^{2}\right)$ & $1.0( \pm 0.1)$ \\
Portal vein peak velocity $(\mathrm{cm} / \mathrm{sec})$ & $68.4( \pm 7)$ \\
Portal vein time averaged mean velocity $(\mathrm{cm} / \mathrm{sec})$ & $21.4( \pm 3)$ \\
Portal vein cross-sectional area $\left(\mathrm{cm}^{2}\right)$ & $2( \pm 0.1)$
\end{tabular}




\subsection{RESULTS}

\subsection{Patients}

Shunt function was assessed routinely 3 months after insertion. None of the patients showed clinical evidence of shunt dysfunction such as acute or chronic gastro-intestinal blood loss or ascites at the time of follow-up. Patients were also assessed clinically for signs of hepatic encephalopathy both prior to and following TIPSS. Two patients without prior encephalopathy became clinically encephalopathic.

\subsection{Portography, Doppler And Correlation}

There was no significant correlation between portal vein peak velocity (PVPV), portal vein blood flow (PVBF), portal vein congestive index (PVCl), shunt blood flow (SBF), shunt congestive index (SCl) and the PPG. All 7 patients $(41 \%)$ with a shunt peak velocity (SPV) $>90 \mathrm{~cm} / \mathrm{sec}$ had normal shunt function at portography (PPG $<12 \mathrm{~mm} \mathrm{Hg}$ ). However, a SPV $<90 \mathrm{~cm} / \mathrm{sec}$ was not predictive of the measured PPG (Figure 3.1). Shunt insufficiency at portography (PPG $\geq 12$ ) was diagnosed in 8 patients $(47 \%)$, due to intimal hyperplasia in 6 and hepatic vein stenosis in 2 .

In the two patients who developed hepatic encephalopathy following TIPSS the SBF:PVBF ratio on Doppler ultrasound was $>1.0$ and $<1.0$ on all the other patients. 

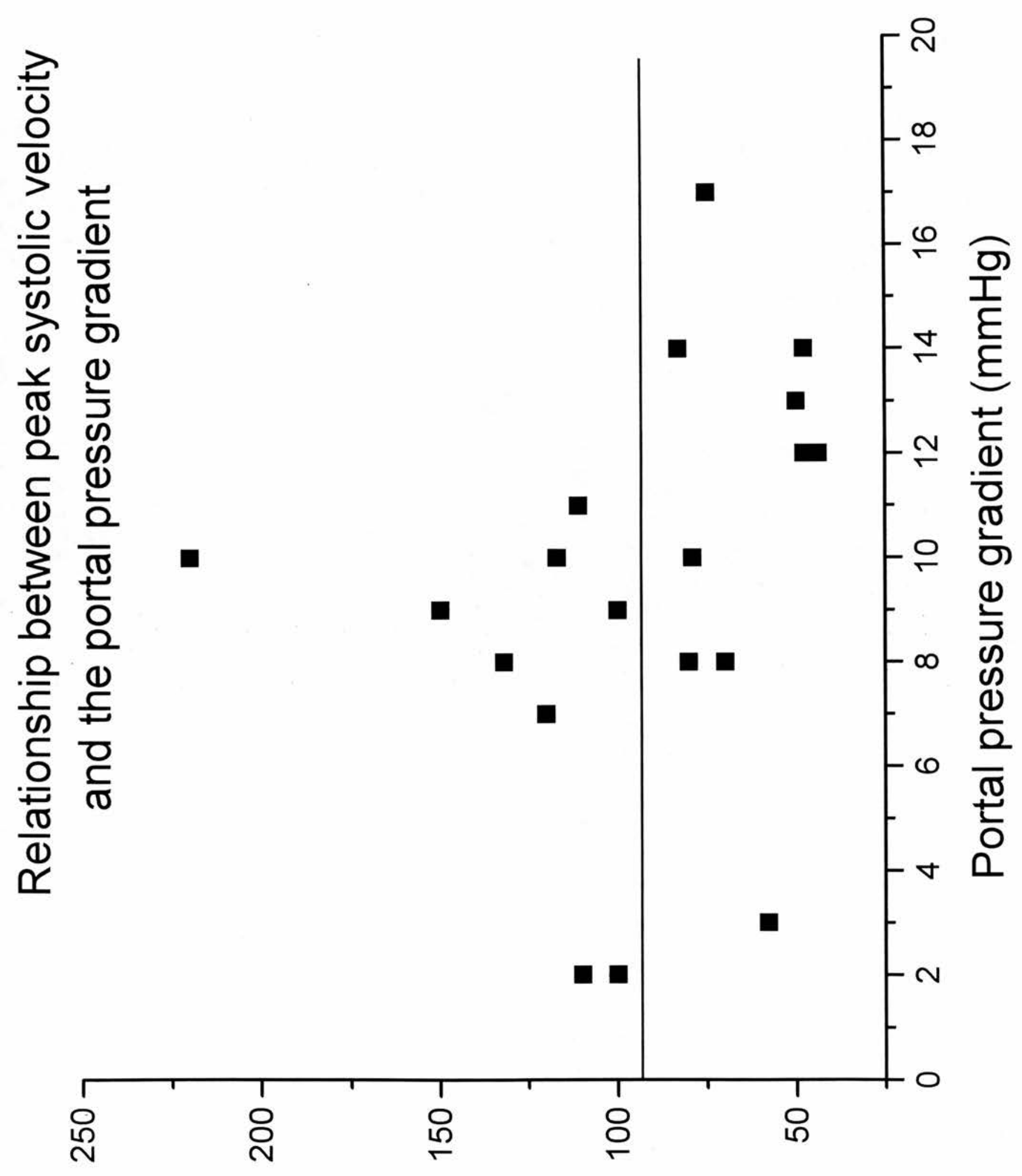

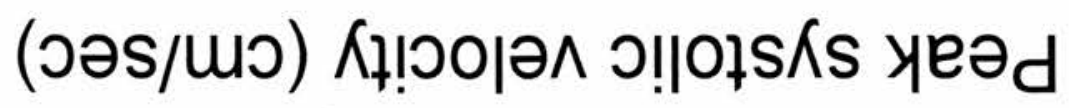


This study assessing Doppler ultrasound in the assessment of shunt function shows that a peak velocity in the shunt $>90 \mathrm{~cm} / \mathrm{sec}$ indicates that the PPG is < $12 \mathrm{~mm} \mathrm{Hg}$. SPV may be high at about $200 \mathrm{~cm} / \mathrm{sec}$ in a functioning shunt (Foshager et.al 1994) but localised values $>200 \mathrm{~cm} / \mathrm{sec}$ may be due to a focal stenosis associated with a raised PPG. The lack of correlation of blood flow and PPG is probably related to the difficulties of accurate measurement of area within the shunt. The shunt often lies deep within the abdomen and visualisation of the whole shunt and the hepatic vein is difficult. Furthermore, stenoses are often poorly visualised and the cross-sectional area if measured between the metal stent struts will not reflect the true luminal area when intimal hyperplasia is present.

An interesting observation was the association in 2 patients of significant hepatic encephalopathy with a measurable ratio of SBF to PVBF of $>1.0$. Larger studies would be required to confirm the validity of this observation. The presumed reason for this high ratio is that shunt blood is derived from not only the main portal vein but the hepatic artery via shunting through the hepatic portal vein branches which flow retrogradely to the shunt. These patients have too large a shunt and some form of shunt reduction may need to be undertaken such as inserting a stent with a smaller diameter within the existing shunt.

Our results therefore show sonographic evaluation of TIPSS follow-up is of only limited value. The finding of an SPV $>90 \mathrm{~cm} / \mathrm{sec}$ but $<200 \mathrm{~cm} / \mathrm{sec}$, particularly with maintenance of reversed portal vein branch flow, indicates a 
functioning shunt and satisfactory PPG. However, the finding of a SPV of $<90$ $\mathrm{cm} / \mathrm{sec}$ may not indicate shunt malfunction and portography is required for further evaluation. This probably reflects inaccurate angle correction when the shunt is difficult to visualise clearly at a favorable Doppler angle which has been noted by others authors (Longo et.al. 1993).

It is concluded that portography remains the "gold standard" for accurate TIPSS follow-up assessment and is still required in the majority of patients. However, if the Doppler criteria of SPV $>90 \mathrm{~cm} / \mathrm{sec}$ without a focal increase in SPV $>200 \mathrm{~cm} / \mathrm{sec}$ is met, then portography may not be required. 
3.2 INVESTIGATION INTO THE PATHOGENESIS OF SHUNT INSUFFICIENCY AFTER TIPSS

\subsection{INTRODUCTION AND AIMS}

The pathology and pathogenesis of shunt stenosis or occlusion is unclear and differs according to when this occurs and whether it is due to narrowing at the venous end or within the shunt. Radiologically, shunt insufficiency may be due to narrowing of the hepatic vein (hepatic vein stenosis) or due to stenosis or occlusion within the shunt itself. Narrowing of the portal vein is not commonly seen (Darcy et.al. 1992). Early shunt occlusion is invariably associated with thrombosis of the shunt, whilst late dysfunction is due to hepatic vein stenosis or stenosis within the shunt due to "pseudo intimal hyperplasia" (Ring et.al. 1992, LaBerge et.al. 1993).

The pathogenesis of hepatic vein stenosis is not clear although intimal hyperplasia due to exaggerated proliferative response from local trauma and stent motion, and retraction of the Wallstent following full expansion have been blamed (Haskal et.al. 1994, Lind et.al. 1994).

The aim of this study was to assess the pathology and pathogenic mechanisms of stenosis and occlusion within the shunt. 


\subsection{METHODS}

\subsection{Patients and clinical follow up}

The study population comprised 34 patients who had TIPSS inserted for variceal haemorrhage (29), ascites (3) and portal gastropathy (2), and had at least 2 separate portographic assessments of shunt function. Mean follow up was 8.3 months $( \pm 1.2)$ (range 1-32 months). Patient characteristics are summarised in Table 3.2 .

Table 3.2.

Patient characteristics

\begin{tabular}{|ll|}
\hline Variable & $\mathbf{n = 3 4}$ \\
& \\
Age (mean \pm se) & $56( \pm 1.6)$ \\
Sex (M/F) & $20 / 14$ \\
Aetiology of Liver disease & \\
& \\
Alcoholic cirrhosis & 27 \\
Cryptogenic cirrhosis & 04 \\
Primary biliary cirrhosis & 01 \\
Hepatitis B/C & $1 / 1$ \\
& \\
Child class & \\
& \\
Class A & 09 \\
Class B & 17 \\
Class C & 08 \\
Indication for TIPSS & \\
& \\
Variceal haemorrhage & 29 \\
Refractory ascites & 03 \\
portal gastropathy & 02 \\
Duration of follow up & \\
\hline
\end{tabular}

Results expressed as mean \pm se

\section{TIPSS Procedure}


TIPSS was performed by the standard procedure and has been described in detail in section 2.21 .

\section{Follow Up}

These patients were followed up clinically, biochemically and with Doppler ultrasonography at 3 month intervals. In all patients, portography for assessment of shunt function was carried out routinely at 3 and 6 months and 6 monthly thereafter or earlier if they developed complications. Shunt insufficiency was defined as either an increase in the porta-caval gradient to greater than $12 \mathrm{mmHg}$ or portographic evidence of shunt insufficiency $(>70 \%)$. Balloon dilatation was performed when shunt insufficiency was diagnosed either portographically or manometrically. Parallel stents were inserted in 3 patients with recurrent intraparenchymal shunt insufficiency.

\subsection{Pathology}

\section{Shunt Biopsy}

In cases of shunt stenosis diagnosed at portography, endoscopic biopsy forceps (Olympus, FB24K) were introduced through the sheath into the stent and 4 biopsies of the occluding material taken from within the shunt (Figure 3.2). These were fixed in $4 \%$ formaldehyde and processed routinely.

Sections were cut at 4 microns and stained with haematoxylin/eosin, PAS, PAS-diastase, Prussian blue reaction and Van Gieson for elastic tissue. Immunohistochemistry was performed using PKK1/CAM 5.2 for epithelial 
Fig 3.2 Radiograph showing endocopy biopsy forceps introduced into the shunt through the sheath for obtaining biopsies of the occluding material.

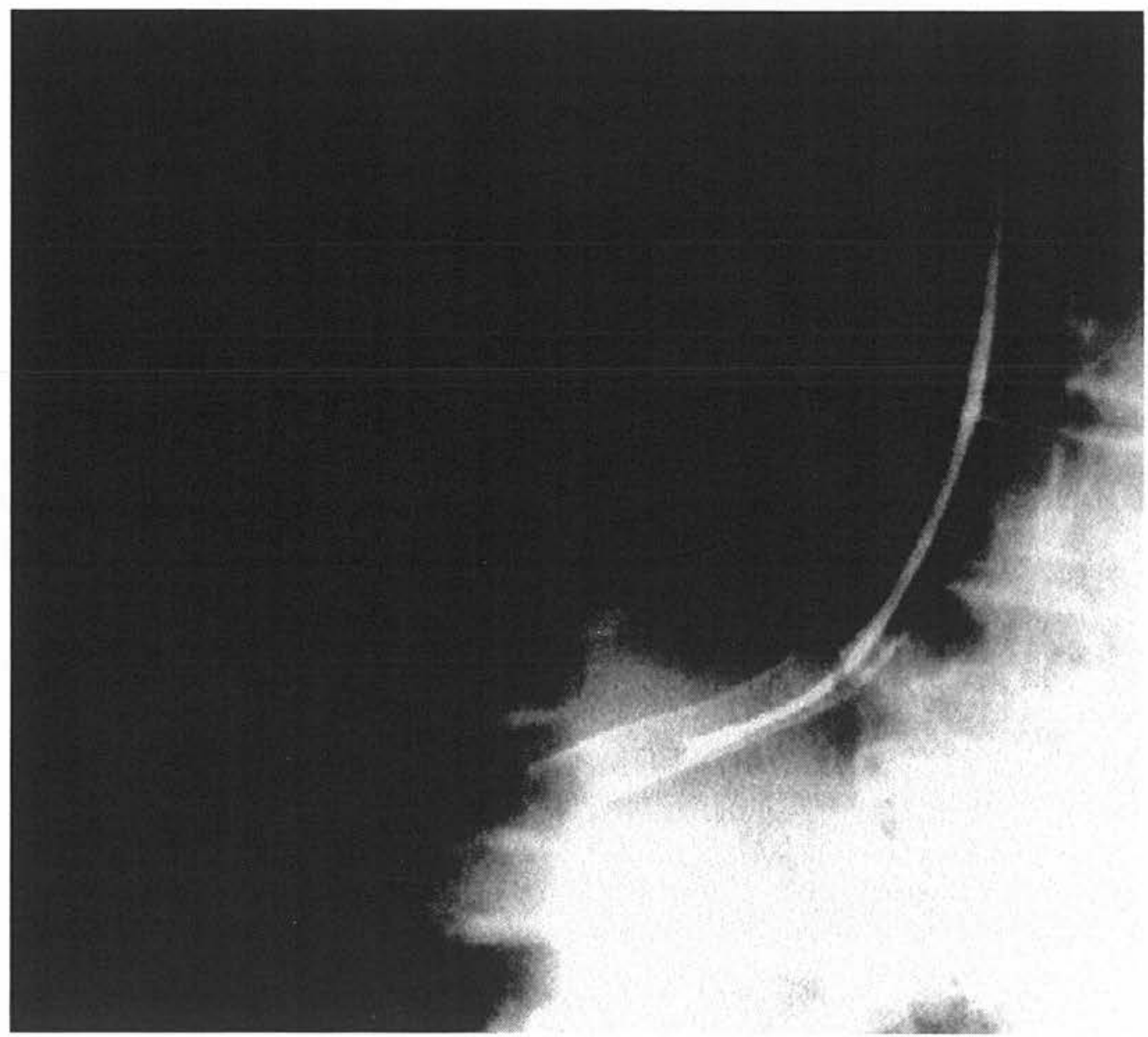


cells, factor V111 for endothelial cells (Dako Itd., U.K.) using standard avidin biotin - peroxidase detection with diaminobenzidine as substrate.

\subsection{Explant Livers}

Livers were obtained from the 10 patients who either died (8) or underwent orthotopic liver transplantation (2). Only 1 of these patients had instrumentation to the TIPSS within 30 days. The mean duration from insertion of the TIPSS to obtaining the liver was 5.6 months (range 1-13 months). They were processed for radiological and pathological examination as described below.

Pathology: The liver was perfused and fixed in buffered formalin immediately afterwards and dissected to remove the stent with approximately $2 \mathrm{~cm}$ of the surrounding liver. Several sections (at $5 \mathrm{~mm}$ intervals) were taken from the surrounding liver. The stent was then opened with wire cutters. The contents were examined macroscopically, the lining tissue was stripped off with forceps and the contents taken separately. The tissues were processed as described previously.

Radiology: All these explanted livers were placed on ice and the common bile duct cannulated using a 10 Fr. Foley's catheter. Under radiological screening, contrast was injected to fill the right and left biliary ductal systems. Radiographs were taken at various angles to outline the ductal anatomy especially in the vicinity of the shunt. 


\subsection{RESULTS}

\subsection{Clinical}

Successful control of bleeding and ascites was achieved in all the patients in whom the TIPSS was inserted successfully. Requirements for blood transfusion were significantly reduced in patients who had TIPSS inserted for portal gastropathy. During the study 8 patients have died and 2 have been transplanted.

Depending upon the results of the first portographic assessment and subsequent follow up the patients could be clearly divided into 3 groups There was no significant difference in the post-TIPSS porta-caval gradient in the three groups. Group A (19 patients) comprised the patients in whom no shunt insufficiency was noted. Group B (6 patients) comprised the patients with mild shunt stenosis $(<30 \%$ and a porta-caval gradient $<12 \mathrm{mmHg}$ ) and Group C (8 patients) was composed of patients with severe shunt stenosis ( $>70 \%$ and a porta-caval gradient of $>12 \mathrm{mmHg}$ ). Three patients in Group C presented with variceal rebleeding whilst the remainder were discovered on routine screening. None of these patients had any evidence of hepatic vein stenosis.

During follow up there was no significant change in the porta-caval gradient in the patients in Group A and B (Figures 3.3 and 3.4). Shunt dysfunction in patients in Group C was initially managed by balloon dilatation and this normalised the haemodynamic abnormality at the time but re stenosis occurred by the time of the second assessment in all. Shunt 
occlusion in this group was manifested by variceal rebleeding in 2 patients and re-accumulation of ascites in 2 . The other 4 patients with severe shunt stenosis were symptomless and identified only on routine portography. Three of the 4 patients with complications were managed by insertion of parallel stents through another hepatic vein (Figure 3.5) and remained free of shunt dysfunction on subsequent assessment (Figure 3.6). The other 5 patients who were treated by dilatation had significant shunt stenosis at subsequent portography (Figure 3.7).

\subsection{Radiology}

Direct portography in 1 patient prior to the biopsy showed a persistent biliary venous fistula in association with shunt stenosis when contrast was injected forcefully into the shunt (Figure 3.8). Cholangiography in the post mortem livers showed marked distortion of the biliary tree around the shunt indicating fibrous cicatrization (Fig 3.9). Although, 3 of these patients had severe shunt stenosis and macroscopic evidence of transection of a major bile duct, persistence of a patent fistulous connection could not be demonstrated due possibly to thrombosis of the transected bile duct.

\subsection{Pathology:}

4.2331. Shunt biopsy Adequate biopsy specimens were obtained in all with severe stenosis. In patients with minor stenosis $(<30 \%)$ the biopsies were too poor to be adequately examined. This was due to paucity of tissue and the degree of fibrosis of the underlying pseudo intima. Organised thrombus 


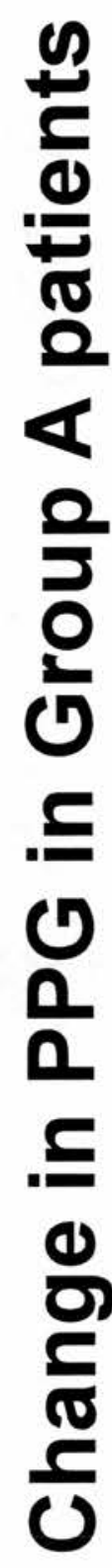

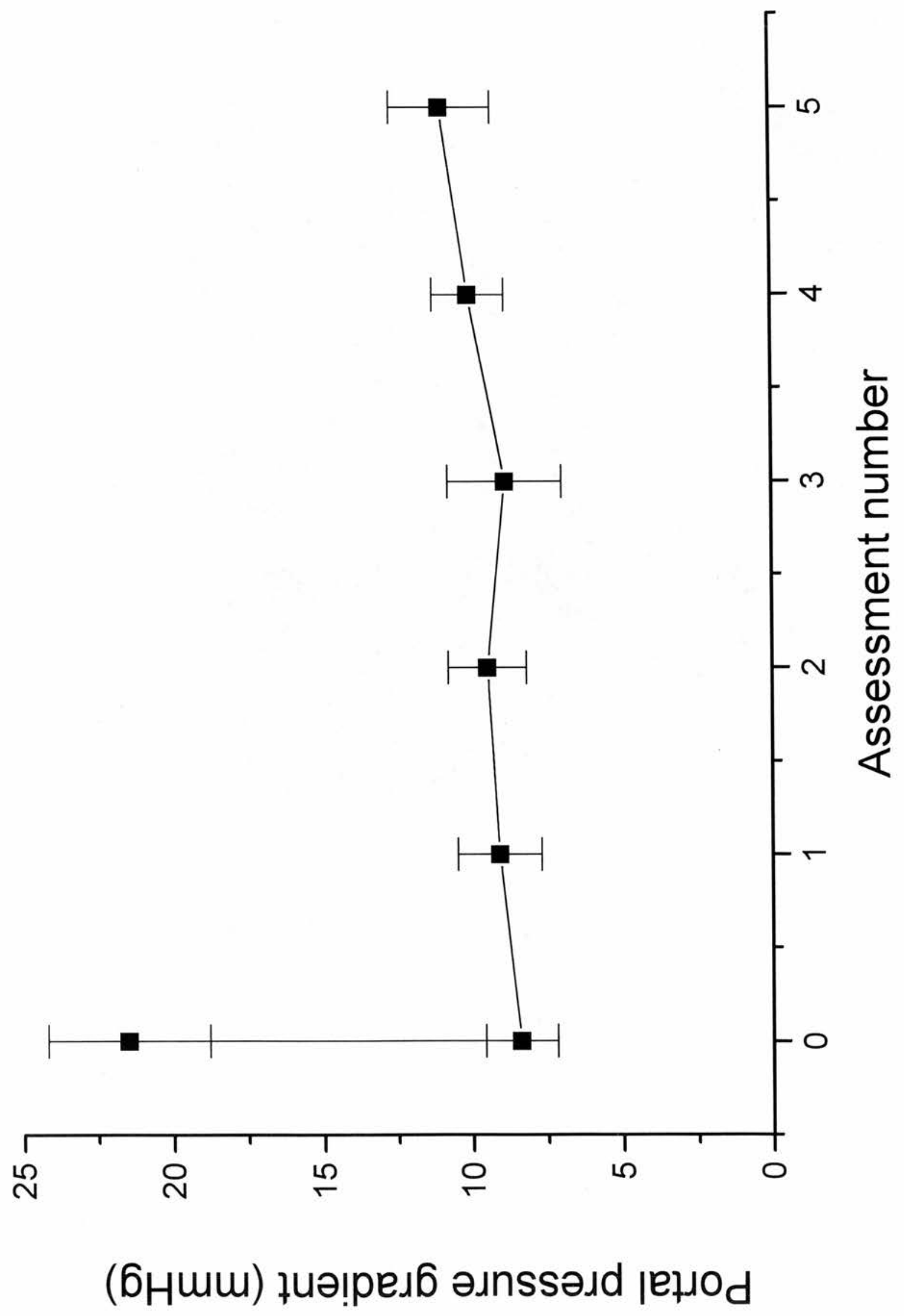




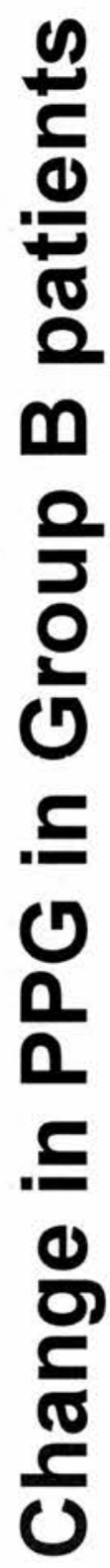

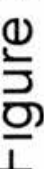

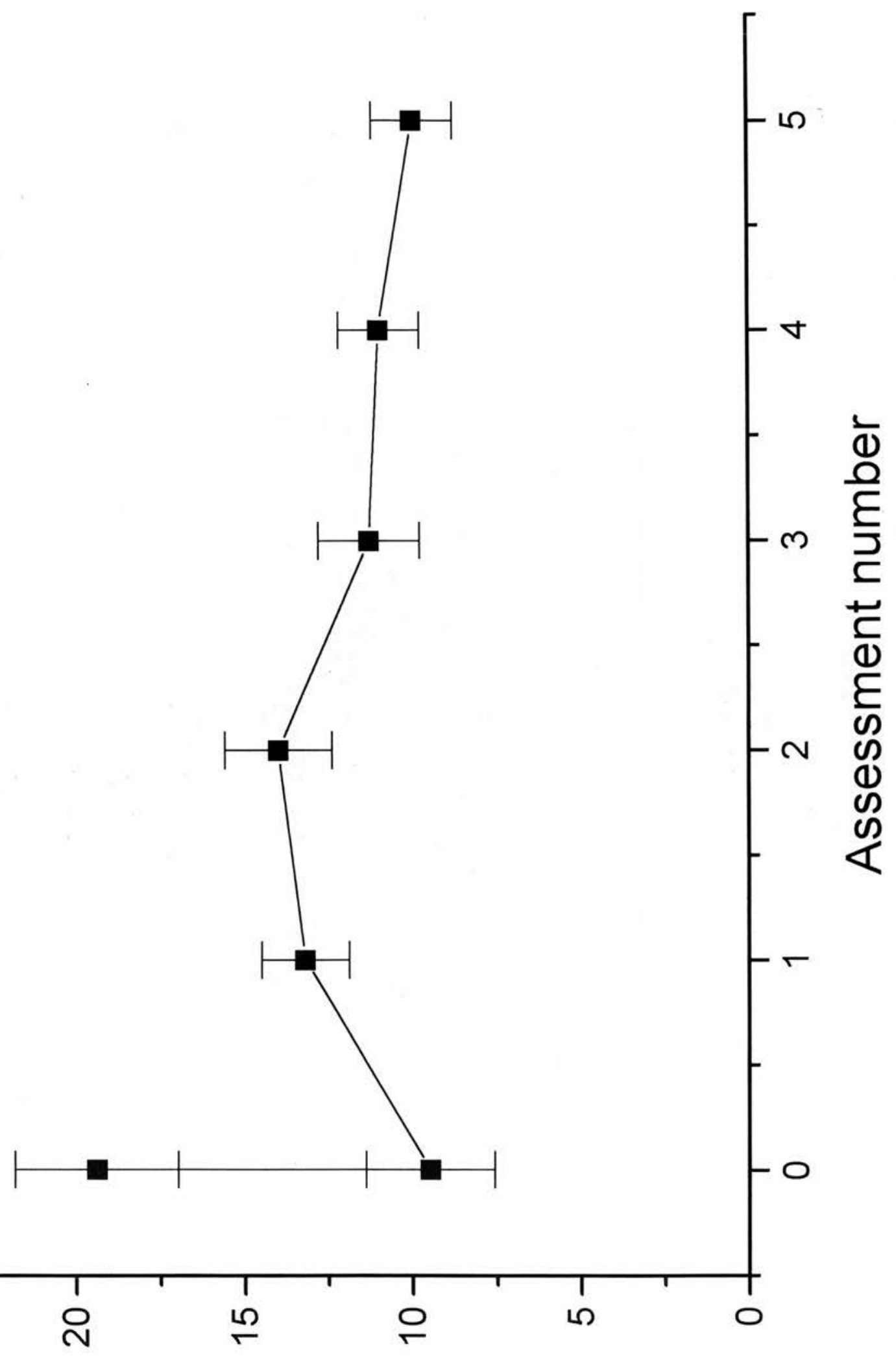

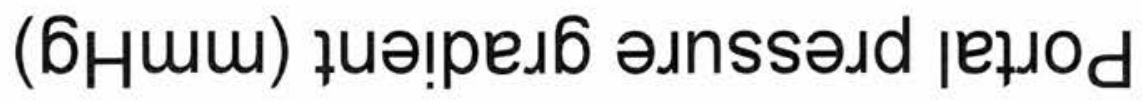




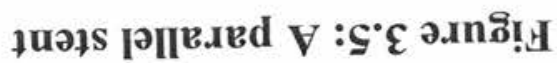

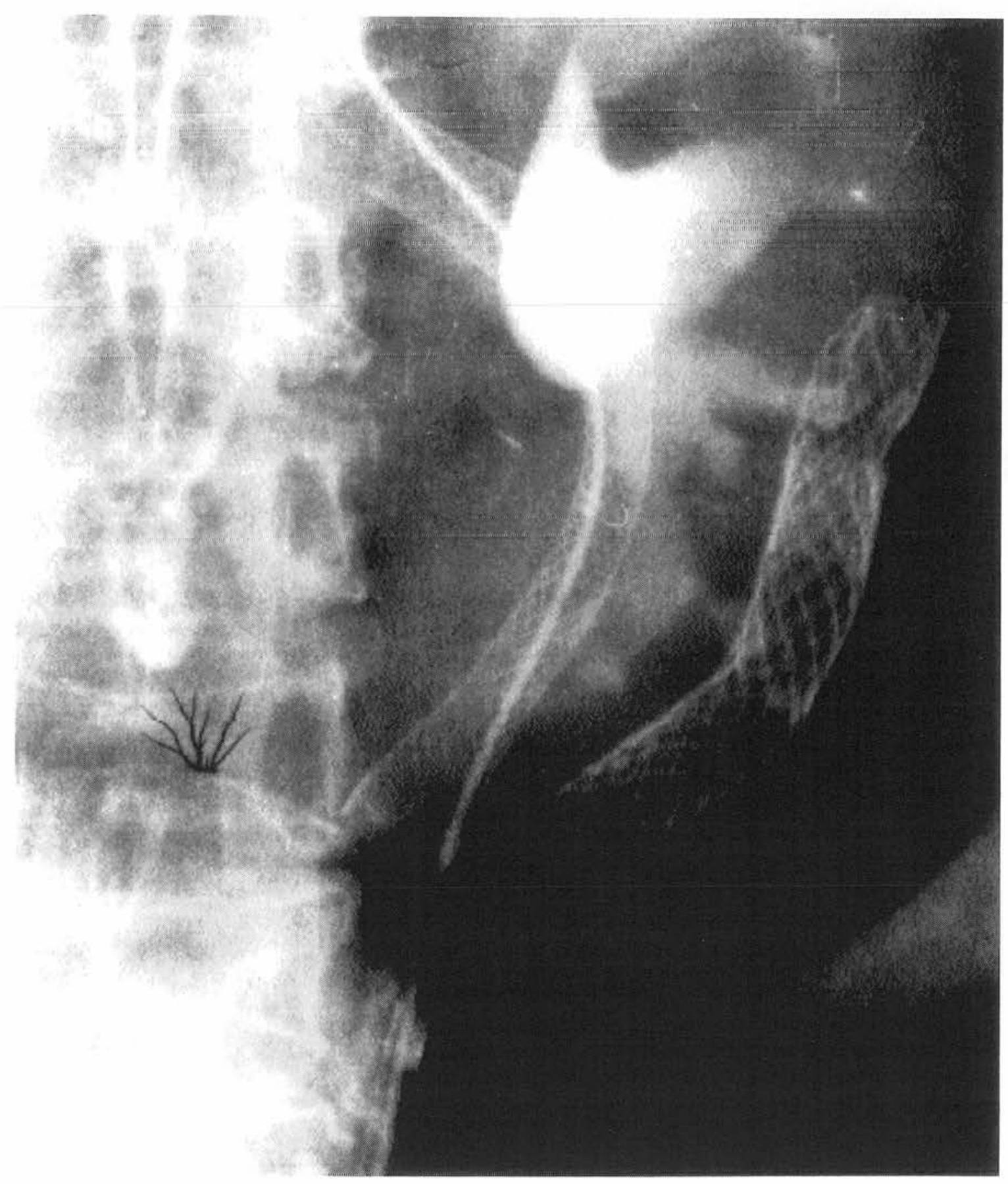




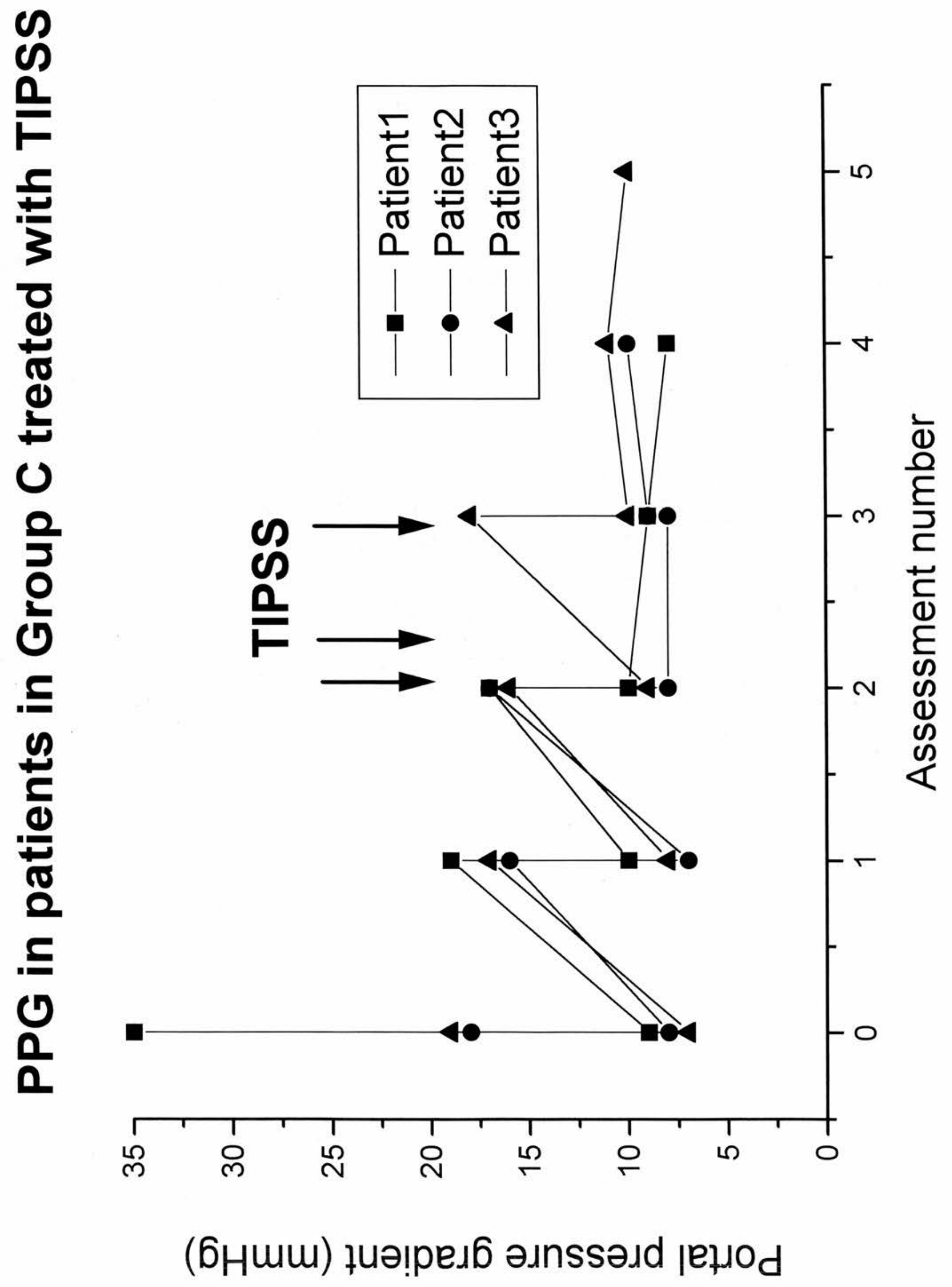




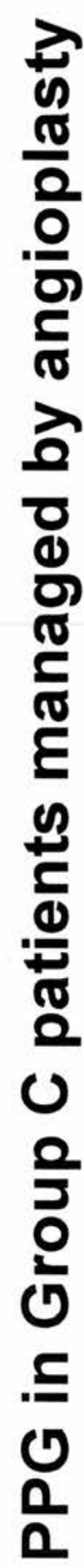

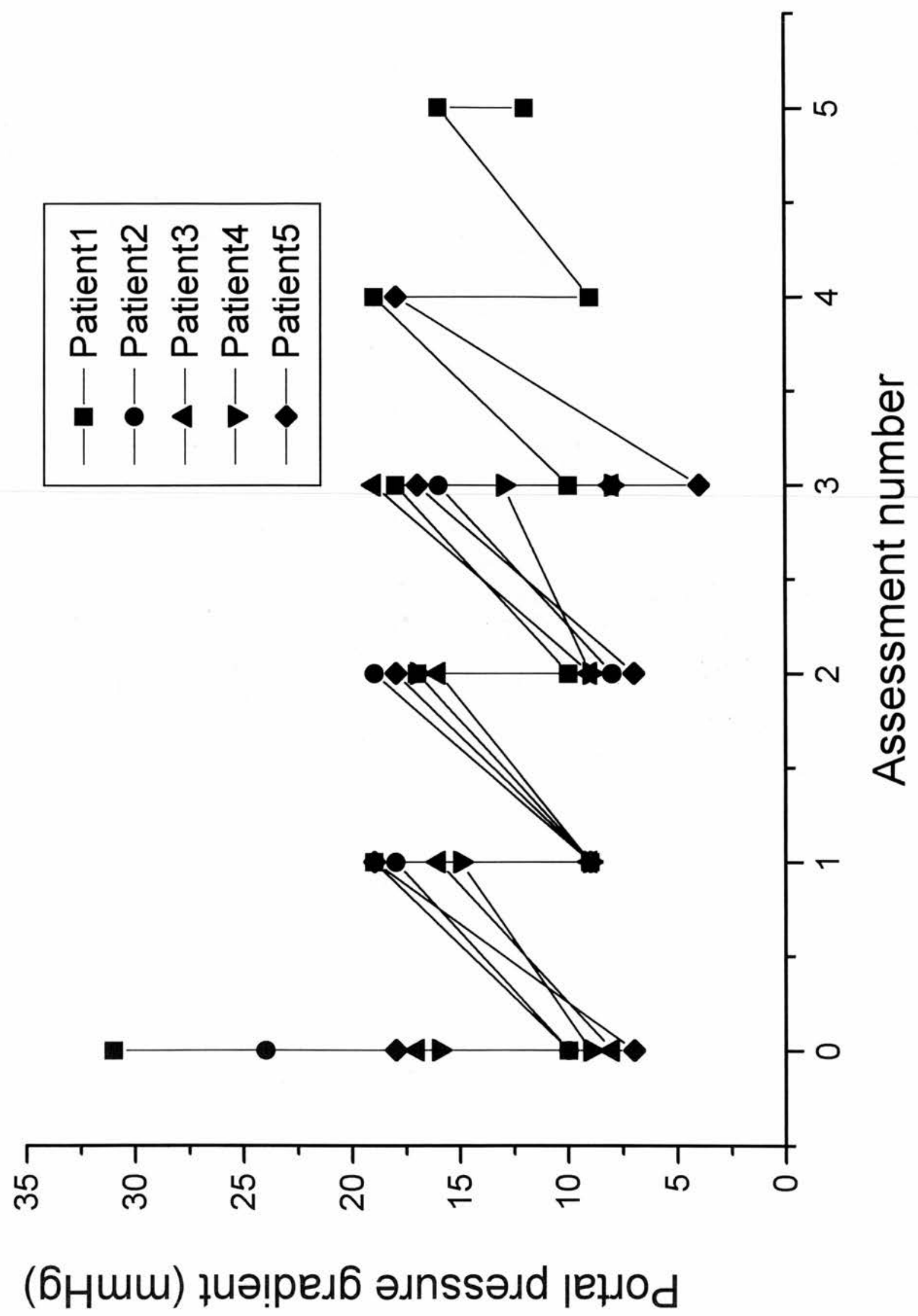




$$
9
$$




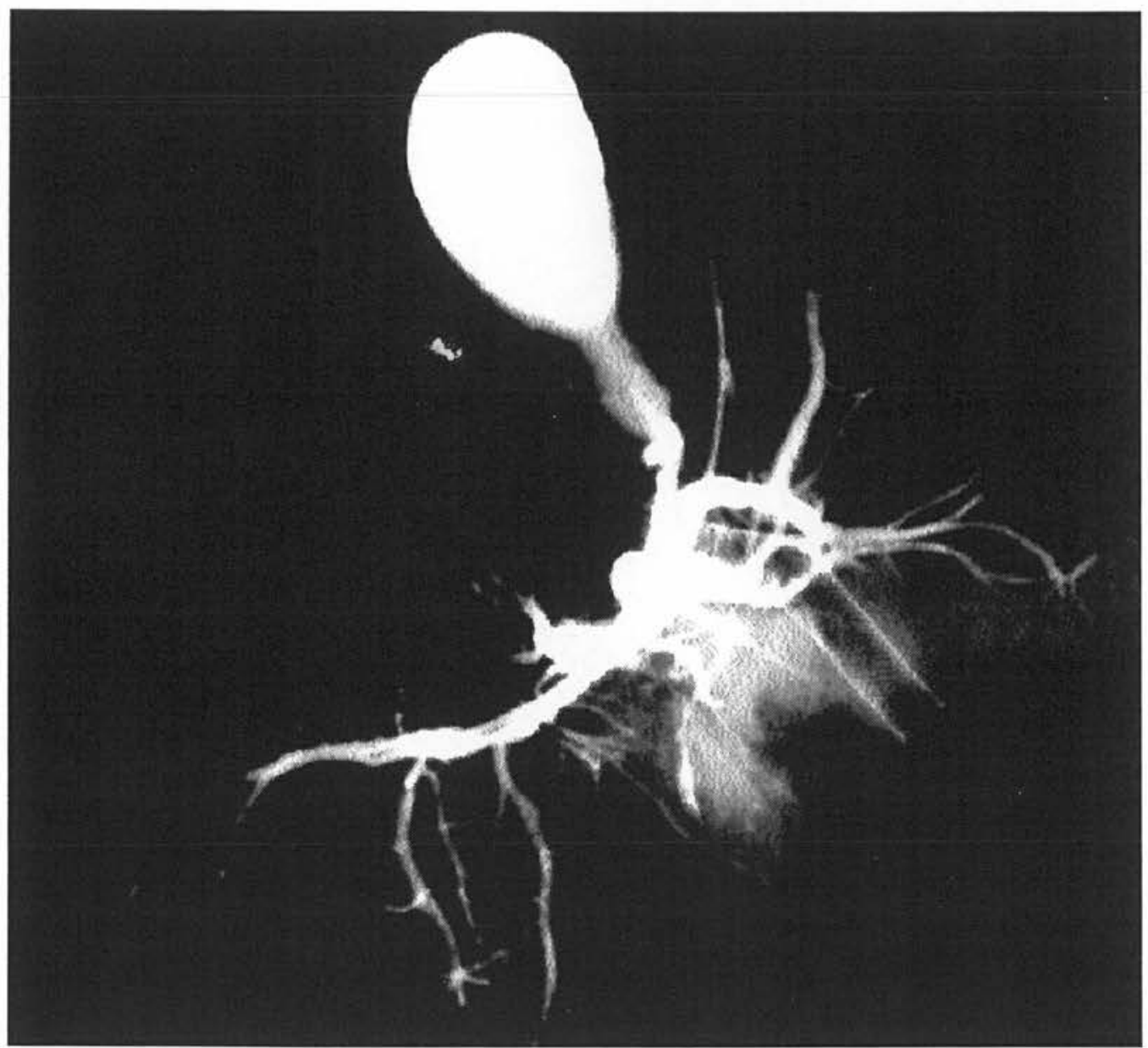

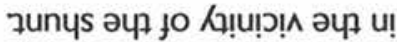

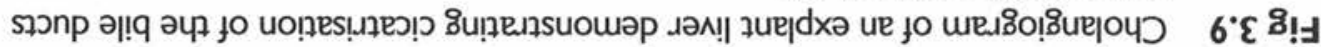


containing fibrous tissue was seen in all 8 patients with bile in the thrombus in 5. In addition, 2 of these patients had biliary epithelium in the biopsy specimens (Figure 3.10).

Explanted livers Four of the 10 explanted livers showed shunt stenosis. Three were severe $(>70 \%)$ and the other was mild $(30 \%)$. No stenosis was seen in the other 6 .

Composition of the lining : The shunt was lined with a white fibrous structure which extended from the point of insertion of the shunt in the hepatic vein to the lower limit of the shunt extending beyond the portal vein bifurcation. Microscopically, it was composed of organising thrombus and fibrous tissue with evidence of endothelialisation (Figure 3.11 and 3.12).

Occluding "thrombus": Macroscopic examination of the contents of the shunt in 3 patients with severe stenosis showed bile stained contents. In all 3 there was a primary thrombus which was adherent to a transected bile duct and was composed of organised thrombus containing bile and occasional biliary epithelium. Associated with the bile there was a granulomatous inflammatory response containing numerous foreign body type giant cells. The underlying bile duct was occlude by thrombus. The propagated thrombus was adherent to the primary thrombus and was histologically similar to the primary thrombus but lacked bile. Exuberant fibrous tissue was responsible for the mild stenosis seen in 1 patient. Bile was not identified in the fibrous tissue, 
nor was there any evidence of transection of a major bile duct. The degree of shunt stenosis was related closely to the size of the duct transected being greater when the size of the duct transected was greater (Figure 3.13, 3.14, $3.15,3.16)$. No evidence of bile was seen either in the pseudo intima or in the shunt in the 5 patients with no stenosis.

In 2 cases there was marked accumulation of stainable iron in the hepatocytes adjacent to the shunt compared with the distant liver tissue and this may be due to local haemorrhage during TIPSS insertion or to shunt related haemolysis. 
Fig 3.10 Photomicrograph of the histopathological appearance of a biopsy specimen obtained from an occluded shunt demonstrating biliary epithelium (positive staining for cytokeratin).

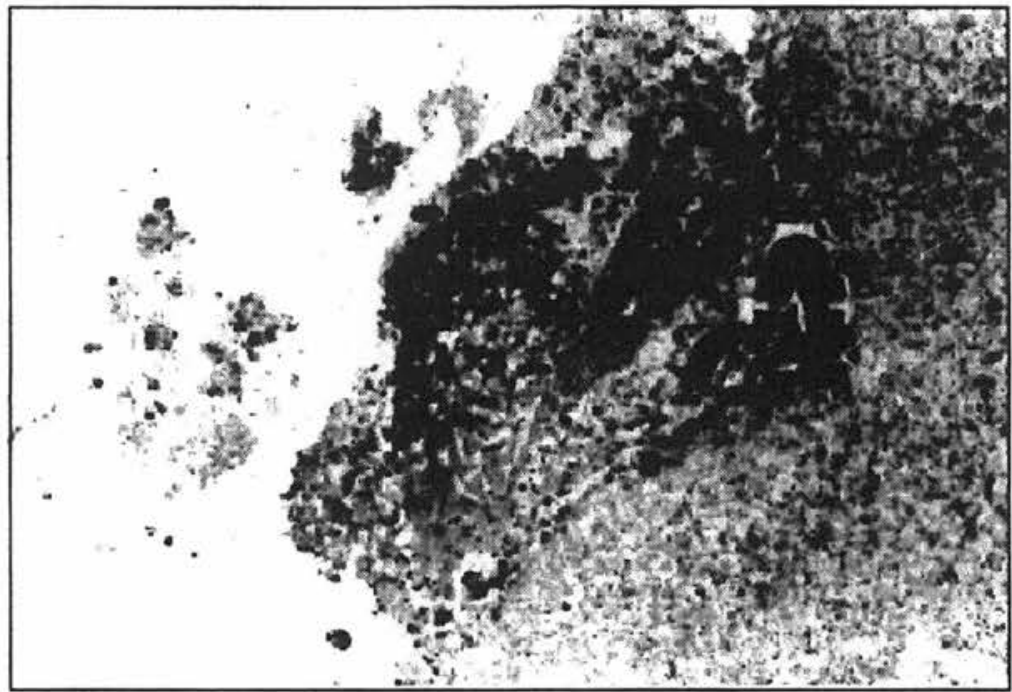

Fig 3.15 and Fig 3.16

Photomicrograph of the histopathological appearance of the thrombus which overlies the transected bile duct demonstrating granulomatous inflammation and bile impregnation.
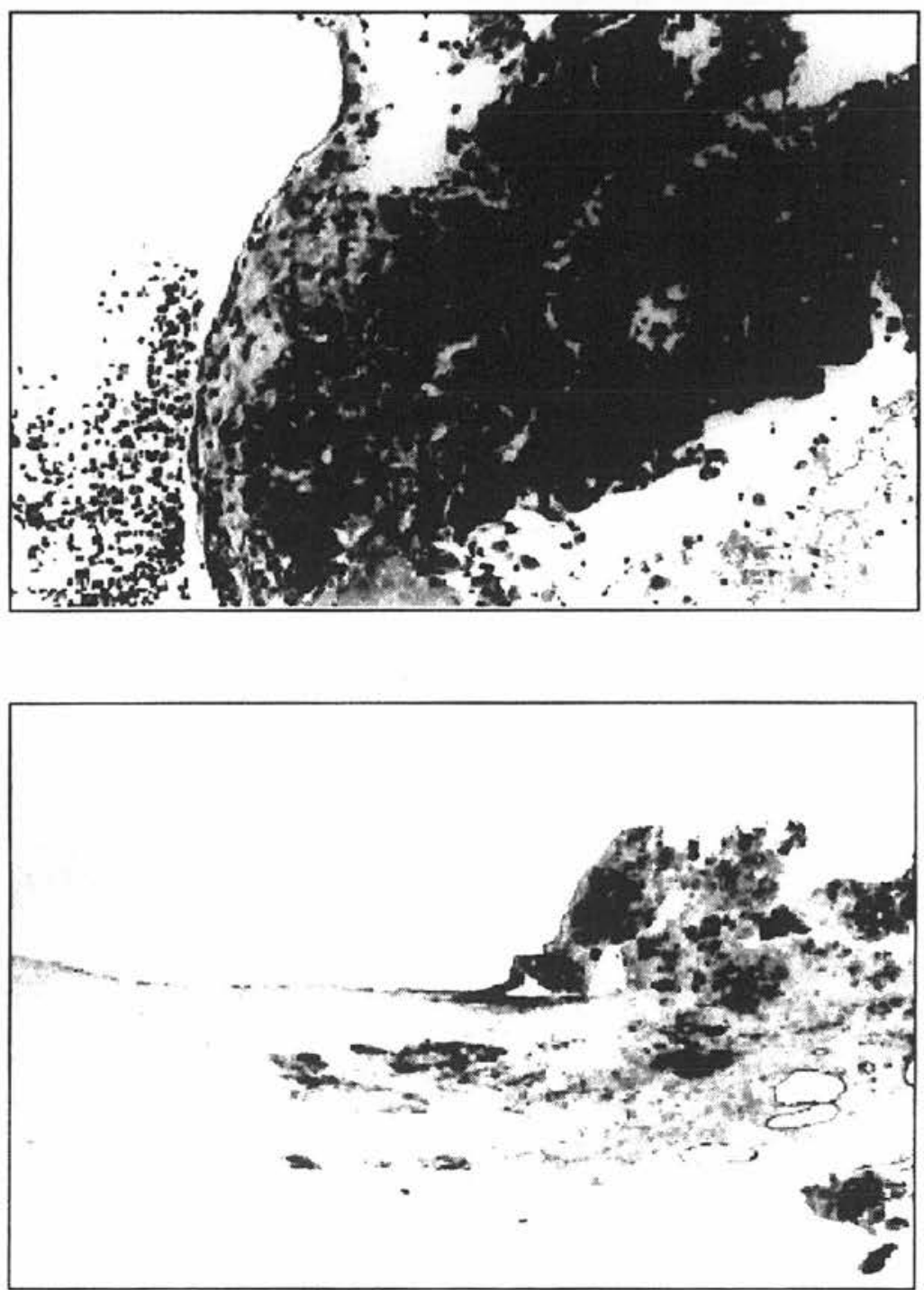
Fig 3.II An endoscope view of the shunt obtained from an explanted liver showing the continuous pseudo-intima.

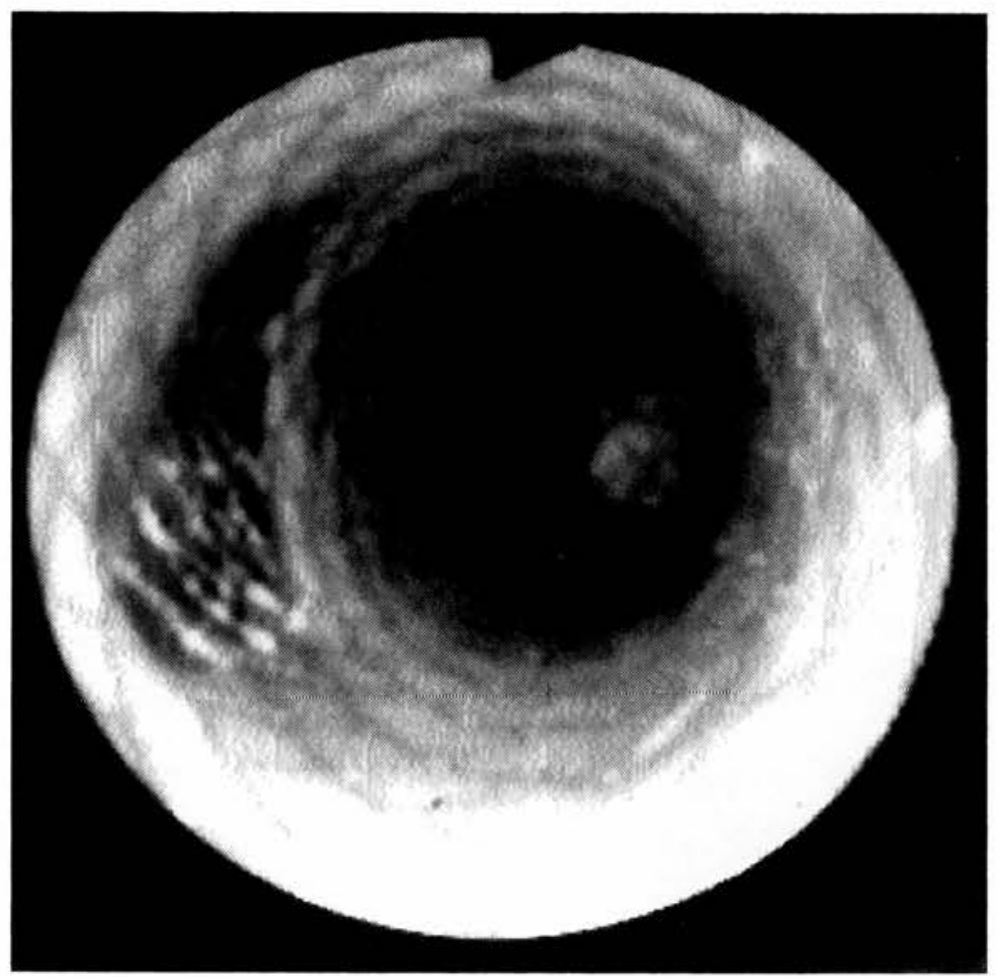

Fig 3.12 Photomicrograph of the lining of the shunt showing the pseudointima.

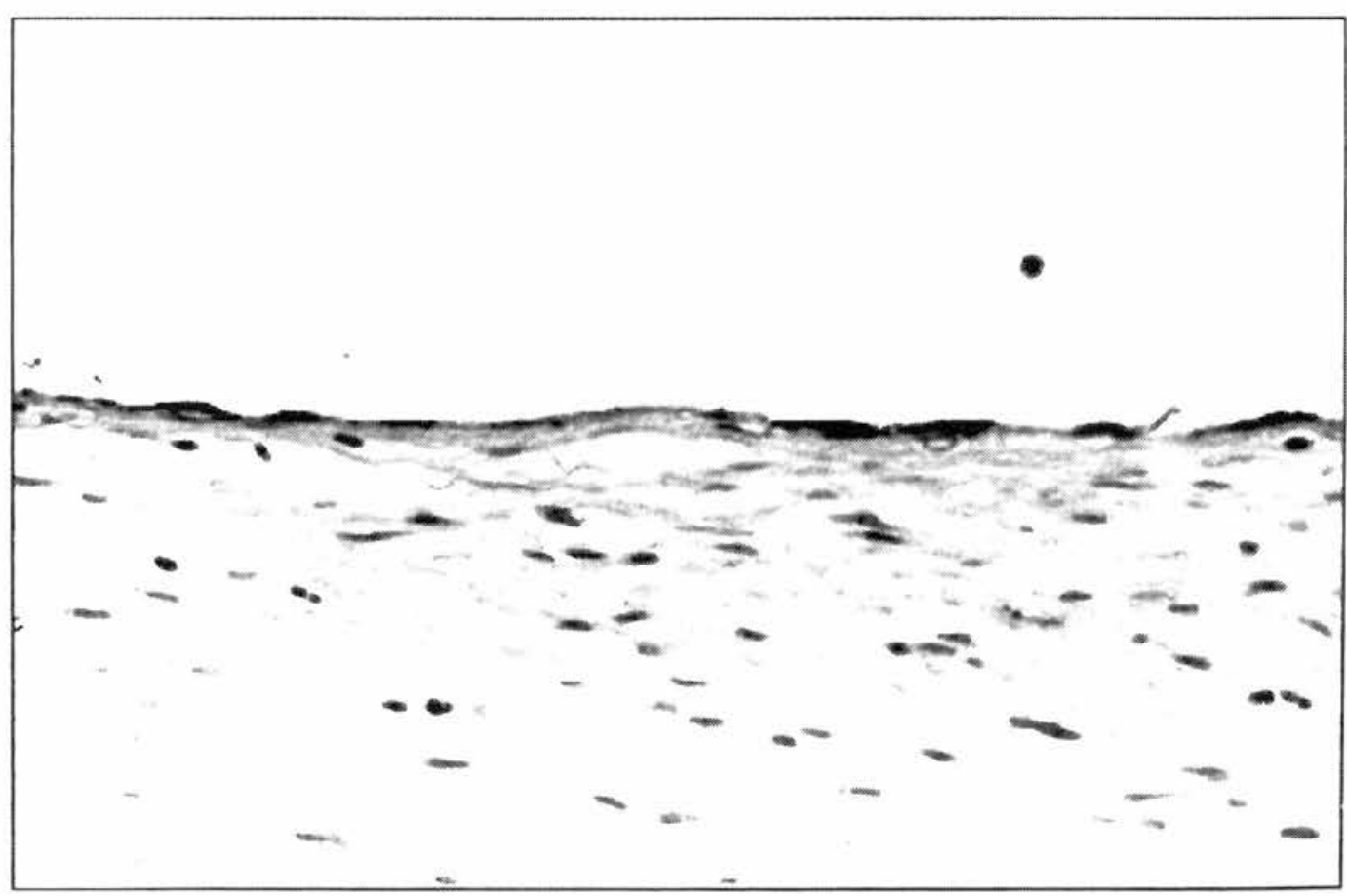


Fig 3.13 Photograph of a shunt that has been dissected out of the liver showing that a major bile duct has been transected.

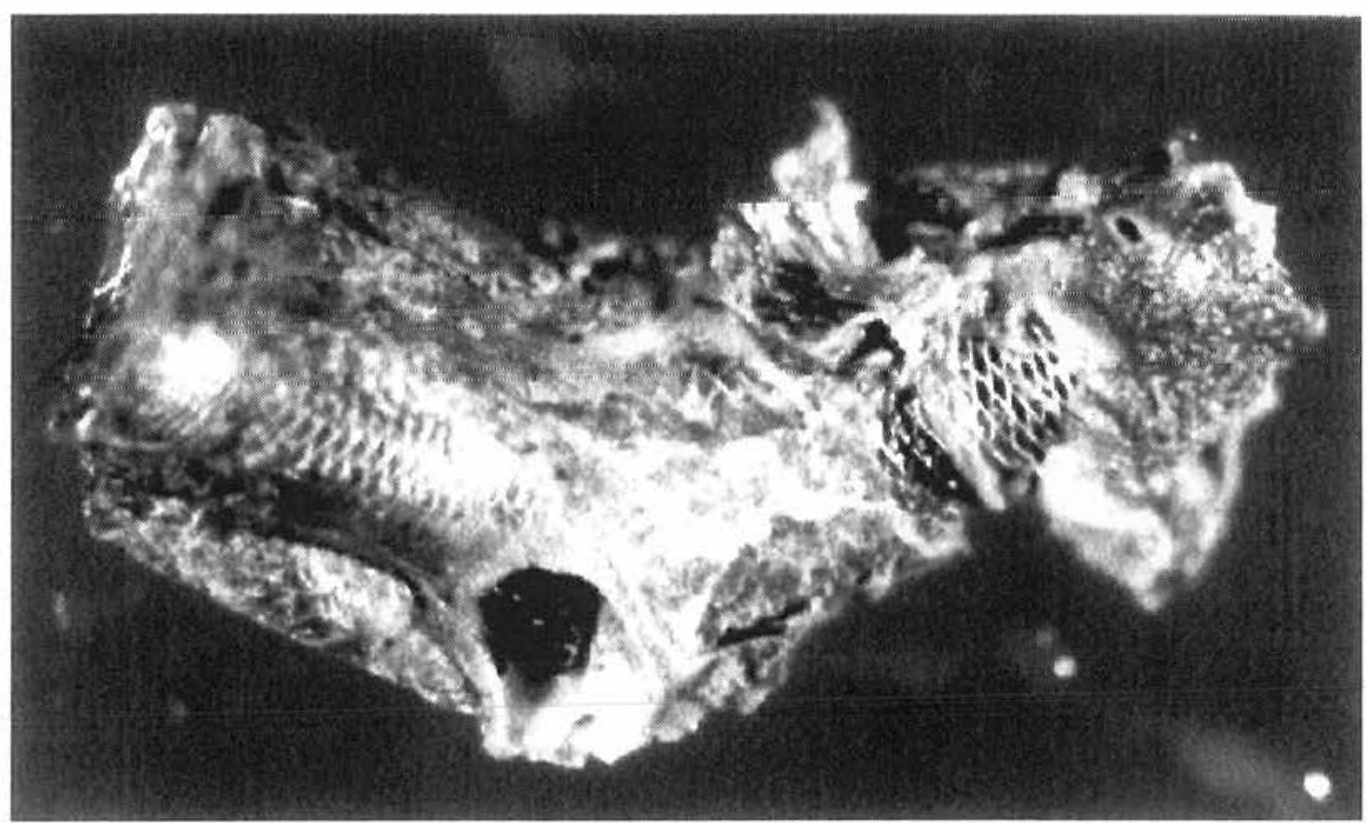

Fig 3.14 Photograph of a shunt which has been dissected out and opened to demonstrate the occluding material.The arrow represents the primary thrombus that overlies the transected bile duct.

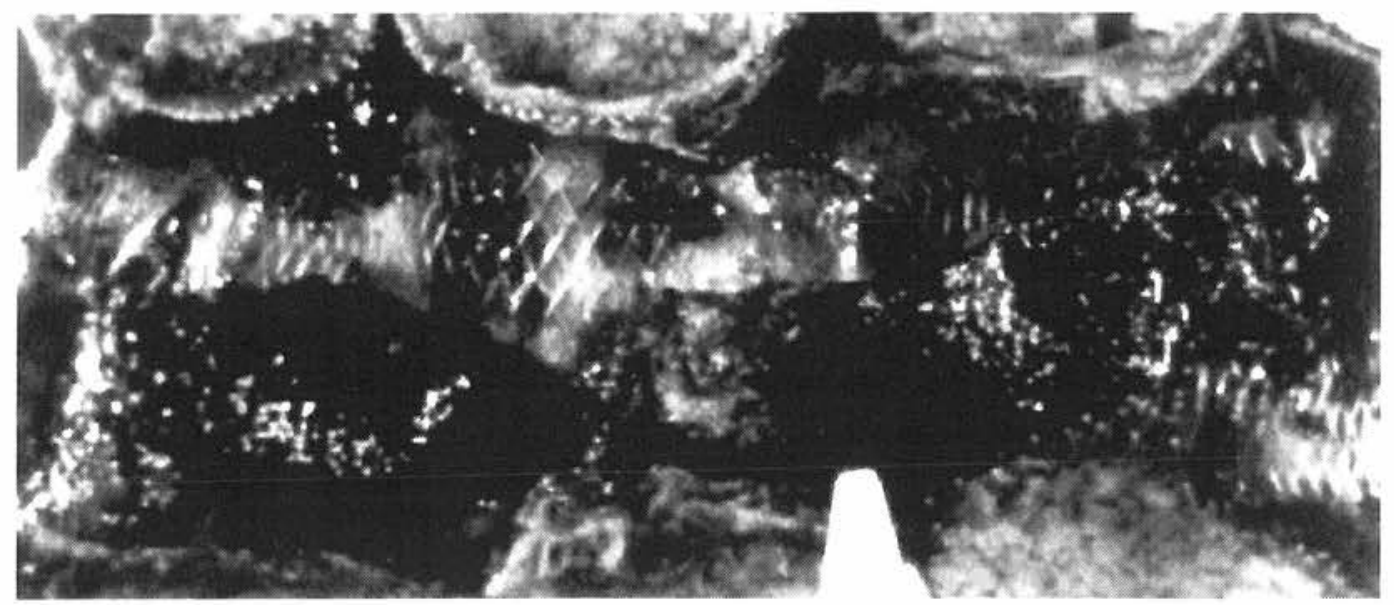




\subsection{SUMMARY}

In conclusion, severe stenosis or occlusion within the TIPSS is most common in the intrahepatic portion, has a primary or bile related and a propagated component and is probably related to the transient formation of biliary venous fistulae caused by introduction of the shunt and made possible by the incomplete interface of the mesh. This may be avoided by altering shunt design such that the parenchymal portion of the shunt may be covered. In view of these findings it may be advisable to establish a new shunt in a patient who has evidence of severe shunt stenosis or shunt occlusion since the persistence of the bile in the shunt will make re-stenosis likely. Alternatively, in the event of a covered stent becoming available, this may be used within the old stent to eliminate the detrimental effect of bile entrapped within the thrombus. Further studies estimating markers of bile leak or thrombogenesis at the time of TIPSS insertion may be able to identify patients likely to develop subsequent shunt dysfunction. 


\section{CHAPTER 4}

Evaluation of TIPSS for variceal haemorrhage in patients with

\section{Cirrhosis}

Uncontrolled Variceal haemorrhage

- Secondary prophylaxis 
4.1 COMPARISON OF TIPSS WITH OESOPHAGEAL TRANSECTION IN THE MANAGEMENT OF UNCONTROLLED VARICEAL HAEMORRHAGE IN CIRRHOSIS

\subsection{INTRODUCTION AND AIMS}

Surgical intervention has been the treatment of choice for patients whose variceal bleeding continues or recurs after 2 sclerotherapy treatments. Oesophageal transection and gastric devascularisation (OT) is the most commonly performed operation for uncontrolled variceal haemorrhage but has high rates of perioperative mortality and late rebleeding (McCormick et.al. 1995).

The aim of this retrospective study was to compare TIPSS with surgery in the treatment of patients with uncontrolled variceal bleeding in a series of patients admitted to a single hospital over a 7 year period. 


\subsection{METHODS}

\subsection{Study Design}

Criteria for entry into the study were defined prior the start of the study. Patients would be included for analysis if they had underlying cirrhosis, uncontrolled variceal haemorrhage, defined as variceal bleeding despite 2 separate sessions of sclerotherapy within 5 days of the first episode of variceal haemorrhage and had been assessed for either OT or TIPSS. Patients would be excluded from the analysis if they exsanguinated before they could be reviewed either for OT or TIPSS. To eliminate any element of bias the data were collected independently by two independent observers.

\subsection{Patient Population}

Two hundred and sixty patients with biopsy proven cirrhosis were referred to the Royal Infirmary of Edinburgh with variceal haemorrhage over a 7 year period (1988-1991 - 124, 1991-1994 - 136). The senior medical and surgical teams who have an interest in variceal bleeding looking after these patients have been constant over this period with the exception of the availability of TIPSS as a therapeutic alternative since July 1991.

Routine management of patients with variceal haemorrhage involved resuscitation followed by urgent intravariceal endoscopic sclerotherapy with $5 \%$ ethanolamine oleate or $2 \%$ sodium tetradecyl sulphate. In the event of continued or recurrent bleeding; balloon tamponade, pharmacological 
therapy and repeat sclerotherapy were used individually or in various combination.

During this period 41 patients $(15.8 \%)$ were diagnosed as having uncontrolled variceal haemorrhage of which $38 \mathrm{form}$ the basis of this study. Similar percentages of patients in both study periods were defined as having uncontrolled variceal haemorrhage (1988-1991 - 15.3\%, 1991-1994-13.1\%). Three patients were excluded because of rapid exsanguinating bleeding before surgical review was possible. Between 1988 and July 1991 all patients with uncontrolled bleeding were treated by transection and since then TIPSS has been used for all the patients with uncontrolled bleeding.

Four other patients had uncontrolled variceal haemorrhage as a result of extrahepatic portal vein obstruction in 3 and non cirrhotic portal fibrosis in 1. These patients were managed successfully by creation of surgical portasystemic shunts.

Of the 38 patients there were 19 each in the TIPSS and the OT groups. The patients in the 2 groups were well matched for age, sex, aetiology of underlying liver disease, its severity and its complications (Tables 4.1). Severity of liver disease was defined at the time of the initial bleed. Five patients with uncontrolled variceal bleeding were in hepatorenal failure, defined as raised plasma creatinine, oliguria and intense sodium retention in spite of a normal central venous pressure. Of the 8 patients with active infection, 1 had fungal septicemia prior to TIPSS. Four patients were diet controlled diabetics (TIPSS 3 , surgery 1 ), 1 had underlying ischemic 
heart disease (surgery) and 1 was receiving propranolol for the treatment of systemic hypertension.

The majority of the patients in both groups were bleeding from oesophageal varices but 11 were bleeding from oesophagogastric varices. None of these patients had isolated gastric varices. Nine of these 11 patients were in the TIPSS group. There was no significant difference in the number of sessions of sclerotherapy, nor the requirement for blood transfusion. A similar number in the 2 groups were haemodynamically unstable (blood pressure of less than $100 \mathrm{mmHg}$ or persistent tachycardia of greater than 100 per minute) at the time of the respective procedures despite greater number of patients in the TIPSS group requiring mechanical ventilation (Table 4.1). The mean duration of time from first bleed to referral was 2.6 days (range 1-6 ) in the TIPSS group and 3.2 days (range $0.25-7$ days) $(p=n s)$ in the surgical group. 
Table 4.1

Patient characteristics

\begin{tabular}{|c|c|c|c|}
\hline Patients ( $n=38$ ) & TIPSS (N=19) & Surgery $(n=19)$ & p-value \\
\hline Age & $57.1( \pm 2.3)$ & $54.9( \pm 4.2)$ & NS \\
\hline $\operatorname{Sex}(M / F)$ & $8 / 11$ & $11 / 8$ & NS \\
\hline \multicolumn{4}{|l|}{ Aetiology } \\
\hline Alcoholic cirrhosis & 15 & 14 & NS \\
\hline Cryptogenic cirrhosis & 4 & 3 & \\
\hline Others & 0 & 2 & \\
\hline \multicolumn{4}{|l|}{ Child class } \\
\hline Class A & 3 & 3 & NS \\
\hline Class B & 3 & 5 & \\
\hline Class C & 13 & 11 & \\
\hline Ascites & 14 & 12 & NS \\
\hline Hepatorenal failure & 3 & 4 & NS \\
\hline Encephalopathy & 3 & 3 & NS \\
\hline Sepsis & 5 & 3 & NS \\
\hline \multicolumn{4}{|l|}{ Bleeding site } \\
\hline Oesophageal varices & 10 & 17 & NS \\
\hline Oesophagogastric varices & 09 & 02 & \\
\hline Sclerotherapy sessions & $2.3( \pm 0.6)$ & $3.1( \pm 0.3)$ & NS \\
\hline Balloon tamponade & 14 & 13 & NS \\
\hline Blood transfusion (units) & $10.2( \pm 1.3)$ & $13.1( \pm 0.9)$ & NS \\
\hline Mechanical ventilation & 05 & 02 & NS \\
\hline Haemodynamic instability & 13 & 11 & NS \\
\hline
\end{tabular}

Results expressed as mean and standard error 


\subsection{TIPSS Procedure}

TIPSS was performed by the standard procedure and has been described in detail in section 3.21 .

\subsection{Oesophageal Transection And Devascularisation}

Oesophageal transection and devascularisation was performed by the well described method using a stapling gun (Burroughs et.al. 1989, McCormick et.al. 1992) by either a consultant surgeon or by a senior registrar under the supervision of a consultant surgeon. Splenectomy was not routinely performed. Gastrografin swallow was performed in all patients following surgery, and a Doppler ultrasonography to ensure adequate shunt function was carried out after TIPSS.

\subsection{Definitions and analysis}

The definitions used were the same as described in section 3.221.

\section{Follow up and Analysis}

Patients in the TIPSS group were assessed clinically, using Doppler ultrasonography and portography at 3 and 6 months and 6 monthly thereafter. Earlier clinical and portographic assessment was made in the patients developing complications. Patients treated with OT were assessed routinely at 3 month intervals and earlier in case of complications. The patients with TIPSS have been followed for 7 patient years (longest 20 months) and those in the OT group for 6 patient years (longest 23 months). 
All results were expressed as mean and standard error of the mean and the difference in the 2 groups tested using unpaired $t$ - test. Survival and rebleeding were analysed on an intention to treat basis using the Kaplan Meier method. 


\subsection{RESULTS}

Overall outcome of the patients in the 2 groups is summarised in Figure 4.1. Control of bleeding was achieved in all patients in the TIPSS group in whom a shunt could be constructed (17 patients). Failure to insert a shunt in 2 patients was due to an inability to puncture the portal vein. The mean portal pressure gradient was reduced from $22.2( \pm 1.2)$ to $9.7( \pm 0.7) \mathrm{mm} \mathrm{Hg}$. Initial control of bleeding was achieved in all patients having successful surgery.

Figure 4.1, The outcome of patients with uncontrolled variceal haemorrhage in the TIPSS and oesophageal transection groups. Numbers within brackets refer to the number of patients.

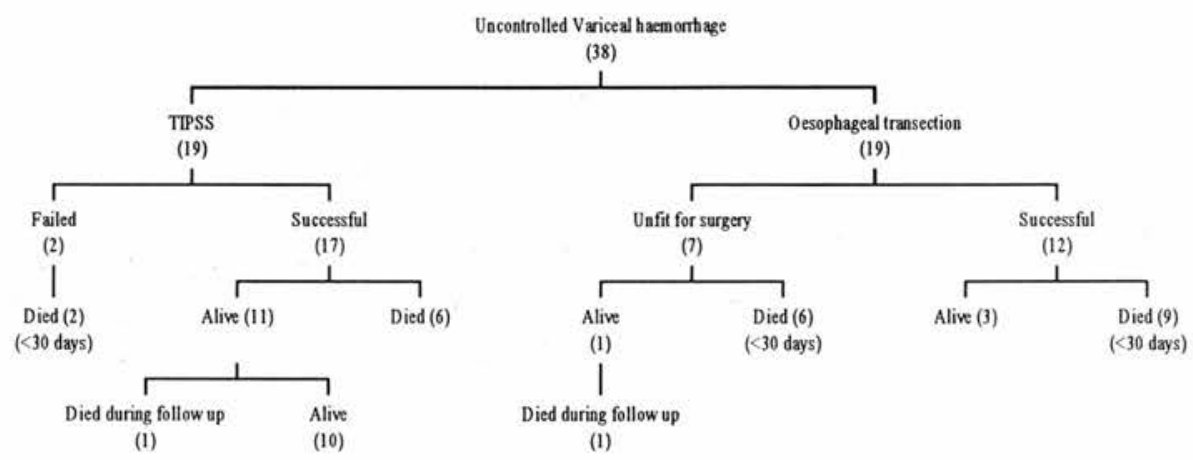

\subsection{Mortality}

Early mortality was significantly higher in the OT group as compared with the TIPSS group (OT $78.9 \%$, TIPSS $42.1 \%)(p<0.05)$ (Figure 4.2$)$. Long term survival in the patients who left the hospital was similar.

Eight of the 9 deaths in patients with TIPSS occurred within 30 days of the procedure. Two patients died after failure to insert a TIPSS, one from massive variceal haemorrhage and the other following subsequent 
oesophageal transection. One death was procedure-related and due to rupture of the extrahepatic part of the portal vein, and 3 patients died from a fulminant liver failure syndrome with characteristic haemodynamic disturbances, hypoglycemia, renal failure, acidosis and intracranial hypertension ( 2 patients). The patient with Candida septicemia prior to TIPSS died from uncontrolled sepsis and 1 patient died from hepatorenal failure. One patient died 6 months after TIPSS from end stage liver disease and 1 underwent orthotopic liver transplantation for intractable encephalopathy.

In the OT group 7 patients were considered too ill for the operation. Six of the 7 patients considered too ill for surgery died within 30 days of the referral, 5 from the effects of uncontrolled bleeding and the sixth from hepatorenal failure. The seventh patient required 2 further sclerotherapy treatments and recovered to leave the hospital but she died 18 months later from end stage liver failure. Nine of the 12 patients undergoing OT died within 30 days of the variceal bleed. Three deaths were related to complications of the procedure (anastomotic leak - 1, cardio respiratory arrest - 2), 3 from sepsis and 3 patients from early rebleeding. One further patient who left the hospital rebled and died 5 months later from end stage liver disease.

\subsection{Rebleeding}

Three of the 19 patients in the TIPSS group rebled from varices $(15.7 \%)$ during the index admission; related to an inadequate shunt in 1 and shunt thrombosis in 2. No further episodes of rebleeding occurred in the TIPSS 


\section{Kaplan Meier Analysis For Survival}

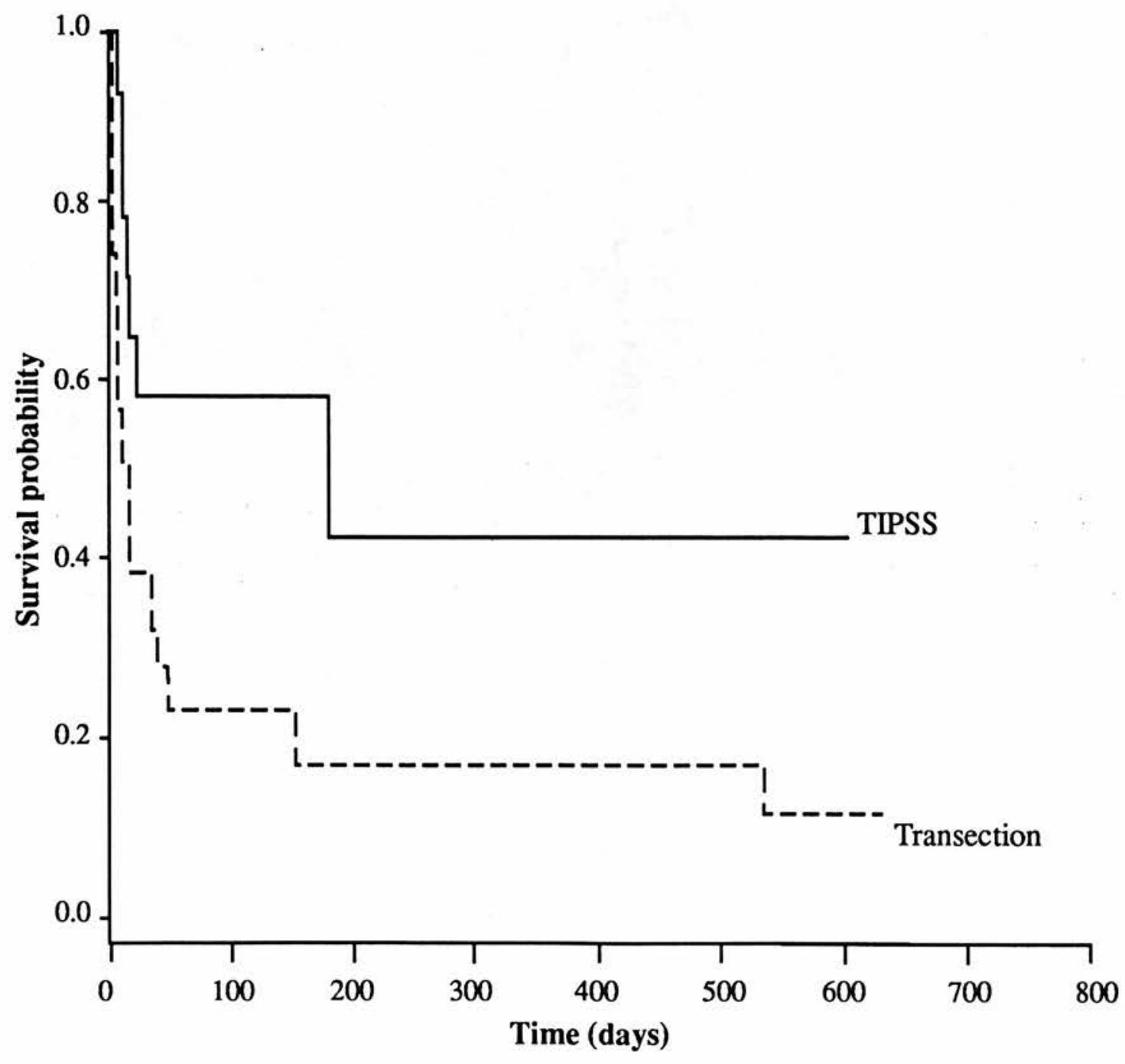

Figure 4.2 
group during follow up. These were managed successfully by shunt extension and balloon dilatation respectively. One patient bled from sclerotherapy ulcers and responded to treatment with sucralfate. Following OT 5 of the 12 patients rebled $(41.6 \%)$ from varices; 3 during the same hospital admission and 2 during follow up. The outcome of variceal rebleeding in the OT group was much worse with 4 of the patients dying from the effects of the bleed. Bleeding was controlled in the fifth patient following an emergency TIPSS. Overall, there was no statistically significant difference in the rates of rebleeding.

\subsection{Encephalopathy}

Two patients with TIPSS developed encephalopathy that was absent prior to the procedure. One patient required reduction in shunt size and was subsequently transplanted. The other was managed satisfactorily with protein restriction and lactulose. In the OT group 1 patient developed encephalopathy which was successfully treated conservatively. Overall, clinically detectable encephalopathy was evident in 22 and $25 \%$ of patients in the OT and TIPSS groups respectively.

\subsection{OTHERS}

\section{Ascites And Hepatorenal Failure}

Ascites improved in all patients with TIPSS. All patients with hepatorenal failure died in both groups, although transient improvement in renal function was noted in 1 patient with TIPSS. Reaccumulation of ascites occurred in 3 
patients with TIPSS (spontaneous bacterial peritonitis 2, hepatic vein stenosis 1) which were managed successfully conservatively.

\section{$\underline{\text { Sepsis }}$}

Following TIPSS 3 patients developed infection (staphylococcal septicemia 2 and chest infection 1) which responded readily to antibiotics. One patient with prior Candida associated septicemia died following TIPSS. In the surgical group 6 patients developed sepsis which was fatal in 2 .

\section{Shunt Function}

Shunt insufficiency (defined as an increase in the portal pressure gradient to $>12 \mathrm{mmHg}$ or a rise in the gradient to $>\mathbf{2 0 \%}$ over the base line value) occurred in 5 patients. Three patients rebled from varices and 1 presented with reaccumulation of ascites. One patient was diagnosed to have shunt insufficiency at routine screening. All episodes of shunt insufficiency were successfully treated by balloon angioplasty, extension of the shunt or insertion of a new shunt. Overall, there was no significant change in the mean portal pressure gradient with it increasing to 10.4 (se 1.1) $\mathrm{mmHg}$ at 3 months to 11.2 (se 1.3) at 6 months, 10.9 (se 1.5) to 11.1 (se 2.1) $\mathrm{mmHg}$ at 12 months. 


\subsection{SUMMARY}

The results of this study indicate that TIPSS can be performed successfully in very ill patients who are not fit to undergo OT and is associated with significantly lower rates of early mortality. In this group of very ill patients, it prolongs life and we believe, should be regarded as the preferred mode of treatment as compared with OT for uncontrolled variceal haemorrhage. A randomised prospective study comparing these treatments is needed to answer this question definitively. 


\subsection{TIPSS VS VBL IN THE SECONDARY PROPHYLAXIS OF VARICEAL \\ BLEEDING IN PATIENTS WITH CIRRHOSIS. A RANDOMISED AND CONTROLLED STUDY}

\subsection{INTRODUCTION AND AIMS}

Acute variceal haemorrhage can be controlled in about $90 \%$ patients using endoscopic approaches such as variceal band ligation (VBL) or sclerotherapy, pharmacological agents, or balloon tamponade. When compared with placebo endoscopic variceal sclerotherapy has been shown to reduce the rates of variceal rebleeding and mortality (Westaby et.al. 1985). There are now 7 fully published trials in the literature comparing sclerotherapy with VBL which were combined in a meta-analysis (Laine et.al. 1993). This included 547 patients and concluded that VBL carried a significantly lower rate of rebleeding, mortality, complications such as oesophageal stricture, and the number of treatments required for variceal eradication. VBL is now considered by most as the endoscopic treatment of choice for patients with oesophageal variceal bleeding (Laine et.al. 1995).

TIPSS reduces the portal pressure thereby controlling variceal haemorrhage. It has been shown in numerous uncontrolled studies to reduce rebleeding in patients with recurrent variceal hemorrhage and is now considered the preferred modality of treatment for uncontrolled variceal hemorrhage (LaBerge et.al. 1993, Rossle et.al. 1994, Jalan et.al. 1994). However, there have been some concerns in the literature about 
deterioration in liver function and an increase in the incidence of chronic hepatic encephalopathy after TIPSS (LaBerge et.al. 1993, Rossle et.al. 1994, Jalan et.al. 1994).

The aim of this randomised and controlled study was to compare TIPSS with VBL in the secondary prophylaxis of variceal haemorrhage in patients with cirrhosis referred to a single centre with the first episode of variceal haemorrhage. 


\subsection{METHODS}

\subsection{Patient Selection}

Between May 1993 and December 1995 a total of 105 patients were referred with endoscopically proven variceal haemorrhage. Of these, 58 patients were enrolled into the trial. Patients were included if they were between 18 and 75 years of age, had underlying cirrhosis, and presented with the first episode of oesophageal variceal haemorrhage. Patients were excluded from the study for the following reasons; less than 18 years of age $(n=2)$, patients who rebled from varices within 24 hours of the initial endoscopy $(n=12)$, bleeding from ectopic varices such as gastric, duodenal or rectal varices $(n=14)$, previous endoscopic treatment for variceal bleeding $(n=18)$, hepatorenal failure $(n=3)$, hepatic or extra-hepatic malignancy $(n=2)$, thrombosed portal vein (Colour flow Doppler ultrasonography) $(n=3)$, or failed to give informed consent $(n=3)$.

\subsection{Informed Consent, Ethical Approval, Randomisation and Study Design}

The study design was approved by the Lothian Regional Ethics Committee and informed consent was obtained from all the patients or their next of kin. Patients with active bleeding were resuscitated and underwent an upper gastrointestinal endoscopy within 6 hours of admission. If the patients were bleeding actively from varices, endoscopic variceal sclerotherapy was performed using $5 \%$ ethanolamine oleate, otherwise they underwent VBL. 
Treatment assignment was achieved using the closed envelope method in batches of 25 patients. Patients were randomised 24 hours after their first endoscopic treatment if no further haemorrhage occurred. Randomisation was performed if patients fulfilled the criteria for inclusion into the study, had no exclusion criteria and had endoscopically confirmed variceal haemorrhage which was defined as bleeding from an oesophageal varix at the time of endoscopy or the presence of large oesophageal varices with blood in the stomach and no other recognisable cause of bleeding. The assigned treatment (VBL or TIPSS) was performed as soon as possible after randomisation.

\subsection{Transjugular Intrahepatic Portosystemic Stent-Shunt}

Details of the TIPSS procedure are described in section 3.21.

\subsection{Variceal Band Ligation}

All the endoscopic procedures and VBL were performed or closely supervised by one of the three experienced endoscopists as described by Steigmann et.al (1992). Each of the varices were ligated with a single band using an end-viewing endoscope and an outer adapter (Bard Intervention Products, Tewkesbury, Massachusetts, USA). The ligation was started as near the oesophago-gastric junction as possible and extended in a spiral upwards to $4-5 \mathrm{~cm}$ above the oesophago-gastric junction. At each session each variceal cord was ligated using a single band. Eradication was 
confirmed when the proposed column had either disappeared or could not be 'sucked' into the ligator.

\subsection{Follow Up Treatment}

Care of the patients in both the groups was identical and involved complete clinical and biochemical assessment at 1 and 3 months, and 6 monthly thereafter. The presence or absence of encephalopathy was assessed using Parson-Smith criteria (Parson Smith et.al. 1957).

TIPSS group: Shunt function was assessed using Doppler ultrasonography 1 week after insertion of the shunt. Routine portography was performed at 1 month after TIPSS and then at 6 monthly intervals.

VBL group: Repeat endoscopy and band ligation was performed at weekly intervals until variceal eradication, and then at 3 and 6 months and 6 monthly thereafter.

\subsection{Definitions and Management of Complications}

All the definitions used were from a consensus meeting (De Franchis et.al. 1992)

Rebleeding: This was defined as subsequent upper gastrointestinal bleeding manifested by hematemesis and/or malena, and a reduction in haemoglobin by $20 \mathrm{~g} / \mathrm{l}$ and requiring an unscheduled endoscopy. It was classified as originating from oesophageal varices if it fulfilled the criteria outlined above. Alternatively, the site of rebleeding was noted. 
Patients with variceal rebleeding were resuscitated and managed as follows. TIPSS group - Banding/Sclerotherapy at endoscopy followed by urgent portography and measurement of PPG. Shunt complications were dealt with as explained below.

VBL group - Banding/Sclerotherapy at endoscopy followed by continuation on the banding programme.

Uncontrolled variceal bleeding: This was defined as continued bleeding despite 2 separate sessions of endoscopic treatment within five days of the first treatment.

In both groups the patients were resuscitated and the bleeding was temporarily controlled using a Sengstaken tube.

TIPSS group - Urgent portography and treatment of shunt dysfunction.

VBL group - An urgent TIPSS procedure would be performed if the patients had uncontrolled variceal haemorrhage as defined above or at the time of the rebleed they were thought to be bleeding from isolated gastric varices.

Early mortality. Death within 6 weeks of the initial episode of bleeding.

Hepatic encephalopathy: This was defined as detection of encephalopathy, or deterioration in the grade of encephalopathy using the Parsons-Smith criteria (Parson-Smith et.al. 1957). 
TIPSS group - Hepatic encephalopathy was treated with protein restriction and lactulose and reduction in the size of the shunt (Angiomed) (Hauenstein et.al. 1995) if this failed.

VBL group - Hepatic encephalopathy was treated with protein restriction to 40 grams per day and lactulose to ensure at least 2 soft bowel motions per day.

Shunt insufficiency: This was defined as an increase in the PPG to greater than $12 \mathrm{~mm} \mathrm{Hg}$ or an increase in the PPG by $20 \%$ over the post TIPSS value.

Management: Shunt insufficiency was treated with balloon angioplasty, shunt extension or insertion of a parallel stent.

\subsection{Estimation Of The Cost Of The Procedures}

The cumulative duration of time spent by each patient during the period of follow up was estimated. This was subdivided into the amount of time that each patient required in the intensive care unit, in the high dependency unit and on the ordinary wards. Patients were admitted to the intensive care unit if they required mechanical ventilation, to the high dependency unit if they required intensive cardiovascular monitoring and were managed on the ordinary wards if they required hospital treatment. The costs of treatment in the different groups were estimated as they were costed for the National Health Service in the UK. These do not include cost for personnel. Blood 
products are available free at the point of use within the National Health Service.

Cost of TIPSS related procedures

The costs for the TIPSS procedure were divided into consumable cost for TIPSS (£2239), consumable cost for routine portography (£228), consumable cost for angioplasty (£405), consumable cost for shunt extension (£1194), consumable cost for parallel shunt (£2245) and consumable cost for reducing stent $(£ 1000)$.

Cost of variceal band ligation

The standing charge for variceal band ligation and endoscopy is $£ 150$.

Cost of in patient and out-patient care was taken from the Scottish Office Registry for health expenditure and normalised for the date they were treated.

4.228. End Points, Determination Of Sample Size, Study Termination And Statistical Analysis

The primary end point in this study was variceal rebleeding, and the secondary end points were death and complications such as encephalopathy, sepsis, shunt dysfunction in the TIPSS group and oesophageal complications in the VBL group during follow up. Other secondary end point were estimation of the amount of time patients spent in 
the hospital, total number of procedures they underwent and an estimation of the cost in the two groups.

Assuming a reduction in the rate of rebleeding of $25 \%$ in studying patients with the first episode of variceal bleeding (predicted rebleeding; VBL - 35\%, TIPSS - 10\%) (Laine et.al. 1995, Jalan et.al. 1994), the estimated sample size to detect a difference with $\alpha$ and $\beta$ values of 0.05 and 0.20 , would be 27 patients in each group using a one tailed test. Patient recruitment was stopped when the above number was reached and the study was terminated 3 months after the last patient had been recruited. An interim analysis was built into the protocol which was performed by an independent statistician to ascertain whether there was any adverse effects of the new treatment and, if necessary, recalculate the sample size.

All the data were expressed as mean and standard deviation. All the results were analysed on an intention to treat basis. Mortality, rebleeding and shunt insufficiency were analysed using the Kaplan-Meier method. Difference between groups was tested using the Chi square test for qualitative data, unpaired t-test for quantitative data and the log-rank test for the survival and rebleeding data. 


\subsection{RESULTS}

\subsection{Patients}

Of the 58 patients who were enrolled into the study, 27 were randomised to VBL and 31 to TIPSS. Mean follow up in the TIPSS group was $15.7( \pm 10.2)$ months and in the VBL group was $16.8( \pm 10.9)$ months. Patient characteristics and duration of follow up in both the groups were similar (Table 4.2). At initial endoscopy 4 patients in the TIPSS group and 3 in the VBL group were bleeding actively from oesophageal varices. Bleeding in these patients was controlled using endoscopic variceal sclerotherapy with $5 \%$ ethanolamine oleate. The rest of the patients in both groups underwent VBL. None of the patients in either group showed any evidence of continued bleeding at the time of randomisation. Mean time from the first evidence of bleeding to initial endoscopy was $8.5( \pm 0.4) \mathrm{hr}$ in patients in the TIPSS group and $7.4( \pm 0.5) \mathrm{hr}$ in patients in the VBL group. Mean time from the initial endoscopy to randomisation was $1.2( \pm 0.3)$ days in the TIPSS group and 1.4 $( \pm 0.5)$ in the VBL group. 
Table 4.2

Patient characteristics

\begin{tabular}{|c|c|c|}
\hline CHARACTERISTICS & TIPSS $(n=31)$ & VBL $(n=27)$ \\
\hline Age & $55.17( \pm 9.5)$ & $59.9( \pm 8.6)$ \\
\hline $\operatorname{Sex}(M / F)$ & $21 / 10$ & $16 / 11$ \\
\hline \multicolumn{3}{|l|}{ Child Class } \\
\hline $\begin{array}{r}\text { Class A } \\
\text { Class B } \\
\text { Class C } \\
\text { Pugh Score }\end{array}$ & $\begin{array}{l}2 \\
14 \\
15 \\
9.2( \pm 2.3)\end{array}$ & $\begin{array}{l}5 \\
9 \\
13 \\
9.1( \pm 2.6)\end{array}$ \\
\hline \multicolumn{3}{|l|}{ Diagnosis } \\
\hline $\begin{array}{l}\text { ALD } \\
\text { PBC } \\
\text { HBV/HCV } \\
\text { Cryptogenic cirrhosis } \\
\text { CAH } \\
\text { Ascites }\end{array}$ & $\begin{array}{l}26 \\
2 \\
1 / 1 \\
1 \\
- \\
28(90.2 \%)\end{array}$ & $\begin{array}{l}21 \\
3 \\
1 \\
1 \\
1 \\
21(77.7 \%)\end{array}$ \\
\hline Encephalopathy & $7(25.8 \%)$ & $9(33.3 \%)$ \\
\hline Haemodynamic instability & $3(9.7 \%)$ & $2(7.4 \%)$ \\
\hline Mechanical Ventilation & $1(3.2 \%)$ & $2(7.4 \%)$ \\
\hline Blood Transfusion at randomisation (Units) & $5.8( \pm 1.2)$ & $6.3( \pm 3.7)$ \\
\hline \multicolumn{3}{|l|}{ Portal Pressure Gradient (mmHg) } \\
\hline $\begin{array}{l}\text { Pre TIPSS } \\
\text { Post TIPSS }\end{array}$ & $\begin{array}{l}19.4( \pm 1.1) \\
8.9( \pm 1.1)\end{array}$ & - \\
\hline Continued alcohol abuse & $72 \%$ & $76 \%$ \\
\hline Duration of Follow up (months) & $15.7( \pm 10.2)$ & $16.8( \pm 10.9)$ \\
\hline
\end{tabular}

Results expressed as mean and standard deviation. ALD - Alcoholic Liver Disease

$\mathrm{CAH}$ - Chronic active hepatitis, PBC - primary biliary cirrhosis, HBV Hepatitis B virus,

$\mathrm{HCV}$ - Hepatitis $\mathrm{C}$ virus ${ }^{*} A s s e s s e d 1$ week after discharge in patients with alcoholic cirrhosis 


\subsection{Procedures}

TIPSS was performed successfully in 28 of the 31 (90.3\%) patients randomised. Failure in the 2 patients was due to an inability to puncture the portal vein. These patients were managed successfully by VBL. The third patient was found to have a thrombosed portal vein at the time of insertion of TIPSS and this patient was managed successfully with oesophageal transection. Mean time to the performance of TIPSS from the time of randomisation was $2.2( \pm 0.2)$ days. Successful TIPSS was followed by a reduction in the PPG to less than $12 \mathrm{mmHg}$ in all patients undergoing successful procedures. Perforation of the capsule of the liver occurred in 1 patient and respiratory depression due to the sedation in another. The former patient died and the latter responded quickly to the reversal of sedation using the benzodiazepine antagonist, flumazenil.

VBL was successfully performed in all patients. Mean time from randomisation to VBL was $2.4( \pm 0.2)$ days. Varices were successfully eradicated in 18 patients ( 3 patients died before eradication, 6 patients had TIPSS for uncontrolled variceal rebleeding before variceal eradication). Mean number of sessions for eradication of varices was $3.1( \pm 0.4)$. Complications related directly to endoscopic therapy were oesophageal ulceration in 12 patients and aspiration pneumonia in 2. A total of 83 endoscopic sessions were performed in patients in the VBL group during the period of the study. Eight endoscopic sessions were required for variceal eradication in the 2 patients in whom the insertion of TIPSS was unsuccessful. 


\subsection{Rebleeding}

Four episodes of rebleeding from oesophageal varices occurred in 3 patients $(9.8 \%)$ in the TIPSS group. Variceal rebleeding was associated in all patients with significant shunt insufficiency due to shunt thrombosis in 2 (days 2 and 3 after TIPSS). PPG in both these patients increased from 9 and $10 \mathrm{mmHg}$ immediately after TIPSS to 14 and $21 \mathrm{mmHg}$. The shunts were balloon angioplastied with successful reduction in the PPG to less than $12 \mathrm{mmHg}$ in both patients. Both these patients had Child class $C$ disease. The third patient rebled before TIPSS was inserted (2 days after randomisation). One further patient rebled from a duodenal ulcer. No deaths occurred within 6 weeks of the rebleed.

Twenty two episodes of variceal rebleeding occurred in 14 of the 27 patients in the VBL group (51.9\%). One further patient rebled from a sclerotherapy ulcer. Rebleeding in 12 patients occurred before variceal eradication. One patient rebled from recurrence of oesophageal varices and 1 from gastric varices. Seven rebled in the first week of VBL, 3 in the second week, 2 in the third week and 1 after 7 months and 1 after 14 months. Frequency of variceal rebleeding was significantly higher in patients with Child class $C$ disease compared with those having Child class $A$ and $B$ disease $(\mathrm{p}-0.02)$. Rebleeding in the VBL group was uncontrolled in 8 patients (oesophageal varices-7, gastric varices-1). These patients required emergency TIPSS treatment. Four of the 14 patients who rebled from varices died within 6 weeks of the episode of rebleeding. Two of these patients died 
despite adequate control of bleeding with TIPSS. The other six patients who were treated with TIPSS have had no further rebleeding.

Variceal rebleeding was significantly lower in patients in the TIPSS group $(p<0.0006)$ (Figure 4.3$)$. The frequency of rebleeding remained significantly elevated in patients in the VBL group when sub-group analysis was performed to compare the Child class A and B patients $(<0.004)$, and Child class $C$ patients $(p<0.004)$ separately between the two groups.

Overall, the absolute reduction in risk of rebleeding with TIPSS was 0.4 and the relative reduction in the risk of rebleeding was 0.8 . This implies that if 2.4 patients with variceal haemorrhage are treated with TIPSS instead of VBL, 1 episode of variceal rebleed would be prevented.

\subsection{Mortality}

Thirteen patients $(42.2 \%)$ died in the TIPSS group. Six of these patients died early $(19.7 \%)$. The deaths were related liver failure in 3 , to the procedure in 1 (intraperitoneal bleeding from puncture of the liver capsule), cerebrovascular accident in 1, aspiration pneumonia and sepsis in 1. Seven patients (22.6\%) died during follow up, and these deaths were due to end-stage liver failure in 4, cerebrovascular accident in 1, cardiomyopathy in 1 and pancreatic neoplasm in 1. Patients with Child class $\mathrm{C}$ disease were at a significantly higher risk of dying during the period of follow up compared with patients with Child class A or B disease $(p=0.01)$.

Ten patients (36.9\%) died in the VBL group. Three patients died early (11.1\%). These deaths were related to aspiration pneumonia and sepsis in 1 , 
and variceal rebleeding in 2 . Seven other patients $(25.8 \%)$ died during follow up. Deaths were due to end-stage liver disease in 3 , variceal rebleeding in 2 , pneumonia and respiratory failure in 1 and myocardial infarction in 1 . Within group analysis showed that patients with Child class $\mathrm{C}$ disease were at a significantly higher risk of dying compared with patients with Child A or B disease $(p=0.005)$.

Mortality was not significantly different between the two groups (Fig 4.4). Sub-group analysis comparing mortality between Child class $A$ \& $B$, and Child class C patients, between the two groups showed no significant differences ( $p=0.23$ and 0.68 respectively).

\subsection{Encephalopathy}

Seven patients $(25.8 \%)$ were encephalopathic before TIPSS. Three had grade II and four had grade III encephalopathy. Variceal bleeding was the first presentation to the hospital in 2 patients with grade II encephalopathy and 1 patients with grade III encephalopathy who were well upto the time of presentation. Following insertion of TIPSS and control of bleeding there was a transient improvement in encephalopathy in 2 of these patients. The third patient improved with medical measures but has had episodes of spontaneous encephalopathy during follow up. The other 4 patients were known to suffer from chronic liver disease and had been diagnosed in the past to have chronic relapsing encephalopathy. Worsening in the severity of encephalopathy occurred in 1 patient. Development of the first episode of encephalopathy that was previously absent occurred in 4 patients (total, 
$16.1 \%)$. A total of $11(35.5 \%)$ patients were encephalopathic after TIPSS. Encephalopathy in 3 of these 4 patients was most marked immediately after TIPSS. Encephalopathy became clinically evident 5 months after TIPSS in the other patient. All episodes of encephalopathy responded to conservative treatment with lactulose and protein restriction. One patient with recurrent, spontaneous encephalopathy did however, require reduction in the shunt size with the reducing stent (Angiomed, UK). This increased the PPG from 1 $\mathrm{mmHg}$ to $9 \mathrm{mmHg}$ and this together with conservative measures allowed the patient to be discharged home.

Nine patients $(33.3 \%)$ in the VBL group were encephalopathic at randomisation. Four had grade II and 5 had grade III encephalopathy. Variceal bleeding was the first presentation to the hospital in 3 patients with grade II encephalopathy and 2 patients with grade III encephalopathy who were well upto the time of presentation. Following control of bleeding and medical measures, significant improvement was noted in 3 of these 5 patients. The other four patients with encephalopathy were known to suffer from chronic decompensated liver disease with recurrent encephalopathy. Three other patients (11.1\%) became encephalopathic during follow up. One of these three patients had TIPSS inserted for uncontrolled variceal haemorrhage. All episodes of encephalopathy were managed successfully with conservative measures. Encephalopathy in these 3 patients occurred after 3 weeks, 6 weeks and 8 months of randomisation. There was no significant difference in frequency of development of encephalopathy between the two groups. 


\subsection{Shunt Insufficiency}

Nine patients $(31.1 \%)$ in the TIPSS group developed shunt insufficiency (Figure 4.5). Shunt thrombosis occurred in 6 patients and was manifested by variceal rebleeding in 2 . The other 3 patients had pseudo-intimal hyperplasia which was diagnosed at routine portography. Portography was performed as an emergency for variceal rebleeding on two occasions when balloon angioplasty of the shunt was undertaken. Routine portography for shunt surveillance was performed on 49 occasions, with balloon angioplasty being performed for shunt insufficiency on 17 occasions in 9 patients. One patient required insertion of a parallel stent for recurrent shunt insufficiency. Two of the 8 patients in the VBL group who had TIPSS as 'rescue treatment' developed shunt insufficiency. One had shunt thrombosis and the other had intimal hyperplasia. Both were diagnosed at routine portography which was performed on 21 occasions in these 8 patients during the period of follow up.

\subsection{Sepsis}

Three patients $(9.7 \%)$ became septic during the first week of randomisation in the TIPSS group. One patient acquired an aspiration pneumonia and died, and 2 had staphylococcal septicaemia which responded to medical therapy. Four patients (14.7\%) in the VBL group became septic within the first week of randomisation. Two patients acquired an aspiration pneumonia, 1 patient developed spontaneous bacterial peritonitis and 1 developed a central-line- 
related sepsis. One of the patients with aspiration pneumonia died, and others responded to medical treatment.

\subsection{Duration of Hospital Stay And Cost Of Treatment}

Patients in the TIPSS group spent a mean of $23.2( \pm 15)$ days per patient in the hospital during the period of follow up compared with a mean of 31.2 $( \pm 19)$ days per patient by the VBL group $(<0.05)$. Patients in the TIPSS group spent a mean of $0.2( \pm 0.8)$ days per patient in the intensive care unit compared with $1.15( \pm 2.3)$ days per patient in the VBL group $(p=0.03)$. Days per patient spent in the high dependency unit was $0.7( \pm 1.3)$ and $3.1( \pm 3.5)$ in the TIPSS and VBL groups respectively $(p<0.001)$. Patients in the VBL group who rebled required significantly greater time in the intensive care unit than the patients who did not rebleed $(p=0.02)$.

Details of the costs incurred in the two groups are summarised in Table 4.3. The total cost for procedures in the patients in the TIPSS group were $£ 2749$ per patient compared with $£ 1422$ per patient in the VBL group. The direct costs incurred from in hospital treatment was $£ 4020$ per patient in the TIPSS group compared with $£ 5782$ in the VBL group. Overall, it was $£ 1373$ per patient cheaper to treat patients with TIPSS compared with VBL. 


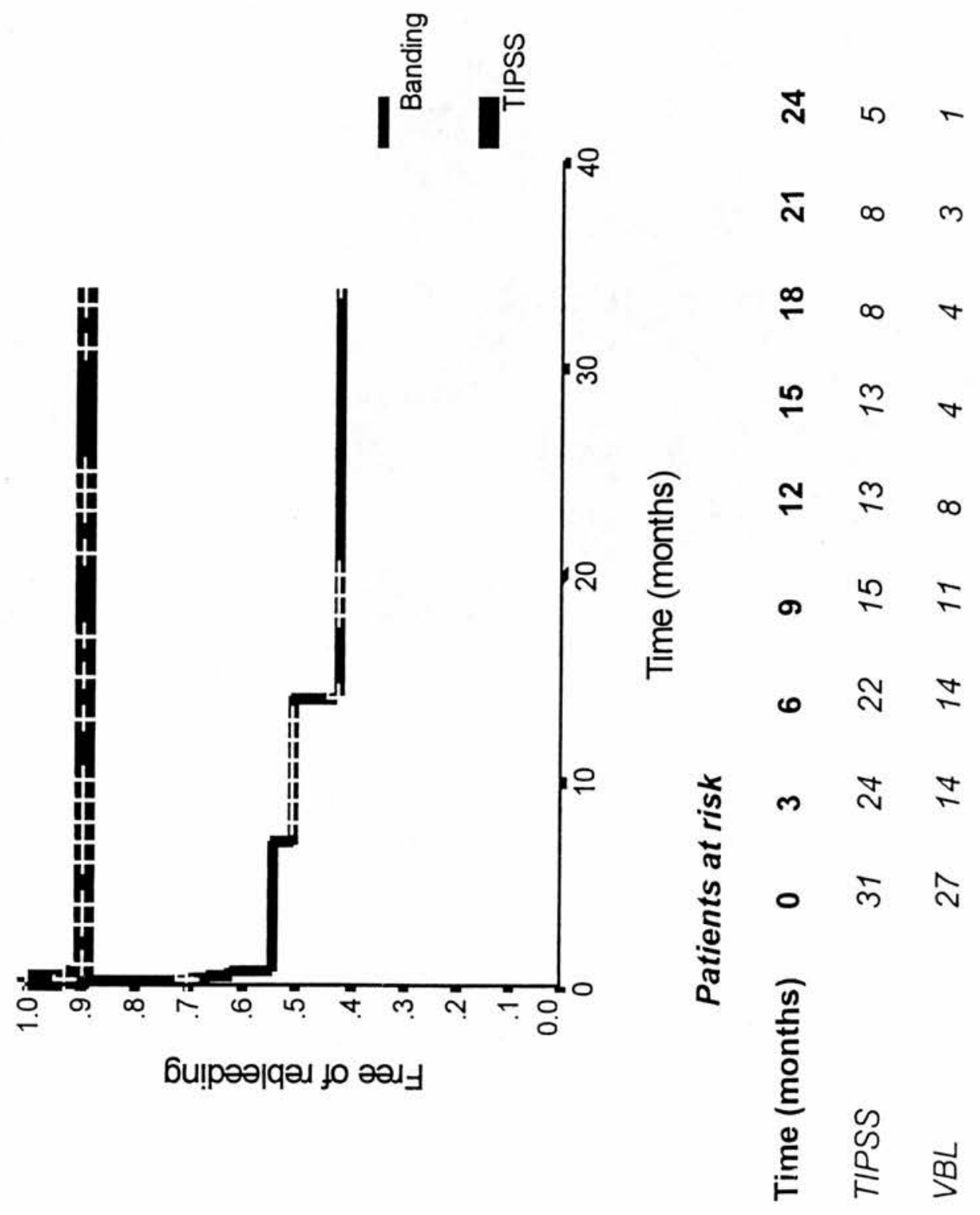



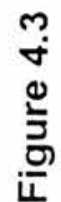




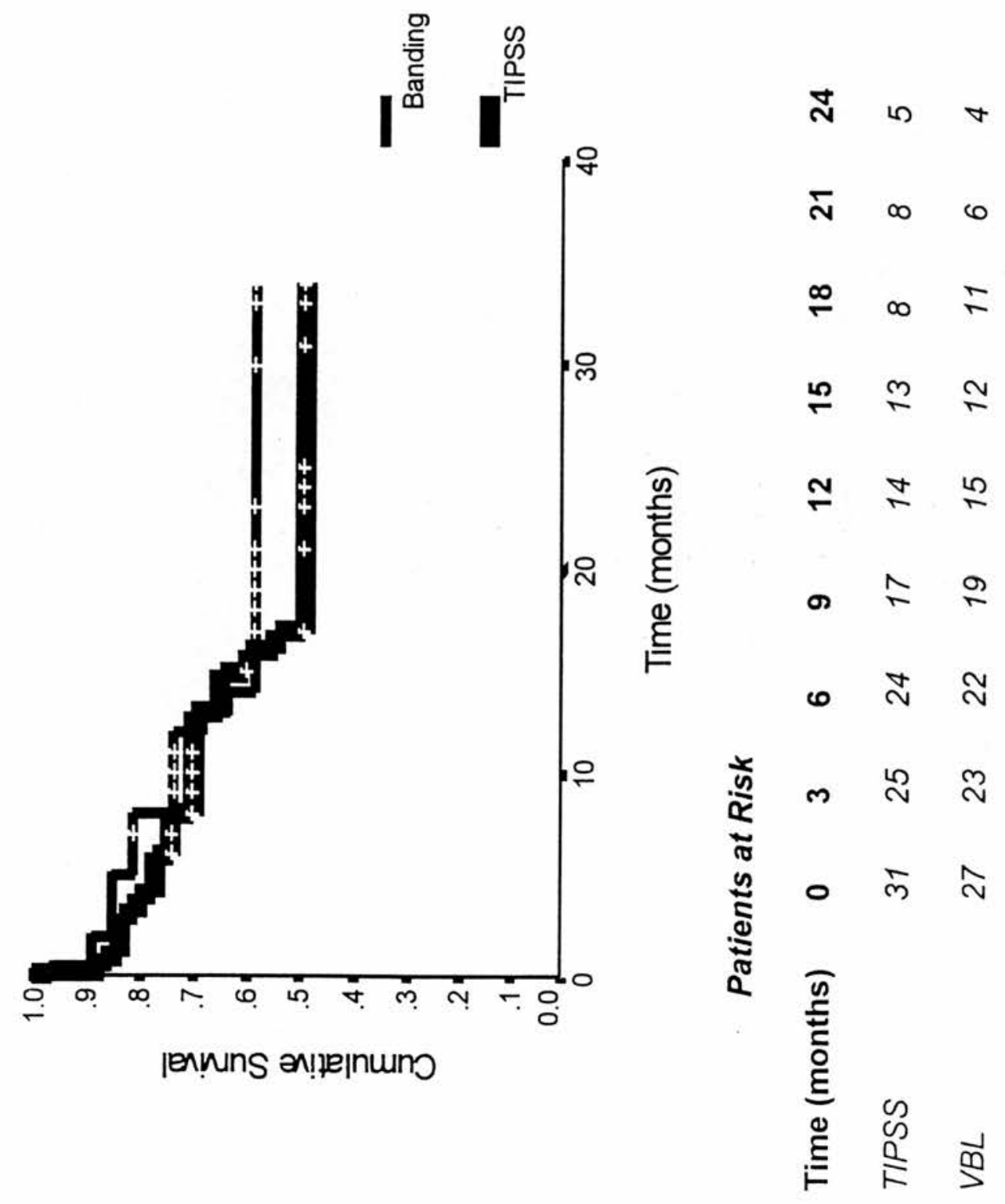



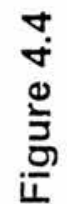




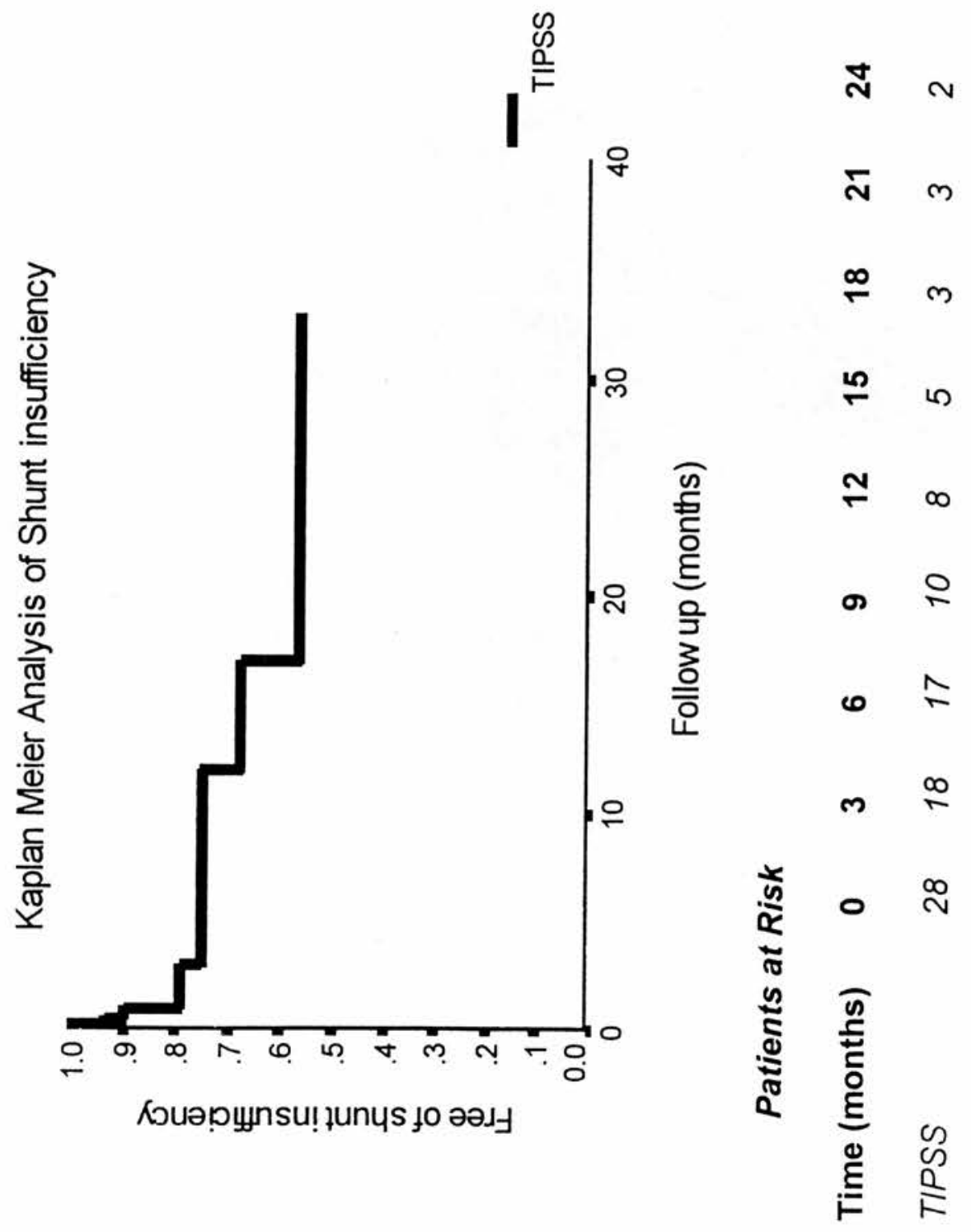




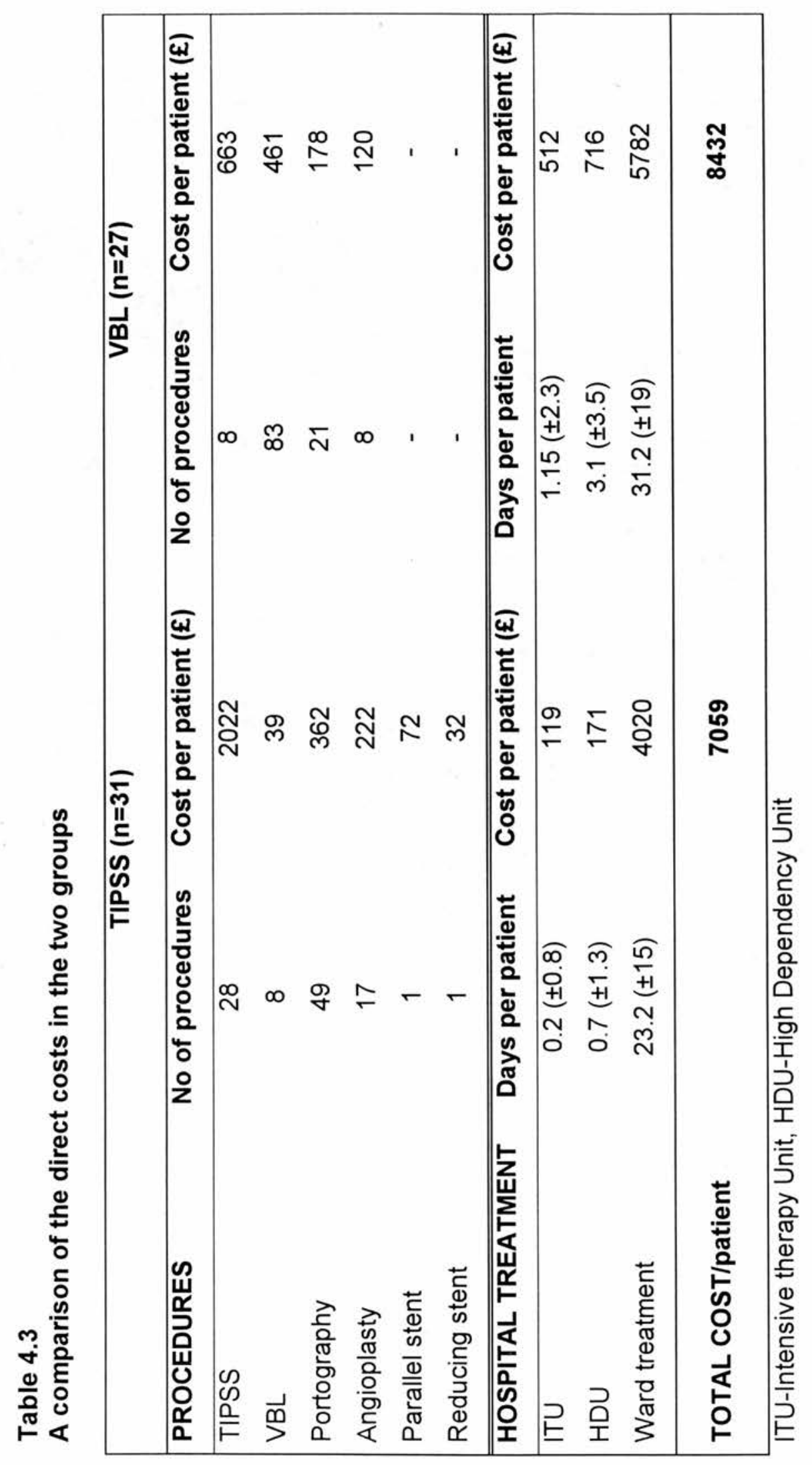




\subsection{SUMMARY}

In conclusion, if we take the primary end point as rebleeding, this study has shown that TIPSS is superior to variceal band ligation for the secondary prophylaxis of variceal haemorrhage in patients with cirrhosis. On the other hand, if mortality is taken as the revised end point then, the results of this study can also be interpreted to show the TIPSS is as effective as variceal band ligation plus 'TIPSS rescue'. It is important to remember that our trial compares TIPSS with variceal band ligation which has been shown to be superior to sclerotherapy for prevention of rebleeding. However, this study was designed to look at differences in rebleeding and not at mortality. This study has also shown significant reduction in the total cost of treating a patient with a variceal bleed in patients treated with TIPSS compared with VBL. However, this is the first study to show such a marked difference in rebleeding without other adverse complications and further studies are clearly indicated. 


\section{CHAPTER 5}

TIPSS as a Tool to Study the Renal

Dysfunction of Cirrhosis

- Sodium handling

- 'Hepatorenal reflex' 


\subsection{INTRODUCTION AND AIMS}

The spectrum of renal abnormalities in cirrhosis range from mild degrees of sodium retention to functional renal failure (hepatorenal syndrome). The factors leading to changes in renal circulation are intrarenal, neural and humoral. TIPSS reduces portal hypertension and has been reported to reduce ascites. Patients undergoing TIPSS for recurrent variceal haemorrhage or refractory ascites are haemodynamically stable and provide an opportunity to study the relationship between changes in portal pressure, renal handling of sodium and neurohumoral factors.

The aim of this study was to assess changes in the renal handling of sodium in patients with ascites following TIPSS and its relationship to changes in the neurohumoral profile (PRA, All ANP and cGMP), renal tubular function (LiCl) and the degree of portal hypertension. 


\subsection{METHODS}

\subsection{Patients}

Eighteen consecutive cirrhotic patients with ascites having TIPSS either for recurrent variceal haemorrhage (16 patients) (recurrent variceal haemorrhage was defined as bleeding requiring 3 or more admissions to the hospital whilst being treated using endoscopic means) or refractory ascites (2 patients) were studied. Patients were excluded from the study if they were haemodynamically unstable, had underlying malignancy, portal vein thrombosis, severe co-morbid diseases, biochemical evidence of renal dysfunction (serum creatinine of $>150 \mu \mathrm{mol} / \mathrm{l}$ ) or uncontrolled variceal haemorrhage (bleeding from varices despite 2 separate sessions of endoscopic treatment within five days). Patients were also excluded if they exhibited shunt insufficiency when portography and pressure measurements were performed at three months following insertion of TIPSS. Mean age was 52.5 years $( \pm 10.3)$ and 12 were males. Diagnosis of cirrhosis in all patients was made histologically. The cause of liver disease was alcohol related in 14, due to Hepatitis $C$ in 1 , primary biliary cirrhosis in 1 and cryptogenic in 2. Nine were Child class B and 9 were class $C$. All had ascites and their sodium intake was limited to $80 \mathrm{mmol} / \mathrm{day}$. All patients were on diuretics prior to TIPSS (mean spironolactone-180 mg $( \pm 24) /$ day and frusemide-60 mg $( \pm 12.6) /$ day $)$.

\subsection{TIPSS Procedure}


Details of TIPSS procedure are described in section 3.21 .

\subsection{Measurement Of Renal Function}

Plasma and urine were collected for $24 \mathrm{hr}$ before and after assessment of shunt function for the measurement of urinary volume, serum and urinary sodium and, serum and urinary creatinine. Creatinine clearance (Urinary creatinine $\times$ volume of urine $\times 24 /$ plasma creatinine $\times 60)(\mathrm{ml} / \mathrm{min})$ and sodium excretion (mmol/day) was calculated from the above data.

\subsection{Blood Sampling And Hormonal Assays}

About $40 \mathrm{ml}$ of blood was collected from a peripherally sited cannula into precooled tubes prior to TIPSS and 3 months afterwards when the patients attended for portography. Blood was collected before the patients arose in the morning and were in the fasted state. All patients were haemodynamically stable at the time of collection of the blood at not receiving concurrent infusions or transfusions. Plasma was separated and the samples stored at $-70^{\circ} \mathrm{C}$ for analysis at a later date.

5.1241. Measurement of Plasma renin activity (PRA): $5 \mathrm{ml}$ blood was collected into tubes containing $100 \mu \mathrm{l}$ of $5 \%$ aqueous di-sodium salt of ethylene diamine tetra acetic acid. Plasma stored at -70 degrees $\mathrm{C}$ were thawed at room temperature. The assay is based on the principle that Angiotensin $\mathrm{I}$ is generated by the action of renin on its substrate angiotensinogen. This is carried out at 37 degrees $C$ for 1 hour following 
which the reaction is arrested by sudden cooling. The protease inhibitors prevent further degradation of Angiotensin I to Angiotensin II and III. The assay for Angiotensin I is based upon competition of radio-iodinated Angiotensin I or plasma Angiotensin I for binding the limited amount of antibody added. Intra-assay coefficient of variation was about $5.2 \%$ and the interassay coefficient of variation was $8.6 \%$. Normal range for PRA was 1.6 $\mathrm{ng} / \mathrm{ml} / \mathrm{hr}(1.5 \mathrm{SD})$ for patients on $80-100 \mathrm{mmol} / \mathrm{l}$ (no added salt diet) sodium intake per day and $3.2 \mathrm{ng} / \mathrm{ml} / \mathrm{hr}(1.1 \mathrm{SD})$ for patients on unrestricted intake.

5.1242. Measurement of Angiotensin II (AII): $2 \mathrm{ml}$ sample of blood was obtained in Angiotensin II inhibitor. Angiotensin II was extracted from $1 \mathrm{ml}$ plasma using an ethanol precipitation procedure. After centrifugation the supernatant was dried down under air at 37 degrees $C$ and the dried crystals resuspended in $0.5 \mathrm{ml}$ assay buffer. This was stored at -20 degrees $C$ until assayed. Angiotensin II values were then measured by radio-immunoassay with an in house rabbit antibody R6B4. Intra-assay coefficient of variation was $4.2 \%$ and the interassay coefficient of variation was $2.6 \%$. Reference range for All was $0.032( \pm 0.01)$.

5.1243. Measurement of Atrial natriuretic peptide (ANP): $5 \mathrm{ml}$ of blood was collected in tubes containing $100 \mu \mathrm{l}$ of $5 \%$ aqueous di-sodium salt of ethylene diamine tetra acetic acid and $50 \mathrm{ul}$ of Trasylol $(4000 \mathrm{IU} / \mathrm{ml})$. Plasma ANP was extracted in the Millipore 8 sample extraction unit and the radioimmunoassay performed using an antibody to human ANP that has been 
raised in the rabbit following immunization with human ANP coupled to bovine thyroglobulin by carbodi-imide. Intra-assay coefficient of variation was $6.9 \%$ and the interassay coefficient of variation was $9.8 \%$. Reference range for ANP was $62.1(( \pm 7.1) \mathrm{pg} / \mathrm{ml}$.

5.1244. Measurement of cyclic Guanosine monophosphate (cGMP): $2.5 \mathrm{ml}$ sample of blood was collected in tubes containing $50 \mu \mathrm{l}$ of $5 \%$ aqueous disodium salt of ethylene diamine tetra acetic acid. $0.5 \mathrm{ml}$ of the thawed sample was extracted for cGMP using an ethanol precipitation procedure. After centrifugation the supernatant was dried down under air at 37 degrees $\mathrm{C}$ and the dried crystals resuspended in $0.5 \mathrm{ml}$ assay buffer. This was stored at -20 degrees $C$ until assayed. The samples were then acetylated and the plasma cGMP values measured with an in - house antibody (ED2-3). Coefficient of variation for the assay was $3.6 \%$. Reference range of cGMP was $1.18 \mathrm{nmol} / \mathrm{l}( \pm 0.34)$.

\section{$\underline{5.125 \text { Measurement Of Lithium Clearance (Li Cl) }}$}

$\mathrm{Li} \mathrm{Cl}$ was measured prior to and following insertion of TIPSS. $\mathrm{LiCl}$ was calculated over a 6 hour period. Following ingestion of $300 \mathrm{mg}$ of Lithium carbonate $12 \mathrm{hr}$ before, plasma and urine were collected at hourly intervals for 6 hours. Patients drank about $1.5 \mathrm{~L}$ of fluids prior to and during the study. Urine volume was measured and recorded hourly. Concentration of Lithium were measured in the plasma and urine using flame photometry. Clearance 
was calculated for every hour as described for creatinine above and averaged over 6 hours.

\subsection{Analysis}

Results were expressed as mean and standard deviation unless otherwise specified. Difference within the group was calculated using paired t-test and the relationship between variables calculated using linear regression. Data for changes in the various parameters in individual patients are shown with symbols in the figures. 


\subsection{RESULTS}

\subsection{Patients}

Bleeding was successfully controlled in the patients who had TIPSS for variceal haemorrhage. Mean PPG prior to TIPSS was $19( \pm 6) \mathrm{mmHg}$ and 8.8 $( \pm 3.4) \mathrm{mmHg}$ after shunt insertion. No clinically detectable ascites was present in any of the patients included in the study, although 4 still required diuretics. Mean dose of diuretics required for control of ascites in these 4 patients was $62.5 \mathrm{mg}( \pm 13)$ of spironolactone and $30 \mathrm{mg}( \pm 8.1)$ of Frusemide per day. Variceal rebleeding occurred in two patients due to shunt thrombosis within 1 week of the TIPSS and these were managed successfully by insertion of parallel stents. Two other patients had significant shunt dysfunction (pseudo-intimal hyperplasia) when routine portography was performed at 3 months and the mean PPG was $10.2( \pm 3.2) \mathrm{mmHg}$. Both patients having TIPSS for refractory ascites died during follow up from progressive liver failure 3.5 and 5.2 months after TIPSS and one other patient underwent orthotopic liver transplantation for intractable encephalopathy.

\subsection{Changes In Renal Function}

Urinary sodium concentration improved significantly from a mean of 39.3 $( \pm 6.3)$ to $69.6( \pm 7.9) \mathrm{mmol} / \mathrm{l}(p<0.001)$ (Figure 5.1) following TIPSS. Of the 2 patients having TIPSS for refractory ascites, sodium excretion improved from less than $5 \mathrm{mmol} / \mathrm{l}$ in both to 10 and $25 \mathrm{mmol} / \mathrm{l}$ respectively. There was also a 
significant improvement in the control of ascites in these patients, although they still required large amounts of diuretics. Creatinine clearance improved significantly from $46.6( \pm 9.7) \mathrm{ml} / \mathrm{min}$ to $93.5( \pm 11.6) \mathrm{ml} / \mathrm{min}$ following TIPSS $(p<0.0001)$ (Figure 5.2). Creatinine clearance in the 2 patients having TIPSS for refractory ascites improved from less than $5 \mathrm{ml} / \mathrm{min}$ before TIPSS to 12 and $15 \mathrm{ml} / \mathrm{min}$ after TIPSS.

\subsection{Change In The Neurohumoral Profile}

PRA decreased significantly from $13.4( \pm 3.8)$ to $4.5( \pm 1.5) \mathrm{ng} / \mathrm{ml} / \mathrm{hr}(p<0.007)$ (Figure 5.3). The levels were significantly higher than normal controls prior to TIPSS $(p<0.0001)$ and remained high after TIPSS $(P<0.01)$. All was significantly higher than controls before TIPSS $(p<0.0001)$ and remained high afterwards $(p<0.01)$. There was however, a significant reduction in its concentrations following TIPSS (pre TIPSS $-0.19( \pm 0.03) \mathrm{ng} / \mathrm{ml}$, post TIPSS - $0.07( \pm 0.01) \mathrm{ng} / \mathrm{ml}, \mathrm{p}<0.002)$ (Figure 5.4). Concentration of cGMP was significantly higher than controls prior to TIPSS $(p<0.001)$ and increased further from $2.7( \pm 0.3) \mathrm{nmol} / \mathrm{l}$ to $4(0.4) \mathrm{nmol} / /(\mathrm{p}<0.001)$ (Fig 5.5) following TIPSS. Concentration of ANP was not significantly different from the control population prior to TIPSS (control $-62.1( \pm 7.1) \mathrm{pg} / \mathrm{ml}$, patients $-83.7( \pm 17.2)$ $\mathrm{pg} / \mathrm{ml})$ and did not change significantly afterwards (90 $( \pm 18.7)(\mathrm{p}=\mathrm{ns})$ (Fig $5.6)$.

\subsection{Changes In Lithium Clearance}


Lithium clearance was measured in 8 of the patients with ascites who had TIPSS for recurrent variceal haemorrhage. There was a significant improvement in Lithium clearance following TIPSS from a mean of 19.4 $\mathrm{ml} / \mathrm{min}$ to $25.3 \mathrm{ml} / \mathrm{min}(\mathrm{p}<0.01)$ (Fig 5.7). The percentage change in Lithium clearance correlated with the percentage change in UNa $(r=0.52, p<0.05)$ and with the percent change in ANP $(r=0.54, p<0.05)$. There was no significant correlation between the change in $\mathrm{LiCl}$ and the change in $\mathrm{CrCl}$.

\subsection{Relationship Between Changes In Renal Function And The Severity}

\section{Of Liver Disease And The Change In PPG}

Although there was a significant improvement in the UNa and creatinine clearance following TIPSS which caused significant reduction in the PPG, no direct correlation was found between the change in PPG and the change in $\mathrm{UNa}$ and the change in creatinine clearance. Furthermore, the degree of change in $\mathrm{UNa}$ and $\mathrm{CrCl}$ was independent of the severity of underlying liver disease as assessed by the Pugh score. 


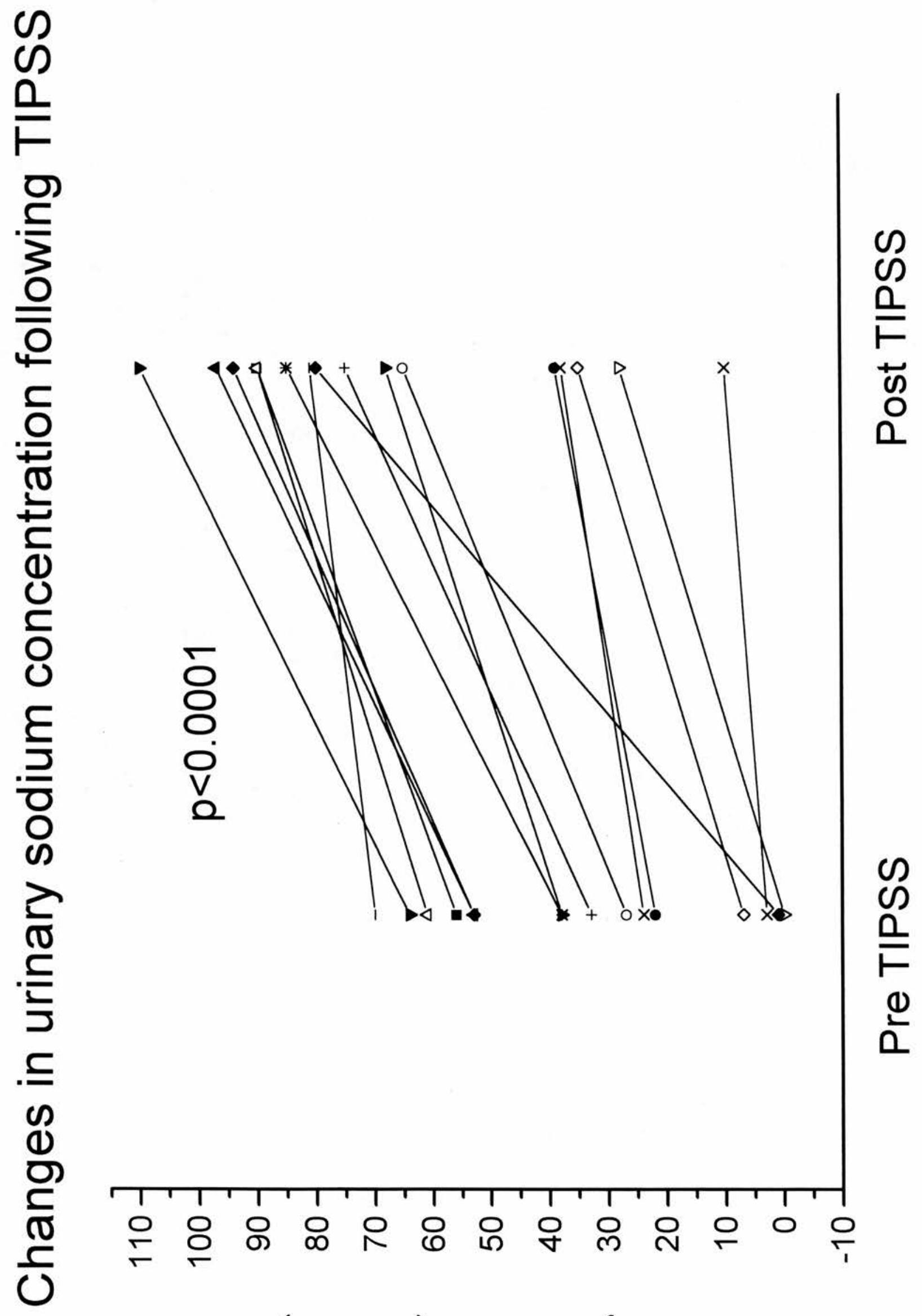

(I/lomm) mn!pos Kueu!un 


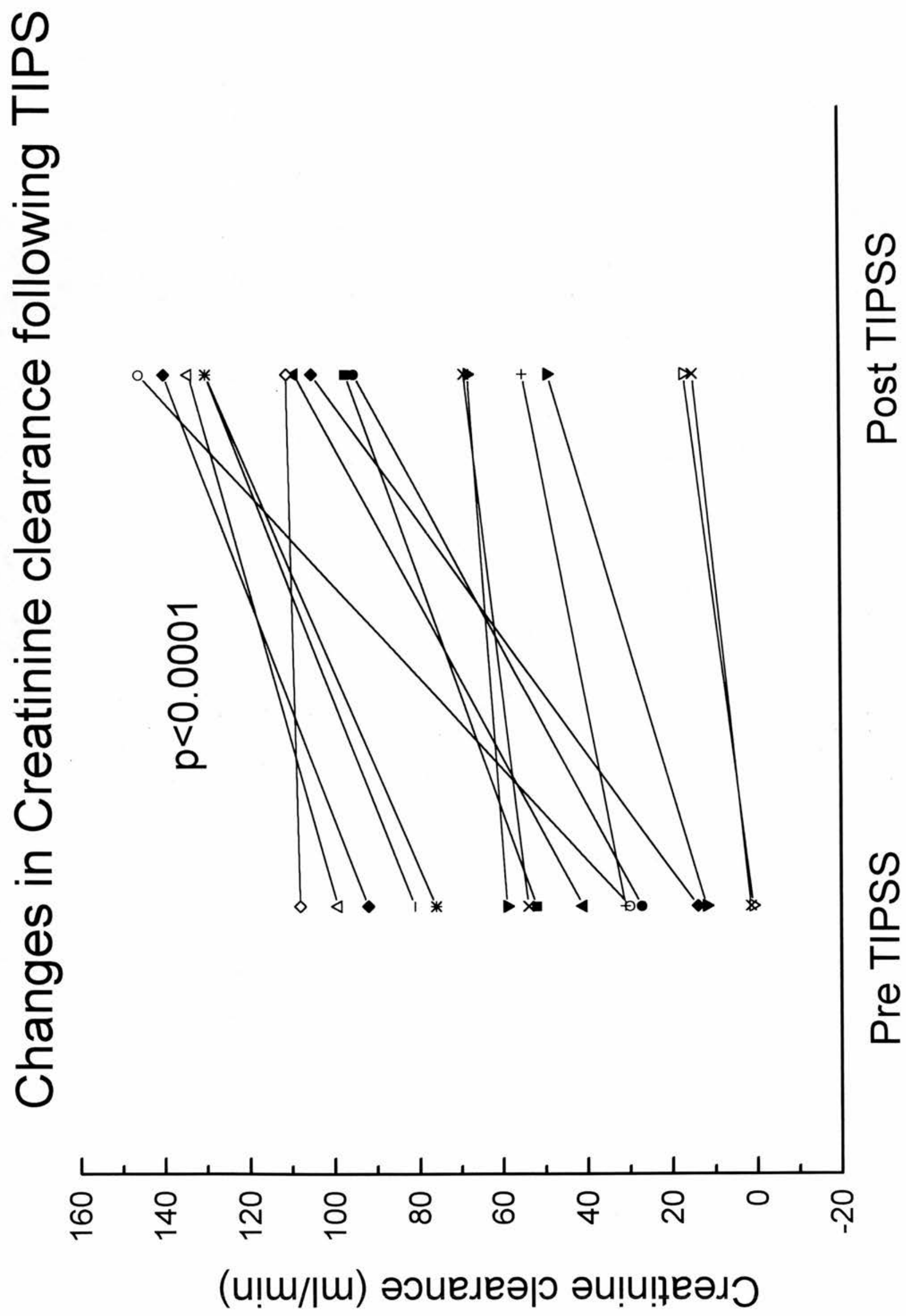



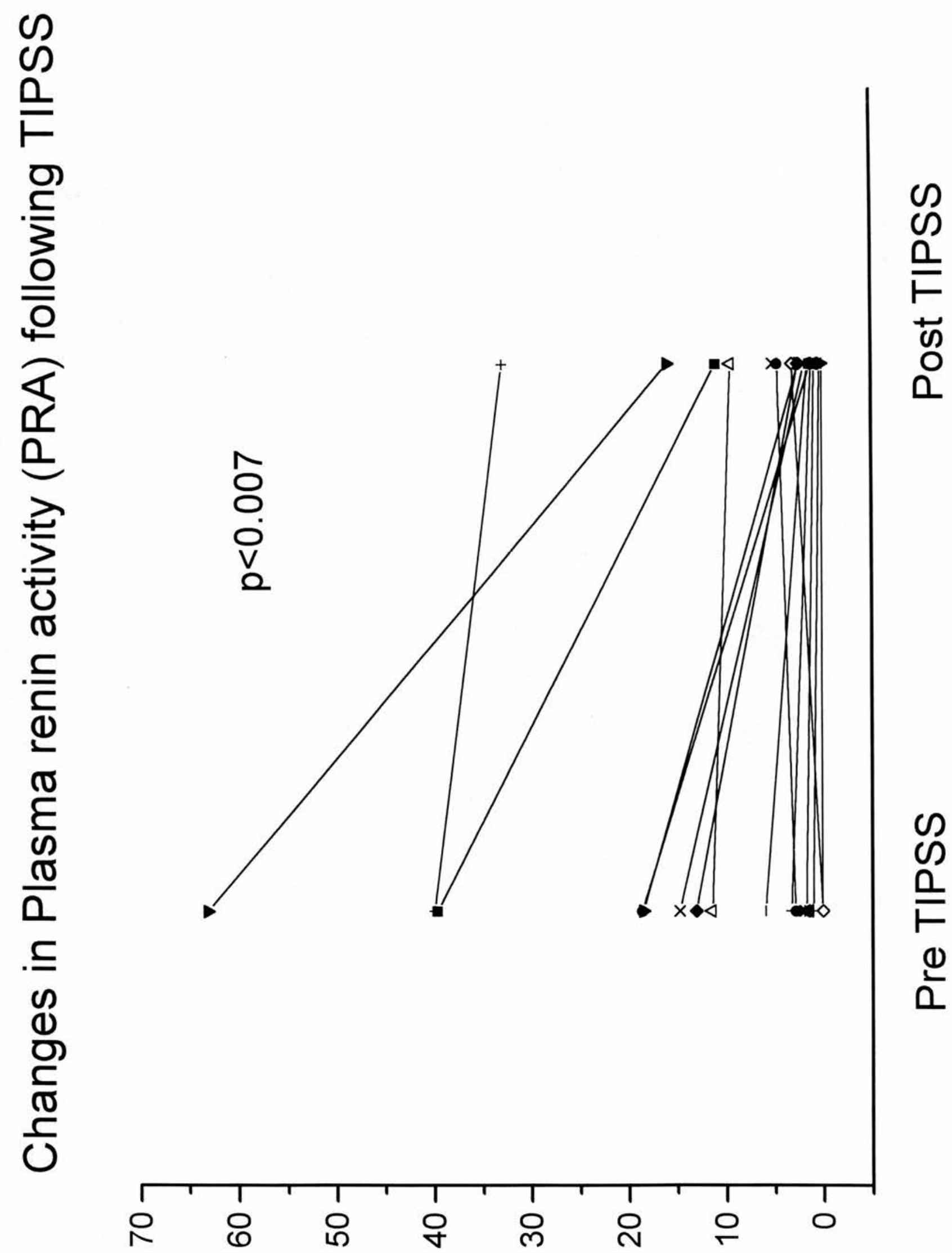

$(14 / 1 m / 6 u) \forall y d$

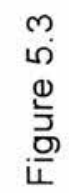



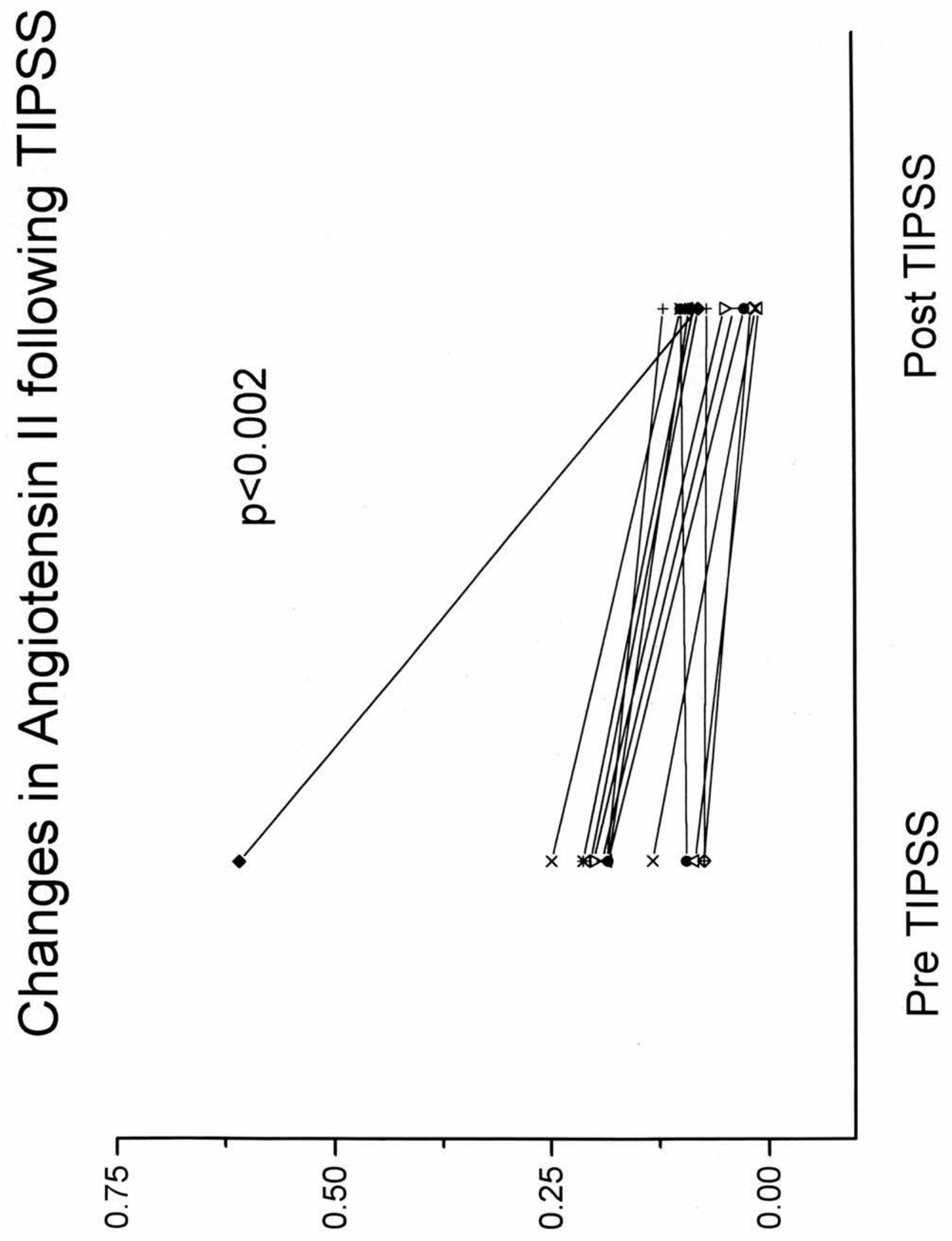

(ןu/bu) || u!suəło!̣u $\forall$

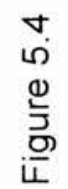




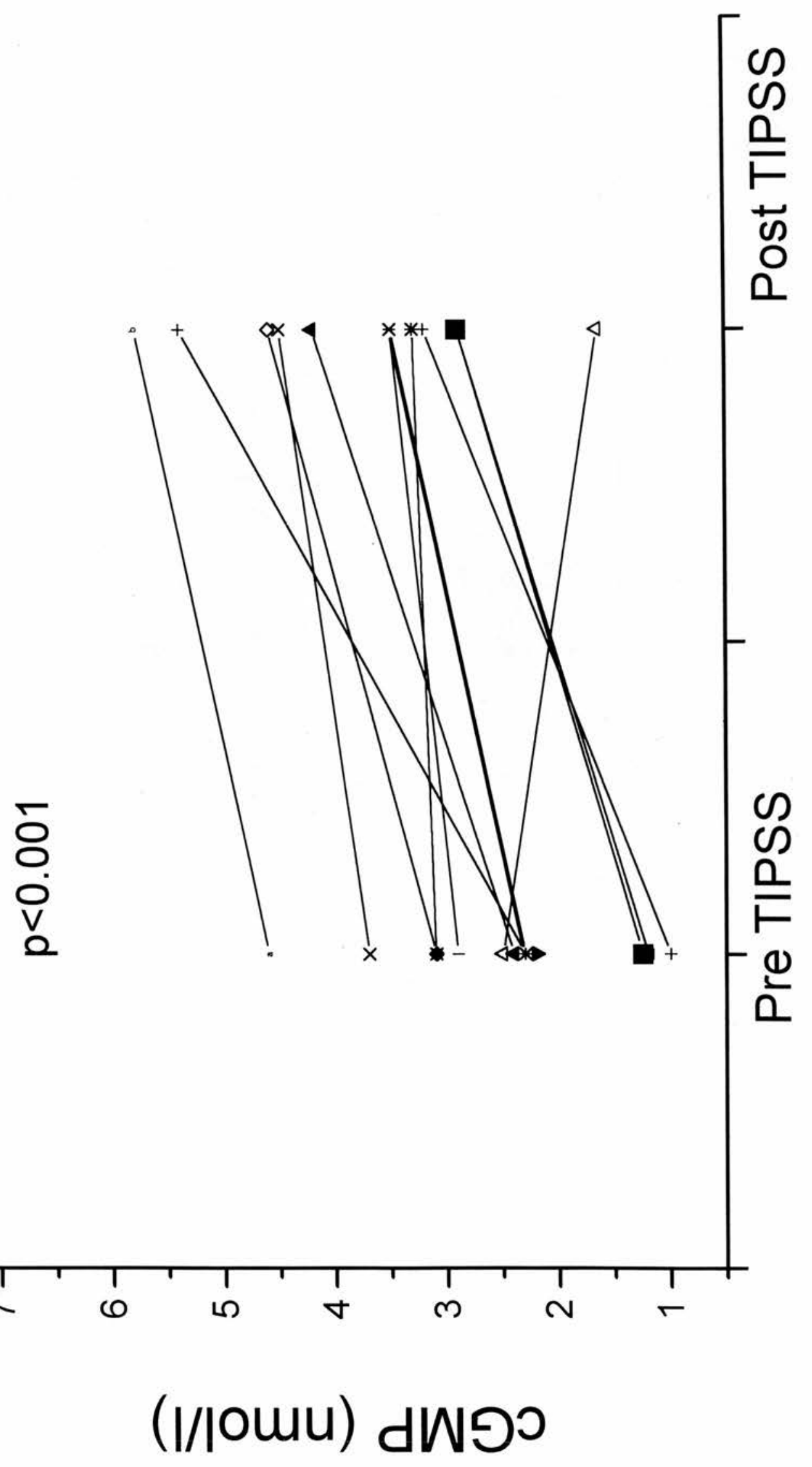




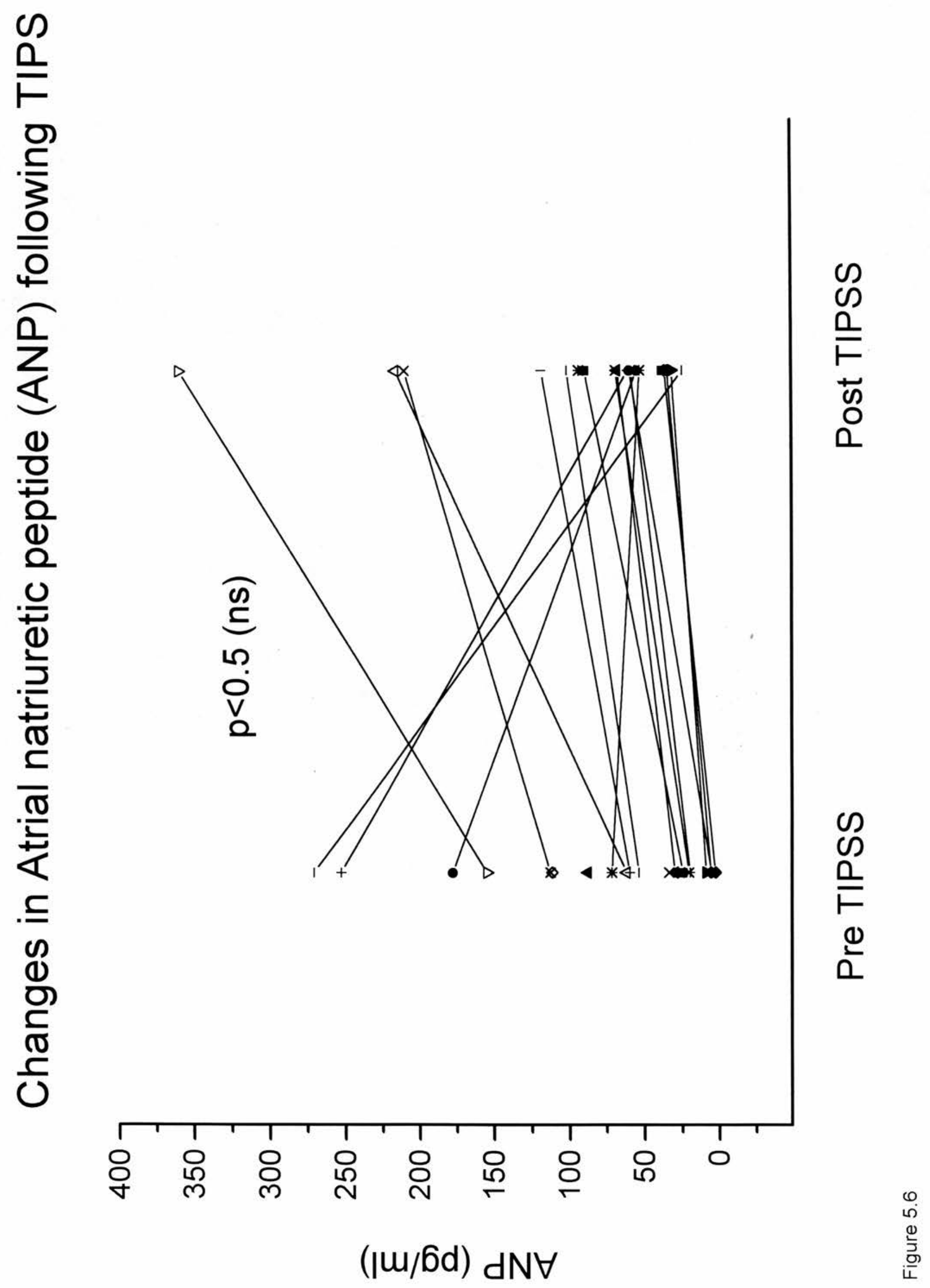




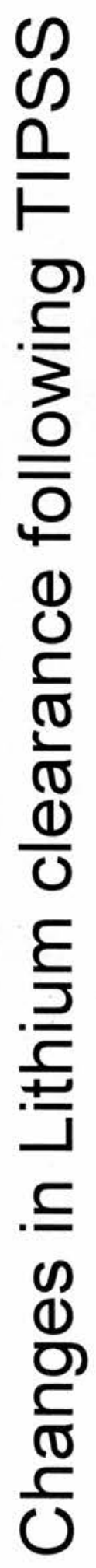

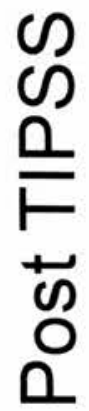

$\infty$

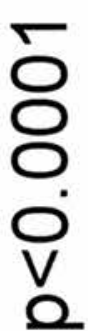




\subsection{SUMMARY}

The mechanism whereby sodium handling improves after TIPSS is multifactorial. This study has shown that although there is a significant reduction in PRA, All and PPG, none of these correlate with the changes in $\mathrm{UNa}$ and $\mathrm{CrCl}$. $\mathrm{LiCl}$ was used as a measure of sodium reabsorption proximal to the inner medullary collecting duct. cGMP measurements in this study probably reflects the activity of other unmeasured natriuretic factors and probably nitric oxide activity because the change in ANP which is the other major determinant of cGMP levels were both not significantly different prior to TIPSS and did not change significantly thereafter.

In conclusion, we have demonstrated that significant changes in $\mathrm{UNa}$ and $\mathrm{CrCl}$ after TIPSS is due both to changes in the renal proximal tubular function and a reduction in the neurohumoral factors that are involved in sodium retention. The degree of improvement in $\mathrm{UNa}$ and $\mathrm{CrCl}$ were independent of the severity of underlying liver disease and the change in PPG. 


\subsection{INTRODUCTION AND AIMS}

The characteristic circulatoryfinding in the renal dysfunction of cirrhosis is a reduction in renal blood flow (RBF) and an increase in renal resistance (Baldus et.al. 1970, Sacerdoti et.al. 1993). There is considerable evidence of enhanced sympathetic nervous activity in patients with cirrhosis and sodium retention. Concentrations of noradrenaline are inversely related to sodium excretion (Ring-Larsen et.al. 1982). Lang et.al. (1991), in an animal model, demonstrated an acute reduction in RBF following infusion of glutamine into the portal vein. The effect of the infusion was to induce acute hepatocyte swelling and portal hypertension. This reduction of RBF was abolished following section of the hepatic vagal fibres and renal denervation. He proposed the existence of a hepatorenal axis controlled by a reflex arc, the afferent limb of which was the hepatic vagal innervation and the efferent limb was the renal sympathetic system.

After insertion of TIPSS, portography is performed routinely to ensure adequate shunt function and this involves direct cannulation of the shunt. The shunt can be occluded using an angioplasty balloon producing significant increase in the portal PPG. The aim of this study was to investigate the 
relationship between changes in portal haemodynamics and renal blood flow in patients with cirrhosis. 


\subsection{METHODS}

The study was approved by the local ethics committee and the patients studied gave their informed consent. They were divided into 2 groups which were studied consecutively. Changes following shunt occlusion were measured either in RBF (group I) or in cardiovascular haemodynamics (group II).

\subsection{Patients}

Patients in the 2 groups were well matched for age, sex, aetiology and severity of liver disease. All patients had undergone TIPSS for recurrent variceal haemorrhage, 1-4 months previously. They were recruited into the study when they were attending for portography for the assessment of shunt function. None of them had ascites and were on no diuretics for at least 7 days prior to the study. They had no biochemical evidence of renal failure and their sodium intake was limited to $100 \mathrm{mmol} /$ day. They were included in the study only if they had no signs of shunt insufficiency at the time of portography which was defined as a Portal pressure gradient (portal pressure - inferior vena caval pressure) of less than $12 \mathrm{mmHg}$. Patient details are summarised on Table 5.1. 


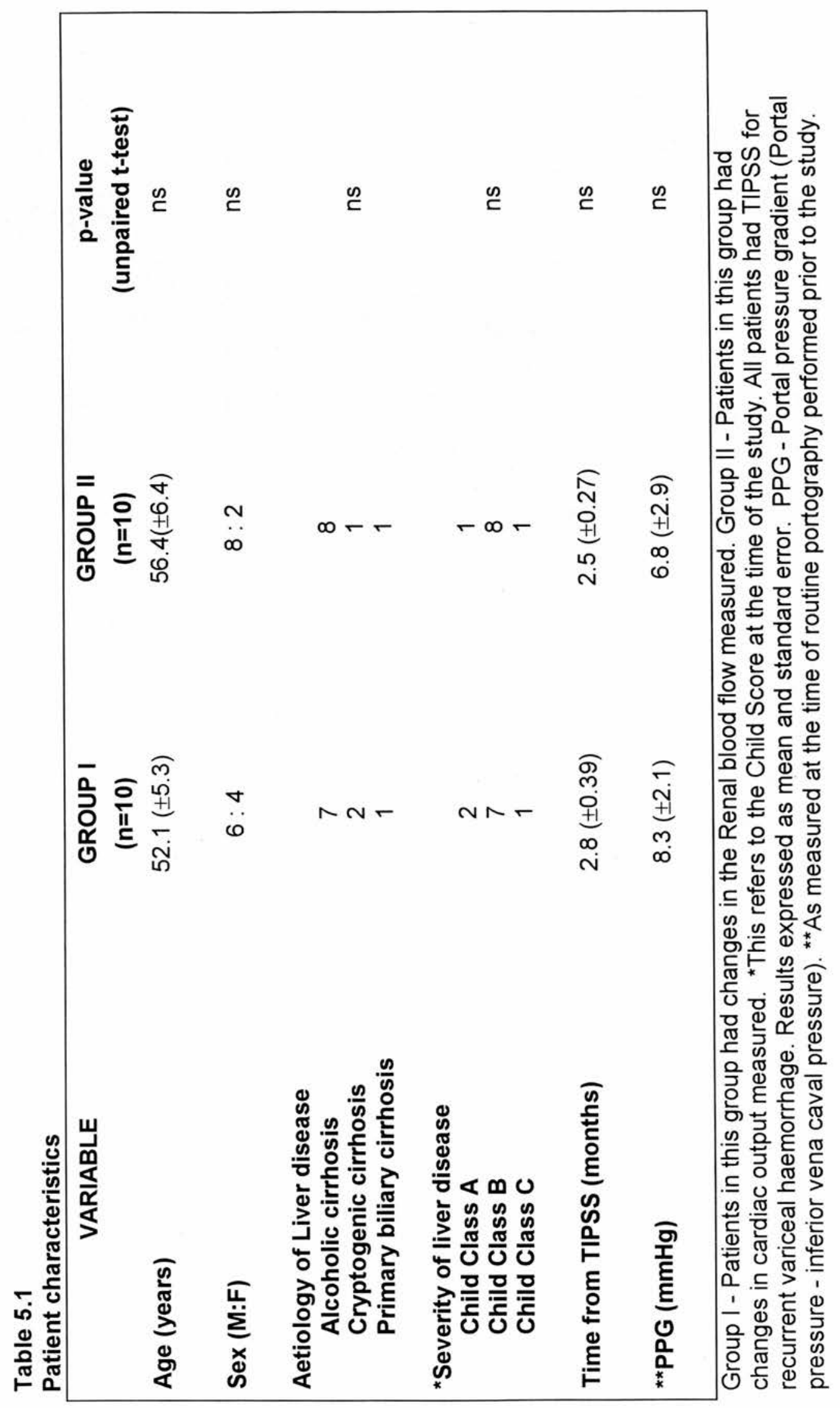




\subsection{PORTOGRAPHY}

Assessment of shunt function involved the catheterisation of the shunt via the internal jugular route using midazolam for sedation. Following portography to outline the portal system and the shunt, pressure was recorded (HewlettPackard, USA) from the portal vein, the inferior vena cava and the right atrium, and the portal pressure and porto-atrial gradient (PAG) were calculated. Unilateral RBF, renal vein pressure, portal vein flow and blood was sampled from patients in group I. Cardiac haemodynamics were studied in patients in group II. Measurement of other parameters as outlined in Table 5.2 were also made. 


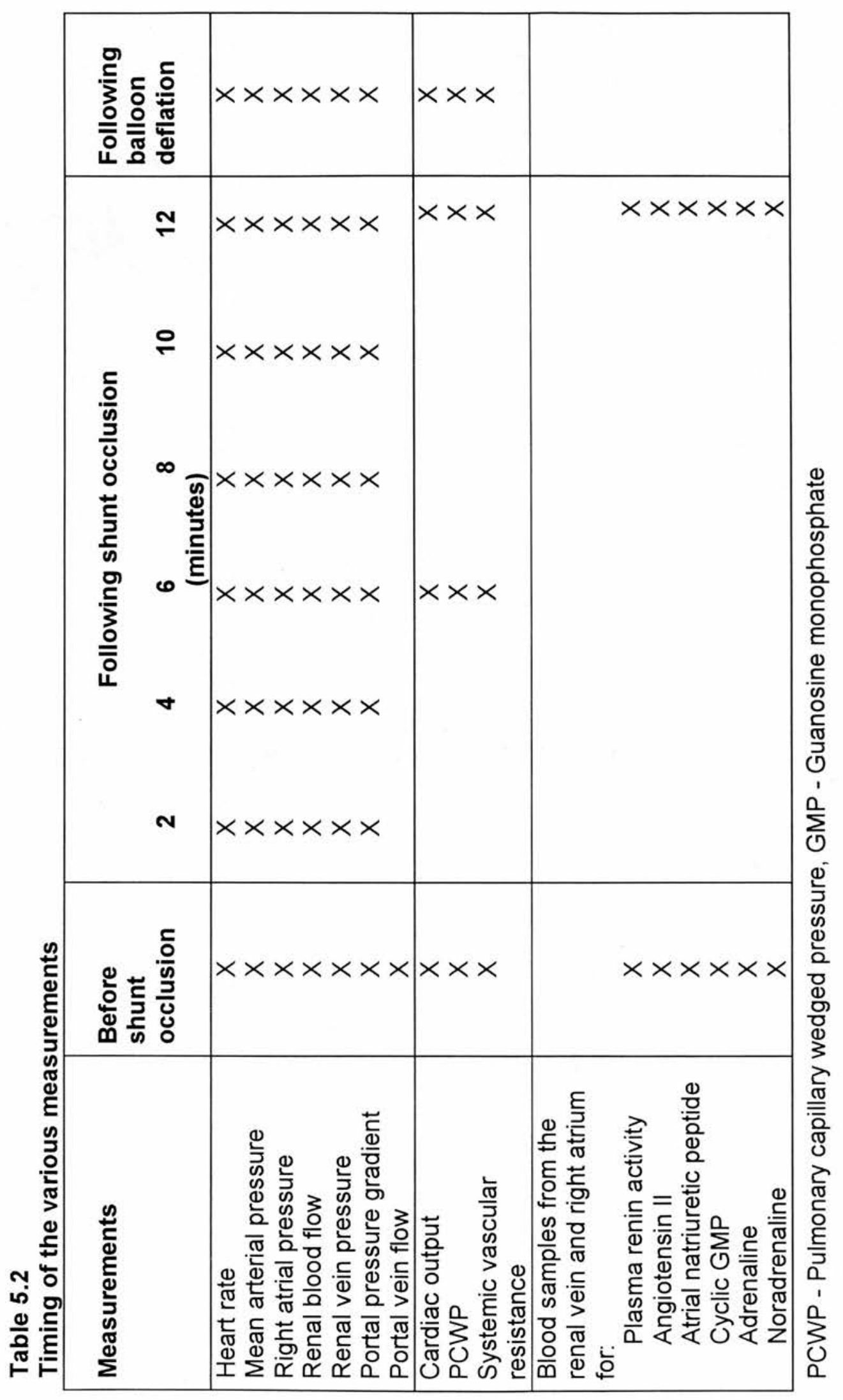




\subsection{Measurement Of Portal Vein Flow}

Following pressure measurements, the sheath was introduced through the shunt and a double thermistor catheter (Webster Laboratories, California) positioned in the portal vein. The distal end of this catheter was placed under fluoroscopic control in the portal vein just distal to the junction of the superior mesenteric and splenic veins. The protocol that was used for the measurement of changes in the portal and renal blood flow has been well validated by our group both in vitro and in vivo (Hayes et.al. 1992). Briefly, the injectate used was $5 \%$ Dextrose (at room temperature, about $22^{\circ} \mathrm{C}$ ). This was infused at $50 \mathrm{ml} / \mathrm{min}$ using a syringe pump (Harvard model 22, modified to meet BS 5724 standard). The signals from the internal (injectate) and external (mixed blood) thermistors (distance-2.4 cm) were transferred through a custom built interface (isolated to BS 5724 type CF) and processed in an IBM compatible PS2-286 microcomputer system (Figure 5.8). This provided a real time graphic display of blood flow. Collection of data was started and stopped as required at the different stages of the test. Mean blood flow was measured over 20 seconds. Specific events were designated using on screen markers. Data was stored on floppy discs for analysis at a later time.

\subsection{Measurement Of Changes In Renal Blood Flow}

Changes in unilateral RBF were measured in patients in group I. The catheter was replaced by an angioplasty balloon which was positioned in the intrahepatic portion (Figure 5.9) of the shunt. The sheath was positioned in 
the right atrium and the side arm used to measure the right atrial pressure. Electrocardiogram and pulse rate were monitored continuously. Mean arterial pressure was recorded non-invasively every 2 minutes. A double thermistor catheter was introduced through a sheath in the right femoral vein into the right renal vein and the changes in RBF and renal vein pressure continuously recorded as described for measurement of portal vein flow above. The right renal vein was chosen firstly, because the left renal vein also drains the suprarenal glands which would complicate both sampling of blood and measurement of blood flow and secondly, because cannulation of the right renal vein was a lot easier from the femoral vein as it follows the natural curvature of the catheter. Changes in the portal pressure were recorded through the central lumen of the angioplasty balloon. The coefficient of variation in RBF using this catheter was less than $10 \%$.

Following measurements at base-line the angioplasty balloon was inflated and the changes in RBF and other variables as outlined above recorded at 2 minute intervals for 12 minutes. The balloon was then deflated and the above parameters measured for 4 minutes at 2 minute intervals. Renal vein resistance was calculated as the ratio of the RBF and renal vein pressure.

\subsection{Measurement Of Cardiac Haemodynamics}

Changes in cardiac haemodynamics were measured in patients in group II. The outline of the procedure was similar to that used for the measurement of RBF. A Swan-Ganz catheter was introduced through a sheath in the femoral 
vein into the pulmonary artery and changes in cardiac output, systemic vascular resistance and pulmonary capillary wedge pressure recorded prior to, 6 and 12 minutes after shunt occlusion and, following balloon deflation. Three separate recordings were made and the average of these were recorded

\subsection{Blood Sampling And Hormonal Assays}

Plasma was collected from the right atrium and the renal vein respectively, prior to and $12 \mathrm{~min}$ after shunt occlusion and stored at $-70^{\circ} \mathrm{C}$ for analysis at a later date. Although, the pulmonary artery would have been ideal for sampling to prevent the effects of streaming and inadequate mixing of blood, the right atrium was chosen because it was thought unethical to introduce another catheter into the pulmonary artery.

Plasma was also collected from a peripherally sited cannula from 12 healthy volunteers with a mean age of $51.3( \pm 4.2)$, male/female $-8 / 4$ who were maintained on $80-100 \mathrm{mmol} \mathrm{Na}$ diet for 1 week.

Measurement of Plasma renin activity (PRA): See section 6.1241

Measurement of Angiotensin II (AlI): See section 6.1242

Measurement of Atrial natriuretic peptide (ANP): See section 6.1243 
Measurement of cyclic Guanosine monophosphate (cGMP): See section 6.1244

Measurement of Adrenaline and Noradrenaline: Extracted samples were assayed using high performance liquid chromatography and electrochemical detection (Goldstein et.al 1981). Coefficients of variation was $8.2 \%$ and the normal values for adrenaline and noradrenaline were less than 0.4 and 5 $\mathrm{nmol} / /$ respectively.

\subsection{Statistical Analysis}

Results were expressed as mean and standard deviation unless otherwise specified. Difference between groups was calculated using unpaired t-test. The relationship between variables were calculated using linear regression. The method of summary measures as described by Matthews et.al. (1990) was used to analyse the data. Briefly, a summary of the response (i.e. the rate of change in renal blood flow, cardiac output (Figures 5.10 and 5.11), arterial blood pressure, right atrial pressure) was calculated for every patient. The second stage was to analyse the summary measure using simple statistical technique such the paired t-test comparing the base-line value with the value at 12 minutes which was the point of maximal change. 
Fig 5.8 The computer set-up and the thermodilution system that was used to measure renal and portal blood flow.

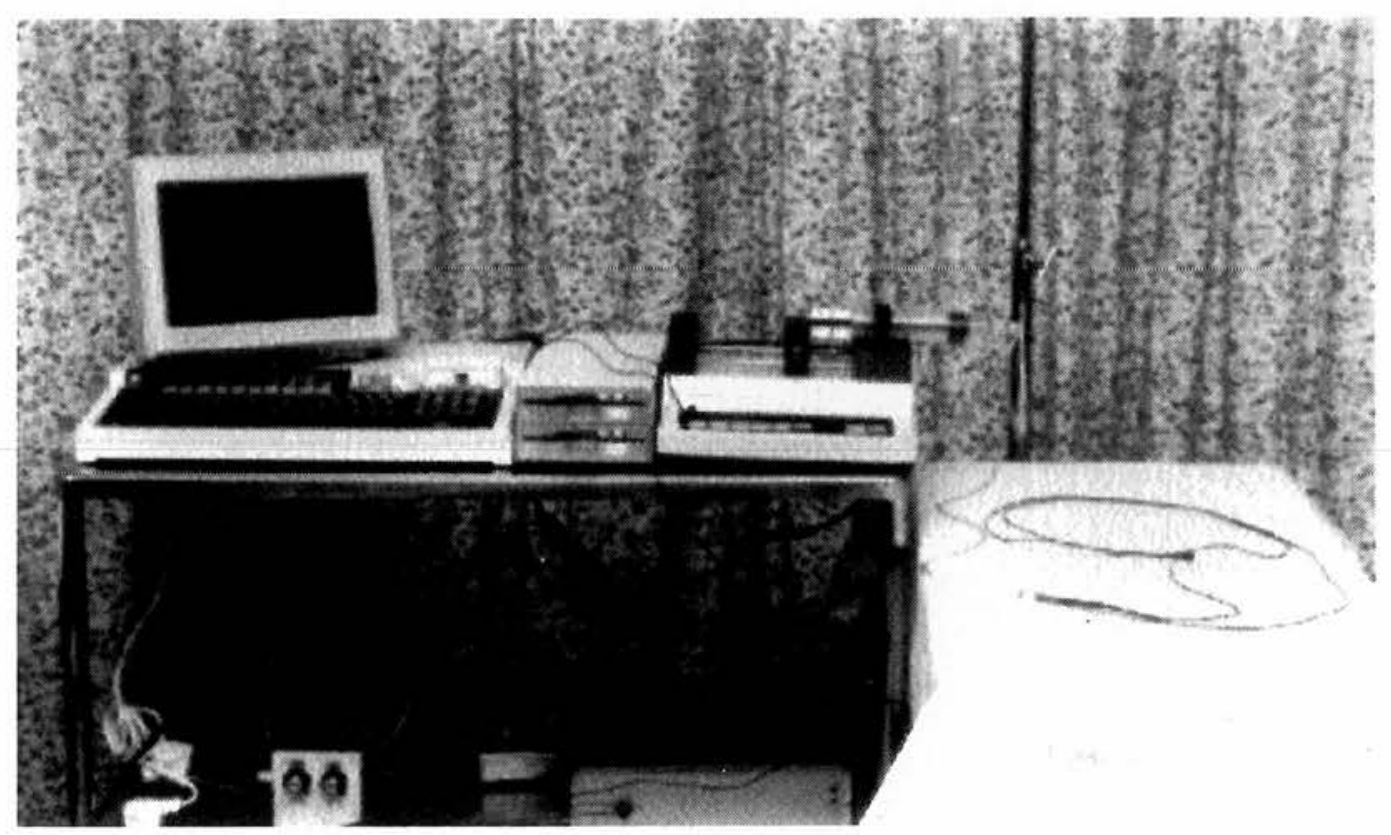


Fig 5.9 X-ray showing an inflated angioplasty balloon in the shunt inserted through the internal jugular vein and a reverse thermodilution catheter in the right renal vein inserted through the femoral vein.

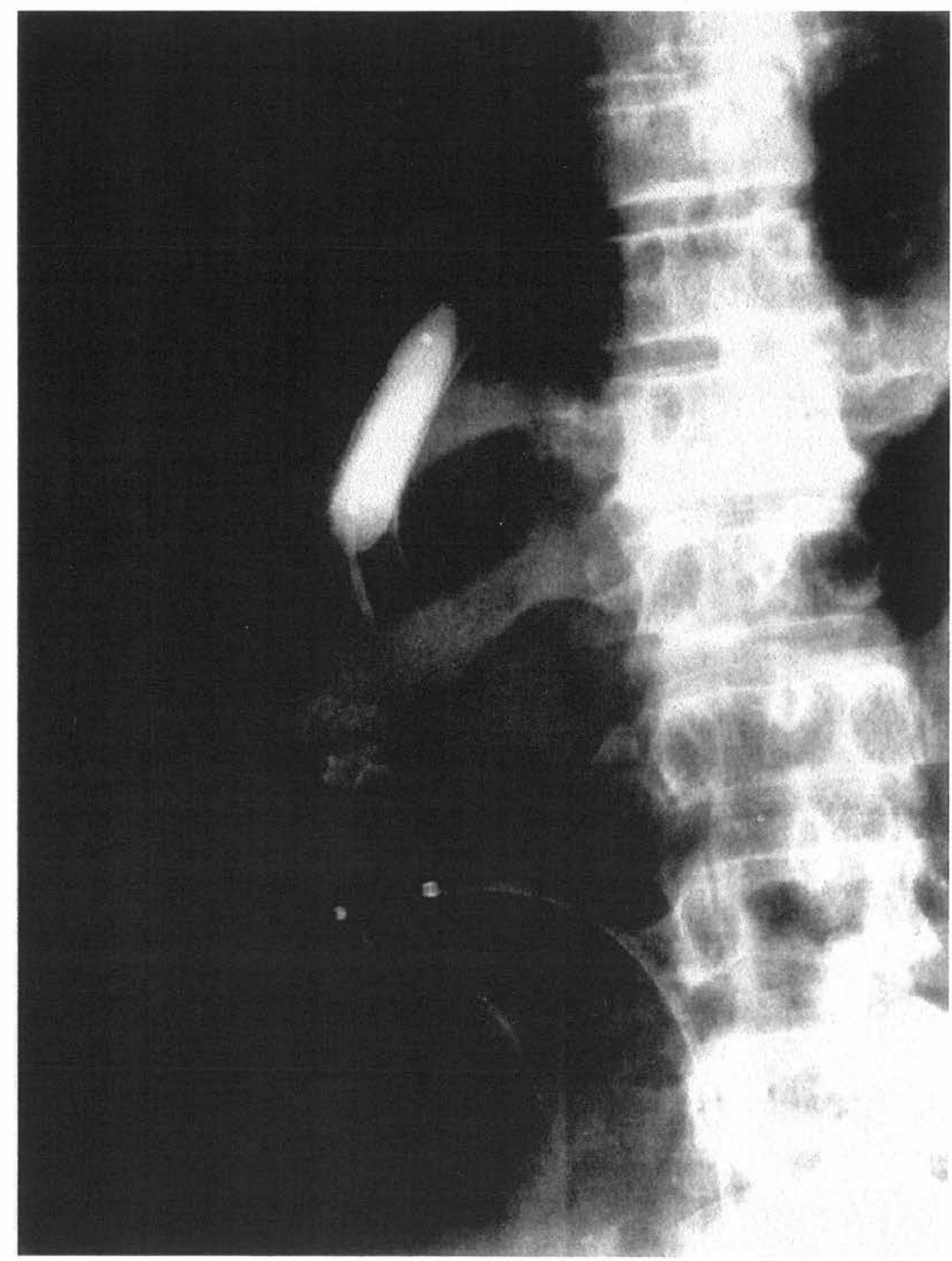




\subsection{RESULTS}

\subsection{PPG And Portal Vein Blood Flow}

The mean internal diameter of the shunt was $9.4 \mathrm{~mm}( \pm 1.3)$ and the mean PAG was $8.3( \pm 0.7) \mathrm{mmHg}$ representing an adequate shunt. Following shunt occlusion the PAG increased to $50( \pm 3.3)$. Following deflation of the balloon the PAG was $8.1( \pm 0.6) \mathrm{mmHg}$. Portal vein blood flow at rest was 482 $( \pm 201.6) \mathrm{ml} / \mathrm{min}$. No significant correlation was found between portal vein flow and the portal pressure gradient $(r=0.3, p=0.4)$. Resting RBF was 289 $( \pm 102.6) \mathrm{ml} / \mathrm{min}$ and this correlated inversely with portal vein blood flow ( $r=-$ $0.73, p<0.02$ ) (Figure 5.12).

\subsection{Changes in RBF}

Following shunt occlusion there was a progressive reduction in RBF $(P<0.001)$ which was most marked at 12 min (Figure 5.10) (Table 5.3). Mean change was $-133.8( \pm 89) \mathrm{ml} / \mathrm{min}(-43.5 \%( \pm 25.3)$. Following balloon deflation the RBF returned to base-line values over a period of 4 minutes. Shunt occlusion was associated with a significant increase in the PAG from a mean of $8.3( \pm 2.1)$ to $50.6( \pm 10.5) \mathrm{mmHg}(P<0.0001)$. The change in RBF correlated significantly with the change in PAG $(r=-0.85, p<0.001)$ (Figure 5.13).

Renal vein pressure decreased significantly following shunt occlusion $(-26 \%( \pm 17.2))(p<0.01)$. Although there was a mild reduction in heart rate $(-$ $6.3( \pm 7.4) / \mathrm{min},-7.8 \%( \pm 9.5))$, mean arterial pressure $(-10.3( \pm 9.4) \mathrm{mmHg},-$ 
$9.7 \%( \pm 8.6))$, and right atrial pressure $(-0.3( \pm 1.7) \mathrm{mmHg},-21.3 \%( \pm 74.2))$, these changes were not statistically significant (Table 5.4). Significant correlation was observed between changes in renal blood flow and the change in renal vascular resistance $(r=-0.88, p<0.0005)$. 


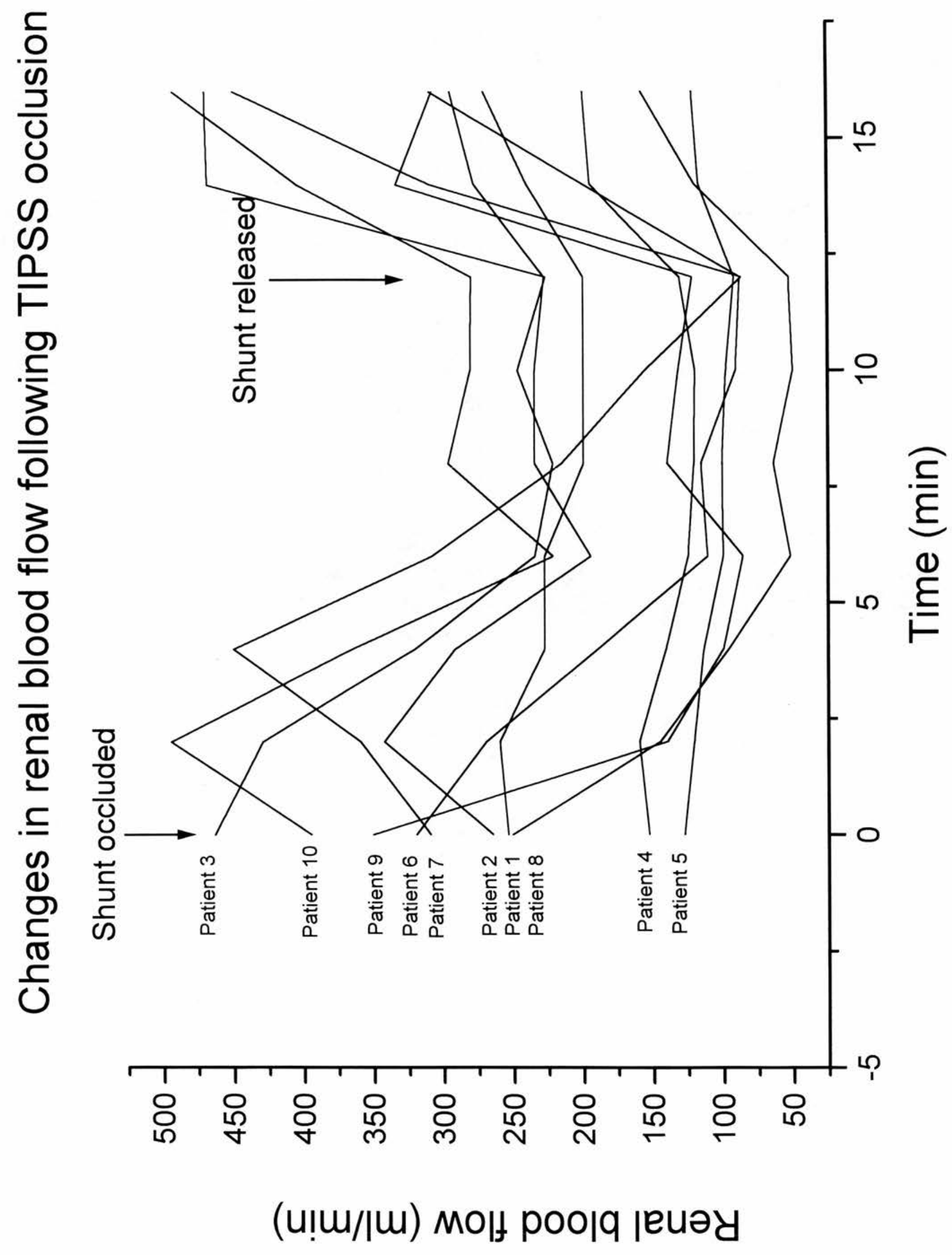




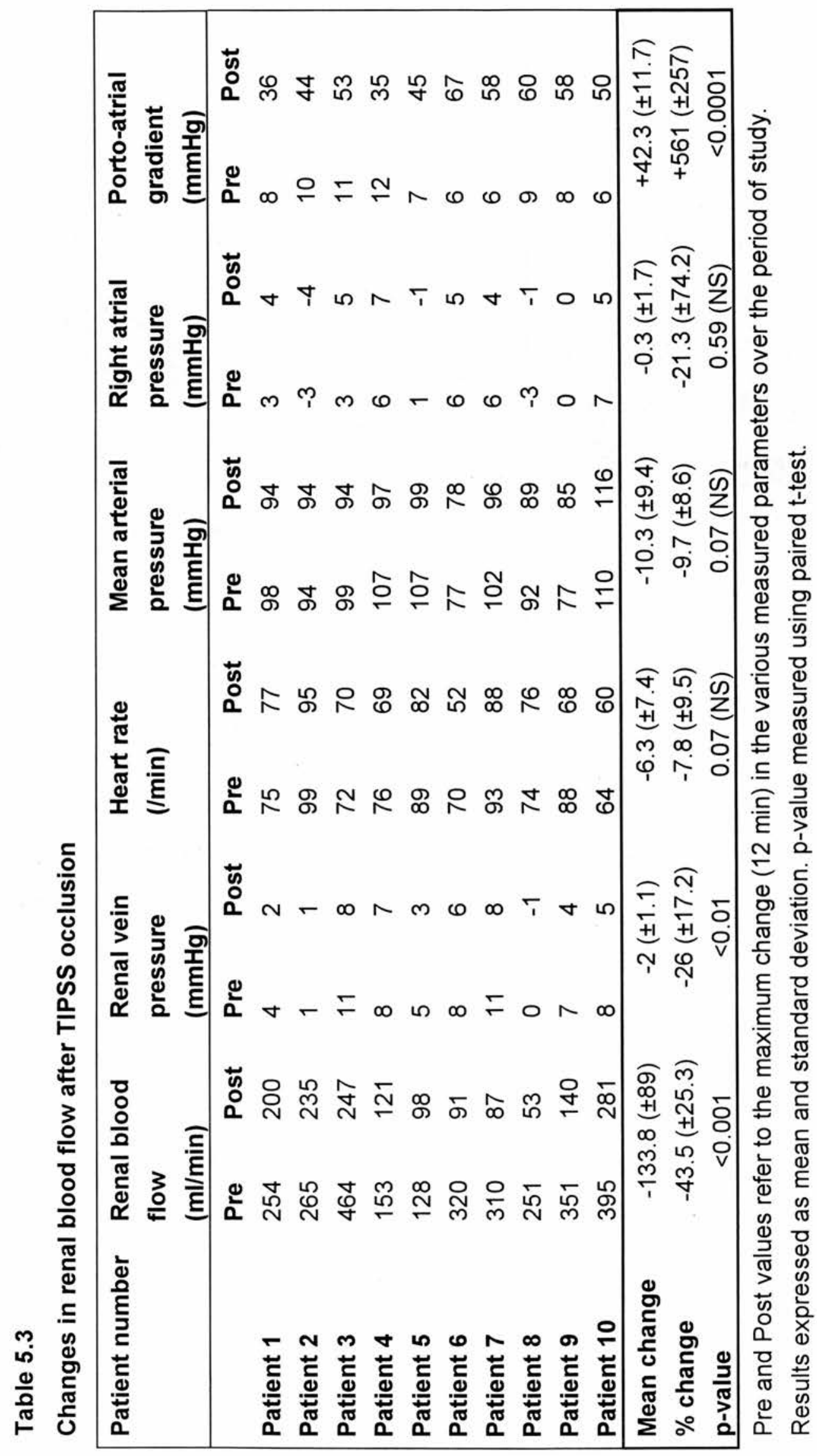




\subsection{Changes In Cardiac Haemodynamics}

Cardiac output was reduced from a mean of $7.4( \pm 1.3) \mathrm{l} / \mathrm{min}$ prior to shunt occlusion to $6.5( \pm 1) \mathrm{l} / \mathrm{min} 12 \mathrm{~min}$ after shunt occlusion $(p=0.02)$. This returned to pre-occlusion values following balloon deflation (Figure 5.12) (Table 5.4). Mean change was $-0.85( \pm 0.55) \mathrm{I} / \mathrm{min}(-10.9 \%( \pm 6.9))$. Shunt occlusion was associated with a significant increase in the PAG $(p<0.0001)$. This was not associated with any significant change in the heart rate, mean arterial pressure and the right atrial pressure. Shunt occlusion was associated with insignificant reduction in the pulmonary capillary wedge pressure $(p=0.06)$. Systemic vascular resistance increased significantly from $1128( \pm 270.6)$ to $1347( \pm 356.4)$ dyne $\mathrm{sec} / \mathrm{cm}^{5}(p=0.02)$. There was however, no significant correlation between the changes in the SVR and the changes in the cardiac output $(r=0.4, p=0.24)$. The change in cardiac output was also independent of the changes in the PAG $(r=0.5, p=0.09)$. 


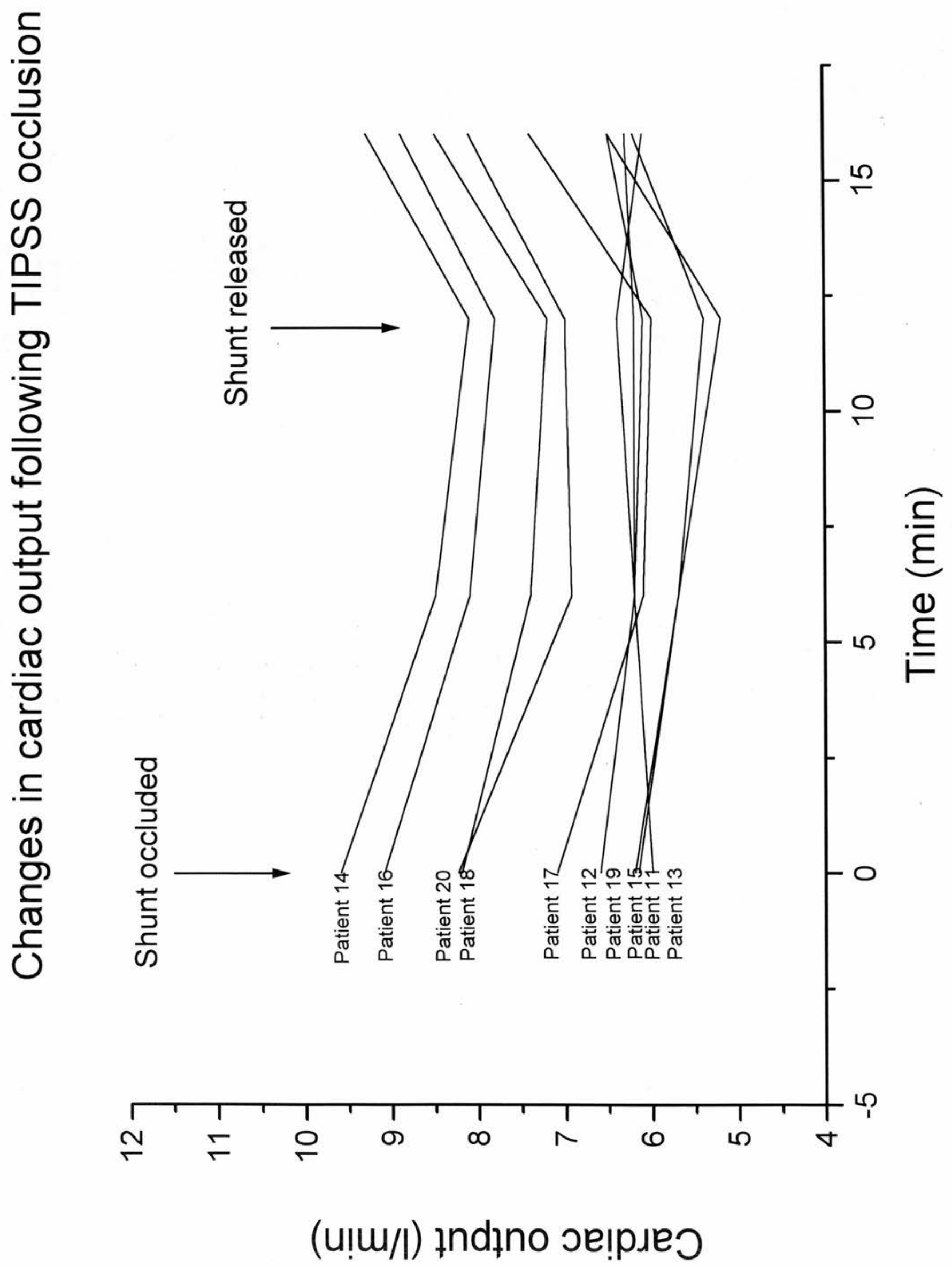




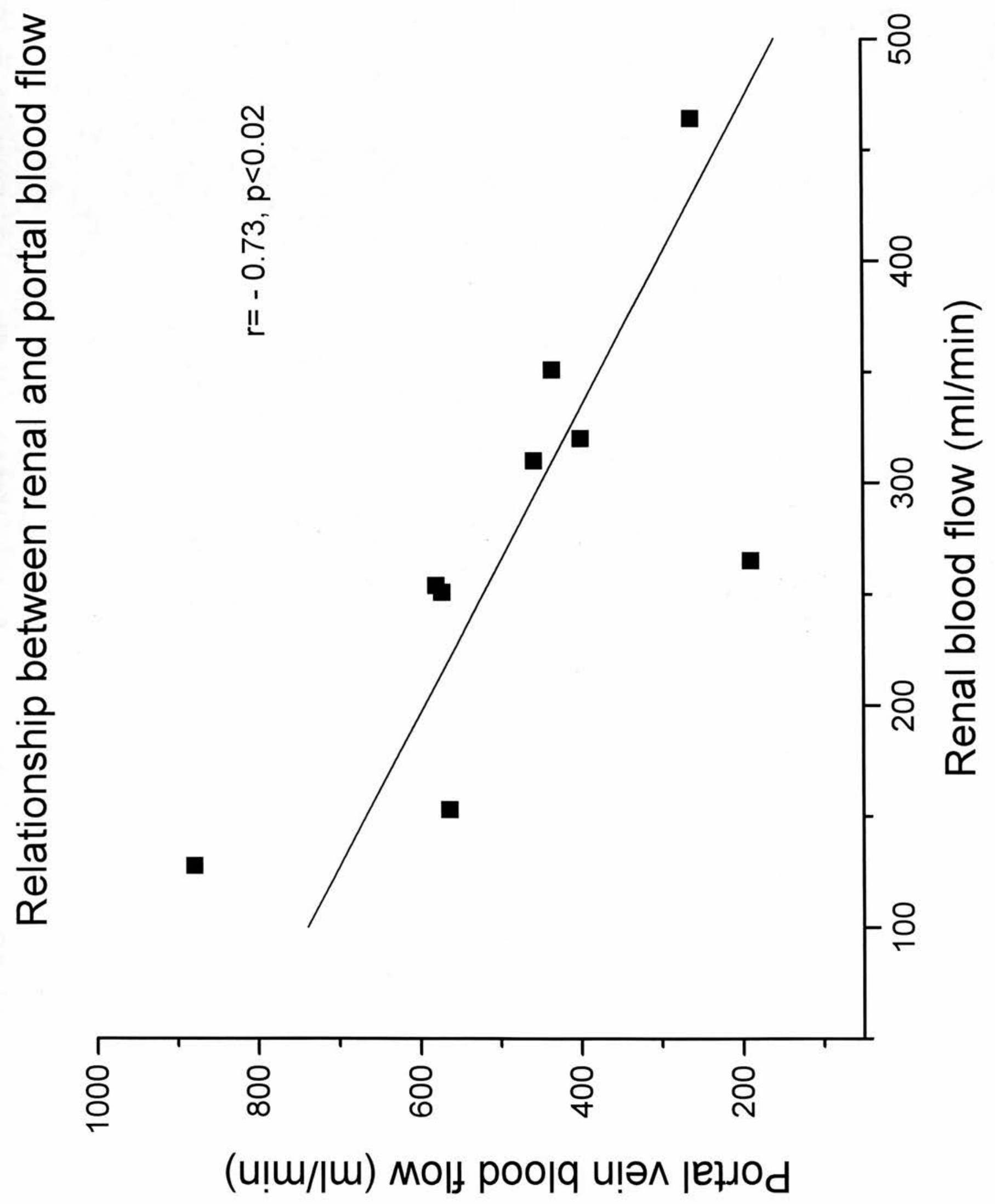




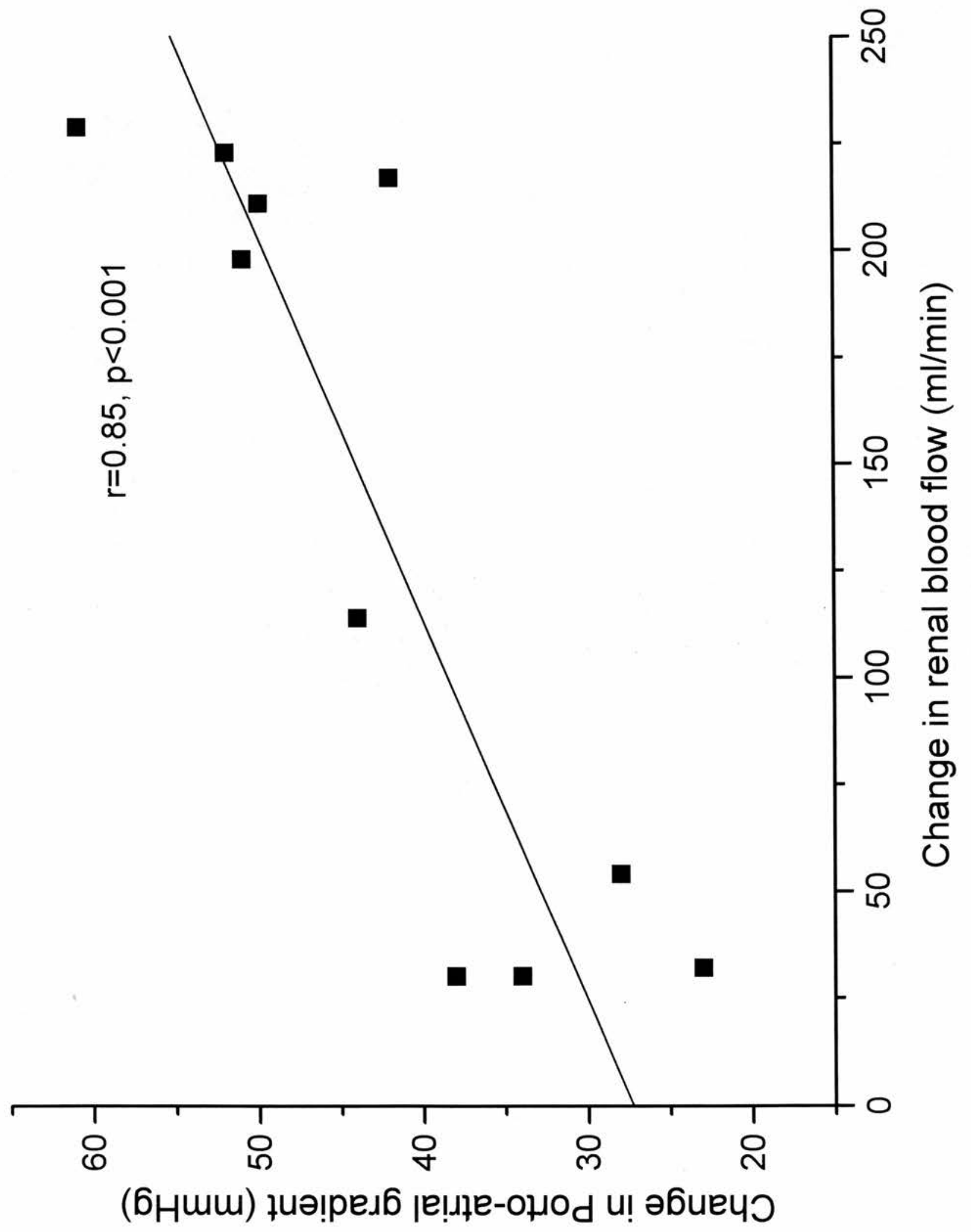




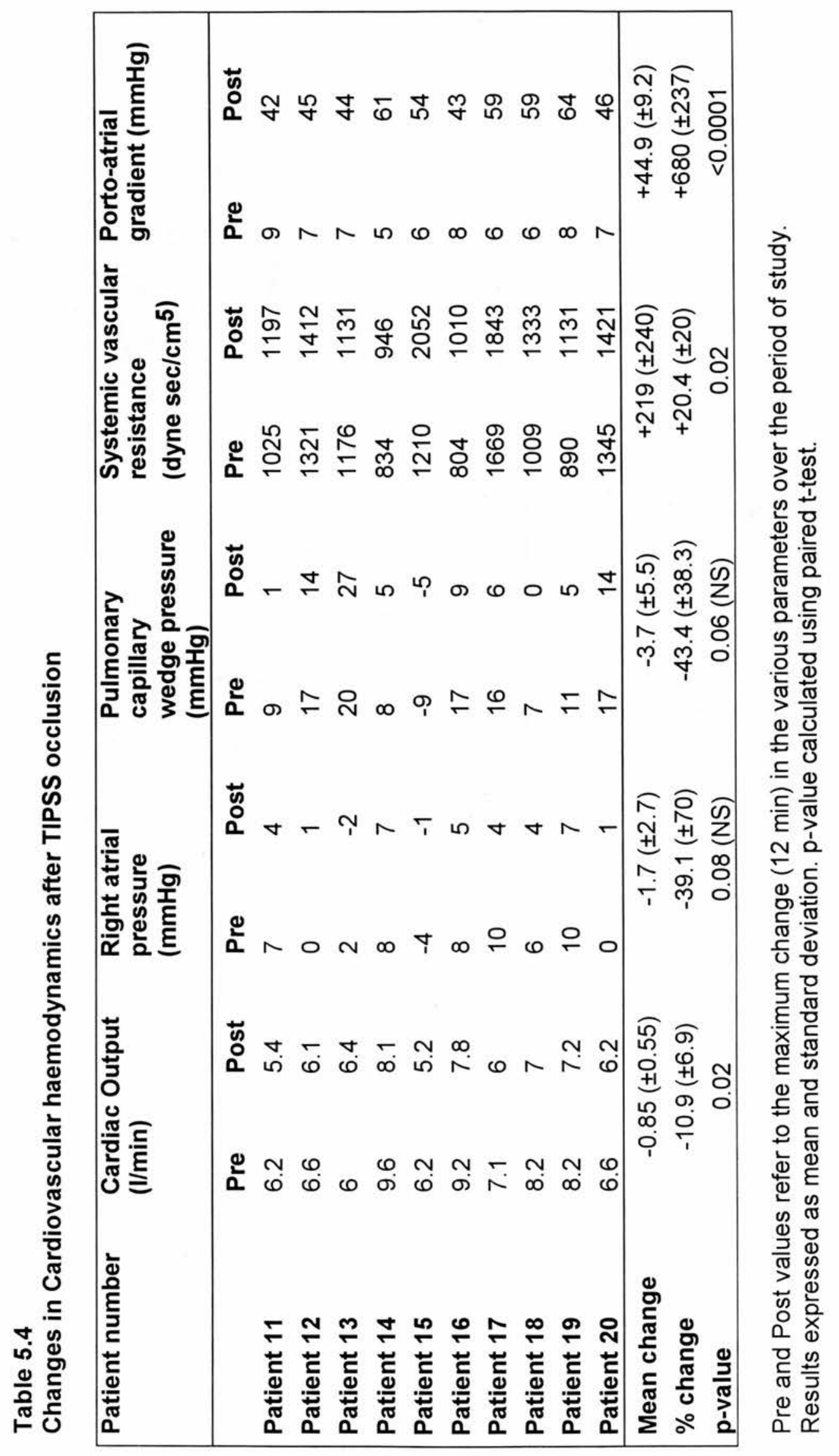




\subsection{Changes In The Neurohumoral Profile}

Blood for the measurement of neurohumoral factors was obtained from patients in group I. Concentrations of the different factors in the renal and systemic circulation prior to and following shunt occlusion are summarised in Table 5.5. Systemic concentrations of PRA, All, and cGMP were significantly higher in the patients compared to normal controls $(p<0.04, p<0.02$ and $p<0.01$ respectively). There was no significant difference in the adrenaline, noradrenaline, and ANP concentrations in the patients compared with age and sex matched healthy volunteers.

Concentrations of CGMP and noradrenaline were significantly higher in the renal compared with the systemic circulation $(p<0.01$ and $p<0.02$ respectively). PRA was significantly lower $(p<0.04)$ and there was no significant difference in the concentrations of All, ANP and adrenaline in the systemic and the renal circulations. Following shunt occlusion there was no significant change in the concentration of any of these variables in the systemic or the renal circulations. 


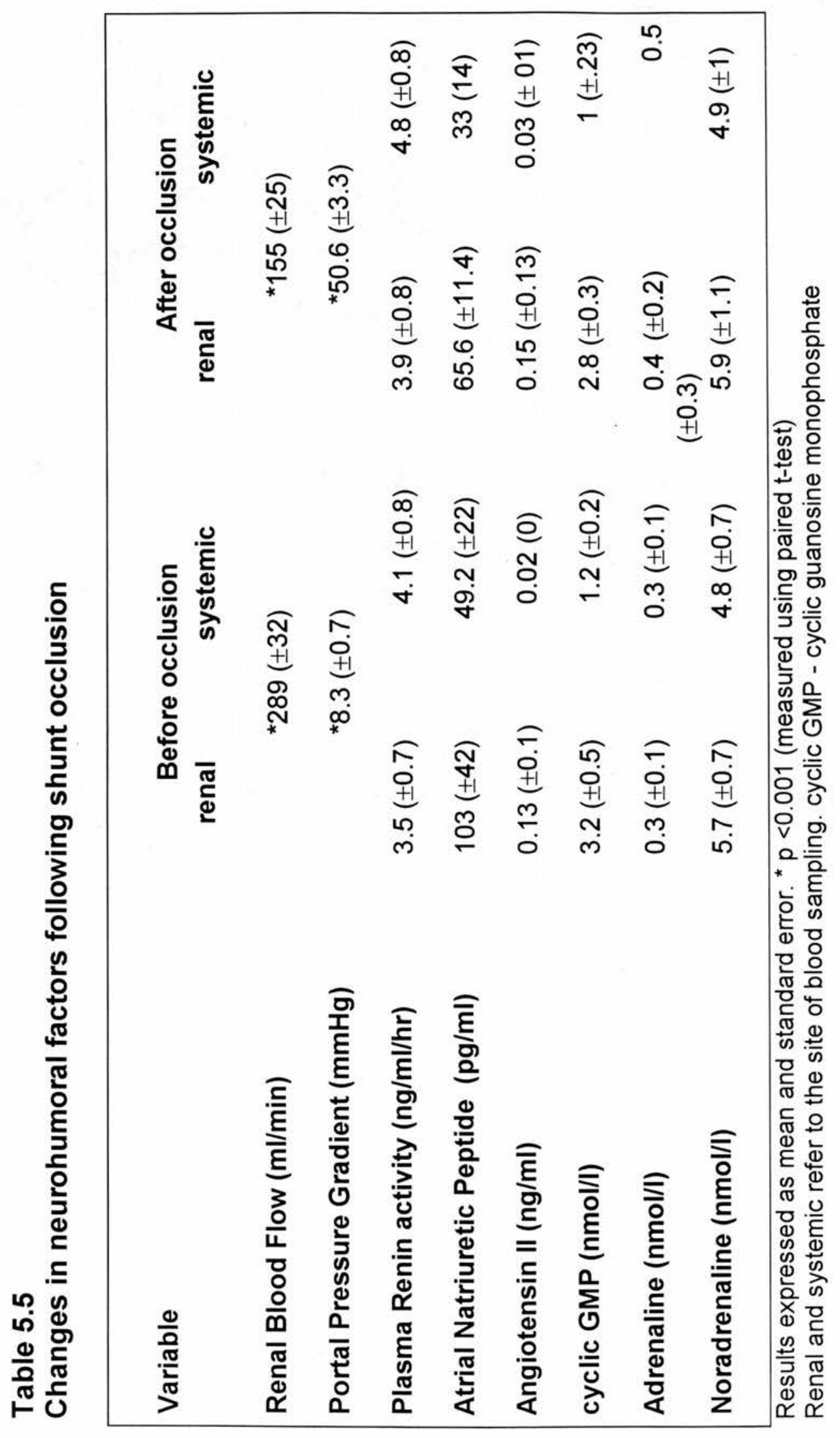




\subsection{SUMMARY}

The contribution of the reduction in cardiac output following shunt occlusion to the reduction in RBF cannot be disregarded. Occlusion of TIPSS appears to induce a decrease in venous return and therefore, possibly re-distribution of blood flow. However, the change in cardiac output did not produce any significant haemodynamic disturbance. The transient increase in RBF between 2 and 4 minutes in 3 patients following inflation of the balloon is difficult to explain but further argues against a significant role of reduction in $\mathrm{CO}$ being important in the change in RBF. Unfortunately, in this study we have not measured changes in $\mathrm{CO}$ at the same time points as measuring RBF. The statistical insignificance of the change in mean arterial pressure is unlikely to be due to the number of patients studied in each group because the difference remained inconsequential even when changes in both groups were considered together. Finally, as pointed out above, the degree of reduction in $\mathrm{CO}$ cannot fully explain the changes in $\mathrm{RBF}$.

In conclusion, we have demonstrated a dramatic reduction in RBF with acute increase in PAG which is compatible with the existence of hepatorenal reflex in man, although other mechanisms may also be contributory. This is a reproducible model which can be used to study this phenomenon further, enhance our understanding of the changes in renal circulation in liver disease thereby, providing clues for the development of newer treatments. 


\section{CHAPTER 6}

\section{CONCLUSIONS AND FUTURE}

PERSPECTIVES 
Transjugular intrahepatic portosystemic stent-shunt (TIPSS) has revolutionised the management of cirrhotic patients with variceal haemorrhage. This section deals with the discussion of the results of the various studies presented in this thesis in light of the available literature. The following discussion will highlight some of the important areas that require further evaluation

1. TIPSS procedure and shunt insufficiency

2. Prognosis of patients with TIPSS and predictive models

3. Hepatic encephalopathy

4. Variceal bleeding

5. Sodium handling after TIPSS

6. Hepatorenal reflex

\subsection{TIPSS PROCEDURE AND SHUNT INSUFFICIENCY}

Transjugular intrahepatic portosystemic stent-shunt (TIPSS) has revolutionised the management of cirrhotic patients with variceal haemorrhage (Rossle et.al. 1994). The occurrence of shunt insufficiency has led to considerable speculation about the long-term benefit of TIPSS but what is surprising is that this entity of shunt insufficiency remains poorly defined. Detection and treatment of shunt insufficiency is important to prevent variceal rebleeding. Numerous studies have addressed the rates of variceal rebleeding and there is remarkable consistency in the data which place the rate between 10 and 25\% (LaBerge et.al. 1993, Rossle et.al. 1994, Jalan 
et.al. 1994, Martin et.al. 1993, Perarnau et.al. 1993, Lind et.al. 1994, Stanley et.al. 1996). This could be prevented if shunt insufficiency could be identified and corrected before the rebleed. However, in order to estimate the prevalence and devise strategies to prevent and correct shunt insufficiency, it is important to define what is meant by shunt insufficiency.

The incidence of shunt insufficiency in published series varies between 25\% and 80\% (LaBerge et.al. 1993, Rossle et.al. 1994, Jalan et.al. 1994, Martin et.al. 1993, Perarnau et.al. 1993, Lind et.al. 1994, Stanley et.al. 1996) and is dependent upon the frequency of assessment of shunt function, the duration of follow up and the method of surveillance used. This remarkable variation in the rate of shunt insufficiency in the face of virtually constant reported incidence of variceal rebleeding is noteworthy and deserving of closer attention.

\subsection{Definition of shunt insufficiency}

The aim of TIPSS is to control variceal bleeding or prevent rebleeding by reducing the portal pressure gradient $(P P G=$ portal pressure - inferior vena cava pressure) to $<12 \mathrm{mmHg}$. This is considered by most authorities to be the critical figure below which variceal haemorrhage does not occur (GarciaTsao et.al. 1985). It is therefore reasonable to suggest that shunt insufficiency should be defined as an increase in the PPG to $>12 \mathrm{mmHg}$ in patients in whom the PPG was greater then $12 \mathrm{mmHg}$ at the time of insertion of TIPSS. 
However, about $15 \%$ of patients bleed from oesophageal varices with a PPG of less than $12 \mathrm{mmHg}$ (Jalan 1995). Furthermore, TIPSS is often inserted for the treatment of ascites (Ochs et.al. 1995) where the PPG at the time of insertion may be less than $12 \mathrm{mmHg}$. These issues complicate the definition of shunt insufficiency. The definition of shunt insufficiency must therefore take into account the PPG at the time of insertion of TIPSS. Any degree of shunt narrowing that is not associated with significant haemodynamic phenomenon should not be considered significant. The precise definition of shunt insufficiency in these patients in whom the PPG is $<12 \mathrm{mmHg}$ at the time of insertion of TIPSS is currently being debated. In this population of patients we define shunt insufficiency as an increase in PPG of $20 \%$ or more above the value obtained immediately after TIPSS (Jalan et.al. 1994, Stanley et.al. 1996).

Similarly, in patients with variceal haemorrhage and a PPG of $>12 \mathrm{mmHg}$, if the placement of TIPSS does not reduce the PPG below $12 \mathrm{mmHg}$ or, in patients with a PPG of $<12 \mathrm{mmHg}$ at the time of insertion of TIPSS the pressure is not reduced by $20 \%$ or more, then it should be considered inadequate.

\subsection{Pathogenesis of shunt insufficiency}

What is apparent from the literature is that shunt insufficiency occurs in two distinct forms (LaBerge et.al. 1993, Jalan et.al. 1994). The first is shunt thrombosis which is associated with dramatic increases in the PPG and occurs very early after the insertion of the shunt. Its true incidence is 
impossible to ascertain because routine portography is not often performed early enough after the insertion of TIPSS but studies which have performed routine portogram at 1 month suggest that its incidence is about $15 \%$. The second type is pseudo-intimal hyperplasia, which occurs in the intraparenchymal portion of the shunt or in the hepatic vein above the stent. This is noticeable about 1 month or more following insertion of TIPSS, is slowly progressive and probably affects all the shunts to a variable extent. The reported incidence of this type of insufficiency is widely variable and depends almost entirely upon the duration of follow up and the frequency of the surveillance.

Why shunt insufficiency occurs is unclear and the pathogenesis of the two types are probably different. Unlike an intravascular shunt, TIPSS encounters the liver parenchyma and its secretions. More than one group have independently suggested that seepage of bile due to transection of a major bile duct plays an important role in the development of shunt thrombosis (LaBerge et.al. 1993, Jalan et.al. 1994). The later shunt dysfunction is associated with pseudo-intimal hyperplasia and it appears that its pathogenesis is multifactorial and includes contributions from bile, the effect of turbulence, factors related to the radiological technique, stent characteristics, patient selection and some hitherto undefined factors related to the repair process (LaBerge et.al. 1993, Jalan et.al. 1994).

\subsection{Shunt surveillance}


The most important questions that relate to everyday practice and need answering are:

(i) what is the effect of shunt insufficiency and is shunt surveillance necessary?

(ii) If necessary, when and how shunt surveillance should be performed and

(iii) if shunt insufficiency is detected what should be done?

It appears that the introduction of TIPSS alters the natural history of variceal haemorrhage such that rebleeding is not the consequence in every patient with an insufficient shunt but tends to occur in about $30-50 \%$ (LaBerge et.al. 1993, Rossle et.al. 1994, Jalan et.al. 1994, Martin et.al. 1993, Perarnau et.al. 1993, Lind et.al. 1994, Stanley et.al. 1996) patients in whom the shunt function is compromised. However, variceal rebleeding after insertion of TIPSS occurs almost exclusively in patients with shunt insufficiency (LaBerge et.al. 1993, Rossle et.al. 1994, Jalan et.al. 1994, Martin et.al. 1993, Perarnau et.al. 1993, Lind et.al. 1994, Stanley et.al. 1996) and therefore surveillance is necessary to prevent rebleeding.

A pointer as to when shunt function should be assessed is provided by the observation that the rate of rebleeding is split almost equally between a few days of the TIPSS and the remainder of follow up (LaBerge et.al. 1993, Rossle et.al. 1994, Jalan et.al. 1994, Martin et.al. 1993, Perarnau et.al. 1993, Lind et.al. 1994, Stanley et.al. 1996) (Figure 6.1). It would therefore seem logical that the rates of rebleeding could be halved if the episodes of shunt insufficiency that result from thrombosis can be identified early and corrected. Shunt function should therefore be assessed early, i.e. within a week of 
TIPSS to detect early shunt thrombosis and then follow up should probably be performed at 3 months and then 6 month intervals to identify shunt insufficiency secondary to pseudo-intimal hyperplasia.

How they should be identified has been the subject of a number of investigations. The two methods in current usage are Doppler ultrasonography and portography with measurement of portal haemodynamics. Although some investigators have found Doppler ultrasonography with measurement of flow velocity in the portal vein to have a close correlation with shunt function (Jalan et.al. 1994, Dabos et.al. 1996), our prospective comparison of Doppler ultrasonography with portography and measurement of portal pressure was disappointing and suggested that portography should still be considered the 'gold standard' (Jalan et.al. 1994). These differences may stem from operator characteristics or may be related to the fact that assessments of shunt function in our studies were performed on a routine ultrasonography list. A reasonable compromise may be to combine the two procedures judiciously considering the invasive nature of portography. One approach may be to use Doppler ultrasonography as a screening method, maintaining a low threshold to proceed to portographic assessment. Another approach may be to use changes in flow characteristics of the portal vein haemodynamics following TIPSS to act as a guide to detect shunt insufficiency.

Finally, once shunt insufficiency is identified what should we do about it? The approach to this problem is based upon the proposed pathogenic mechanisms of shunt insufficiency and long term follow up of patients with 
TIPSS. Early shunt insufficiency is almost entirely due to shunt thrombosis which occurs in association with transection of major bile ducts. Balloon angioplasty is invariably followed by re-occlusion. These patients should have parallel shunts. Patients with pseudo-intimal hyperplasia either in the hepatic vein or in the intraparenchymal portion of the shunt usually respond and maintain shunt patency with balloon angioplasty alone (Jalan et.al. 1995, Dabos et.al. 1996). The problem that is beginning to emerge with increasing experience and longer follow up is that of the occlusion of the second shunt. Whether a third shunt should be considered, whether they should undergo repeated balloon angioplasty, whether they should have shunt surgery or whether we should revert to endoscopic treatment of varices remains unanswered.

The available data would suggest that a single early assessment of shunt function and correction of the underlying insufficiency would halve the rebleeding rate after TIPSS. The final impact of TIPSS in the management of variceal haemorrhage will become apparent only when the problem of shunt insufficiency has been solved. Future research should be directed at noninvasive methods of prediction of shunt insufficiency and the development of stents with intraparenchymal covering.

\subsection{MORTALITY}

When TIPSS is inserted for variceal haemorrhage, there is transient worsening of liver function in approximately one third of the patients and this 
may precipitate liver failure the severely ill patients (Jalan et.al. 1997, Sanyal et.al. 1996). Thirty day mortality after TIPSS is about $20 \%$ and one year survival rates vary from 50-85 (LaBerge et.al. 1993, Rossle et.al. 1994, Jalan et.al. 1994, Martin et.al. 1993, Perarnau et.al. 1993, Lind et.al. 1994, Stanley et.al. 1996). Mortality after TIPSS seems to be related to the severity of the underlying liver disease (Jalan et.al. 1995, Somberg et.al. 1992). Accurate identification of patients likely to die early after TIPSS would help in the selection of patients for the procedure and plan on early orthotopic liver transplantation.

Prognosis is conventionally assessed using Cox's proportional hazard regression analyses (Cox DR, 1972). In patients with active variceal haemorrhage treated with sclerotherapy, prothrombin time, encephalopathy, severity of liver disease (LeMoine et.al 1992) and the amount of blood transfusion in the first 72 hours have all been suggested as independent predictors of early mortality (Jacob et.al. 1989). Similar observation of high rates of early mortality for patients with worse Child class is evident in surgical series (Planas et.al. 1992).

Although the Cox models have been assumed to have sufficient precision to estimate outcome there are some problems with its usage in predicting individual prognosis. First, the prognostic index reflects only average prognosis with wide variation in individual prognosis. Previous studies assessing outcome in patients with primary biliary cirrhosis (Christensen et.al. 1985) have confirmed a marked difference between observed and predicted survival. This imprecision may occur due to their 
inability to evaluate variables with sufficient prognostic information. Second, these statistical methods are limited by their inability to assess non-linear relationships between variables. Third, the Cox models have very limited capacity for learning. This limits its ability to deal with factors that change suddenly, especially in patients with variceal bleeding such as sepsis and rebleeding.

The present predictive models of early mortality after TIPSS are based upon retrospective data and suffer from poor specificity. Severity of liver disease as judged by Child class (Rubin et.al. 1995), APACHE II (Rubin et.al. 1995), a combination of Child class and haemodynamic instability (Helton et.al. 1993), a combination of creatinine, bilirubin, prothrombin time and aetiology of liver disease (Malinchoc et.al. 1996), a combination of Child class and hyponatraemia (Jalan et.al. 1995) have all been suggested as predictors of early mortality after the insertion of TIPSS. However, these data have not been tested prospectively and have poor sensitivity and specificity. The reason for this poor specificity may relate to heterogeneity of the data used to develop the regression models, or may reflect the limitation of linear models as described above. Somberg et.al. (1992) have suggested that aspartate aminotransferase, bilirubin and Child class were independent predictors of mortality. Gordon et.al. developed a prognostic model using bilirubin and creatinine to divide patients into a high and low risk categories. The lack of prospective, blinded evaluation of the models makes these difficult to use in a clinical situation. The large number of variables identified in the different studies as predicting early mortality suggests that it is unlikely 
that one or two variables will determine final outcome accurately. This is the major attraction of a model based upon neural networks because it has the ability to simultaneously look at all the variables together.

We have recently used a 'feed forward' kind of neural network which implies that signals flow only in one direction, i.e. from input to output thereby, resembling the regression procedure. The learning of a 'feed forward' network is associative where, the network learns to associate one type of data with another. In an on-going study, an artificial neural network has been trained to predict early mortality using data from a hundred patients from two centres. Early results (presented to AASLD, 1997, Jalan et.al. 1997) showed that the network predicted the outcome correctly in 186 patients (93.5\%). The sensitivity of accurate prediction of early mortality was $67 \%$ and the specificity was $94 \%$ with a positive predictive value of $91 \%$. The five most weighted variables that predicted early mortality were the prothrombin time, serum creatinine, need for mechanical ventilation, bleeding from gastric varices and urgent TIPSS for uncontrolled variceal bleed. The development and training of a neural network does not differ from any other data derived decision making aid or from statistical analysis but it appears that a significantly smaller sample size is required for determining outcome accurately. Further datasets would be needed to develop this model further.

\subsection{ENCEPHALOPATHY AND LIVER FUNCTION}


The controlled evaluation of neuropsychological function confirms that subclinical encephalopathy occurs in about $67 \%$ patients with cirrhosis. The major criticism that has been levied against the use of the computerised neuropsychological assessment is that although they are scientifically valid and reliable, these assessments do not accurately reflect the "real life" functioning capability of the patient. The addition of Rivermead Behavioural Memory Test, which is a measure of everyday memory difficulty serves to address this criticism. Furthermore, with one exception, subclinical encephalopathy did not progress to clinical encephalopathy in this study. It is interesting that one patient did however, become profoundly encephalopathic which was refractory to all forms of medical and radiological intervention. We would like to re-emphasise that the group of patients we were studying were relatively well and had no evidence of clinical encephalopathy prior to the study.

There was significant deterioration in both the simple and complex reaction time sub-tests of CANTAB following TIPSS at 3 and 9 months suggesting a worsening in neuropsychological function following TIPSS. Similar deterioration was noted in the cirrhotic controls suggesting that this change may be due to the progression of liver disease rather than the effect of TIPSS per se. Longer follow up of changes in neuropsychological function in cirrhotic controls would have been interesting. Significant improvement in RBMT at 1 month and of the delayed matching to sample memory sub-test of CANTAB at 15 months is somewhat surprising and suggests that the mechanism of changes in the memory characteristics in patients with sub 
clinical hepatic encephalopathy is different to the pathogenic mechanisms associated with the changes in the neuropsychological function.

Patients in the TIPSS group were significantly less anxious at 1 month but by 3 months became more anxious. This may be related to the security of the in-hospital stay, regular follow up, familiarity with the medical staff and ready access to the hospital services. Subsequent deterioration may be due to the effect of return to the community. Significant improvement in the levels of anxiety in the control population at 1 month may be related to the familiarity with the operators.

The psychological component of the quality of life deteriorated significantly in patients in the TIPSS group, starting at 3 months after the procedure and continuing up to the duration of follow up. This may be a surrogate marker of anxiety. No significant change in the physical quality of life argues against the change in the psychological component being due to a worsening in the severity of liver disease. Whether this finding is the effect of TIPSS is difficult to ascertain because of the shorter duration of follow up in the cirrhotic controls. It is possible that the mechanisms associated with changes in the neuropsychological function (reflected by changes in CANTAB) and psychological component of the quality of life are similar.

Of particular interest is the finding of a significant correlation between changes in ICG clearance and change in the complex and simple reaction time sub tests of CANTAB. This observation is in keeping with the study by Lacy et. al. (1992) who demonstrated a similar phenomenon in patients having distal splenorenal shunts. It is possible that some of the alteration in 
ICG clearance may reflect changes in liver function following TIPSS and some of the alteration may be due to changes in portal blood flow due to placement of the shunt.

Somberg et.al. (1992) suggested that the development of encephalopathy following TIPSS was predicted by the aetiology of the underlying liver disease (non alcoholic), female sex and low serum albumin. Our evaluation of the predictors of encephalopathy have failed to confirm this observation and found that encephalopathy following TIPSS is independently predicted only by its presence prior to TIPSS (Jalan et.al. 1995). Sanyal et.al. (1994) have confirmed this finding in an independent study.

Significant deterioration in the markers of hepatocellular injury (ALT) and function (bilirubin) following TIPSS is not unexpected. Simultaneous reduction in ICG clearance suggests that this may be the result of the phenomenon of "sinusoidal steal" in addition to dysfunction of the liver. A recent abstract suggested that this deterioration in liver function, particularly in the activity of the microsomal system (P450) may be more prolonged than that observed in this study (Lotterer et.al. 1994). In extreme cases patients after TIPSS may develop a fulminant failure like syndrome which is invariably fatal (Jalan et.al. 1997).

Our results and the incidence of clinically detectable encephalopathy following TIPSS is at variance with that reported in a study comparing the development of encephalopathy in patients having TIPSS and those being treated by sclerotherapy which found that encephalopathy following TIPSS occurred in about $30 \%$ patients (Sanyal et.al. 1994). The population studied 
comprised all comers with a large proportion belonging to Child class $\mathrm{C}$ whereas we have selected the study population excluding patients who were clinically encephalopathic, in renal failure, had continued bleeding or were septic. In addition only a small proportion of our patients suffered from severe liver disease. The purpose of our study was to assess the contribution of TIPSS to the alteration of neuropsychological function, memory and quality of life in patients who were not clinically encephalopathic.

In summary, clinical encephalopathy in this selected group of patients occurred in $3 \%$ patients following TIPSS. Neuropsychological tests were significantly worse in cirrhotic patients compared with controls, $67 \%$ showing evidence of subclinical encephalopathy. In this selected group of patients with no history of encephalopathy, there was a temporary deterioration in the neuropsychological function both in patients having TIPSS and the cirrhotic controls. Although temporary deterioration occurred in the levels of anxiety and the psychological component of the quality of life in patients with TIPSS, measures of memory improved. Significant deterioration occurred in ALT, bilirubin and ICG clearance. Changes in ICG clearance following TIPSS correlated significantly with the changes in the neuropsychological tests. Further studies should address the mechanism of these changes and the effect of different prophylactic measures on their prevention. Repeated access to the portal system provides a unique opportunity to determine the role of gut derived factors in the pathogenesis of HE that follows TIPSS.

6.4 VARICEAL HAEMORRHAGE 
Secondary prophylaxis

The Royal Infirmary of Edinburgh is a tertiary referral centre for the treatment of liver diseases and it is also the only liver transplantation centre in Scotland. Consequently, we are referred patients from the whole of Scotland who are often treatment failures. The purpose of this study was to look at the role of TIPSS in the secondary prophylaxis of variceal haemorrhage in patients with cirrhosis. Previous studies have suggested that the natural history of variceal hemorrhage is extremely variable depending upon when patients are recruited into the studies and how many bleeds they have had at the time of randomisation (Smith $\mathrm{JL}$ and Graham DY, 1981). Therefore, in this study we randomised the patients who presented with the first episode of variceal haemorrhage and were not bleeding at the time of randomisation. Therefore the 12 patients who rebled within 24 hours of the initial endoscopy were excluded from the study.

Our randomised trial has shown that TIPSS markedly reduces the frequency and severity of rebleeding in patients presenting with a first episode of variceal haemorrhage compared with VBL. There are two major factors which affect rebleeding in patients with cirrhosis after the first episode of bleeding has been controlled. First, the risk of rebleeding and death is highest in the first week after the initial haemorrhage and decreases thereafter (Smith JL and Graham DY 1981, Burroughs et.al. 1992). Second, the rates of rebleeding within 2 months in different series depends almost entirely upon the number of patients with advanced liver disease included 
into the study (Smith JL and Graham DY 1981, Burroughs et.al. 1992, Lebrec et.al. 1984, Burroughs et.al. 1983, Villeneuve et.al. 1986, Quieniet et.al. 1987, Colombo et.al. 1989, Garden et.al. 1990). These two factors are crucial when assessing the impact of a new modality of treatment for variceal bleeding.

There are four fully published randomised controlled clinical trials comparing TIPSS with endoscopic sclerotherapy (ES) in the management of variceal hemorrhage (Cabrera et.al. 1996, Rossle et.al. 1997, Sanyal et.al. 1997, Cello et.al. 1997). Interpretation of trials in variceal hemorrhage have inherent difficulties because of differences in the management of patients in different parts of the world. TIPSS and endoscopic treatment can be difficult to compare, for whereas the success of a TIPSS procedure is apparent immediately from a reduction in portal pressure to less than $12 \mathrm{mmHg}$, there is no immediate indicator of a successful endoscopic sclerotherapy treatment. Furthermore, separation of patients in these trials into those who are treated by endoscopic therapy and those who had TIPSS is less clear. Endoscopic therapy was used to treat the index bleed in both groups of patients. Therefore, most patients in the TIPSS group will have had at least one session of endoscopic treatment. Cross-over between groups because of treatment failures also complicate the 'intention to treat' analysis.

All of these trials have built a potential confounding factor into the study design which, although ethically responsible, makes interpretation of the mortality data difficult. Rates of rebleeding were significantly lower for TIPSS than for ES in Cabrera's $(p<0.02)$, Rossle's $(p=0.001)$ and also in 
Cello's study $(p<0.01)$. No significant differences in rebleeding were detected in Sanyal's study. In Cabrera's study, 51\% patients (16 patients) rebled in the ES group. Of these, 10 patients had uncontrolled variceal hemorrhage and 9 were 'rescued' with TIPSS. In Rossle's study, 45\% (29 patients) patients rebled in the ES group and 9 patients underwent 'TIPSS rescue'. In Cello's study, $48 \%$ patients (12 patients) rebled in the ES group of whom 6 patients required 'TIPSS rescue'. In Sanyal's study only $26 \%$ (8 patients) patients rebled in the ES group, but 6 of them required TIPSS rescue. Although, it is considered 'valid' to analyse data on an intention to treat basis this does not reflect the true result. Mortality from uncontrolled variceal hemorrhage in patients with cirrhosis who are treated with conventional measures approaches $80 \%$. The use of TIPSS in this situation improves survival (Jalan et.al. 1995). If these studies were not designed to rescue patients in the ES arm, each of these studies might have shown a survival benefit for the patients in the TIPSS group. In fact, Garcia-Villareal (1996) presented a similar randomised trial in an abstract form in which 'TIPSS rescue' was not used. Although they randomised only a small number of patients they showed a significant survival benefit for patients randomised to TIPSS. This tends to support the suggestion that the lack of significant differences in mortality in the first 3 studies may be due to rescue TIPSS intervention for uncontrolled variceal haemorrhage. It could therefore be argued that these trials are not really TIPSS versus ES but TIPSS versus ES plus rescue TIPSS trials. 
Of the above trials, the study by Sanyal et.al. stands out in not reporting a difference in rebleeding. This is probably because of the study design and the methodology used in this study. One of the most important factors that determines outcome in clinical trials of variceal bleeding is the time interval from the episode of the first bleed to randomisation. Graham and Smith (1981), and Burroughs et.al. (1989) have confirmed in separate studies that shifting the starting point for analysis (time of randomisation) of survival and rebleeding from the episode of bleeding by as little as 5 days significantly alters the rates of rebleeding and mortality. They also showed that if treatments to prevent rebleeding were used long after control of a first episode of bleeding then improved survival would not be expected as many deaths related to bleeding would already have occurred and the risk of death in the survivors is much lower after this period. The time of randomisation in the first 3 trials was within 3 days of the bleed (Rossle - within 24 hours of presentation, Cabrera - within 3 days after control of bleeding, Cello - within 24 hours of admission). In Sanyal's study the exact time of randomisation is not clear. They state that 'survivors of an episode of active esophageal variceal hemorrhage were considered for inclusion if they were clinically stable and were not actively haemorrhaging (absence of hemorrhage was indicated by a stable hemoglobin level and no need for transfusions) for at least 72 hours.' They then state that that 'the primary intervention was started within 72 hours of randomisation.' This suggests that randomisation was performed within 3 days of the initial hemorrhage but in 'Table 1' they describe that $9 \pm 2$ days elapsed between the time of cessation of bleeding 
and TIPSS insertion, and $11 \pm 3$ days elapsed between the time of cessation of bleeding and endoscopic sclerotherapy. What is also unclear is that if the criteria for randomisation were met at 72 hours then why did patients receive their respective treatments at a mean of 9 and 11 days respectively. The rebleeding rate in their sclerotherapy group is only $26 \%$ which is lower than that reported in other studies above. This may have been due to a selection bias whereby they have inadvertently selected survivors likely to do well with sclerotherapy.

No significant differences were observed in mortality in the first 3 studies, but an essential confounding factor is the 'TIPSS rescue'. Sanyal's study however, did show a significantly higher rate of mortality in the TIPSS group. When a proportional hazards model was constructed using variables such as age, Child-class, encephalopathy, alcohol use and the primary intervention (TIPSS or ES), none of these factors were found to be independent predictors of mortality. It is also interesting to look at the causes of mortality. In the TIPSS group, of the 12 deaths, 5 were due to variceal rebleeding. Seven patients died in the ES group. Only 3 deaths were due to variceal rebleeding. Variceal rebleeding in the TIPSS group accounted for zero deaths in Cabrera's study and zero deaths in Rossle's study. Data for Cello's study are not available. Three deaths occurred due to rebleeding in the ES group in Cabrera's study and 2 deaths were related to variceal rebleeding in Rossle's study. The possible reason for the higher rate of variceal hemorrhage in the TIPSS group in Sanyal's study may be related to the TIPSS procedure itself. TIPSS was either unsuccessful or inadequate in 
5 patients (12\%). Furthermore, most authorities would agree that the portal pressure gradient (PPG) should be reduced to less than $12 \mathrm{mmHg}$ after TIPSS. If this is not achieved then the procedure should be considered unsuccessful. Although Sanyal et.al. state that some of their patients had a PPG of $>12 \mathrm{mmHg}$ they do not say how many. They did not use a parallel stent to try and achieve this aim. The mean portal pressure they recorded at 6 months was about $18 \mathrm{mmHg}$ suggesting that their method of surveillance, i.e. endoscopy and Doppler ultrasonography did not adequately detect an increase in PPG. These factors may have contributed to the higher rate of rebleeding and mortality they observed in the TIPSS group.

There is consistency in 3 of the studies about the incidence of encephalopathy amongst the two groups of patients. Worsening in or the occurrence of previously absent encephalopathy was significantly higher and occurred in about $30 \%$ patients in the TIPSS group (Cabrera et.al. $33 \%$, Rossle et.al. 36\%, Sanyal et.al. 31\%) and about $15 \%$ in the sclerotherapy group (Cabrera et.al. 13\%, Rossle et.al. 18\%, Sanyal et.al. 13\%). Encephalopathy in Cello's study was not significantly different in the two groups (TIPSS - 50\%, ES - 44\%). This observation is at variance with the other studies and may be due to the patients studied and the method of diagnosis of encephalopathy. In this study, only patients classified as having either massive or sub-massive hemorrhage were included into the study. The authors do not mention any strict criteria for the diagnosis of encephalopathy which is defined in this study as 'the presence of asterixis, gross disorientation or agitation, or frank somnolence or coma in the absence 
of any other identifiable cause.' This definition would identify only patients with severe encephalopathy and overlook patients with milder encephalopathy. Only in Rossle's study are clear diagnostic criteria set out for grading the severity of encephalopathy. It is notable that only a handful of patients in this study did not respond to medical treatment (3\%) but responded quickly to reduction of the shunt. What is even more interesting is that there were 2 patients in the ES arm who developed encephalopathy that was refractory to treatment. These two patients had undergone 'TIPSS rescue'. These observations are important because they highlight that the introduction of TIPSS is associated with increased incidence of encephalopathy which is seldom troublesome, but also illustrate the shortcoming of an intention to treat analysis.

Cello's paper provides the first evaluation of the relative costs incurred in the management of patients with variceal hemorrhage using TIPSS or ES. They found no significant differences in either the total health care costs or in the mean health care cost per day of survival. There were no significant differences in the mean duration of hospitalisation amongst the groups for encephalopathy. Sanyal et.al provide some data regarding the reasons for hospitalisation which was greater for the patients in the ES group for decompensated ascites, and greater in TIPSS group for encephalopathy and sepsis. Rossle et.al. found that patients in the TIPSS group spent less time in the hospital, but this was not statistically significant. Cabrera et.al. found that although there was no significant difference in the number of days spent in the hospital by patients in either group for the index admission, patients in the 
ES group spent significantly more time in the hospital for rebleeding. Overall, the total number of days spent per patient in the hospital was roughly 27 days in the ES group and 25 days in the TIPSS group (mean from Cabrera's, Cello's and Rossle's study, data from Sanyal's study was not available). It would have been interesting to see the extent to which patients were readmitted to intensive care, high dependency care or ward care as these are important variables that make enormous differences to the overall cost. We compared TIPSS with variceal band ligation and found that patients in the banding arm required significantly more admissions for intensive and high dependency care (Jalan et.al. 1997). Indeed, overall costs/patient were significantly lower in the TIPSS group. We accept, however, that measuring costs of different procedures and extrapolating them to different health systems is fraught with inaccuracies. Despite the differences in the data it is clear that TIPSS is not more expensive than ES in the management of variceal hemorrhage in patients with cirrhosis.

Another confounding factor that makes interpretation difficult is that these studies do not strictly look at secondary prophylaxis of variceal hemorrhage, but at the role of the two treatments in the management of any episode of variceal bleeding. By definition, a study of secondary prophylaxis should include only patients who are presenting with a first episode of variceal hemorrhage, and build into the study an exclusion for patients who have bled previously. Patients with second or subsequent variceal bleeding are the survivors of a first bleed and may intrinsically have a different natural history. Cabrera randomised about $30 \%$ of patients in either group with 
previous variceal bleeding. In Rossle's study about $60 \%$ patients had previous bleeds and in Sanyal's study about $50 \%$ patients had previous bleeds. Cello et.al. do not mention previous bleeds as an exclusion criteria and do not state how many of the patients in either group had previous bleeds.

The fundamental principle of power calculations for clinical trials is to estimate the number of patients that would need to be randomised to show a difference in the primary end point of the study. The primary end-point of the studies by Cabrera et.al., Rossle et.al and Sanyal et.al. were rebleeding and the power calculations were made to this end. Cello et.al. state that the primary end point of their study was survival, but their power calculations for estimation of sample size were for rebleeding. If mortality is considered a secondary end point, as in all these studies, then it is inadvisable to overinterpret these data and say that 'no differences in mortality were detected'. A study specifically designed to answer this question needs to be performed before such a conclusion could be made.

One method for overcoming the problems of sample size is the performance of a meta-analysis, but this can only be reliable if the studies are homogenous. According to Friedman and Goldberg (1996), 'heterogeneity is the Achilles heel of a meta-analysis.' The above account plus other confounding factors such as the use of different treatments in different studies such as banding, propranolol, vasoactive drugs and methods for shunt surveillance must make the studies so heterogeneous that they will be difficult to combine. The interpretation of data from such an 
analysis due to the degree of heterogeneity will be extremely difficult. Regarding meta-analyses, Bailar (1997) concluded that 'in my own review of selected meta-analyses, problems were so frequent and so serious, including bias on the part of the meta-analyst, that it was difficult to trust the overall "best estimates" that the method often produces'.

These studies are, however, extremely valuable in their own right and show five important conclusions. There is little doubt that TIPSS substantially reduces rebleeding, its severity and hospital admissions from this complication. One of the major problems following TIPSS is shunt insufficiency which can be prevented and does not translate into increased rebleeding if shunt surveillance is performed regularly. TIPSS is associated with increased rates of encephalopathy which is seldom troublesome. There is no difference in survival between the two treatment modalities but this must take into account the effect of 'TIPSS rescue' in patients with uncontrolled variceal hemorrhage in the patients in the endoscopy arm. Finally, TIPSS certainly does not cost more to the health service but may in some centres be associated with reduced costs.

The rate of variceal rebleeding in our VBL group was higher than reported in studies comparing VBL with sclerotherapy. Most studies report rates of rebleeding following VBL of $25-35 \%$ (Steigmann et.al. 1992, Gimson et.al. 1993, Lo et.al. 1995, Hou et.al. 1995, Hashizume et.al. 1993, Laine et.al. 1996) whereas the rate of rebleeding in our VBL group was $51 \%$. This difference is most likely due to different populations under study. All the above studies randomised about $30 \%$ of patients with Child $\mathrm{C}$ liver disease 
and this is reflected by about $30 \%$ rebleeding in these studies (Steigmann et.al. 1992, Gimson et.al. 1993, Lo et.al. 1995, Hou et.al. 1995, Hashizume et.al. 1993, Laine et.al. 1996). About $50 \%$ of patients in our study had Child class C liver disease, and, as we have outlined earlier the risk of rebleeding is closely related to the severity of liver disease. The effect of severity of liver disease upon rebleeding is highlighted in this study as we have shown a significantly higher rate of rebleeding in patients with Child class $\mathrm{C}$ disease compared with patients with Child class $A$ and $B$ disease. Furthermore, most of our patients continued to abuse alcohol which is known to have a detrimental effect upon portal hemodynamics (Silva et.al. 1994). Although some authorities suggest that earlier eradication of varices would occur if more than 1 band were placed on an individual varix, this concept is not universally accepted. At the time this study was set up we decided to follow the protocol and the method that was described by Steigmann et.al (1992). The number of sessions of VBL required to achieve variceal eradication was similar to that reported in other studies.

Data from the retrospective study presented in this thesis have shown that the outcome of patients with uncontrolled variceal haemorrhage is dismal and TIPSS in this situation improves survival (Jalan et.al. 1995). This impression has been confirmed by two other group of investigators and most authorities believe that TIPSS is the treatment of choice for patients with uncontrolled variceal hemorrhage (McCormick et.al. 1994, Sanyal et.al. 1996). No significant difference in mortality was detected in the trial comparing TIPSS with VBL for the secondary prophylaxis of variceal 
haemorrhage despite marked differences in rebleeding (Jalan et al. 1997).

This may be related to the design of the study. The primary object of this study was to detect differences in rebleeding, and it was not thought ethical to withhold TIPSS in cases of VBL failure and uncontrolled variceal haemorrhage. TIPSS rescue of patients in the VBL group with uncontrolled variceal haemorrhage may have masked a survival benefit, as 6 of the 8 patients thus treated, survived. The primary reason that Garcia-Villareal's (1996) study comparing TIPSS with sclerotherapy showed a significant reduction in both rebleeding and mortality in the TIPSS arm was the study design, by which patients with uncontrolled variceal haemorrhage in the sclerotherapy arm were not treated with emergency TIPSS. In all the other trials patients with uncontrolled variceal haemorrhage were rescued with TIPSS. Furthermore, no significant differences in mortality in patients with Child $A$ and $B$ or Child class $C$ disease between the two groups highlights the fact that TIPSS does not adversely affect outcome.

Worsening of encephalopathy after TIPSS is independently predicted by its presence prior to TIPSS (Jalan et.al. 1995, Sanyal et.al. 1994). Although insertion of TIPSS precipitated worsening or new onset encephalopathy in 5 patients, this was not significantly different to the frequency of encephalopathy occurring in the VBL group (3 patients). Encephalopathy may be due to progression in liver disease in addition to increased portacaval shunting. The observation that encephalopathy developed late in 1 patient in the TIPSS group and 2 patients in the VBL group suggests that encephalopathy is due at least in part to the deterioration 
in liver function. Furthermore, all the episodes of encephalopathy were managed successfully using conservative measures. Our results, which show no significant differences in the incidence of encephalopathy, is at variance with most of the other data from randomised studies (Cabrera et.al. 1996, Rossle et.al. 1997, Sanyal et.al. 1997). This is difficult to explain but may be accounted for by the population under study. $26 \%$ and $33 \%$ patients in the TIPSS and VBL groups respectively were encephalopathic at the time of randomisation. The other reason for this difference may stem from the definitions of encephalopathy used. We used the simple clinical criteria defined by Parson-Smith et.al (1957). Use of subtle tests of neuropsychological function may have yielded different results.

Patients in the VBL group who rebled, spent significantly greater time in the intensive care unit compared with the patients who did not rebleed. Although the cost of the procedures was significantly higher in the TIPSS group, the overall total cost of treatment was significantly less in the patients treated with TIPSS compared with VBL. This is due to the longer hospital stay in the patients in the VBL group. Furthermore, if 2.4 patients with variceal bleeding are treated with TIPSS, one episode of variceal bleeding would be prevented. In economic terms, the use of TIPSS will generate a significant saving for every rebleed that is prevented. These results should however, be taken in context. These costs estimate only the direct costs as borne by the National Health Service in the UK. These costs do not take into account the establishment costs, hardware cost, radiology or endoscopy time and the cost of training personnel that would be required to establish a new 
service. In addition, these costs may not be directly applicable to all health systems around the world, but in general terms, procedure costs are higher with TIPSS but hospital admissions are less because of a reduction in rebleeding.

In conclusion, if we take the primary end point of the study as rebleeding, this study has shown that TIPSS is superior to variceal band ligation for the secondary prophylaxis of variceal haemorrhage in patients with cirrhosis. On the other hand, if mortality is taken as the revised end point then, the results of this study can also be interpreted to show the TIPSS is as effective as variceal band ligation plus 'TIPSS rescue'. It is important to remember that our trial compares TIPSS with variceal band ligation which has been shown to be superior to sclerotherapy for prevention of rebleeding. However, this study was designed to look at differences in rebleeding and not at mortality. This study has also shown significant reduction in the total cost of treating a patient with a variceal bleed in patients treated with TIPSS compared with VBL. Randomised, clinical trials of endoscopic treatment, comparing VBL with TIPSS need to be performed including large numbers of patients with the first episode of variceal hemorrhage. Because large numbers of patients will be required if the primary end-point of the study is to be mortality, it should be an international multicentre study. The time of randomisation is crucial as any potential benefit of TIPSS will be progressively less with increasing time lag between the bleed and definitive procedure. We recognise the enormous problems facing the organisers of such a study. 


\section{Uncontrolled variceal haemorrhage}

This study comparing TIPSS and surgery in the treatment of uncontrolled variceal haemorrhage confirms that patients requiring these treatments are extremely ill and mortality will be high whatever the treatment used. This study has the limitation of being retrospective and non-randomised. Nevertheless, factors making this comparison worthwhile and valid are that during the 7 years there has been no change in the capacity of the hospital in dealing with these patients, selection criteria were the same in both periods, the patient groups were similar with respect to the severity of liver disease and its complications and the only treatment prior to July 1991 was surgery and since then the only treatment has been TIPSS. We have tried to eliminate the problem of bias by collection of data by 2 separate observers (RJ and TJ) and reviewing all possible avenues of patient retrieval (endoscopy records, surgical audit system and meticulous search through the ward records).

Seven of the 19 patients in the OT group were thought to be unfit for surgery. This information is of immense importance because, although the patients in the TIPSS group had comparable severity and complications of liver disease the patient was never thought to be too ill for the procedure reflecting the relatively less traumatic nature of TIPSS. Furthermore, analysis of the results for the patients in the 2 groups was made on an intention to treat basis. Early mortality in the TIPSS group was half that of the surgical group. Forty percent mortality in patients with TIPSS is reminiscent of the 
figure associated with first bleed (Smith JL and Graham DY 1981, Burroughs et.al. 1989) but rates of $79 \%$ (surgical group) is similar to the figures reported by most groups in this patient population (McCormick et.al. 1994, Teres et.al. 1987b). Perioperative death in the TIPSS group, related to puncture of the portal vein is unusual (LaBerge et.al. 1993, Rossle et.al. 1994, Jalan et.al. 1994) and close attention to the depth of puncture in patients with small fibrotic livers may reduce the risk further. Increase in intracranial pressure in patients with chronic liver disease is rare (Crippin et.al. 1992) and its occurrence in 2 patients in our study is noteworthy. Variceal bleeding, especially if associated with hypotension could cause deterioration in liver function. The high incidence of sepsis in both groups of patients is worrying and probably reflects the population of patients being studied. Most of the patients have advanced forms of liver disease, have been seriously ill, have had numerous procedures including repeated sclerotherapy and balloon tamponade and multiple punctures for peripheral and central venous access for transfusions.

That TIPSS can be used as an effective bridge to transplantation has been well described by Ring et.al. (1992) and is illustrated by the 2 patients, one in the OT group who died waiting to be transferred for transplantation and the other who had a successful TIPSS before transplantation. The case of the patient who underwent transection and later required TIPSS for recurrent variceal haemorrhage and the other who died from recurrent bleeding in the surgical group illustrates the risk of late rebleeding following transection. The high rates of mortality from variceal rebleeding in the OT 
group is noteworthy and in sharp contrast to the results of rebleeding following TIPSS which is usually early and due to shunt insufficiency and can be corrected easily in most cases.

Surgical intervention does however, remain the treatment of choice for patients with extrahepatic portal vein obstruction. Assessment of the patency of the portal vein is necessary in all patients with uncontrolled variceal haemorrhage. The sluggish and reversed portal blood flow may make this assessment difficult. This is illustrated by the patient who was wrongly diagnosed as having portal vein obstruction.

The results of this study indicate that TIPSS can be performed successfully in very ill patients who are not fit to undergo OT and is associated with significantly lower rates of early mortality. In this group of very ill patients, it prolongs life and we believe, should be regarded as the preferred mode of treatment as compared with OT for uncontrolled variceal haemorrhage. A randomised prospective study comparing these treatments is needed to answer this question definitively.

\subsection{SODIUM HANDLING AND TIPSS}

The present study addresses the changes in renal handling of sodium and the possible mechanisms associated with these changes and shows that TIPSS is an effective method of treating ascites in patients with cirrhosis. TIPSS was inserted in the majority of patients for recurrent variceal haemorrhage refractory to sclerotherapy who also had ascites rather than for 
ascites that was difficult to treat. Although ascites was controlled in the 2 patients having TIPSS for refractory ascites, both patients died of progressive liver failure within the first 6 months of TIPSS. The experience of TIPSS in the setting of refractory ascites, remains discouraging. Ochs et.al. (1997) in an uncontrolled study of 50 patients concluded that, although there were significant improvements in $\mathrm{UNa}$ and $\mathrm{CrCl}$, mortality from the underlying liver disease was substantial. Quiroga et.al. (1995) showed that the effect of TIPSS for refractory ascites was also disappointing with about $40 \%$ patients dying within the 6 months without OLT. In a study comparing TIPSS with paracentesis, Lebrec et.al.(1994) concluded that TIPSS was effective only for patients with milder forms of liver disease perhaps due to temporary deterioration in liver failure that often follows insertion of TIPSS. The exact mechanism of this is unclear although the phenomenon of diversion of blood from the liver, constituting 'sinusoidal steal' has been blamed (Jalan et.al. 1995).

The mechanism whereby sodium handling improves after TIPSS is multifactorial. We have shown that although there is a significant reduction in PRA, All and PPG, none of these correlate with the changes in $\mathrm{UNa}$ and $\mathrm{CrCl}$. $\mathrm{LiCl}$ was used as a measure of sodium reabsorption proximal to the inner medullary collecting duct. The mechanism by which TIPSS may modulate changes in $\mathrm{LiCl}$ and ANP is suggested in a study by Quiroga et.al. (1995) who showed that there was an increase in the pre-load and that the norepinephrine concentrations were reduced after TIPSS. The former observation would account for the change in ANP and the latter for an 
increase in $\mathrm{LiCl}$ (Morali et.al. 1991). It is unlikely that the improvement in $\mathrm{LiCl}$ was due to the change in $\mathrm{CrCl}$ following TIPSS because there was no significant correlation between these following TIPSS.

cGMP measurements in this study probably reflects the activity of other unmeasured natriuretic factors and probably nitric oxide activity (Billiar et.al. 1992) because the change in ANP which is the other major determinant of cGMP levels were both not significantly different prior to TIPSS and did not change significantly thereafter.

This study has failed to demonstrate any relationship between the change in $\mathrm{UNa}$ and $\mathrm{CrCl}$ and Pugh score. This finding is supported by Moreau et.al. (1993) who found no correlation between renal blood flow and the severity of liver disease as assessed by antipyrine clearance. Wong et.al. (1994) do however, suggest a relationship between the ability of the kidneys to handle a sodium load due to a specific tubular defect and the severity of liver disease.

It would have been interesting to study serial changes in the renal sodium, hormonal profile and the portal pressure gradient. It was however, thought unethical to catheterise the shunt outside of routine surveillance procedure. A recent abstract (Wong et.al. 1995) did however, address the issue of serial changes in UNa and concluded that portal hypertension and renin-angiotensin axis are important in the pathogenesis of sodium retention in cirrhosis and changes in the renal handling of sodium are not due to changes in the renal haemodynamics, implicating a renal tubular defect. This 
study did however have the limitation of not having concurrent measurements of the portal pressure gradient.

We have failed to demonstrate any direct relationship between the degree of change in UNa and the change in PPG. Support for this observation is provided by Moreau et.al (1993) although differing opinions exist (Epstein et.al. 1977). It does appear that although there is a role for portal hypertension in the aetiology of renal dysfunction in liver disease, the absolute PPG does not appear to be important. It is likely that the renal dysfunction in cirrhosis is multifactorial.

In conclusion, we have demonstrated that significant changes in UNa and $\mathrm{CrCl}$ after TIPSS is due both to changes in the renal proximal tubular function and a reduction in the neurohumoral factors that are involved in sodium retention. The degree of improvement in $\mathrm{UNa}$ and $\mathrm{CrCl}$ were independent of the severity of underlying liver disease and the change in PPG.

\subsection{HEPATORENAL REFLEX}

This study shows that there is a dramatic reduction in RBF following acute increase in the PAG. This change is not related to alteration in the mean arterial pressure, heart rate or the right atrial pressure. The relationship between changes in RBF and cardiac output would ideally have been studied in the same patient but the introduction of a third line for central venous access was thought to be unethical. Although changes in cardiac output (- 
$10.9 \%( \pm 6.9))$ were statistically significant this change was not sufficient to explain the magnitude of the change in RBF $(-43.5 \%( \pm 25.3))$. Furthermore, the changes in cardiac output were not associated with any significant disturbance in the mean arterial pressure which is of paramount importance in the perfusion of the kidneys.

Several theories regarding the initiation of renal vasoconstriction in cirrhosis have been proposed. The underfill hypothesis (Witte et.al. 1971) gave way to the overfill hypothesis (Leiberman et.al. 1970), which has been replaced by the peripheral vasodilatation theory (Schrier et.al. 1988). The initiating event according to the peripheral vasodilatation theory, is splanchnic vasodilatation and portal hypertension produced by the action of splanchnic vasodilators upon the portal and systemic circulation due to reduced clearance and shunting of these substances from the portal to systemic circulation through the collaterals. This causes relative underfilling of the arterial vascular compartment, thereby activating compensatory mechanisms which are responsible for reduction in renal blood flow and sodium retention.

RBF is regulated by intrarenal, humoral and neural factors. The intrarenal factors that have been implicated include the kallikrein-kinin system (MacGilchrist et.al. 1994), prostaglandins (Rimola et.al. 1986), thromboxane, leukotrienes (Moore et.al. 1990), platelet activating factor (Caramelo et.al. 1987), endothelin (Uchihara et.al. 1992), nitric oxide (Lee et.al. 1993) and adenosine (Llach et.al. 1993). Humoral factors that have significant relationship to sodium retention include changes in the renin-aldosterone- 
angiotensin axis (Bosch et.al. 1980), antidiuretic hormone (Moreau et.al. 1994) and the atrial natriuretic peptide (Gines et.al. 1988).

The kidneys are innervated by sympathetic nerves derived from T11, $\mathrm{T} 12$ and $\mathrm{L} 1$ via the coeliac and the aortorenal ganglia. These are distributed both to the renal microvasculature and to the different parts of the nephron (Bajaras et.al. 1984). Stimulation of the sympathetic system produces an increase in the renal vascular resistance thereby reducing RBF (Dibona GF 1987, DiBona GF and Swain LL 1985). The importance of lumbar sympathetic discharge in the pathogenesis of the hepatorenal syndrome is reflected by marked improvement in effective plasma flow following sympathectomy (Solis-Herruzo et.al. 1987). The inverse relationship between portal vein flow and RBF, and the reduction in RBF with acute increase in PAG and renal resistance suggests the existence of a signaling mechanism between the liver and the kidney.

TIPSS has been shown to be effective in the treatment of the hepatorenal syndrome (Ochs et.al 1994, Alam et.al. 1995). The mechanism of this improvement is not entirely clear but the results of this study provides some clues. The reduction in portal pressure gradient by the introduction of TIPSS may be envisaged to increase RBF through the hepatorenal axis and therefore improve renal perfusion, creatinine clearance and sodium excretion.

Ideally, the systemic samples for the measurement of neurohumoral factors should have been collected from the pulmonary artery to avoid the problems of streaming and inadequate mixing. This would involve the 
introduction of a third catheter, which was considered unethical. This streaming effect and poor mixing may be responsible for the higher values of ANP found in the renal vein rather than in the right atrium (ANP is produced in orificium of the coronary sinus and then secreted into the right atrium). Similarly, the kidneys are important in the production of renin. Higher levels of renin are therefore expected in the renal circulation. The finding of higher values of renin in the right atrium in this study may be related to the mixing problem.

Higher cGMP concentration in the systemic circulation probably reflects increased nitric oxide activity because the ANP levels were normal. Normal adrenaline and noradrenaline concentrations in these patients may reflect adequate shunt function and the absence of ascites. Significantly higher concentrations of noradrenaline (vasoconstrictor) and cGMP (reflecting nitric oxide activity - vasodilator) in the renal circulation may represent the reciprocal role of these substances in the regulation of RBF. Insignificant change in the ANP argues against the change in RBF being due to a significant alteration in the circulating volume. Similarly, the renin angiotensin system has not been implicated in the reduction in renal blood flow following acute increase in portal pressure gradient. These observations are against a significant role of these humoral factors in modulating the change in RBF and point to the existence of a hepatorenal axis in the mediation of this phenomenon.

It was therefore somewhat surprising that the levels of adrenaline and noradrenaline were not significantly different prior to and following shunt 
occlusion. This may either be due to the limit of detectability of the assay used or to the rapid uptake and breakdown of these catecholamines by synaptic mono-amine-oxidase inhibitors and catecholamine-o-methyl transferases. The problems of streaming and inadequate mixing of blood in the renal vein and the right atrium may be partially responsible for the normal values of adrenaline and noradrenaline observed. Electrophysiological or pharmacological studies are required for the confirmation of the role of the sympathetic system as an effector arm of this phenomenon. It is also possible that this phenomenon may be mediated by other intrarenal factors described above. Further studies are required to ascertain the role of these factors in the mediation of this phenomenon which may be important in the pathogenesis of the hepatorenal syndrome.

The contribution of the reduction in cardiac output following shunt occlusion to the reduction in RBF cannot be disregarded. Occlusion of TIPSS appears to induce a decrease in venous return and therefore, possibly redistribution in blood flow. However, the change in cardiac output did not produce any significant haemodynamic disturbance. The transient increase in RBF between 2 and 4 minutes in 3 patients following inflation of the balloon is difficult to explain but further argues against a significant role of reduction in $\mathrm{CO}$ being important in the change in RBF. Unfortunately, in this study we have not measured changes in $\mathrm{CO}$ at these time points. The statistical insignificance of the change in mean arterial pressure is unlikely to be due to the number of patients studied in each group because the difference remained inconsequential even when changes in both groups were 
considered together. Finally, as pointed out above, the degree of reduction in CO cannot fully explain the changes in RBF.

In conclusion, we have demonstrated a dramatic reduction in RBF with acute increase in PAG which is compatible with the existence of hepatorenal reflex in man, although other mechanisms may also be contributory. This is a reproducible model which can be used to study this phenomenon further, enhance our understanding of the changes in renal circulation in liver disease thereby, providing clues for the development of newer treatments. 


\subsection{OVERALL CONCLUSIONS}

The introduction of TIPSS into clinical practice has provided an exciting new modality of treatment that is widely applicable to treat the most dreaded complication of portal hypertension, i.e. variceal haemorrhage. Although there is little doubt about the role of TIPSS in the management of uncontrolled variceal haemorrhage in patients with cirrhosis, further larger studies are needed to determine the role of TIPSS in the secondary prophylaxis of variceal haemorrhage in patients with cirrhosis. TIPSS produces significant improvement in sodium excretion in patients with cirrhosis but its use in patients with refractory ascites and hepatorenal failure require further evaluation.

The 'Achilles heel' of TIPSS is shunt insufficiency. Innovative methods such as the use of 'covered stents' and shunt brachytherapy require further evaluation. Transient deterioration of liver function after TIPSS occurs in about $40 \%$ patients and patient selection to determine which patients are likely to die within a few weeks of TIPSS requires clarification. The incidence of encephalopathy after TIPSS is elevated and the use of further modifications of the type of stent, such as those that can produce an end-toside portacaval shunt may be necessary to prevent this complication.

Finally, and perhaps most importantly, TIPSS provides repeated access to the portal system and will allow understanding of the pathogenesis of portal hypertension and hepatic encephalopathy. 


\section{REFERENCES}

Alam I, Bass NM, LaBerge JM, Ring EJ, Somberg KA Treatment of hepatorenal syndrome with the transjugular intrahepatic portosystemic shunts (TIPS). Gastroenterology 1995; 108:1024A (abstract)

Alexandrino PT, Alves MN, Correia JP. Propranolol or endoscopic sclerotherapy in the prevention of recurrence of variceal bleeding: a prospective randomized control trial. Hepatology 1988;7:175-185

Andreani T, Poupon RE, Balkau BJ, Trinchet JC, Grange JD, Peigney N, et al. Preventive therapy of first gastrointestinal bleeding in patients with cirrhosis: Results of a controlled trial comparing propranolol, endoscopic sclerotherapy and placebo. Hepatology 1990; 12:1413-1419.

Angelico M, Carli L, Piat C, Gentile S, Rinaldi V, Bologna E, et al. Isosorbide5 -mononitrate versus propranolol in the prevention of first bleeding in cirrhosis. Gastroenterology 1993; 104:1460-1465.

Arroyo V, Gines P, Gerbes AL, Dudley FJ, Gentilini P, Laffi G, Reynolds TB, RingLarsen $\mathrm{H}$, Scholmerich J Definition and diagnostic criteria of refractory ascites and hepatorenal syndrome in cirrhosis Hepatology 1996;23:164-176

Arroyo V, Planas R, Gaya J et al. Sympathetic nervous activity, reninangiotensin system and renal excretion of prostaglandin $\mathrm{E}$ in cirrhosis related to functional renal failure and sodium and water excretion. European Journal of Clinical Investigation 1983;13: 271-2778.

Asbert M, Gines A, Gines P, Jimenez W et al. Circulating levels of endothelin in cirrhosis. Gastroenterology 1993;104:1485-1491.

Avgerinos A, Klonis C, Rekoumis g, gouma $P$, Papadimitriou N, Raptis S. A prospective randomised trial comparing somatostatin, balloon tamponade and the combination of both methods in the management of acute variceal haemorrhage. J Hepatol 1991;13:78-83

Avgerinos A, Armonis A, Manolakopoulos S, Poulianos G, Rekoumis G, Sgourou $A$, et al. Endoscopic sclerotherapy versus variceal ligation in the long-term management of patients with cirrhosis after variceal bleeding. A prospective randomized study. Journal of Hepatology 1997; 26:1034-1041

Bagarani M, Albertini V, Anza M, Barlattani, Bracci F, Cucchiara G, Gizzonio $D$ et al. Effect of somatostatin in controlling bleeding from esophageal varices. Ital J Surg Sci 1987;17:21-26

Bailar III JC The promise and problems of meta-analysis. $\mathrm{N}$ Eng J Med 1997;337:559-561 
Baker LA, Smith C, Lieberman G. The natural history of esophageal varices. American Journal of Medicine 1959;26:228-237

Balanzo J, Villanueva C, Espinos J et al. Predictive value of the endoscopic signs in variceal bleeding. Journal of Hepatology 1991;13:(suppl 2):S93 (abstract)

Baldus W P. Etiology and management of renal failure in cirrhosis and portal experiment. Ann N Y Acad Sci 1970;170: 267-279

Baldus, W.P., Summerskill, WHJ, Hunt, J.C., Maher, F.T. Renal circulation in cirrhosis: observations based on catheterisation of the renal vein. J.Clin Invest 1964; 43: 1090-97

Ballet $F$, Chretien $Y$, Rey $C$ et al. Differential response of normal and cirrhotic liver to vasoactive agents. A study in the isolated perfused liver. Journal of Experimental Pharmacology and Therapeutics 1988;244: 283-289

Barajas, L., Powers, K., Wang,P. Innervation of the renal cortical tubules: a quantitative study. Am.J.Physiol 1984;247: F50-F60

Baraldi M, Zeneroli ML, Ricci P, Ventura E. Down regulation of striatal dopamine receptors in experimental hepatic encephalopathy. Life Sci 1983; 32: $1417-1425$

Barsoum NS, Boulous FI, Ell-Robby A, et al. Tamponade and injection sclerotherapy in the management of bleeding oesophageal varices. $\mathrm{Br} J$ Surg 1982;69:76-78

Basile AS, Jones EA, Skolnick, $P$. The pathogenesis and treatment of Hepatic Encephalopathy: Evidence for the involvement of Benzodiazepine receptor Ligands. Pharmacological reviews 1991;43:27-71

Basile AS, Jones EA. Ammonia and GABAergic neurotransmission: Interrelated factors in the pathogenesis of hepatic encephalopathy. Hepatology 1997;25:1303-1305

Benoit JN, Zimmerman B, Premen AJ, et.al. Role of Glucagon in the splanchnic hyperaemia of chronic portal hypertension. Am J Physiol 1986;251:G674-G677

Beppu K, Inokuchi K, Koyanagi N, et al. Prediction of variceal hemorrhage by esophageal endoscopy. Gastrointestinal Endoscopy 1981; 27:213-218 
Bhathal P S and Grossmann H J. Reduction of the increased portal vascular resistance of the isolated perfused cirrhotic rat liver by vasodilators. Journal of Hepatology 1985;1: 325-337

Billiar TR, Curran RD, Harbrecht BG, Stadler J, Williams DL, Ochoa JB, DiSihio $M$, Simmons RL, Murray SA. Association between synthesis and release of cGMP and nitric oxide biosynthesis by hepatocytes. Am J Physiol 1992;262:1077-82

Bioulac-Sage $P$, Lafon $M E$, Saric $J$ and Ballabaud $C$. Nerves and perisinusoidal cells in human liver (Review). Journal of Hepatology 1990;10:105-112

Blei A T, Granger D, Fung $H$ L et al. Organic nitrates in portal hypertension. European Heart Journal 1988;9A: 205-211

Blendis L M, Orrego $H$, Crossley I $R$ et al. The role of hepatocyte enlargement in hepatic pressure in cirrhotic and non-cirrhotic alcoholic liver disease. Hepatology 1982;2: 539-546

Bosch J, Arroyo V, Betriu A, Mas A, Carrilho F, Rivera F, Navarro-Lopez F, Rodes J. Hepatic hemodynamics and the renin-angiotensin-aldosterone system in cirrhosis. Gastroenterology 1980;78:92-99

Bosch J, Mastai R, Kravetz D, et.al. Effects of propranolol on azygos blood flow and hepatic and systemic haemodynamics in cirrhosis. Hepatology 1984;6:1200-1205

Bosch J, Bordas JM, Mastai R, et al. Effects of vasopressin on the intravariceal pressure in patients with cirrhosis: comparison with the effects on portal pressure. Hepatology 1988;8:861-865

Bosch J, Groszman RJ, Garcia-Pagan JC, Teres J, Garcia-Tsao G, Navasa $M$, Mos $A$ et al. Association of transdermal nitroglycerin to vasopressin infusion in the treatment of variceal hemorrhage: a placebo-controlled clinical trial. Hepatology 1989;10:962-968

Bosch J, Kravetz D, Mastai R, et al. Effects of somatostatin in patients with portal hypertension. Hormone Res 1989;29:99-102

Bosch J, Kravetz D, Rodes J. Effects of somatostatin on hepatic and systemic hemodynamics in patients with cirrhosis of the liver. Comparison with vasopressin. Gastroenterology 1981:80-518-525 
Bosch M, Jiminez W, Angeli P, Leivas A, Claria J, Graziotti A et al. Acuaretic efficacy of the K-opioid receptor against RU 51599 in cirrhotic rats with ascites. Journal of Hepatology 1994; 21: S7 (abstract)

Boyer T.D., Triger D.R., Horisawa M., et.al.Direct transhepatic measurement of portal vein pressure using a thin needle. Comparison with wedged hepatic vein pressure. Gastroenterology 1977; 72:584-89

Brensing KA, Textor J, Strunk $H$, Klehr HU, Schild $H$, Sauerbruch T Transjugular intrahepatic portosystemic stent-shunt for hepatorenal syndrome. Lancet 1997;349:697-698

Burgener FA, et.al. Nonsurgical production of intrahepatic portosystemic shunt venous shunt in portal hypertension with double lumen balloon catheter. ROFO 1979;130:686

Burgener FA. Gutierrez OH. Experimental intrahepatic portocaval shunts created in portal hypertension by balloon angioplasty catheters. Investigative Radiology 1988; 23:24-29

Burghardt W, Wernze H, Diehl K L. Atrial natriuretic peptide in cirrhosis: relation to stage of disease, sympathoadrenal system and renin-aldosterone axis. Klin Wochenschr 1986;64 (suppl VI): 103-107

Burroughs AK, d'Heygere F, Mclntyre N. Pitfalls in studies of prophylactic therapy for variceal bleeding in cirrhotics. Hepatology 1986;6:1407-1413

Burroughs AK, Hamilton G, Phillips A, et al. A comparison of sclerotherapy with staple transection of the esophagus for the emergency control of bleeding from esophageal varices. N Engl J Med 1989;321:857-862

Burroughs AK, Jenkins WJ, Sherlock S et al . Controlled trial of propranolol for the prevention of recurrent variceal hemorrhage in patients with cirrhosis. N Engl J Med 1983;309:1539-1542

Burroughs AK, McCormick DA, Siringo S, Phillips A, Mclntyre N. Prospective randomized trial of long term sclerotherapy for variceal rebleeding, using the same protocol to treat rebleeding in all patients. Final report. $\mathrm{J}$ Hepatol $1989 ; 9$ (suppl)S12

Burroughs AK, McCormick PA, Hughes MD, Sprengers D, DHeygere $F$, McIntyre N. Randomized, double-blind, placebo-controlled trial of somatostatin for variceal bleeding: Emergency control and prevention of early variceal rebleeding. Gastroenterology 1990; 99:1388-1395 
Burroughs AK, McCormick PA, Siringo S, et al. Prospective randomized trial of long-term sclerotherapy for variceal bleeding using the same protocol to treat rebleeding in all patients (Abstract). J Hepatol 1989;9:S12

Burroughs AK, Mezzanote G, Phillips A, Aiden McCormick P, Mclntyre N. Cirrhotics with variceal haemorrhage: The importance of the time interval between admission and the start of analysis for survival and rebleeding rates. Hepatology 1989;9:810-807

Burroughs AK, Sclerotherapy of varices. In: Rodes J, Arroyo, V. eds. Therapy in liver disease. Barcelona. Ediciones Doyma 1992;114-127

Burroughs AK. Somatostatin and octreotide for variceal bleeding. J Hepatol 1989;13:1-4.

Burroughs AK. Double blind randomised clinical trial of 5 day octreotide versus placebo associated sclerotherapy for treatment failures. Hepatology 1996;24:901 (abstract)

Burroughs AK. The natural history of varices. Journal of Hepatology 1993; 17:S10-S13.

Butterworth RF A disorder of multiple neurotransmitter systems Advances in Experimental Medicine and Biology 1994;368:79-88

Butterworth, R.F. Evidence that hepatic encephalopathy results from a defect of glutamatergic synaptic regulation. Mol. Neuropharmacol. 1992;2: 229-232.

Butterworth R.F. (1995). Neurobiology and neuropharmacology of hepatic encephalopathy. In: Capocaccia, L., Merli, M., and Riggio, O. (Eds). Advances in Hepatic Encephalopathy and Metabolic Nitrogen Exchange. CRC Press, Boca Raton, Florida, ch 19, pp 99-108.

Butterworth, R.F., Giguere, J.F., Michaud, J., Lavoie, J., and PomierLayrargues, G. Ammonia: key factor in the pathogenesis of hepatic encephalopathy. Neurochem Pathol. 1987;6: 1-12.

Cabrera J, Maynar M, Granados R, Gorriz E, Reyes R, PulidoDuque JM, SanRoman JLR, Guerra C, Kravetz D Transjugular intrahepatic portosystemic shunt versus sclerotherapy in the elective treatment of variceal hemorrhage Gastroenterology, 1996;110:832-839

Cales P, Pascal JP. Natural history of esophageal varices in cirrhosis (from origin to rupture). Gastroenterologie Clinique et Biologique 1990; 12:245-254. 
Cales P, Quinton A, Lamouliatte H, Paccalin J, Dabadie H, Tournut R, et al. Predictive factors for the first bleeding event and death in cirrhotic patients with esophageal varices. Gastroenterologie Clinique et Biologique 1989; 13:54-59.

Calver A, Harris A, Maxwell J D, Vallence PEffect of local inhibition of nitric oxide synthesis on forearm blood flow and dorsal hand vein size in patients with alcoholic cirrhosis. Clinical Science, 1994;86:203-208.

Caramelo C, Fernandex-Gallardo S, Santos J C et al. Increased levels of PAF-acether in blood from patients with cirrhosis of the liver. European Journal of Clinical Investigation, 1987;17: 7-11.

Cardona C, Vida F, Balanzo J, Cusso X, Farre A, Guarner C. Eficacia terap $\square$ utica de la somatostatina versus vasopressina mas nitroglycerina en la hemorragia activa por varices esofagogastrica. Gastroenterol Hepatol 1989;12:30-34.

Cardin F, Gori G, McCormick PA, Burroughs AK. A predictive model for very early rebleeding from varices. Gut 1990;31:A1204 (abstract)

Castro A, Jimenez W, Claria J, Ros J, Martinez J M et al. Impaired responsiveness to angiotensin $\|$ in experimental cirrhosis: role of nitric oxide. Hepatology, 1993;18: 367-372.

Cello JP, Grendell JH, Crass RA, Weber TE, Trunkey D. Endoscopic sclerotherapy versus portacaval shunt in patients with severe cirrhosis and acute variceal hemorrhage. Long term follow-up. N Engl J Med 1987;316:1115.

Cello JP, Ring EJ, Olcott EW, Koch J, Gordon R, Sandhu J, Morgan DR, Ostroff JW, Rockey DC, Bacchetti P, LaBerge J, Lake JR, Somberg K, Doherty C, Davila M, McQuaid K, Wall SD Endoscopic sclerotherapy compared with percutaneous transjugular intrahepatic portosystemic shunt after initial sclerotherapy in patients with acute variceal hemorrhage: $A$ randomized, controlled trial Annals of Internal Medicine, 1997;126:858-865

Cello JP,Crass R, Trunkey DD. Endoscopic sclerotherapy versus esophageal transection in Child's class $C$ patients with variceal hemorrhage. Comparison with results of portacaval shunt. Preliminary report. Surgery 1982;91:333-338.

Cerbelaud $P$, Lavignolle $A$, Perrin D, Jutel P, Beaujard E, Colomb $P$, Le Bodic L. Propranolol et prevention des recidives de rupture de varice oesophagienne du cirrhotique. Gastroenterol Clin Biol 1986;18:A10. 
Chiu WK, Sheen IS, Liaw YR. A controlled study of glypressin versus vasopressin in the control of bleeding from esophageal varices. J Gastroenterol Hepatol 1990;5:549-553.

Chou C C and Kvietys P R. (1981) Physiological and pharmacological alterations in gastrointestinal blood flow. In Granger D N and Buckley $G$ B (eds). Measurement of Blood Flow, Applications to the Splanchnic Circulation. Baltimore: Williams and Wilkins.

Christensen E, Fauerholdt L, Schlichting P, et al. Aspects of the natural history of gastrointestinal bleeding in cirrhosis and the effect of prednisone. Gastroenterology 1981; 81:944-952.

Christensen E, Krintel JJ, Hansen M, Krogh S et al. Prognosis after the first episode of gastrointestinal bleeding or coma in cirrhosis. Scandinavian Journal of Gastroenterology 1989;24:999-1006.

Claria J, Jimenez W, Arroyo V, La Villa G et al. Effect of V1-Vasopressin receptor blockade on arterial pressure in conscious rats with cirrhosis and ascites. Gastroenterology 1991;100:494-501

Colapinto,R.F., $\quad$ Stronell,R.D., $\quad$ Gildiner,M., Ritchie,A.C., Langer,B., Taylor,B.R., Blendis,L.M. Formation of an intrahepatic portasystemic shunt using ballon dilataton catheter:Preliminary clinical experience. A.J.R. Am.J.Roentgenol. 1983;140:709-14

Colin R, Giuli N, Czernichow P, Ducrotte P, Lerebours E. Prospective comparison of glypressin, tamponade and their association in the treatment of bleeding esophageal. In: Lebrec D, Blei AT, eds. Vasopressin analogs and portal hypertension. John Libbey Eurotext, Paris, 1987:149-153

Colman J, Jones P, Finch C, Dundley F. Propranolol in the prevention of variceal hemorrhage in alcoholic cirrhotic patients (Abstract). Hepatology 1990;12:851.

Colombo M, De Franchis R, Tommasini M, Sangiovanni A, Dioguardi N. beta-Blockade prevents recurrent gastrointestinal bleeding in wellcompensated patients with alcoholic cirrhosis: A multicenter randomized controlled trial. Hepatology 1989; 9:433-438.

Conn HO, Lindenmuth WW, May CJ, Ramsby GR. Prophylactic portacaval anastomosis in cirrhotic patients with esophageal varices. $\mathrm{N}$ Eng J Med $1965 ; 272: 1255-1263$

Conn HO, Bircher J, eds. Hepatic encephalopathy: management with lactulose and related carbohydrates. East Lansing, Medi-Ed Press, Michigan, 1988; 199-206. 
Conn HO, Grace ND, Bosch J, Groszmann RJ, Rodes J, Wright SC, Matloff DS, GarciaTsao G, Fischer RL, Navasa M, Drewniak SJ, Atterbury CE, Bordas JM, Lerner E, Bramante C. Propranolol in the prevention of the first haemorrhage from oesophagogastric varices. A multicenter randomised clinical trial. 1991;13:902-912

Conn HO, Lieberthal MM. The hepatic coma syndromes and lactulose. Baltimore:Williams and Wilkins, 1979

Conn HO, Lindenmuth WW, May CJ, Ramsby GR. Prophylactic portacaval anastomosis. A tale of two studies. Medicine 1972;51:27-40.

Conn HO, Ramsby GR, Storer EH, Mutchnick MG, Joshi PH, Phillips MM, Cohen GA et al. Intraarterial vasopressin in the treatment of upper gastrointestinal hemorrhage: a prospective controlled clinical trial. Gastroenterology 1975;68:211-221.

Conn $\mathrm{HO}$. Adverse reactions and side effects of lactulose and related agents. In:

Conn, H.O. (1992). Assessment of the severity of hepatic encephalopathy. In: Rodes, J., and Arroyo, V. (Eds). Therapy in Liver Diseases. Ediciones Doyma, Barcelona, VII, pp 277-291.

Conn, H.O. Trail making and number-connection tests in the assessment of mental state in portal systemic encephalopathy. Am J Dig Dis. 1977; 22: 541-550.

Conn, H.O., Leevy, C.M., Vlahcevic, Z.R., Rodgers, J.B., Madrey, W.C., Seeff, L., and Leevy, L.L. Comparison of lactulose and neomycin in the treatment of chronic portal-systemic encephalopathy: a double blind trial. Gastroenterology. 1977; 72: 573-583.

Cordoba J, Gottstein J, Blei AT. Glutamine, myo-inositol and organic brain osmolytes after portacaval anastomosis in the rat. implications for ammonia induced brain edema. Hepatology 1996;24:919-923

Cox DR. Regression models and life tables (with discussion). J R Stat Soc. 1972; 34: 187-204.

Crippin, J.S., Gross, J.N.R., Lindor, K.D.Increased intracranial pressure and hepatic encephalopathy in chronic liver disease. Am. Jnl. Gastro. 1992;15:403-406

Crossley I R, Westaby D and Williams R. (1985) Portal hypertension. In Wright R and Milward-Sadler G H (eds). Liver and Biliary Disease, pp 12911293. London: Bailliere Tindall. 
Czaja AJ, Wolf AM, Summerskill WH. Development and early prognosis of esophageal varices in severe chronic active liver disease (CALD) treated with prednisolone. Gastroenterology 1979;77:629-633.

D'Amico G, Pagliaro L, Bosch J. The treatment of portal hypertension: A meta analytical review. Hepatology 1995;22:332-353

D'Amico G, Traina M, Vizzini G, Tine F, Politi F, Montalbano L, Luca A et al. Terlipressin or vasopressin plus transdermal nitroglycerin in a treatment strategy for digestive bleeding in cirrhosis. A randomized clinical trial. J Hepatol 1994;20:206-212.

Dabos, K., Stanley, A.J., Jalan, R., Redhead, D.N., Hayes, P.C. Shunt insufficiency after TIPSS: Balloon angioplasty or insertion of parallel shunt. Gut (supple)1996; W12 (abstract).

Dagradi A. The natural history of oesophageal varices in patients with alcoholic liver disease. Am J of Gastroenterol 1972;57:520-540.

Darcy M D, Veseley T M, Picus D, Middleton W D, Hicks M E. Percutaneous revision of an acutely thrombosed transjugular intrahepatic portosystemic shunt. J Vasc Intervent Radiol 1992;3:77-82.

Dasarathy S, Dwivedi M, Bhargava DK, Sundaram KR, Ramachandran K. A prospective randomized trial coparing repeated endoscopic sclerotherapy and propranolol in decompensated (Child class B and C) cirrhotic patients. Hepatology 1992;16:89-94.

Dawson J, Gertsch P, Mosimann F, et al. Endoscopic variceal pressure measurements: Response to isosorbide dinitrate. Gut 1985; 26:843-847.

De Franchis R, Pascal JP, Ancona E, Burroughs AK, Henderson M, Fleig W, et al. Definitions, methodology and therapeutic strategies in portal hypertension. A Consensus Development Workshop, Baveno, Lake Maggiore, Italy, April 5 and 6, 1990. Journal of Hepatology 1992; 15:256-261.

De Franchis $R$, Primignani M, Arcidiacono PG, Rizzi PM, Vitagliano P, Vazzoler MC, et al. Prophylactic sclerotherapy in high-risk cirrhotics selected by endoscopic criteria: A multicenter randomized controlled trial. Gastroenterology 1991; 101:1087-1093.

de Haes, J.C.J.M., van Knippenberg, F.C.E., Neijt, J.P. Measuring psychological and physical distress in cancer patients: structure and application of the Rotterdam Symptom Checklist. British J Cancer 1990; 62: 1034-1038

Desaint B, Florent C, Levy VG. A randomised trial of triglycyl-lysine vasopressin versus lysine vasopressin in active cirrhotic variceal 
hemorrhage. In: Lebrec D, Blei AT, eds. Vasopressin analogs and portal hypertension. John Libbey Eurotext, Paris, 1987;155-157.

DeTroyer A, Pilloy W, Brockaert I, Demanet JD Demeclocycline treatment of water retention in cirrhosis. Ann Intern Med 1976;85:336-337

DiBona, G.F. Neural regulation of renal tubular sodium reabsorption and renin secretion: integrative aspects. Clin.Exp.Hypertens. [A] 1987; 9 (suppl 1):151-165

DiBona, G.F., Sawin, L.L. Renal nerve activity in concious rats during volume expansion and depletion. Am.J.Physiol. 1985;248:F15-F23

Dollet JM, Champigneulle B, Patris A, Bigard MA, Gaucher P. Endoscopic sclerotherapy versus oral propranolol after variceal haemorrhage in cirrhosis: Results of a 4-year prospective randomised trial. Gastroenterologie Clinique et Biologique 1988;12:234-239

Dotter CT et.al. Percutaneous intrahepatic portosystemic shunt for portal hypertension. Proceedings from RSNA, 1981

Epstein M (ed). (1988) The Kidney in Liver Disease. Williams and Williams (3rd edit).

Epstein M, Berk D P, Hollenberg N K, Adams D F, Chalmers T C, Abrahams $H$ L, Merrill J P. Renal failure in patients with cirrhosis. The role of active vasoconstriction. American Journal of Medicine, 1970:49: 175-185.

Epstein M, Larios O, Johnson G. Effects of water immersion on plasma catecholamines in decompensated cirrhosis. Implications for deranged sodium and water homeostasis. Miner Electrolyte Metab 1985B;11:25-34

Epstein M. Derangements of water handling in liver disease. Gastroenterology 1985A;89:1415-1425

Epstein, M., Schneider, N., Befeler, B. Relationship of systemic and intrarenal hemodynamics in cirrhosis. J Lab Clin Med. 1977;89:1175-1187

Fan P, Lavoie J, Le NLO, Szerb JC and Butterworth RF. Neurochemical and electrophysiological studies on the inhibitory effect of ammonium ions on synaptic transmission in slices of rat hippocampus: evidence for a postsynaptic action. Neuroscience 1990; 37: 327-334.

Fauvel. (1858) Bull de la Soc. Med. d'Obs. Cited by Preble 1900.

Ferenci P, Herneth A, Steindl P Newer approaches to therapy of hepatic encephalopathy. Seminars in Liver Disease 1996;16:329-338 
Ferguson, J., Jalan, R., Redhead, D.N., Hayes, P., Allan, P.A.The role of Duplex Doppler in monitoring shunt function following transjugular intrahepatic stent shunt. British J Radiology. 1995;68:587-589

Feu F, Del Arbol LR, Banares R, Planas R, Bosch J. Double-blind randomized controlled trial comparing terlipressin and somatostatin for acute variceal hemorrhage. Gastroenterology 1996; 111:1291-1299.

Fischer JE Branched chain enriched amino acid solutions in patients with liver failure: An early example of nutritional pharmacology Journal of Parenteral and Enteral Nutrition 1990;14:249S-256S

Fischer JE, Baldessarini RJ. False neurotransmitters and hepatic failure. Lancet 1971; 2: 75-80.

Fischer JE, Yoshimura N, James JH et al. Plasma amino acids in patients with hepatic encephalopathy: effects of amino acid infusions. Am J Surg. $1974 ; 127: 40-47$.

Fischer, J.E., (1992) Portal-systemic encephalopathy. In Millward-Sadler, G.H., Wright, R., Arthur, M.J.P. (eds.). Wright's Liver and Biliary Disease. W.B. Saunders Company Ltd., London, pp 1262-1295.

Fisher JE, Bower RH, Atanian S, Welling R. Comparison of distal and proximal splenorenal shunts. Ann Surg 1981;194:531-544.

Fleig WE, Stance EF, Hunecke R, et al. Prevention of recurrent bleeding in cirrhotics with recent variceal hemorrhage: Prospective, randomized comparison of propranolol and sclerotherapy. Hepatology 1987; 7:355-361.

Fogel MR, Knauer CM, Andres LL, et al. Continuous intravenous vasopressin in active upper gastrointestinal bleeding. A placebo-controlled trial. Annals of Internal Medicine 1982; 96:565-569.

Forrest EH, Jalan R, Hayes PC Review article:renal circulatory changes in cirrhosis-pathogenesis and therapeutic prospects Aliment Pharmacol Ther $1996 ; 10: 219-231$

Fort $E$, Sautereau D, Silvaine $C$, Ingrand $P$, Pillegand B, Beauchant M. A randomised trial of terlipressin plus nitroglycerin vs balloon tamponade in the control of acute vasopressin hemorrhage. Hepatology 1990;11:678-681.

Foshager MC, Nazarian GK, Ferral H, et.al. Duplex and colour Doppler sonography in the follow up evaluation of TIPS (abstract) SCVIR, 19th annual meeting progress. 1994;38-39 
Freeman JG, Cobden I, Record CO. Placebo controlled trial of terlipressin (Glypressin) in the management of acute variceal bleeding. $\mathrm{J}$ Clin Gastroenterol. 1989;11:58-60.

Friedman HP, Goldberg JD. Meta-analysis: an introduction and point of view. Hepatology 1996;23:917-928

Garcia-Villareal L, Martinez-Lagares F, Sierra A, Guevara C, HernandezCabrero T, Marrero JM, Jimenez E, Fuentes R, Alonso JM. TIPS vs Sclerotherapy for the prevention of variceal rebleeding. Preliminary results of a randomised study. Hepatology 1996;24:208A (abstract).

GarciaTsao G, Groszmann RJ, Fisher RL, et al. Portal pressure, presence of gastroesophageal varices and variceal bleeding. Hepatology 1985; 5:419424.

Garden OJ, Mills PR, Birnie GG, Murray GD, Carter DC. Propranolol in the prevention of recurrent variceal hemorrhage in cirrhotic patients. A controlled trial. Gastroenterology 1990; 98:185-190.

Garden OJ, Motyl H, Gilmour WH et al. Prediction of outcome following acute variceal haemorrhage. Br J Surg 1985;72:91-95.

Gatta A, Merkel C, Sacerdoti D, Bolognesi M, Caregaro L, Zuin R, Angeli P et al. Nadolol for prevention of variceal rebleeding in cirrhosis: a controlled clinical trial. Digestion 1987;37:22-28.

Genoud E, Gonvers JJ, Schaller MD, et.al. Prognostic value of the reninangiotensin system for the response to salt restriction and prognosis of alcoholic cirrhosis and ascites. Sch. Med Woch, 1986;116:463.

Gerbes AL, Moller S, Gulberg V, Henriksen JH Endothelin-1 and -3 plasma concentrations in patients with cirrhosis: Role of splanchnic and renal passage and liver function Hepatology, 1995;21:735-739

Gimson AES, Ramage JK, Panos MZ, Hayllar K, Harrison PM, Williams R, et al. Randomised trial of variceal banding ligation versus injection sclerotherapy for bleeding oesophageal varices. Lancet 1993; 342:391-394.

Gimson AES, Westaby D, Hegarty J, Alastair W, Williams R. A randomized trial of vasopressin plus nitroglycerin in the control of acute variceal hemorrhage. Hepatology 1986;6:410-413.

Gimson AES, Westaby D, Kravetz D et al. A randomised trial of vasopressin and vasopressin plus nitroglycerin in the control of variceal haemorrhage. Hepatology. 1986;6:410-413. 
Gines A, Escorell A, Gines P, Salo J, Jimenez W, Inglada L, Navasa M, Claria J, Rimola A, Arroyo V, Rodes J. Incidence, Predictive factors, and Prognosis of the hepatorenal syndrome in cirrhosis with ascites. Gastroenterology 1993;105:229-236

Gines P, Arroyo V, Vergas V, Planas R, Casafont F, Panes J, Hoyos M, Vilamodiu L, Rimola A, Morillas R, Salmeron JM, Gines A, Esteban R, Rodes $\mathrm{J}$. Paracentesis with intravenous infusion of albumin as compared with peritoneo-venous shunting in cirrhosis with refractory ascites. N Eng J Med $1991 ; 325: 829-835$

Gines P, Jimenez W, Arroyo V, Navasa M, Lopez C, Tito L, Serra A, Bosch J, Sanz G, Rivera F, Rodes J Atrial natriuretic factor in cirrhosis with ascites; plasma levels, splanchnic release and cardiac extraction. Hepatology 1988;8:636-642.

Gines P, Jimenez W, Arroyo V, Navasa M, Lopez C, Tito L, Serra A, Bosch J, Sanz G, Rivera F, Rodes J Urinary excretion of 6-keto-prostaglandin $\mathrm{F}_{\mathrm{l}}$, thromboxane $B_{2}$, and prostaglandin $E_{2}$ in cirrhosis with ascites; relationship to functional renal failure (hepatorenal syndrome). Journal of Hepatology 1986;3:111-117.

Gines $\mathrm{P}$, Jiminez W, Aroyo $\mathrm{V}$ et al. Atrial natriuretic factor in cirrhosis with ascites; plasma levels, splanchnic release and cardiac extraction. Hepatology, 1988A; 8: 636-642.

Gines P, Tito L, Arroyo V, Planas R, Panes J, Viver J, Torres M, Humbert P, Rimola A, Llach J, Badalamenti S, Jimenez W, Gaya J, Rodes J Randomised comparative study of therapeutic paracentesis with and without intravenous albumin in cirrhosis. Gastroenterology 1988;94:1493-1502

Goldstein, D.S., Feuerstein, G., Izzo, J.L., Kopin, I.J., Keiser, H.R. Validity and reliability of liquid chromatography with electrochemical detection for measuring plasma levels of norepinephrine and epinephrine in man. Life sciences $1981 ; 28: 467-75$.

Gordon JD. Colapinto RF. Abecassis M. et aL. Transjugular intrahepatic portosystemic shunt: A nonoperative approach to life-threatening variceal bleeding. Canadian Journal of Surgery. 1987;30:45-49

Grace ND, Conn HO, Resnick RH, Groszmann RJ, Atterbury CE, Wright SC, Gusberg RJ et al. Distal splenorenal vs portal-systemic shunts after hemorrhage from varices: a randomized controlled trial. Hepatology 1988;8:1475-1481.

Granger D N, Richardson P D I, Kvietys P R et al. Intestinal blood flow. Gastroenterology, 1980; 78: 837-865. 
Gregory PB. Prophylactic sclerotherapy for esophageal varices in men with alcoholic liver disease. A randomized, single-blind, multicenter clinical trial. Annals of Internal Medicine 1991; $115: 73$

Groszmann RJ, Bosch J, Grace ND, Conn HO, GarciaTsao G, Navasa M, et al. Hemodynamic events in a prospective randomized trial of propranolol versus placebo in the prevention of a first variceal hemorrhage. Gastroenterology 1990; 99:1401-1407.

Groszmann RJ, Kravetz D, Bosch J, Glickman M, Bruix J, Bredfelt J, Conn $\mathrm{HO}$ et al. Nitroclycerin improves the hemodynamic response to vasopressin in portal hypertension. Hepatology 1982;2:757-762.

Groupe d'Etude des Anastomoses Intra-Hepatiques (Toulouse, Bondy, Lille, Nice, Creteil, Paris) France. TIPS vs Sclerotherapy + Propranolol in the prevention of variceal rebleeding: preliminary results of a multicenter randomised trial. Hepatology 1995;22:299A (abstract)

Guarner C, Soriano G, Such J, Teixido M, Ramis I et al. Systemic prostacyclin in cirrhotic patients. Gastroenterology, 1992;102: 303-309.

Hadengue A, lee S S, Moreau R, Brallion A et al. Beneficial haemodynamic effects of Ketanserin in patients with cirrhosis: possible role of serotonergic mechanisms in portal hypertension. Hepatology, 1987;7: 644-647.

Harley HAJ, Moergan T, Redeker AG, Reynolds TB, Villamil F, Weiner JM, Yellin A. Results of a randomized trial of end-to-side portacaval shunt and distal splenorenal shunt in alcoholic liver disease and variceal bleeding. Gastroenterology 1986;91:802-809.

Hart J F and Lisa J R. Histogenesis of Laennec's cirrhosis. New York State Journal of Medicine, 1937;37: 1619-1632.

Hashizume M, Ohta M, Ueno K, Tanoue K, Kitano S, Sugimachi K Endoscopic ligation of oesophageal varices compared with injection sclerotherapy: A prospective randomised trial. Gatrointestinal Endoscopy 1993;39:123-126

Haskal, Z.V., Pentecost, M.J., Soulen, M.C., Schlansky-Goldberg, R.D., Baum, R.A., Cope, C. Transjugular intrahepatic portosystemic shunt stenosis and revision. Early and mid term results. Am J Roentgenol. 1994; 163:439-44

Hauenstein $\mathrm{KH}$, Haag $\mathrm{K}$, Ochs $\mathrm{A}$, Langer $\mathrm{M}$, Rossle $\mathrm{M}$. The reducing stent: Treatment for transjugular intrahepatic portosystemic stent-shunt induced refractory hepatic encephalopathy and liver failure. Radiology. 1995; 194 : $175-179$ 
Haussinger, D., Laubenberger, J., vom Dahl, S., Ernst, T., Bayer, S., Langer, M., Gerok, W., Hennig, J. Proton magnetic resonance spectroscopy studies on human brain myo-inositol in hypo-osmolarity and hepatic encephalopathy. Gastroenterology. 1994;107: 1475-1480.

Hayes P C, Cumming A D, Craig K J, Watson M, Bouchier I A D. (1992) Portal haemodynamics and humoral factors in cirrhosis with and without ascites. American Journal of Gastroenterology, 87: 1433-1438.

Hayes PC, Davis JM, Lewis JA, Bouchier IAD. Meta-analysis of the value of propranolol in the prevention of variceal haemorrhage. Lancet 1990;336:153-156

Helton WS, Belshaw A, Althaus S, Park S, Coldwell D, Johanson K, Critical appraisal of the angiographic portacaval shunt (TIPS). Am. J. Surg. 1993; 165: $566-571$

Henderson JM, Kutner MH, Millikan WJ, Galambos JT, Riepe SP, Brooks WS, Bryan FC et al. Endoscopic variceal sclerosis compared with distal splenorenal shunt to prevent recurrent variceal bleeding in cirrhosis. A prospective randomized controlled trial. Ann Intern Med 1990;112-262-269.

Henriksen J H, Ring-Larsen $\mathrm{H}$, Christensen $\mathrm{N}$ J. Plasma noradrenaline in patients with liver cirrhosis in relation to ascites and treatment. Clinical Physiology (suppl 1): 1981; 66-70.

Henriksen J H, Ring-Larsen H, Christensen N J. Sympathetic nervous activity in cirrhosis. A survey of plasma catecholamine studies. Journal of Hepatology, 1984;1: 55-65.

Henriksen $\mathrm{JH}$, Ring-Larsen $\mathrm{H}$ Hepatorenal disorders: role of the sympathetic nervous system. Seminars in Liver Disease 1994;14:35-43

Henriksen JH, Ring-Larsen $\mathrm{H}$, Christensen NJ. Sympathetic nervous activity in cirrhosis. A survey of plasma catecholamine studies. Journal of Hepatology 1984;1:55-65.

Herredia D, Tores J, Orten N and Rodes J. Lactitol vs lactulose in the treatment of chronic recurrent portal-systemic encephalopathy. J Hepatol. 1988; 7: 106-110.

Herrick F C. An experimental study into the cause of the increased portal pressure in portal cirrhosis. Journal of Experimental Medicine, 1907;9: 93104. 
Hindfelt B, Plum F, Duffy TE. Effect of acute ammonia intoxication on cerebral metabolism in rats with portacaval shunts. J Clin Invest 1977; 59: 386-396.

Hou MC, Lin HC, Kuo BIT, Chen CH, Lee FY, Lee SD Comparison of endoscopic injection sclerotherapy and ligation for the treatment of oesophageal variceal haemorrhage: A prospective randomised trial Hepatology 1995;21:1517-1512.

Hsia HC, Lee FY, Tsai YT, Lee SD, Lai HK, Lin WJ, Lay CS, et al. Comparison of somatostatin and vasopressin in the control of acute esophageal vasopressin hemorrhage. A randomised controlled study. Chin J Gastroenterol 1990;7:71-78.

Huizinga W, Angorn, P, Baker L. Esophageal transection versus injection sclerotherapy in the management of bleeding esophageal varices in patients at high risk. Surg Gynecol Obstet. 1985;160:539-546.

Hunt S, Vaamonde C, Ratassi T. (1979) Circulating levels of vasoactive polypeptide in liver disease. Archives of Internal Medicine, 170: 280-295.

Ideo G, Bellati G, Fesce E, Grimoldi D. Nadolol can prevent the first gastrointestinal bleeding in cirrhotics: A prospective, randomized study. Hepatology 1988; 8:6-9.

Inokuchi K, Sugimachi K, Sato T, Sato H, Isono K, Okuda K, et al. Improved survival after prophylactic portal nondecompression surgery for esophageal varices: A randomized clinical trial. Hepatology 1990; 12:1-6.

Italian Multicenter Project for Propranolol in Prevention of Bleleding. Propranolol prevents first gastrointestinal bleeding in non-ascitic cirrhotic patients. Final report of a multicenter randomized trial. J Hepatol 1989;9:7583.

Ito $\mathrm{M}$, Kikuchi $\mathrm{S}$, Matsumoto $\mathrm{H}$. Glucose metabolism in the rat cerebral cortex in chronic hepatic failure with reference to glucose-derived amino acids. No To Shinkei 1986; 38: 353-357.

Itzhak Y, RoigCantisano A, Dombro RS, Norenberg MD Acute Liver failure and hyperammonemia increase peripheral-type benzodiazepine receptor binding and pregnenolone synthesis in mouse brain. Brain Research 1995; $705: 345-348$

Jackson FC, Perin EB, Smith AG, et al. A clinical investigation of the portacaval shunt: II, survival analysis of the prophylactic operation. Am J Surg. 1968;115:22. 
Jacobs S, Chang R W S, Lee B, Rawaf A Al, Pace N C, Salam I. Prediction of outcome in patients with acute variceal haemorrhage. $\mathrm{Br} J$ Surg 1989;76:123-126.

Jalan R, Elton RA, Redhead DN, Simpson KJ, Finlayson NDC, Hayes PC Analysis of prognostic variables in the prediction of shunt failure, variceal rebleeding, early mortality and encephalopathy following the Transjugular intrahepatic portosystemic stent-shunt (TIPSS) Journal of Hepatology 1995;23:123-128

Jalan R, Forrest EH, Redhead DN, Hayes PC Reduction in renal blood flow with acute increase in portal pressure gradient: Evidence for the existence of hepatorenal reflex in man. Gut 1997;40:664-670

Jalan R, Forrest EH, Stanley AJ, Redhead DN, Forbes J, Dillon JD, MacGilchrist AJ, Finlayson NDC, Hayes PC A randomised trial comparing transjugular intrahepatic portosystemic stent-shunt with variceal band ligation in the prevention of rebleeding from oesophageal varices Hepatology 1997; 26:1115-1122

Jalan R, Harrison DJ, Redhead DN, Hayes PC. Transjugular intrahepatic portosystemic stent - shunt and the role of biliary venous fistula. J Hepatol 1996;24:169-176

Jalan R, John TG, Redhead DN, Finlayson NDC, Garden OJ, Hayes PC. A comparative study of the transjugular intrahepatic portosystemic stent-shunt (TIPSS) and oesophageal transection in uncontrolled variceal haemorrhage. American J. Gastroenterology 1995;11:1932-1936

Jalan R, Simpson KJ, Redhead DN, Chalmers N, Hayes PC. Transjugular intrahepatic portosystemic stent shunt (TIPSS): long term follow-up. Quarterly Journal of Medicine.1994;87:565-73

Jalan R, Turjanski N, Taylor-Robinson SD, Koepp MJ, Richardson MP, Wilson J, Schweiso J, Bryant DJ, Bell JD, Brooks D Pathogenesis of chronic hepatic-encephalopathy (CHE) - A positron emission tomography (PET) with $\mathrm{C}^{11}$ flumazenil and $\mathrm{H}^{1}$ magnetic-resonance spectroscopic (MRS) study Hepatology 1996;24:1298A

Jalan, R, Redhead, DN, Forrest, EH, Hayes, PC. Relationship between directly measured portal pressure and variceal haemorrhage. Am J Gastro 1995; $90: 1994-1996$

Jalan, R., John,T.G., Redhead, D.N., Finlayson,N.D.C., Garden,O.J., Hayes,P.C.. A comparative study of the transjugular intrahepatic portosystemic stent-shunt (TIPSS) and oesophageal transection in 
Jalan, R., Plevris, J.N., Jalan, A.R., Finlayson, N.D.C., Hayes, P.C. A pilot study of ICG clearance in the prediction of graft function following orthotopic liver transplantation. Transplantation. 1994; 58: 196-200.

Jaramillo JL, de la Mata M, Mino G, Costan G, Gomez-Camacho F. Somatostatin versus Sengstaken balloon tamponade for primary haemostasis of bleeding esophageal varices. J Hepatol 1991;12:100-105.

Jenkins SA, Baxter JN, Corbett W, et al. A prospective randomised controlled clinical trial comparing somatostatin and vasopressin in controlling acute variceal haemorrhage. British Medical Journal 1985; 290:275-278.

Jenkins SA, Copeland G, Kingsnorth A, Shields R. A prospective randomized controlled trial comparing somatostatin (SMS) and injection sclerotherapy in the control of acute variceal haemorrhage: an interim report. (Abstract). Gut 1992;33:F221.

Jones EA, Gammal SH, Martin P. Hepatic encephalopathy: new light on an old problem. Quarterly J Med 1988; 69: 851-867.

Jones EA, Schafer DF. Fulminant hepatic failure. In Hepatology, A Textbook of Liver Diseases. ed. by D.Zakim and T.D.Boyer, pp 460-492. WB Saunders Co. Philadelphia, 1990.

Keil J W, Pitts V, Benoit J N, Granger D N, Shepherd A P. Reduced vascular sensitivity to norepinephrine in portal hypertensive rats. American Journal of Physiology, 1985;248: G192-G195.

Kelty $\mathrm{R} \mathrm{H}$, Baggentoss $\mathrm{A} \mathrm{H}$ and Butt $\mathrm{H}$ R. The relation of the regenerated liver nodule to the vascular bed in cirrhosis. Gastroenterology, 1950;15: 285-296.

Kircheis G, Nilius R, Held C, Berndt H, Buvhner M, Gortelmeyer R, Hendricks R, Kruger B, Kuklinski B, Meister H, Otto HJ, Rink C, Rosch W, Stauch S. Therapeutic efficacy of L-ornithine-L-aspartate infusions in patients with cirrhosis and hepatic encephalopathy: Results of a placebo-controlled, double-blind study. Hepatology 1997;25:1351-1360

Kitano S, Terblanche J, Kahn D et al. Venous anatomy of the lower oesophagus in portal hypertension: practical implications. British Journal of Surgery, 1986;73: 525-531. 
Kobe E, Zipprich B, Schentke KU, Nilius R. Prophylactic endoscopic sclerotherapy of esophageal varices - A prospective randomized trial. Endoscopy $1990 ; 22: 245-248$.

Koch $\mathrm{H}$, Henning $\mathrm{H}$, Grimm $\mathrm{H}$, Soehendra N. Prophylactic sclerosing of esophageal varices - Results of a prospective controlled study. Endoscopy $1986 ; 18: 40-43$.

Korula J, Balart LA, Radvan G, Zweiban BE, Larson AW, Kao HW, Yamada S. A prospective randomized controlled trial of chronic esophageal variceal sclerotherapy. Hepatology 1985;5:584-589.

Korula J, Yellin A, Yamada S, Weiner J, Cohen H, Reynolds TB. A prospective randomized controlled comparison of chronic endoscopic variceal sclerotherapy and portalsystemic shunt for variceal hemorrhage in Child Class A cirrhotics. (Abstract). Hepatology 1988;8:1242.

Kravetz D, Bosch J, Teres J, et al. Comparison of intravenous somatostatin and vasopressin infusions in treatment of acute variceal hemorrhage. Hepatology $1984 ; 4: 442-446$.

Kreis, R, Farrow, N, and Ross, BD Localised ${ }^{1} \mathrm{H}$ NMR spectroscopy in patients with chronic hepatic encephalopathy. Analysis of changes in cerebral glutamine, choline and inositols. NMR Biomed. 1991; 4: 109-116.

La Berge JM, Ring EJ, Gordon RL, Lake JR, Doherty MM, Somberg KA, Roberts JP, Ascher NL Creation of a transjugular intrahepatic portasystemic shunt with Wallstent endoprosthesis:Results in 100 patients. Radiology 1993; $187: 413-20$

LaBerge JM, Ferrell LD, Ring, EJ, Gordon RL. Histopathological study of stenotic and occluded transjugular intrahepatic portosystemic shunt. J Vasc Intervent Radiol 1993; 4: 779-86

Lacy, A.C., Navasa, M., Gilabert, R., Bru,C., Garcia-Pagan, J.C., GarciaValdecasas, J.C., Grande, L. Long-term effects of distal splenorenal shunt on hepatic haemodynamics and liver function in patients with cirrhosis: Importance of reversal of portal blood flow. Hepatology 1992; 15:616-622

Laffi G, Marra F, Failli P, Ruggiero M, Cecchi E et al. Defective signal transduction in platelets from cirrhotics is associated with increased cyclic nucleotides. Gastroenterology 1993;105: 148-156.

Laine L, El-Newihi HM, Migikovsky B, Sloane R, Garcia F. Endoscopic ligation compared with sclerotherapy for the treatment of bleeding esophageal varices. Ann Intern Med 1993;119:1-7. 
Laine L, Stein C, Sharma V Randomised comparison of ligation versus ligation plus sclerotherapy in patients with bleeding oesophageal varices. Gastroenterology 1996;110:529-533

Laine L. Ligation: Endoscopic treatment of choice for patients with bleeding oesophageal varices. Hepatology 1995;22:661-665

Lang F, Tschernko E, Schulze E, Ottl I, Ritter M, Volkl H, Hallbrucker C, Haussinger $D$ Hepatorenal reflex regulating kidney function Hepatology 1991;14:590-594

Langer B, Taylor BR, MacKenzie DR, et al. Further report of a prospective randomized trial comparing distal spleno-renal shunt with end to side portacaval shunt. Gastroenterology 1985;88:424-429.

Lapointe D S, Olson M S. Alteration of hepatic tissue spaces by platelet activating factor and phenylephrine. Hepatology 1989; 9: 278-284.

Larson AW, Cohen H, Zwieiban Ba, Chapman D, Gourdji M, Korula J, Weiner

D. Acute esophageal variceal sclerotherapy. JAMA 1986;255:497-500.

Laubenberger J, Haussinger D, Bayer S, Gufler H, Kennig J, Langer M Proton magnetic resonance spectroscopy of the brain in symptomatic and asymptomatic patients with liver cirrhosis Gastroenterology 1996;112:16101616

Le Moine O, Adler M, Bourgeois N, Delhaye M, Deviere J, Gelin, M. Vandermeeren A, Van Gossum A, Vereerstraeten A, Vereerstraeten $P$, Cremer M. Factors related to early mortality in cirrhotic patients bleeding from varices and treated by urgent sclerotherapy. Gut 1992;33:1381-1385.

Lebrec D, De Fleury P, Rueff B, et al. Portal hypertension, size of esophageal varices, and risk of gastrointestinal bleeding in alcoholic cirrhosis. Gastroenterology 1980; 79:1139-1144.

Lebrec D, Giuily N, Hadengue A, Vilgrain V, Moreau R, Poynard T, Gadano A, Lassen C, Benhamou JP, Erlinger S. Transjugular intrahepatic portosystemic shunts: Comparison with paracentesis in patients with cirrhosis and refractory ascites: A randomized trial Journal of Hepatology, $1996 ; 25: 135-144$

Lebrec D, Poynard T, Bernuau J, Bercoff E, Nouel O, Capron JP, Poupon R et al. A randomized controlled study of propranolol for prevention of recurrent gastrointestinal bleeding in patients with cirrhosis: a final report. Hepatology 1984;4:355-358. 
Lebrec D, Poynard T, Capron JP, Hillon P, Geoffrey P, Roulot D, Chaput JC, Rueff B, Benhamou JP. Nadolol for prophylaxis of gastrointestinal bleeding in patients with cirrhosis. A randomised trial. J Hepatol 1988;7:118-125

Lebrec D, Poynard T, Hillon P, Benhamou JP. Propranolol for prevention of recurrent gastrointestinal bleeding in patients with cirrhosis. A controlled study. N Eng J Med 1981; 305:1371-1374.

LeBrec, D., Giuily, N., Hadengue, A., Vilgrain, V., Lassen, C., Poynard, T., et.al. Transjugular intrahepatic portosystemic shunt (TIPS) vs Paracentesis for refractory ascites. Results of a randomised trial. Hepatology. 1994; 20: 417. (abstract)

Lee F Y, Colombato L A, Albillos A, Groszmann R J. N-nitro-L-arginine administration corrects peripheral vasodilatation and systemic capillary hypotension and ameliorates plasma volume expansion and sodium retention in portal hypertensive rats. Hepatology, 1993; 17: 84-90.

Lee S S, Chilton E L, Pak J M. Adenosine receptor blockade reduces splanchnic hyperaemia in cirrhotic rats. Hepatology, 1992;15: 1107-1111.

Lee S S, Hadengue A, Moreau R et al. Naloxone does not alter haemodynamics in cirrhosis: studies in humans and rats. Journal of Hepatology, 1987;5: 149-153.

Lee S S, Moreau R, Hadengue A, Cerini R, Koshy A, Lebrec D. Glucagon selectively increases splanchnic blood flow in patients with well compensated cirrhosis. Hepatology, 1988;8: 1501-1505.

Lee YF, Tsai YT, Laid KH, Lay CS, Chang TT, Malik U, Lin CY, et al. A randomised controlled study of triglycyl-vasopressin and vasopressin plus nitroglycerin in the control of acute esophageal variceal hemorrhage. Chin J Gastroenterol 1988;5:131-138.

Lee FY, Colombata LA, Albillos A, Groszmann RJ. N-nitro-L-arginine administration corrects peripheral vasodilatation and systemic capillary hypotension and ameliorates plasma volume expansion and sodium retention in portal hypertensive rats. Hepatology 1993;17:84-90

Leevy C M, Ten Hove W, Opper A and Popovic S. Influence of ethanol and microsomal drugs on hepatic haemodynamics. Annals of the New York Academy of Science, 1970;170: 315-330.

Leiberman F L, Denison E K, Reynolds T B. The relationship of plasma volume, portal hypertension, ascites, and renal sodium and water retention in cirrhosis: the overflow theory of ascites formation. Ann NY Acad Sci. 1970 
Levacher S, Blaise M, Paterson D, Letoumelin Ph, Lepandry C, Pourriat JL. Early administration of terlipressin plus transdermal nitroglycerin of active upper gastrointestinal hemorrhage in cirrhotic patients (Abstract). J Hepatol 1994;21:S46.

Lind, C.D., Malisch, T.W., Chong, W.K., Richards, W.O., Pinson, C.W., Meranze, S.G., Mazer, M. Incidence of shunt occlusion or stenosis following transjugular intrahepatic portosystemic shunt placement. Gastroenterology 1994;106:1277-1283

Linssen ,A.C.G., Van Dam, F.S.A.M., Engelsman, E., Van Benthem, J., Hanewald, G.J.F.P.Leven met cytostatica. Pharmaceutisch. Weekblad. 1979; 114: 501

Liu JD, Jeng YS, Chen PH, Siauw CP, Ko FT, Lin KY. Endoscopic injection sclerotherapy and propranolol in the prevention of recurrent variceal bleeding. 1990 Gastroenterology World Congress abstract book; GP 1181.

Llach, J., Gines, P., Arroyo, V., Salmeron, J.M., Gines, A., Jiminez, W., Gaya, J., Rivera, F., Rodes, J. Effect of dipyridamole on the kidney function in cirrhosis. Hepatology. 1993; 17:59-64.

Llach J, Gines P, Arroyo V, et.al. Prognostic value of arterial pressure, endogenous vasoactive systems, and renal function in cirrhotic patients admitted to the hospital for the treatment of ascites. Gastroenterology $1988 ; 94: 482-487$

Lo GH, Lai KH, Cheng JS, Hwu CH, Chang CF, Chang SM, Chiang HU. A prospective, randomised trial of sclerotherapy versus ligation in the management of bleeding oesophageal varices. Hepatology 1995;22:466-471

Lockwood $\mathrm{AH}$, Yap EWH, and Wong W-H. Cerebral ammonia metabolism in patients with severe liver disease and minimal hepatic encephaloapthy. J Cerebr Blood Flow Metab 1991; 11: 337-341.

Longo JM, Bilbao JI, Rousseau HP, et.al. Transjugular intrahepatic portosystemic shunt. Evaluation with Doppler sonography. Radiology 1993:186:529-534

Lotterer, E., Moosmuller, A., Wengert, A., Hahn, E.G., Fleig, W.F. Effects of transjugular intrahepatic portal systemic shunt on liver function in patients with alcoholic cirrhosis. Hepatology. 1994; 20:47 (abstract)

Luyten, P.R., den Hollander, J.A., Bovée, W.M.M.J., Ross B.D., Bosman, D.K., and Chamuleau, R.A.F.M. ${ }^{31} \mathrm{P}$ and ${ }^{1} \mathrm{H}$ NMR spectroscopy of the human brain in chronic hepatic encephalopathy. Proc 8th Ann Mtg Soc Magn Reson Med. 1989;1: 375. 
MacGilchrist A J, Deighton N M, Hamilton C A, Reid J L. Binding studies of platelet alpha 2- and lymphocyte beta 2- adrenoceptors in patients with cirrhosis. British Journal of Clinical Pharmacology, 1990;30: 644-647.

MacGilchrist A J, Susmner D, Reid J L. Impaired pressor reactivity in cirrhosis: evidence for a peripheral vascular defect. Hepatology 1991;13: 689-694.

MacGilchrist AJ, Craig $K$ J, Hayes $P$ C, Cumming A D. Effect of serine protease inhibitor, aprotinin, on systemic haemodynamics and renal function in patients with hepatic cirrhosis and ascites. Clinical Science, 1994;87: 329-335.

MacMathuna P, Vlavianos $P$, Wendon J, Westaby D, Williams R. Role of adenosine in the haemodynamic disturbances of cirrhosis and portal hypertension. Hepatology, 1990b;12: 852.

MacMathuna $P$, Vlavianos $P$, Westaby $D$ and Williams $R$. Taking the tension out of the portal system. Scandinavian Journal of Gastroenterology, 1990a;175: 131-145.

Malinchoc $M$, Kamath $P$, Peine $C$ et.al. A revised model for predicting survival in patients undergoing elective transjugular intrahepatic portosystemic shunts (TIPS). Gastroenterology 1996;110:A1255

Mallory A, Schaefer J W, Cohen J R, Holt AS, Norton LW. Selective intraarterial vasopressin infusion for upper gastrointestinal tract hemorrhage. $A$ controlled trial. Arch Surg 1980;115:30-32.

Mans AM, Biebuyck JF, Shelley K, Hawkins RA. Regional blood brain barrier permeability to amino acids after portacaval anastomosis. J Neurochem 1982;38:705-13

Marteau $\mathrm{P}$, Ballet $\mathrm{F}$, Chaxouilleres $\mathrm{O}$ et al. Effect of vasodilators on hepatic microcirculation in cirrhosis: a study in the isolated perfused rat liver. Hepatology, 1989; 9: 820-823.

Martin T, Taupignon A, Lavignolle A, Perrin D, LeBodic L. Pr $\square$ vention des r $\square$ cidives $h \square$ morragiques chez des malades atteints de cirrhose. R $\square$ sultats d'une $\square$ tude control $\square$ comparant propranolol et scl $\square$ rose endoscopique. Gastroenterol Clin Biol 1991;15:833-837.

Martin, M., Zajko, A.B., Orons, P.D., Dodd, G., Wright, H., Colangelo, J., Tartar, R., Transjugular intrahepatic portosystemic shunt in the management of variceal bleeding: indications and clinical results. Surgery 1993;114:719727 
Matthews, J.N.S., Altman, D.G., Campbell, M.J., Royston; P. Analysis of serial measurements in medical research. BMJ 1990;300:230-35

Mc Corkle, R., Young, K. Development of a symptom distress scale. Cancer Nursing. 1978; 373

McClain C $\mathrm{J}$ and Cohen D A. Increased tumour necrosis factor (TNF) production by monocytes in alcoholic hepatitis. Hepatology, 1989;9: 349351.

McCormick PA, Dick R, Panagou EB, Chin JKT, Greenslade L, Burroughs AK Emergency transjugular intrahepatic portosystemic stent-shunt salvage treatment for uncontrolled variceal bleeding. British J Surgery 1994;81:1324-1327.

McCormick PA, Mistry P, Kaye G, Burroughs AK, Mclntyre N Intravenous albumin infusion is an effective therapy for hyponatraemia in cirrhotic patients with ascites. Gut 1990;31:204-207

McIndoe A H. Vascular lesions of portal cirrhosis. Archives of Pathology, 1928;5: 23-40.

McKee R. A study of octreotide in oesophageal varices. Digestion 1990;45:60-65.

Merigan TC, Plotkin GR, Davidson CS. Effect of intravenously administered posterior pituitary extract on hemorrhage from bleeding esophageal varices. N Engl J Med 1962;266:134-135.

Merkel C, Bolognesi M, Bellon S, Zuin R, Noventa F, Finucci G, et al. Prognostic usefulness of hepatic vein catheterization in patients with cirrhosis and esophageal varices. Gastroenterology 1992; 102:973-979.

Merli M, Riggio O, Capocaccia L, Ziparo V, Bolognese A, Rossi P, Salerno F, DeFranchis R, Fiaccadori $F$, and GIST. Transjugular intrahepatic portosystemic shunt (TIPS) vs Endoscopic sclerotherapy in preventing variceal rebleeding. Preliminary results of a randomised controlled trial. Hepatology. 1994; 20: 107A (abstract)

Michael MJ. The pathology of hepatolienal fibrosis. Journal of Pathology and Bacteriology, 1934;39: 481-582.

Michalak A, Rose C, Butterworth J, Butterworth RF Neuroactive amino acids and glutamate (NMDA) receptors in frontal cortex of rats with experimental acute liver failure. Hepatology 1996; 24:908-913 
Mikkelson W P, Edmondson $\mathrm{H} \mathrm{A}$, Peters $\mathrm{R} L$ et al. Extra and intrahepatic portal hypertension without cirrhosis. Annals of Surgery, 1965;162: 602620.

Millikan WJ, Warren WD, Henderson JM, Smith RB, Salam AA, Galambos JT, Kutner $\mathrm{MH}$ et al. The Emory prospective randomized trial: selective versus non-selective shunt to control variceal bleeding. Ann Surg 1985;201:712-721.

Minuk G Y, MacCannell $\mathrm{K} \mathrm{L}$. Is the hypotension of cirrhosis a GABAmediated process? Hepatology, 1988;8: 73-77.

Moller S, Emmeluth $\mathrm{C}$, Henriksen $\mathrm{J} \mathrm{H}$. Elevated circulating plasma endothelin-1 concentrations in cirrhosis. Journal of Hepatology, 1993;19: 285-290.

Moncada S, Higgs A. The L-arginine-nitric oxide pathway. $\mathrm{N}$ Eng J Med, 1993; 329: 2002-2012.

Moore KP, Taylor G W, Maltby N, Dollery C T D, Williams R. Increased production of cysteinyl leukotrienes in hepatorenal syndrome. Journal of Hepatology 1990;11:262-271.

Moore KP, Ward P, Taylor G, Williams R. Systemic and renal production of thromboxane $A_{2}$ and prostacyclin in decompensated liver disease and hepatorenal syndrome. Gastroenterology 1991;100:1069-1077

Moore KP. The hepatorenal syndrome Clinical Science 1997;92:433-443

Moorey B, Greer S, Watson M, Gorman c, Rowden L. The development and validation of a test battery for detecting and monitoring everyday memory problems. J Clin Exp Neuropsychol 1989;6:855-870

Moorey, S., et.al.The factor structure and factor stability of the HAD Scale in patients with cancer. British Journal of Psychiatry. 1991; 158:255-259

Morali, G.A., Floras, J.S., Legault, L., Tobe, S., Skorecki, K.L., Blendis, L.M. Muscle sympathetic nerve activity and renal responsiveness to atrial natriuretic factor during the development of ascites. Am J.Med. 1991;91:383392

Moreau R, Cailmail R, Gaudin C, Hamon G, Lebrec D. Renal and hemodynamic responses to a novel inhibitor of vasopressin secretion, RU 51599, in rats with cirrhosis. Journal of Hepatology, 1994;21: S7. 
Moreau, R., Gaudin, C., Hadengue, A., Braillon, A., Roulot, D., Bacq, Y., et.al. Renal haemodynamics in patients with cirrhosis: relationship with ascites and renal failure. Nephron. 1993;65:359-363

Moreto $\mathrm{M}$, Zaballa $\mathrm{M}$, Bernal $\mathrm{A}$, et al. A randomized trial of tamponade or sclerotherapy as immediate treatment for bleeding esophageal varices. Surg Gynecol Obstet. 1988;167:331-334.

Morgan MY and Hawley KE. Lactitol vs lactulose in the treatment of acute hepatic encephalopathy in cirrhotic patients: a double-blind, randomized trial. Hepatology. 1987; 7 : 1278-1284.

Moritoki $H$, Matsugi $T$, Takase $H$, Ueda $H$, Tanioka A. Evidence for the involvement of the cyclis GMP in adenosine-induced, age-dependant vasodilatation. British Journal of Pharmacology, 1990;100: 569-575.

Moriyasu F, Nishida O, Ban N, et.al. 'Congestion index' of the portal vein. AJR 1986:146:735-739

Mullen KD, Jones EA. Natural benzodiazepines and hepatic encephalopathy. Seminars in Liver Diseases 1996;16:255-264

Murad F. Cyclic guanosine monophosphate (cGMP) as a mediator of vasodilatation. Journal of Clinical Investigation, 1986;78: 1-5.

Mutchnik M G, Lernoz E, Conar H O. Effect of portocaval anastomosis on hypersplenism. Dig Dis Sci, 1980;25: 929-938.

Navasa $\mathrm{M}$, Bosch $\mathrm{J}$, Reichen $\mathrm{J}$ et al. Effects of verapamil on hepatic and systemic haemodynamics and liver function in patients with cirrhosis and portal hypertension. Hepatology 1988;8: 850-854.

Navasa M, Chesta J, Bosch J, Rodes J. Reduction of portal pressure by isosorbide - 5 - mononitrate in patients with cirrhosis. Effects upon splanchnic and systemic haemodynaimcs and liver function. Gastroenterology 1989;96:1110-1118

Nishida O. Moriyasu F. Nakamura T. Ban N. Tamada T. Kawasaki T. Uchino H.Relationship between splenic and superior mesenteric venous circulation. Gastroenterology. 1990; 98:721-725

North Italian Endoscopic Club (NIEC) for the study and treatment of esophageal varices. Prediction of first variceal haemorrhage in patients with cirrhosis of the liver and esophageal varices. a prospective multicenter study. N Eng J Med 1988, 319:983-989 
O'Carrol RE, Hayes PC, Ebemeir KP, Dougall N, Murray C, Best JJK, Bouchier IAD, Goodwin GM Regional cerebral blood flow and cognitive function in patients with chronic liver disease. Lancet 1991;337:1250-1253

Oberti F, Sogi P, Cailmail S, Moreau R, Pipy B, Lebrec D. Role of prostacyclin in haemodynamic alterations in conscious rats with extrahepatic or intrahepatic portal hypertension. Hepatology, 1993;18: 621-627.

Ochs A, Rossle M, Haag K, Hauenstein KH, Deibert $P$, Siegerstetter V, Huonker M, Langer M, Blum HE The transjugular intrahepatic portal-systemic shunt procedure for refractory ascites. New Eng J Med 1995;332:1192-1197

Ohkibudo H, Okuda K, lida S, Ohnishi K et al. Role of portal and splenic vein shunts and impaired hepatic extraction in the elevated serum bile acids in liver cirrhosis. Gastroenterology, 1984;86: 514-520.

Orrego $\mathrm{H}$, Blendis L M, Crossley I R et al. Correlation of intrahepatic pressure with collagen in the Disse space and hepatomegaly in humans and in the rat. Gastroenterology, 1981;80: 546-556.

Orrego $\mathrm{H}$, Medline $\mathrm{A}$, Blendis $\mathrm{L} M$ et al. Collagenisation of the Disse space in alcoholic liver disease. Gut, 1979;20: 673-679.

Owen, A.M., Roberts, A.C., Polkey, C.E., Sahakian, B.J., Robbins, T.W. Extra dimensional versus intra-dimensional set shifting performance following frontal lobe excisions, temporal lobe excisions ar amygdalohippocampectomy in man. Neuropsychologia. 1991; 29: 991-1006

Palmaz J C, Sibbitt R R, Reuter S R, Tio F O, Rice W J. Expandable intraluminal graft: a preliminary study. Radiology 1985;156:73-77.

Palmaz,J., Garcia,F., Sibbit,R.R., Tio,F.O., Kopp,D.T., Schwesinger,W., Lancaster,J.L., et.al. Expandable intrahepatic portasystemic shunt stents in dogs with chronic portal hypertension. Am.J.Roengenol. 1986;147:1251-54

Palmer ED, Brick IB. Correlation between severity of esophageal varices in portal cirrhosis and their propensity toward hemorrhage. Gastroenterology 1956;30:85-90.

Panes J, Teres J, Bosch J, et al. Efficacy of balloon tamponade in the treatment of bleeding gastric and esophageal varices: results in 151 consecutive episodes. Dig Dis Sci.. 1988;33:454-459.

Paquet KJ, Feusener $\mathrm{H}$. Endoscopic sclerosis and esophageal balloon tamponade in acute hemorrhage from esophagogastric varices: a prospective randomised trial. Hepatology 1985;5:580-583 
Paquet $\mathrm{KJ}$, Koussouris $\mathrm{P}$. Is there an indication of prophylactic endoscopic paravariceal injection sclerotherapy in patients with liver cirrhosis and portal hypertension? Endoscopy 1986; 18:32-35.

Parsons-Smith BC, Sumerskill WHJ, Dawson AM. The electroencephalograph in liver disease. Lancet. 1957; 2: 867-871

Pascal JP, Cales P \& Multicentre Study Group. Propranolol in the prevention of first upper gastrointestinal tract haemorrhage in patients with cirrhosis of the liver and oesophageal varices. N Engl J Med 1987;317:856-861.

Perarnau, J.M., Raabe, J.J., Schwing, D., Rucin, B., Monchovet, S., Rossle, M., Arbogast, J. Anastomose porto-cava intra hepatique par voie transjugulaire. Gastroenterol Clin Biol 1993;17:422-430

Perez-Ayuso R, Arryoy V, Camps J, Rimola A, Costa J, Gaya J, Rivera F, Rodes $\mathrm{J}$. Renal kallikrein excretion in cirrhotics with ascites; relationship to renal haemodynamics. Hepatology, 1984;4: 247-252.

Peroutka S J. Receptor 'families' for 5-hydroxytryptamine. Journal of Cardiovascular Pharmacology, 1990;16 (suppl 3): S8-S14.

Persson PB, Ehmke H, Kircheim HR Sympathetic modulation of renal autoregulation by carotid occlusion in concious dogs. Am J Physiol 1990;258:F364-70

Piai G, Cipolletta L, Claar M, Marone G, Bianco MA, Forte G, et al. Prophylactic sclerotherapy of high-risk esophageal varices: Results of a multicentric prospective controlled trial. Hepatology 1988; 8:1495-1500.

Pizcueta M P, Casamitjana R, Bosch J, Rodes J. Decreased systemic vascular sensitivity to norepinephrine in portal hypertensive rats: role of hyperglucagonism. American Journal of Physiology, 1990;258: G191G195.

Pizcueta M P, Pique J M, Bosch J, Whittle B J R et al. Effects of inhibiting nitric oxide biosysnthesis on the systemic and splanchnic circulation of rats with portal hypertension. British Journal of Pharmacology, 1992A;105: 184190.

Pizcueta M P, Pique J M, Fernandez M, Bosch J et al. Modulation of the hyperdynamic circulation of cirrhotic rats by nitric oxide inhibition. Gastroenterology, 1992B;103: 1909-1915.

Planas R, Boix J, Broggi M, Cabre E, GomesVieira MC, Morillas R, et al. Portacaval shunt versus endoscopic sclerotherapy in the elective treatment of variceal hemorrhage. Gastroenterology 1991; 100:1078-1086. 
Planas R, Gines P, Arroyo V, Llach J, Panes J, Vargas V, Salmeron JM, Gines A, Toledo C, Rimola A, Jimenez W, Asbert M, Gassull MA, Rodes J Dextran-70 vs albumin as plasma expanders in cirrhotic patients with tense ascites treated with total paracentesis. Results of a randomised study. Gastroenterology 1990;99:1736-1744

Planas R, Gomes-Vieira M C, Cabre E, Armengol M, Quer J C, Boix J, Morillas R, Abad-Lacruz A, Broggi M, Gassull M A. Prognostic Factors of hepatic encephalopathy after portacaval anastomosis: A multivariate analysis of 50 patients. Am J Gastroenterol 1992;87:1792-1796.

Planas R, Quer JC, Boix J, Canet J, Armengol M, Cabre E, et al. A prospective randomized trial comparing somatostatin and sclerotherapy in the treatment of acute variceal bleeding. Hepatology 1994; 20:370-375.

Polio J, Groszmann RJ. Hemodynamic factors involved in the development and rupture of esophageal varices: A pathophysiologic approach to treatment. Seminars in Liver Disease 1986; 6:318-331.

Pomier-Layrargues G., Shapcott D., Spahr L., and Butterworth R.F. Accumulation of manganese and copper in the pallidum of cirrhotic patients: role in the pathogenesis of hepatic encephalopathy? Metab Brain Dis. 1995; 10: $351-354$

Popper H, Elias $\mathrm{H}$ and Petty D E. Vascular patterns of the cirrhotic liver. American Journal of Clinical Pathology, 1952;22: 717-732.

Popper H, Paronetto F, Schaffner F and Perez V. Studies on hepatic fibrosis. Laboratory Investigation, 1961;10: 265-278.

Potzi R, Bauer P, Reichel W, Kerstan E, Renner F, Gangl A. Prophylactic endoscopic sclerotherapy of oesophageal varices in liver cirrhosis. A multicentre prospective controlled randomised trial in Vienna. Gut 1989; 30:873-879.

Power W. Contributions to pathology. Maryland Medical and Surgical Journal, 1: 1840; 306-318. Cited by Child 1954.

Prada A, Bortoli A, Minoli G, Carnovali M, Colombo E, Sangiovanni A. Prediction of oesophageal variceal bleeding: Evaluation of the beppu and North Italian Endoscopic Club scores by an independent group. European Journal of Gastroenterology and Hepatology 1994; 6:1009-1013

Preble R B. Conclusions based on sixty cases of fatal gastrointestinal haemorrhage due to cirrhosis of the liver. American Journal of Medical Science, 1900; 119: 263-279. 
Pruyn, J.F.A., Van Den Heuvel, W.J.A., Jonkers, R.Verantvoording van de klachtenlijst voor kankerpatienten. Studiecentrum Sociale Oncologie Rotterdam. 1980.

Pugh RMN, Murray-Lyon IM, Dawson JL, et.al. Transection of the oesophagus for bleeding oesophageal varices. British Journal of Surgery 1973;60:646-649

Quero JC, Schalm SW Subclinical Hepatic Encephalopathy. Seminars in Liver Disease 1996;16:343-328

Queuniet AM, Czernichow P, Lerebours E, Ducrotte P, Tranvouez JL, Colin R. Etude control $\square$ e due propranolol dans la prev $\square$ ntion des r $\square$ cidives h $\square$ morragiques chez les patients cirrhotiques. Gastroenterol Clin Biol $1987 ; 11: 41-47$.

Quiroga, J., Sangro, B., Nunez, M., Bilbao, I., Longo, J., Garcia-Villarreal, L. Transjugular intrahepatic portal systemic shunt in the treatment of refractory ascites: Effect on clinical, renal, humoral and haemodynamic parameters. Hepatology. 1995;21:986-994

Ramond MJ, Valla D, Gotlib JP, et al. Obturation endoscopique des varices oeso-gastriques par le Bucrylate. Gastroenterol Clin Biol. 1986;10:575-579.

Reich $M$ et.al. Experimental cryoprobe production of intrahepatic portosystemic shunt. J Surg Res 1977;73:14

Reichen $\mathrm{J}$ and Le M. Verapamil favourably influences hepatic microvascular exchange and function in rate with cirrhosis of the liver. Journal of Clinical Investigation, 1986;78: 448-455.

Reichen J. Liver function and pharmacological considerations in pathogenesis and treatment of portal hypertension. Hepatology;1990;11:1066-1078

Reichle FA, Fahmy WF, Golsorkhi M. Prospective comparative clinical trial with distal splenorenal and mesocaval shunts. Am J Surg 1979;137:13-21.

Resnick RH, Chalmers TC, Ishihara AM, Garceau J, Callow AD, Schimmel EM, O'Hara ET et al. A controlled trial of the prophylactic portacaval shunt. A final report. Ann Intern Med 1969;70:675-688.

Resnick RH, Iber FL, Ishiara A, Chalmers TC, Zimmermann H, The Boston Inter-Hospital Liver Group. A controlled study of the therapeutic portacaval shunt. Gastroenterology 1974;67:843-857. 
Reynolds T B, Geller H M, Kuzma O T and Redeker A G. Spontaneous decrease in portal pressure with clinical improvement in cirrhosis. New England Journal of Medicine, 1960;263: 734-739.

Reynolds TB, Donovan AJ, Mikkelsen WP, Redeker AJ, Turril FL, Weiner JM. Results of a 12 year randomized trial of portacaval shunt in patients with alcoholic liver disease and bleeding varices. Gastroenterology 1981;80:10051011.

Richardson P D I and Withrington P G. Liver blood flow (ii). Effects of drugs and hormones on liver blood flow. Gastroenterology, 1981a;81: 356-375.

Richardson $P D I$, Withrington $P G$. The inhibition by glucagon of the vasoconstrictor actions of noradrenaline, angiotensin, and vasopressin on the hepatic arterial vascular bed of the dog. British Journal of Pharmacology, 1976;57: 93-102.

Richardson $\mathrm{PDI}$ and Withrington PG. Liver blood flow (i). Intrinsic and nervous control of liver blood flow. Gastroenterology, 1981b; 81: 159-173.

Richter,G.M., Noeldge,G.,Palmaz,J.C. The transjugular intrahepatic portasystemic stent-shunt(TIPSS): experience results of a pilot study. Cardiovasc. Intervent. Radiol. 1990;13:200-207

Rikkers LF, Burnett DA, Valentine GD, Buchi KN, Cormier RA. Shunt surgery versus endoscopic sclerotherapy for long term treatment of variceal bleeding. Early results of a randomized trial. Ann Surg 1987;206:261-269.

Rimola A, Gines P, Arroyo V et al. Urinary excretion of 6-keto-prostaglandin $\mathrm{FI}$, thromboxane B2, and prostaglandin E2 in cirrhosis with ascites; relationship to functional renal failure (hepatorenal syndrome). Journal of Hepatology, 1986;3: 111-117.

Ring E.J., Lake, J.R., Roberts, J.P., Gordon, R.L., LaBerge, J.M., Read, A.E., et.al. Using transjugular intrahepatic portosystemic shunts to control variceal bleeding before liver transplantation. Ann. Int. Med. 1992; 116:304-309

Ring-Larsen $\mathrm{H}$, Henrikson $\mathrm{J} \mathrm{H}$. Pathogenesis of ascites formation and hepatorenal syndrome: humoral and haemodynamic factors. Semin Liv Dis, 1986;6: 341-352.

Ring-Larsen $\mathrm{H}$, Hesse B, Henriksen JH Christensen NJ. Sympathetic nervous activity and renal and systemic haemodynamics in cirrhosis - plasma norepinephrine concentration, hepatic extraction and renal release. Hepatology 1982;2:304-310. 
Rockey D The cellular pathogenesis of portal hypertension: Stellate cell contractility, endothelin, and nitric oxide Hepatology, 1997;25:2-5

Rodriguez-Moreno F, Santolaria F, Glez-Reimers E, Gomez JL, Batista N, Conde A, Martinez $A$, et al. A randomized trial of somatostatin vs vasopressin plus nitroglycerin in the treatment of acute vasopressin bleeding. (Abstract). J Hepatol 1991;13:S162.

Rosch J, Hanafee W N, Snow H. Transjugular portal venography and radiologic portacaval shunt: an experimental study. Radiology 1969;92:11121114.

Rosch J. Uchida BT. Putnam JS. et al. Experimental intrahepatic portacaval anastomosis: Use of expandable Gianturco stents. Radiology $1987 ; 162: 481-485$.

Rosch,J, Hanafee W N,Snow,H. Transjugular intrahepatic portacaval shunt:An experimental work. Am J Surg 1971;121:588-92

Rose'Meyer R B, Hope W. Evidence that A2-purinoceptors are involved in endothelium-dependant relaxation of the rat thoracic aorta. British Journal of Pharmacology 1990;100: 576-580.

Rossi V, Cales P, Pascal B, Charneau J, Person B, Pujol P, Valentin S et al. Prevention of recurrent variceal bleeding in alcoholic cirrhotic patients: a prospective controlled trial of propranolol and sclerotherapy. J Hepatol $1991 ; 12: 283-289$.

Rossle M, Deibert P, Haag K, Ochs A, Olschewski M, Siegerstetter V, Hauenstein KH, Geiger R, Stiepak C, Keller W, Blum HE Randomised trial of transjugular-intrahepatic-portosystemic shunt versus endoscopy plus propranolol for prevention of variceal rebleeding Lancet 1997;349:1043-1049

Rossle M, Haag K, Ochs A, Sellinger M, Noldge G, Perarnau JM, Berger E, Blum U, Gabelmann A, Hauenstein K, Langer M, Gerok W. The transjugular intrahepatic stent-shunt procedure for variceal haemorrhage. $\mathrm{N}$ Eng J Med 1994; 3: 165-72

Rousselot $\mathrm{L} M$. The role of congestion (portal hypertension) in so-called Banti's syndrome. JAMA 1936;107: 1788-1793.

Rozga J. Jeppsson B. Hagerstrand I. Bengmark S. Acute portal vein stenosis. An experimental study on portal circulation and hepatosplenic function. Acta Chirurgica Scandinavica. 1985; 151:125-131

Rubin R, Haskal Z, O'Brien C, et.al Transjugular intrahepatic portosystemic shunting. Decreased survival for patients with high APACHE II scores. Am J Gastroenterol 1995;90:563 
Rueff B, Prandi D, Sicot J, Degos JD, Sicot C, Maillard JN, Fauvert R et al. A controlled study of therapeutic portacaval shunt in alcoholic cirrhosis. Lancet 1976;2:655-659.

Russo A, Giannone G, Magnano A, Passanisi G, Longo C. Prophylactic sclerotherapy in nonalcoholic liver cirrhosis: Preliminary results of a prospective controlled randomized trial. World Journal of Surgery 1989; 13:149-153.

Saari A, Klvilaakso E, Inberg M, Paakkonen M, Lahtinem J, Hockerstedt K, Schroder T. Comparison of somatostatin and vasopressin in bleeding esophageal varices. Am J Gastroenterol 1990;85:804-807.

Sacerdoti, D., Bolognesi, M., Merkel, C., Angeli, P., Gatta, A. Renal vasoconstriction in cirrhosis evaluated by Doppler ultrasonography. Hepatology 1993;17:219-24

Saggioro A, Pallini P, Vitalba A; Bortoluzzi F, Chiozzini G, Di Gilio A, et al. Complications of the endoscopic sclerotherapy of oesophageal varices: Comparison between different techniques. Policlinico - Sezione Chirurgica 1987; 94:461-464.

Santangelo WC, Dueno MI, Estes BL, Krejs GJ. Prophylactic sclerotherapy of large esophageal varices. New England Journal of Medicine 1988; 318:814818.

Sanyal A J, Freedman A M, Luketic V A, Purdum P P, Shiffman M L, Tisnado $\mathrm{J}$, Cole P E. Transjugular intrahepatic portosystemic stent shunts for patients with active variceal hemorrhage unresponsive to sclerotherapy. Gastroenterology 1996;111:138-146.

Sanyal AJ, Freedman AM, Luketic VA, Purdum III PP, Shiffman ML, Cole PE, Tisnado J, Simmons S Transjugular intrahepatic portosystemic shunts compared with endoscopic sclerotherapy for the prevention of recurrent variceal hemorrhage: $A$ randomized, controlled trial Annals of Internal Medicine, 1997;126:849-857

Sanyal AJ, Freedman AM, Shiffman ML, Purdum III PP, Luketic VA, Cheatham AK Portosystemic encephalopathy after transjugular intrahepatic portosystemic shunt: Results of a prospective controlled study. Hepatology 1994; 20:46-55

Sanyal, A.J., Freedman, A.M., Shiffman, M.L., Purdum III, P.P., Luketic, V.A., Cheatham, A.K.Portosystemic encephalopathy after transjugular intrahepatic portosystemic shunt: Results of a prospective controlled study. Hepatology $1994 ; 20: 46-55$

Sarin SK, Guptan RKC, Jain AK, Sundaram KR. A randomized controlled trial of endoscopic variceal band ligation for primary prophylaxis of variceal 
bleeding. European Journal of Gastroenterology and Hepatology 1996; 8:337-342.

Sauer P, Theilmann L, Roeren T, Richter G, Stremmel W, Sttiehl A. Transjugular intrahepatic portosystemic stent shunt (TIPS) vs Sclerotherapy in the prevention of variceal rebleeding : A randomised study. Gastroenterology 1996;110:A1313 (abstract)

Sauerbruch T, Wotzka R, Kopcke W, Harlin M, Heldwein W, Bayerdorfer E, et al. Prophylactic sclerotherapy before the first episode of variceal hemorrhage in patients wih cirrhosis. New England Journal of Medicine 1988; 319:8-15.

Schedl HP, Bartter FC An explanation for and experimental correction of the abnormal water diuresis in cirrhosis. J Clin Invest 1960;39:248-261

Schrier R W, Arroyo V, Bernardi M, Epstein M, Henriksen J H, Rodes J. Peripheral arterial vasodilatation hypothesis: a proposal for the initiation of renal sodium and water retention in cirrhosis. Hepatology, 1988;8: 11511157.

Sheen IS, Chen TY, Liaw YF. Randomized controlled study of propranolol for the prevention of recurrent esophageal varices bleeding in patients with cirrhosis. Liver 1989;9:1-5.

Sieber CC, Groszmann R J. Nitric oxide mediates hyporeactivity to vasopressors in mesenteric vessels of portal hypertensive rats. Gastroenterology, 1992;103: 235-239.

Sieber CC, Lopez-Talavera J C, Groszmann R J. Role of nitric oxide in the in vitro splanchnic vascular hyporeactivity in ascitic cirrhotic rats. Gastroenterology, 1993;104:1750-1754.

Silva G, Fluxa F, Bresky G, Backhouse C, Palma M, Ruiz M, Hirsch S, Iturriaga $\mathrm{H}$. Splanchnic and systemic haemodynamics in early abstinence and after ethanol administration in non-cirrhotic alcoholic patients. J Hepatol $1994 ; 20: 494-499$

Silvain C, Carpentier S, Sautereau D, Czernichow B, Metreau JM, Fort E, Ingrand $P$ et al. Terlipressin plus transdermal nitroglycerin vs octreotide in the control of acute bleeding from esophageal varices: a multicenter randomized trial. Hepatology 1993;18:61-65.

Simmons F, Goldstein H and Boyle J. A controlled clinical trial of lactulose in hepatic encephalopathy. Gastroenterol. 1970; 59: 827-832. 
Siringo S, McCormick PA, Mistry P, Kaye G, Mclntyre N, Burroughs AK. Prognostic significance of the white nipple sign in variceal bleeding. Gastrointestinal Endoscopy 1991; 37:51-55.

Smith JL, Graham DY The course of patients after variceal haemorrhage. Gastroenterology 1981;80:800-809

Soderlund C, Ihre T. Endoscopic sclerotherapy versus conservative management of bleeding oesophageal varices: a 5 year prospective controlled trial of emergency and long-term treatment. Acta Chir Scand. 1985; 151:449-456.

Soderlund C, Magnusson I, Torngren S, Lundell L. Terlipressin (triglycyllysine vasopressin) controls acute bleeding oesophageal varices. A doubleblind, randomized, placebo-controlled trial. Scand J Gastroenterol 1990;25:622-630.

Soehendra N, Grimm H, Nam V, et al. N-Butyl-2-cyanoacrylate: a supplement to endoscopic sclerotherapy. Endoscopy. 1987;19:221-224.

Soeters PB, Weir JC, Ebeid AM, Fischer JE Insulin, Glucagon, portasystemic shunting and hepatic failure in dogs. J Surg Res 1977;23:183188

Sogni $P$, Moreau R, Ohsuga M, Cailmail S et al. Evidence for normal nitric oxide-mediated vasodilator tone in conscious rats with cirrhosis. Hepatology, 1992;16: $980-983$

Solis-Herruzo J A, Duran A, Favela V et al. Effects of lumbar sympathetic block on kidney function in cirrhotic patients with hepatorenal syndrome. Journal of Hepatology, 1987;5: 167-173.

Soupison T, Yang S, Bernard C, Moreau R et al. Short-term hemodynamic responses to inhibition of tumor necrosis factor alpha by pentoxifylline in rats with cirrhosis. Journal of Hepatology, 1994;21 (suppl 1): S45.

Spahr L, Butterworth RF, Fontaine S, Bui L, Therrien G, Milette PC, Lebrun $\mathrm{LH}$, Zayed J, Leblanc A, PomierLayrargeus $\mathrm{G}$ Increased blood manganese in cirrhotic patients: Relationship to pallidal magnetic resonance signal hyperintensity and neurological symptoms. Hepatology 1996; 24: 1116-1120

Spina GP, Santambrogio R, Opocher E, Cosentino F, Zambelli A, Rubis Passoni G, et al. Distal splenorenal shunt versus endoscopic sclerotherapy in the prevention of variceal rebleeding. First stage of a randomized, controlled trial. Annals of Surgery 1990; 211:178-186. 
Stahl G E. (1748) De venae portae: In Halter: Disputationes anatomical, 3: 31. Cited by Child. 1954.

Stahl, J. Studies of the blood ammonia in liver disease. Its diagnostic, prognostic and therapeutic significance. Ann Int Med. 1963;58: 1-24.

Stanley A J, Jalan R, Ireland H M, Redhead D N, Bouchier I A D, Hayes P C. A comparison between gastric and oesophageal variceal haemorrhage treated with transjugular intrahepatic portosystemic stent shunt (TIPSS). Alimentary Pharmacology \& Therapeutics 1997;11:171-176.

Steigmann G, Goff GS. Endoscopic esophageal varix ligation: Preliminary clinical experience. Gastrointestinal Endoscopy 1988;34:113-117

Steigmann, G.V., Goff, J.S., Michaletz-Onody, P.A., Korula J., Lieberman D, Saeed ZA, Reveille RM, Sun JH, Lowenstein SR. Endoscopic sclerotherapy as compared with endoscopic ligation for bleeding esophageal varices. $\mathrm{N}$ Eng J Med. 1992; 326: 1527-1532

Stipa S, Ziparo V, Anza M. A randomized controlled trial of mesenteriocaval shunt with autologous jugular vein. Surg Gynecol Obstet. 1981;153:353356.

Strauss E, de Sa MFG, Albano A, Lacet CMC, Leite MO, Maffei RA Jr. A randomised controlled trial for the prevention of the first upper gastrointestinal bleeding due to portal hypertension in cirrhosis: sclerotherapy or propranolol versus control groups (Abstract). Hepatology 1988;8:1395.

Sung JJ, Chung SCS, Lai CW, Chan FKL, Leung JWC, Young MY. Octreotide infusion of emergency sclerotherapy for variceal hemorrhage. Lancet 1993;342:637-641.

Sushma S, Dasarathy S, Tandon RK, Jain S, Gupta S and Bhist MS. Sodium benzoate in the treatment of acute hepatic encephalopathy: a double-blind randomised trial. Hepatology. 1992; 16: 138-144.

Tage-Jensen U. Henriksen JH. Christensen E. Widding A. Ring-Larsen H. Christensen NJ. Plasma catecholamine level and portal venous pressure as guides to prognosis in patients with cirrhosis. Journal of Hepatology. 1988;6:350-358

Terblanche J, Bornman PC, Kahn D, Jonker MA, Campbell JAH, Kirsch R. Failure of repeated injection sclerotherapy to improve long term survival after oesophageal variceal bleeding. A five-year prospective controlled clinical trial. Lancet 1983;2:1328-1332. 
Teres J, Baroni R, Bordas JM, Vias J, Pera C, Rodes J. Randomized trial of portacaval shunt, stapling transection and endoscopic sclerotherapy in uncontrolled variceal bleeding. J Hepatol 1987B;4:159-167.

Teres J, Bordas JM, Bravo D, Visa J, Grande L, Garcia-Valdecanas JC, Pera $\mathrm{C}$ et al. Sclerotherapy vs distal splenorenal shunt in the elective treatment of variceal haemorrhage: a randomized controlled trial. Hepatology 1987A;7:430-436.

Teres J, Bosch J, Garcia Pagan JC, Feu F, Cirera I, Rodes J. Propranolol vs sclerotherapy in the prevention of variceal rebleeding: a randomised controlled trial. Gastroenterology 1993;105:1508-1514.

The Copenhagen Esophageal Varices Sclerotherapy Project. Sclerotherapy after first variceal hemorrhage in cirrhosis. A randomized multicenter trial. $\mathrm{N}$ Engl J Med 1984;311:1594-1600.

The PROVA Study Group. Prophylaxis of first hemorrhage from oesophageal varices by sclerotherapy, propranolol or both in cirrhotic patients. A randomised multicenter trial. Hepatology 1991;14:1016-1024.

The Veterans Affairs Cooperative Variceal Sclerotherapy Group. Prophylactic sclerotherapy for esophageal varices in alcoholic liver disease: a randomised, single-blind, multicenter clinical trial. $N$ Engl J Med 1991;324:1779-1784.

Thomas P G, D'Cruz A J. Distal splenorenal shunting for bleeding gastric varices. British Journal of Surgery 1994;81:241-244.

Tobe SW, Blendis LM, Morali GA, Warner LC, Logan AG, Skorecki KL. Angiotensin II modulates atrial natriuretic factor-induced natriuresis in cirrhosis with ascites. American Journal of Kidney Diseases 1993;21:472479

Trew M, Maguire P.Further comparison of two instruments for measuring quality of life in cancer patients. In: Quality of life. Beckman. J., (ed.) 1984; pg. 111. Proc. Third Workshop of the EORTC study group on Quality of Life. Paris.

Triger DR, Smart HL, Hosking SW, Johnson AG. Prophylactic sclerotherapy for esophageal varices: Long-term results of a single-center trial. Hepatology 1991; 13:117-123.

Tsai YT, Lay CS, Lai KH, Ng WW, Yeh YS, Wang JY, Chiang TT et al. Controlled trial of vasopressin plus nitroglycerin vs vasopressin alone in the treatment of bleeding esophageal varices. Hepatology 1986;6:406-409. 
Tygstrup N, Winkler K, Mellemgaard K Andreassen M. Determination of the hepatic arterial blood flow and oxygen supply in man by clamping the hepatic artery during surgery. Journal of Clinical Investigation, 1962;41: 447-454.

Uchihara M, Izumi N, Sato C, Marumo F. Clinical significance of elevated plasma endothelin concentration in patients with cirrhosis. Hepatology, 1992;16: 95-99.

VA Cooperative Variceal Sclerotherapy Group. Sclerotherapy for male alcoholic cirrhotic patients who have bled for esophageal varices: results of a randomized multicenter clinical trial. Hepatology 1994;20:618-625.

Valenzuela JE, Schubert T, Fogel MR, Strong RM, Levine J, Mills PR, et al. A multicenter, randomized, double-blind trial of somatostatin in the management of acute hemorrhage from esophageal varices. Hepatology $1989 ; 10: 958-961$.

Valla D., Bercoff E., Menu Y., et.al.Discrepancy between wedged hepatic venous pressure and portal venous pressure after acute propranolol administration in patients with alcoholic cirrhosis. Gastroenterology. 1984; 86:1400-03

Vallance $\mathrm{P}$, Moncada S. Hyperdynamic circulation in cirrhosis: a role for nitric oxide? Lancet, 1991;337: 776-778.

Vanhoutte P M. Vascular effects of serotonin and ischaemia. Journal of Cardiovascular Pharmacology, 1990;16 (suppl 3): S15-S19.

Variceal Bleeding Study Group. Hospitals Cinic (Barcelona), Germans Trias (Badalona), Gregorio Maranon (Madrid), Ramon y Cajal (Madrid). Double blind comparison of somatostatin infusion vs glypressin injection in the treatment of acute variceal hemorrhage in patients with cirrhosis. (Abstract). J Hepatol 1993;18(suppl 1):S37.

Vianna A, Hayes P, Moscoso $G$ et al. Normal venous circulation of the gastroesophageal junction. $A$ route to understanding varices. Gastroenterology, 1987;93: 876-889.

Villeneuve JP, Pomier-Layrargeus G, Infante-Rivard C, Willems B, Huet PM, Marleau D, Viallet $A$ Propranolol for the prevention of recurrent variceal haemorrhage: a controlled trial. Hepatology 1986;6:1239-1243

Vinel JP, Cassingeul J, Levade $M$ et al. Assessment of short term prognosis after variceal bleeding in patients with alcoholic cirrhosis by early measurement of porto-hepatic gradient. Hepatology 1986;6:116-117. 
Vorobioff J, Garcia-Tsao G, Groszmann R, Aceves G et al. Longterm haemodynamic effects of ketanserin, a 5-hydroxytryptamine blocker, in portal hypertensive patients. Hepatology, 1989;9: 85-91.

Walker S, Kreichgauer HP, Bode JC. Terlipressin vs somatostatin in bleeding esophageal varices: a controlled double blind study. Hepatology 1992; 15:1023-1030.

Westaby D, Polson RJ, Gimson AES, Hayes PC, Hayllar K, Williams R. A controlled trial of oral propranolol compared with injection sclerotherapy for the long-term management of variceal bleeding. Hepatology 1990;11:353359.

Westaby D, Hayes P, Gimson A, et al. Controlled trial of injection sclerotherapy for active variceal bleeding. Hepatology 1989;9:274-277.

Westaby D, MacDougall BRD, Williams R. Improved survival following injection sclerotherapy for oesophageal varices: final analysis of a controlled trial. Hepatology. 1985;5:627-631.

Willet I R, Esler M, Jennings $G$ and Dudley F J. Sympathetic tone modulates portal venous pressure in alcoholic cirrhosis. Lancet, 1986;1: 939-942.

Williams S G J, Peters R A, Westaby D. Thrombin - an effective treatment for gastric variceal haemorrhage. Gut 1994;35:1287-1289.

Wilson, B., Cockburn, J., Baddeley, A., Hlorns, R. The development and validation of a test battery for detecting and monitoring everyday memory problems. J.Clin.Exp. Neuropsychol. 1989; 6: 855-870

Witte $\mathrm{C} L$ and Witte $\mathrm{M} \mathrm{H}$. Splanchnic circulatory and tissue fluid dynamics in portal hypertension. Federation Proceedings, 1983;42: 1685-1689.

Witte $\mathrm{Cl}$, Witte $\mathrm{MH}$, Dumont A E. The portal triad in hepatic cirrhosis. Surgery, Gynae, ... 1978; 146, 965-974.

Witte $\mathrm{M} \mathrm{H}$, Witte $\mathrm{C} L$, Dumont A E. Progress in liver disease: physiological factors involved in the causation of cirrhotic ascites. Gastroenterology 1971;61: 742-750.

Witzel L, Wolbergs E, Merki H. Prophylactic endoscopic sclerotherapy of oesophageal varices. A prospective controlled study. Lancet 1985; 1:773775 .

Wong F, Gigrah N, Blendis L. Review: The controversy over the pathophysiology of ascites formation in cirrhosis. Journal of Gastroenterology and Hepatology 1997;12:437-444 
Wong F, Massie D, Hsu P, Dudley F. Renal response to a saline load in wellcompensated alcoholic cirrhosis. Hepatology, 1994;20: 873-881.

Wong, F, Massie, D., Hsu, P., Dudley, F. Renal response to a saline load in well compensated alcoholic cirrhosis. Hepatology. 1994;20:873-81

Wong, F., Sniderman, K., Liu, P., Blendis, L. Dissecting the factors involved in the pathogenesis of sodium retention in cirrhosis with ascites using TIPS. Hepatology. 1995;22: 164A (abstract).

Wordehoff D, Spech HJ. Prophylactic sclerotherapy of esophageal varices: Results of a prospective, randomized long-term trial over seven years. Deutsche Medizinische Wochenschrift 1987; 112:947-951.

Wu Y, Burns R C, Sitzmann J V. Effects of nitric oxide and cyclooxygenase inhibition on splanchnic haemodynamics in portal hypertension. Hepatology, 1993;18: 1416-1421

Wu Y, Li S S, Campbell K A, Sitzmann J V. Modulation of splanchnic vascular sensitivity to angiotensin II. Surgery, 1991;110: 162-168.

Zigmond, A.S., Snaith, R.P.The Hospital Anxiety and Depression Scale.Acta.Psychiatrica. Scandinavica. 1983; 67: 361-370.25. 
1. Transjugular intrahepatic portosystemic stent shunt (TIPSS): long term follow-up. Jalan R, Simpson KJ, Redhead DN, Chalmers N, Hayes PC Quarterly Journal of Medicine1994;87:565-73

2. Wall or Palmaz stents for TIPSS Jalan, R., Ferguson, J., Redhead, D.N., Elton, R.A., Hayes, P.C. Journal of Interventional Radiology 1994;9:147-52

3. The role of Duplex Doppler in monitoring shunt function following transjugular intrahepatic stent shunt. Ferguson J, Jalan R, Redhead DN, Hayes PC, Allan PA British J Radiology. 1995;68:587-589

4. Analysis of prognostic variables in the prediction of shunt failure, variceal rebleeding, early mortality and encephalopathy following the Transjugular intrahepatic portosystemic stent-shunt (TIPSS) Jalan R, Elton RA, Redhead, DN, Simpson KJ, Finlayson NDC, Hayes PC. J Hepatology 1995;23:123-128. 
5. Transjugular intrahepatic portosystemic stentshunt (TIPSS) occlusion and the role of biliaryvenous fistula. Jalan R, Harrison DJ, Redhead DN, Hayes PC. Journal of Hepatology 1996;24:169-76

6. A comparative study of the transjugular intrahepatic portosystemic stent-shunt (TIPSS) and oesophageal transection in uncontrolled variceal haemorrhage. Jalan R, John,T.G., Redhead DN, Finlayson, NDC, Garden OJ, Hayes PC. Am J Gastroenterol 1995;90:1932.

7. Haematological alterations following the transjugular intrahepatic portosystemic stent-shunt (TIPSS). Jalan R, Redhead DN, Hayes PC. Eur J Gastroenterol \& Hepatol 1996;8:381-385.

8. Direct measurement of portal pressure and variceal haemorrhage. Jalan R, Redhead DN, Forrest EH, Hayes PC American Journal of Gastroenterology 1993;88:2140. 
9. A prospective study of changes in neuropsychological and liver function following the transjugular intrahepatic portosystemic stent shunt. Jalan R, Gooday R, Carrol R, Redhead DN, Simpson KJ, Hayes PC. J Hepatology 1995;23:123-128.

10. Intentional reversible thrombosis of TIPSS and renal blood flow, Devil's advocate Jalan $R$, Hayes PC. Radiology 1995;197:587-588

11. Differing actions of propranolol and nitrates in cirrhosis: A rationale for combination therapy to prevent variceal haemorrhage? Forrest EH, Jalan R, Redhead DN, Hayes PC. Alimentary Pharmacology and Therapeutics 1996;10:795-800

12. Reduction in renal blood flow following acute increase in the portal pressure: Evidence for the existence of a hepatorenal reflex in man? Jalan $R$, Forrest EH, Redhead DN, Dillon JF, Hayes PC Gut, 1997;40:664-670

13. Mechanism of changes in renal function following TIPSS Jalan R, Thomas HW, Henderson N, O'Rourke 
K, Dillon JF, Williams BC, Hayes PC European J Gastroenterology and Hepatology 1996;8:1111-1116

14.Changes in Plasma Biogenic Amines after Transjugular Intrahepatic Portosystemic Stent-Shunt and Their Potential Role in Hepatic Encephalopathy. R Jalan, YL Chung, K Bhakoo, Peter C Hayes, JD Bell Recent advances in the pathogenesis of ammonia metabolism. (in press)

15. A comparison of gastric and oesophageal varices treated with TIPSS. Stanley AJ, Jalan R, Ireland IM, Redhead DN, Hayes PC Alimentary Pharmacology and Therapeutics (in press)

16. TIPSS as a bridge to OLT John, TG, Jalan, R, Stanley AJ, Redhead, DN, Sanfey, H, Hayes, PC, Garden, OJ European J Gastroenterology and Hepatology 1996;8:1145-1149

17. Transjugular intrahepatic portosystemic stentshunt: Results in 130 patients. Stanley AJ, Jalan R, Redhead DN, Finlayson NDC, Hayes PC. Gut 1996;39:479-485 
18. TIPSS vs variceal band ligation in the secondary prevention of variceal haemorrhage in cirrhosis: Results of a randomised controlled study. Jalan, R., Forrest, E.H., Redhead, D.N., Dillon, J.F., Finlayson, N.D.C., MacGilchrist, A.J., Hayes, P.C. Hepatology 1997;26:1115-1122

19. Elevation in intracranial pressure after TIPSS. Jalan R, Dabos K, Redhead DN, A Lee, Hayes PC J Hepatol 1997;27:928-933)

20. Transjugular intrahepatic portosystemic stentshunt (TIPSS): A Review Jalan, R., Redhead, D.N., Hayes, P.C. National Medical Journal of India. 1995;8:15-21

21. A Review of Transjugular intrahepatic portosystemic stent-shunt (TIPSS) for variceal haemorrhage Jalan, R., Redhead, D.N., Hayes, P.C. Br J Surgery 1995;82:1158-1164

22. Shunt insufficiency after TIPSS: The whats, whys, hows and what should we do about it? Jalan $R$, 
Stanley AJ, Redhead DN, Hayes PC Clinical Radiology (Editorial) 1997;52:329-331

23. Seminars in Portal Hypertension, Pathogenesis and Management of Ascites and Chronic Hepatic Encephalopathy. Jalan R, PC Hayes Lancet 1997;350:1309-1315

24. TIPSS trials: Design determines outcome. Jalan R, NDC Finlayson, PC Hayes Hepatology 1997;26:1361-1365 